University of Louisville ThinkIR: The University of Louisville's Institutional Repository

Electronic Theses and Dissertations

$12-2015$

\title{
Development of a vascularized, induced pluripotent stem cell-derived liver-tissue mimic for therapeutic applications.
}

Venkat Madhav Ramakrishnan

University of Louisville

Follow this and additional works at: https://ir.library.louisville.edu/etd

Part of the Physiological Processes Commons, and the Systems and Integrative Engineering Commons

\section{Recommended Citation}

Ramakrishnan, Venkat Madhav, "Development of a vascularized, induced pluripotent stem cell-derived liver-tissue mimic for therapeutic applications." (2015). Electronic Theses and Dissertations. Paper 2319.

https://doi.org/10.18297/etd/2319

This Doctoral Dissertation is brought to you for free and open access by ThinkIR: The University of Louisville's Institutional Repository. It has been accepted for inclusion in Electronic Theses and Dissertations by an authorized administrator of ThinkIR: The University of Louisville's Institutional Repository. This title appears here courtesy of the author, who has retained all other copyrights. For more information, please contact thinkir@louisville.edu. 


\title{
DEVELOPMENT OF A VASCULARIZED, INDUCED PLURIPOTENT STEM CELL- DERIVED LIVER-TISSUE MIMIC FOR THERAPEUTIC APPLICATIONS
}

\author{
by
}

Venkat Madhav Ramakrishnan

B.S., University of Louisville, 2010

M.S., University of Louisville, 2014

\author{
A Dissertation \\ Submitted to the Faculty of the \\ School of Medicine of the University of Louisville \\ In Partial Fulfillment of the Requirements \\ For the Degree of
}

Doctor of Philosophy in Physiology and Biophysics

Department of Physiology

University of Louisville

Louisville, KY

December 2015 
(C) 2015 by Venkat Madhav Ramakrishnan

All rights reserved 



\title{
DEVELOPMENT OF A VASCULARIZED, INDUCED PLURIPOTENT STEM CELL- DERIVED LIVER-TISSUE MIMIC FOR THERAPEUTIC APPLICATIONS
}

\author{
by
}

Venkat Madhav Ramakrishnan

B.S., University of Louisville, 2010

M.S., University of Louisville, 2014

A Dissertation Approved on

November 23, 2015

by the following Dissertation Committee:

\begin{tabular}{c}
\hline Nolan L. Boyd, Ph.D., Dissertation Director \\
\hline Gavin E. Arteel, Ph.D. \\
\hline Patrick D. Harris, Ph.D. \\
\hline James B. Hoying, Ph.D. \\
\hline Irving G. Joshua, Ph.D. \\
\hline
\end{tabular}

Stuart K. Williams, II, Ph.D. 


\section{DEDICATION}

This dissertation is dedicated to my paternal grandfather, M.P. Govind; paternal grandmother, A. Sarojini Govind; paternal aunt, Vimala Pakath; and maternal grandmother, A. Sarojini Madhavan, all of whom sadly passed away during my medical and graduate training. I also dedicate this dissertation to my parents, Dr. Ramakrishnan Pakath and Sreedevi Ramakrishnan, for their sacrifices, love, encouragement, and support. 


\section{ACKNOWLEDGEMENTS}

First and foremost, I thank my graduate advisor, Dr. Nolan Boyd, for his guidance, instruction, mentorship, patience, and for continually pushing me to do my best. I also thank my committee members, Dr. Gavin Arteel, Dr. Patrick Harris, Dr. James Hoying, Dr. Irving Joshua, and Dr. Stuart Williams for their comments, support, and assistance over the past three years. I sincerely thank Dr. Franklin West and Dr. Jeong-Yeh Yang for their expert assistance with our stem cell lines; Dr. Richard Wade-Martins for his guidance and expertise with his lab's non-integrating, non-viral, episomal plasmid; Patrick Burchell, Dr. Sara Nunes, Dr. John Maijub, and Dr. Marvin Morris, for their assistance and support; Dr. Terry McCurry, Dr. Scott Whittemore, Dr. Michal Hetman, and Dr. Lukasz Slomnicki for their experimental provisions; Jacob Dale, Robert Reed, and Jason Beare for their technical assistance; and Mary Boyd, for her editorial assistance. I thank my student trainees, Kevin Tien, Braden Bocard, and Thomas McKinley, for their hard work and dedication to the lab. I also extend my heartfelt thanks to Dr. Donald Miller, Dr. Binks Wattenberg, and Victoria King of UofL's combined MD-PhD program for their guidance and for supporting my dream of becoming a physician-scientist. Last but not least, I could not have done any of this without the support, advice, and understanding of my family and friends. 


\begin{abstract}
DEVELOPMENT OF A VASCULARIZED, INDUCED PLURIPOTENT STEM CELLDERIVED LIVER-TISSUE MIMIC FOR THERAPEUTIC APPLICATIONS

Venkat Madhav Ramakrishnan
\end{abstract}

November 23, 2015

This dissertation describes the incorporation of several technologies (stem cells, gene therapy, tissue engineering and regenerative medicine) into a single project that aims to produce a liver-tissue mimic for therapeutic applications. The liver is arguably one of the most complex organs in the body. In addition to its remarkable capacity to regenerate, it performs a host of vital functions. As a result, its impairment has widespread systemic consequences. The work described herein focused on the liver in the context of cardiovascular disease and used the heritable disorder Familial Hypercholesterolemia (FH) as a clinical disease model. As (a) the only definitive cure for FH is currently liver transplant and (b) the availability of quality liver organs for transplant is critically low, these studies seek to develop a liver-tissue mimic comprised of two parts: functional hepatocyte-like cells (derived from induced pluripotent stem cells, or iPSC) and vascular support (provided by adipose-derived stromal vascular fraction (SVF)).

The dissertation is divided into six sections. Chapter I provides an introductory 
overview and lists the aims and hypotheses for the dissertation. Chapter II provides a four-part background discussing cholesterol metabolism, the liver organ, stem cells, and the vasculature. Chapter III describes our efforts to generate a proof-of-concept livertissue mimic, using HepG2 as a hepatocyte model cell source and SVF cells as the vascular support system. As vascular support is critical for parenchymal survival and function, Chapter IV examines the mechanisms of spontaneous SVF vascular selfassembly. Chapter V discusses development of a patient-specific, therapeutic cell system. FH-patient dermal fibroblasts were programmed into iPSC using modRNA technology, and eventually subjected the iPSC to directed differentiation into hepatocyte-like cells ${ }^{1}$. Yet, as the iPSC were derived from an $\mathrm{FH}$ patient, the cells required functional restoration of their LDL-R in order to impart any therapeutic benefit. To accomplish this, a novel episomal LDL-R plasmid containing (a) upstream regulatory control sequences that confer physiological feedback control of LDL-R expression and (b) Epstein-Barr sequences for episomal retention and replication was used. To mitigate any potential concerns associated with viral vectors, the iPSC were derived and corrected using nonviral modalities. These cells were combined with an SVF derived vascular support system to assess iPSC-HLC survival characteristics in vivo. Chapter VI provides a comprehensive discussion regarding our experimental efforts.

These experiments demonstrate the development of a vascularized, iPSCderived hepatocyte liver-tissue mimic that could potentially be used for therapeutic applications, such as for the treatment of FH. Efforts to create this tissue engineered liver construct were guided by three aims: (1) Assess the role of adipose SVF in providing vascular support to implanted parenchymal cells, (2) Evaluate and define the 
mechanism(s) of SVF vascular self-assembly, and (3) Restore the functionality of monogenic-deficient FH cells. These studies provide several proofs-of-principle towards the development of effective cell-based treatments, not only for $\mathrm{FH}$, but also for other diseases classically requiring whole-organ transplantation. 


\section{TABLE OF CONTENTS}

ACKNOWLEDGEMENTS

iv

ABSTRACT

$\mathrm{V}$

LIST OF TABLES

$\mathrm{x}$

LIST OF FIGURES

$\mathrm{xi}$

CHAPTER I: DISSERTATION INTRODUCTION \& SPECIFIC AIMS

CHAPTER II: BACKGROUND / LITERATURE REVIEW

PART I: Cholesterol and Disease

PART II: The Liver and Hepatic Substitutes

PART III: Stem Cells

PART IV: The Vasculature

CHAPTER III: Generation of a Functional Liver-Tissue Mimic Using Adipose

Stromal Vascular Fraction Cell-Derived Vasculatures

OVERVIEW

INTRODUCTION

MATERIALS \& METHODS

RESULTS

DISCUSSION

CHAPTER IV: Wnt5a Modulates the Assembly of Human Stromal Vascular

Fraction-Derived Microvasculatures

OVERVIEW 
$\begin{array}{ll}\text { INTRODUCTION } & 85\end{array}$

MATERIALS \& METHODS $\quad 87$

$\begin{array}{ll}\text { RESULTS } & 97\end{array}$

$\begin{array}{lr}\text { DISCUSSION } & 116\end{array}$

CHAPTER V: Restoration of Physiologically Responsive Low-Density

Lipoprotein Receptor-Mediated Endocytosis in Genetically-Deficient Induced

Pluripotent Stem Cells

OVERVIEW

INTRODUCTION

MATERIALS \& METHODS

RESULTS

DISCUSSION

CHAPTER VI: SUMMARY AND CONCLUSIONS

REFERENCES 


\section{LIST OF TABLES}

TABLE PAGE

Table 1: Chapter II: Common Angiogenic Inhibitors 89

Table 2: Chapter II: RT-PCR primer listing $\quad 91$

Table 3: Chapter II: Antibody / Stain / Lectin listing 93

$\begin{array}{ll}\text { Table 4: Chapter III: Electroporation parameters } & 128\end{array}$

Table 5: Chapter III: RT-PCR primer listing 134

Table 6: Chapter III: Antibody / Stain listing 136 


\section{LIST OF FIGURES}

FIGURE

Figure 1: Mechanism of cholesterol synthesis from Acetyl-CoA 8

Figure 2: LDL-R Feedback Loop 20

Figure 3: Episomal LDL-R plasmid 26

Figure 4: Liver microscopic anatomy 32

Figure 5: Liver sinusoids 32

Figure 6: Fresh vs. cultured SVF in vivo 70

Figure 7: Fresh vs. cultured SVF \& angiogenic participation 72

Figure 8: FACS analysis on fresh vs. cultured SVF 74

Figure 9: hSVF in vivo \& with HepG2 spheroids 75

Figure 10: SVF within a liver-tissue mimic facilitates LDL uptake $\quad 78$

$\begin{array}{ll}\text { Figure 11: hSVF angiogenic inhibition } & 90\end{array}$

Figure 12: EC network tracing and area determination 96

Figure 13: Culture reduces hSVF vascular potential 98

Figure 14: Wnt5a mediates hSVF vascular assembly 100

Figure 15: Wnt \& Wnt5a receptor PCR screening 102

Figure 16: Wnt5a \& FZD4 are highly expressed in hSVF cultures 103-104

Figure 17: rhWnt5a mediates hSVF EC assembly during IWP2 inhibition 106-107

Figure 18: IWP-treated hSVF EC networks are not affected by rhWnt3a $\quad 109-110$ 
Figure 19: Anti-Wnt5a blocks hSVF EC length and segments 112

Figure 20: Wnt5a drives EC microvascular assembly in vivo 113-114

Figure 21: FH fibroblast LDL-R functional assessment 138

Figure 22: FH-iPSC derivation and pluripotency 140

Figure 23: FH-iPSC karyotyping 142

Figure 24: FH-HLC derivation 144

Figure 25: Stage-specific gene expression during FH-HLC derivation $\quad 146$

Figure 26: FH-HLC functional assessment 147

Figure 27: $p E H Z-L D L R-L D L R$ functionality in CHO-a7 149

Figure 28: Functionality of FH-HLC \pm LDLR $\quad$ 153-154

Figure 29: Quantitation of plasmid functionality in FH-MC \pm LDLR 156

Figure 30: Concept of Wnt5a and hSVF self-assembly 171 


\section{CHAPTER I}

\section{DISSERTATION INTRODUCTION \& SPECIFIC AIMS}

Multiple genetic diseases of the liver, including fulminant hepatic failure, nonalcoholic fatty liver disease (NAFLD), alpha-1 antitrypsin (A1AT) deficiency, and familial hypercholesterolemia $(\mathrm{FH})$, are only functionally cured by orthotopic liver transplant. Yet, existing deficiencies in transplant teleology (the requirement for immunosuppression, the risk of rejection, and consequent loss of quality of life) have launched efforts to regenerate cells and tissues to mitigate such dysfunction.

Induced pluripotent stem cells (iPSC) are a novel, theoretically limitless source of cells that could potentially address these issues and others, as they can differentiate down any cell lineage and be derived autologously, eliminating any need for the immunosuppression associated with transplant. However, iPSC from genetically impaired patients would differentiate into syngeneic replacement cells that are fully compatible with the host, but just as dysfunctional as targeted diseased tissue. Therefore a critical need in advancing iPSC as a cell-based therapeutic is an appropriate strategy for correcting the clinical deficiency. Though genetic origin of disease often has multiple

sources, monogenic disorders could theoretically be corrected by the introduction of a single functional gene product. One example of monogenic disease is Familial Hypercholesterolemia (FH), characterized by dysfunction of the LDL-cholesterol 
receptor $(L D L R)$. FH has been extensively studied and represents a suitable model for investigating the functional correction of genetically dysfunctional iPSC, which can then be used to generate a liver tissue mimic.

Efforts to restore the functionality of primary and derived hepatocytes have resulted in varying degrees of success. $L D L R$-containing corrective plasmids have been delivered to hepatocytes via adeno-associated ${ }^{2}$ and retroviral vectors ${ }^{3}$. In numerous instances, the high transduction efficiencies afforded by viral vectors yielded significant reductions in plasma LDL cholesterol (LDL-C). However, concerns about transgene activation, insertional mutagenesis ${ }^{4}$, and oncogenesis ${ }^{5}$ (all of which can result in patient morbidity and/or mortality) hamper broad clinical use of such viral vectors for gene therapy. Non-viral transfection modalities represent a practical alternative, but these typically suffer from transient expression and do not provide long-term resolution. Our approach to address these issues is utilization of a novel corrective plasmid that was designed to be episomal and non-integrating, stably transfected, and physiologically sensitive $^{6}$. The latter feature is especially relevant, as corrective $L D L R$ plasmids lacking physiologic sensitivity could force cells to internalize LDL-C without regulation or inhibition, leading to toxic cholesterol saturation and eventual cell dysfunction ${ }^{7}$.

The generation of a supportive vasculature is also important, as it would serve as a vital conduit for parenchymal nutrient delivery and metabolic byproduct expulsion. The literature provides evidence of implanted parenchymal cell death in the absence of a vascular supply. Investigations have also demonstrated a need for co-cultured support cells that enhance parenchymal viability ${ }^{8}$. Such findings suggest that a stable, functional microvasculature is required for parenchymal cell survival and function. We have already 
demonstrated that adipose-derived stromal vascular fraction (SVF) is an easily accessible, heterogeneous mixture of cells capable of providing stromal and vascular support to implanted parenchymal hepatocytes ${ }^{9}$. In our hands, SVF cells formed a functional microcirculation that spontaneously assembled, interfaced with these hepatocytes, and anastomosed with surrounding host vasculature. Hepatocytes were able to internalize peripherally injected circulating LDL-C, providing further evidence for the formation of a functional, SVF-derived vasculature at the host-parenchyma interface. The nuclei of cells cultured without SVF exhibited characteristics of apoptosis, whereas those cultured with SVF appeared intact and ostensibly healthy. This combination of hepatocytes with SVF cells formed the basis for a functional liver-tissue mimic.

In conclusion, we aim to use a monogenic disease model for exploring the nonviral, functional restoration of genetically dysfunctional iPSC. Corrected iPSC will be used to engineer and generate hepatocyte-like cells that will be assessed for restored functionality as well as for evidence of maturation in the presence of an SVF cell coculture. The reciprocal effect of the parenchyma on the newly developed, surrounding microvasculature will also be assessed. The development of a functional tissue mimic lays the groundwork for further exploration into the architecture, size, and scalability of engineered organoids specific to various dysfunctional phenotypes. This translational project will contribute to the development of cell-based therapies and other autologous, transplantable organs.

Globally, I hypothesize that functionally corrected autologous FH-iPSCderived parenchymal cells, combined with stromal and vascular support, will form following implantation a functional tissue mimic that offers sustainable therapeutic 
value in end-organ dysfunction. This hypothesis will be examined via the following specific aims:

Aim 1: Assess the role of Stromal Vascular Fraction (SVF) cells in providing vascular support to implanted hepatocytes.

Aim 1 Hypothesis: An SVF-derived vasculature enables the engraftment and function of implanted hepatocytes.

Aim 1 Rationale: The hypothesis for this aim is predicated on SVF's documented ability to rapidly self-assemble into vessels. We further postulated that SVF could likely form self-assembled vasculatures in the presence of hepatocytes.

\section{Aim 2: Define mechanisms of human SVF vascular assembly.}

Aim 2 Hypothesis: Wnt5a mediates hSVF vascular assembly.

Aim 2 Rationale: Our preliminary data indicates that the pan Wnt-release inhibitor IWP2 inhibits endothelial cell (EC) networking in SVF, implicating all Wnt isoforms in EC network formation. The literature has suggested that Wnt5a is implicated in processes such as perivascular recruitment, vascular sprouting, vascular pruning, and tight-junction maintenance, all of which are intimately associated with EC.

\section{Aim 3: Restore the functionality of monogenic-deficient $\mathrm{FH}$ parenchymal cells.}

Aim 3 Hypothesis: A non-viral, non-integrating episomal plasmid containing a wild-type LDL receptor (LDL-R) cassette will restore LDL-R functionality to FHiPSC-derived hepatocytes. 
Aim 3 Rationale: The technology and function of an episomal LDL-R-containing plasmid has already been demonstrated in the literature. Additionally, iPSC have been shown to generate autologous hepatocyte-like cells (HLC) in addition to a variety of other parenchymal cell types. Further, iPSC can be easily cultured and are amenable to genetic manipulation. 
CHAPTER II

\section{BACKGROUND \& LITERATURE REVIEW}

\section{PART I: CHOLESTEROL \& DISEASE}

\section{Cholesterol Production}

The liver is the primary organ responsible for cholesterol regulation in the body ${ }^{10}$. Cholesterol is an important constituent of cell membranes and several cellular byproducts, including steroids, hormones, and bile ${ }^{11}$. Systemic cholesterol homeostasis is tightly regulated, between inputs (such as cholesterol obtained through cell synthesis or through the diet) and outputs (such as the byproducts described above). Therefore, a long-term increase in plasma cholesterol is a result of deregulated homeostasis leading to accelerated development of cardiovascular disease (e.g. atherosclerosis, cardiac infarction) and death ${ }^{12}$.

Before discussion of the implications of cholesterol in disease, it is important to outline the mechanisms of cholesterol synthesis and metabolism (Figure 1). The process of cholesterol synthesis begins with Acetyl CoA, a central intermediate in lipid metabolism $^{13}$. The breakdown of glucose or amino acids yields Acetyl CoA, whose downstream products include fatty acids and ketone bodies, triglycerides, phospholipids, eicosanoids, and cholesterols, as well as carbon dioxide, water, and ATP (energy) in 
cellular respiration. Specifically, with respect to cholesterol, Acetyl CoA is converted to 3-hydroxy-3-methylglutaryl-CoA (HMG-CoA) and then Mevalonate, whose generation is mediated by the enzyme HMG-CoA reductase (HMGCR). HMGCR serves as the ratelimiting step of cholesterol synthesis and a target for several pharmacological therapies (discussed below). Additionally, the ultimate conversion of Acetyl CoA to Mevalonate constitutes the first stage of cholesterol synthesis. The second stage converts Mevalonate into several 5-carbon building blocks, while the third stage generates lipid intermediates of biological importance. For instance, one of these intermediates, Farnesyl phosphate, is a branch point intermediate in ubiquinone and dolichol synthesis. Ubiquinone is an important electron carrier in the electron transport chain, and dolichol is an important endoplasmic reticulum anchor involved in N-linked glycosylation. Thus, these intermediates play vital roles in cell metabolic and homeostatic processes. The fourth and final stage of cholesterol synthesis involves the cyclization of the intermediate Squalene into a 4-ringed structure with a steroid nucleus, hydrocarbon side chain, and single alcohol group. This forms the basic structure of cholesterol. 


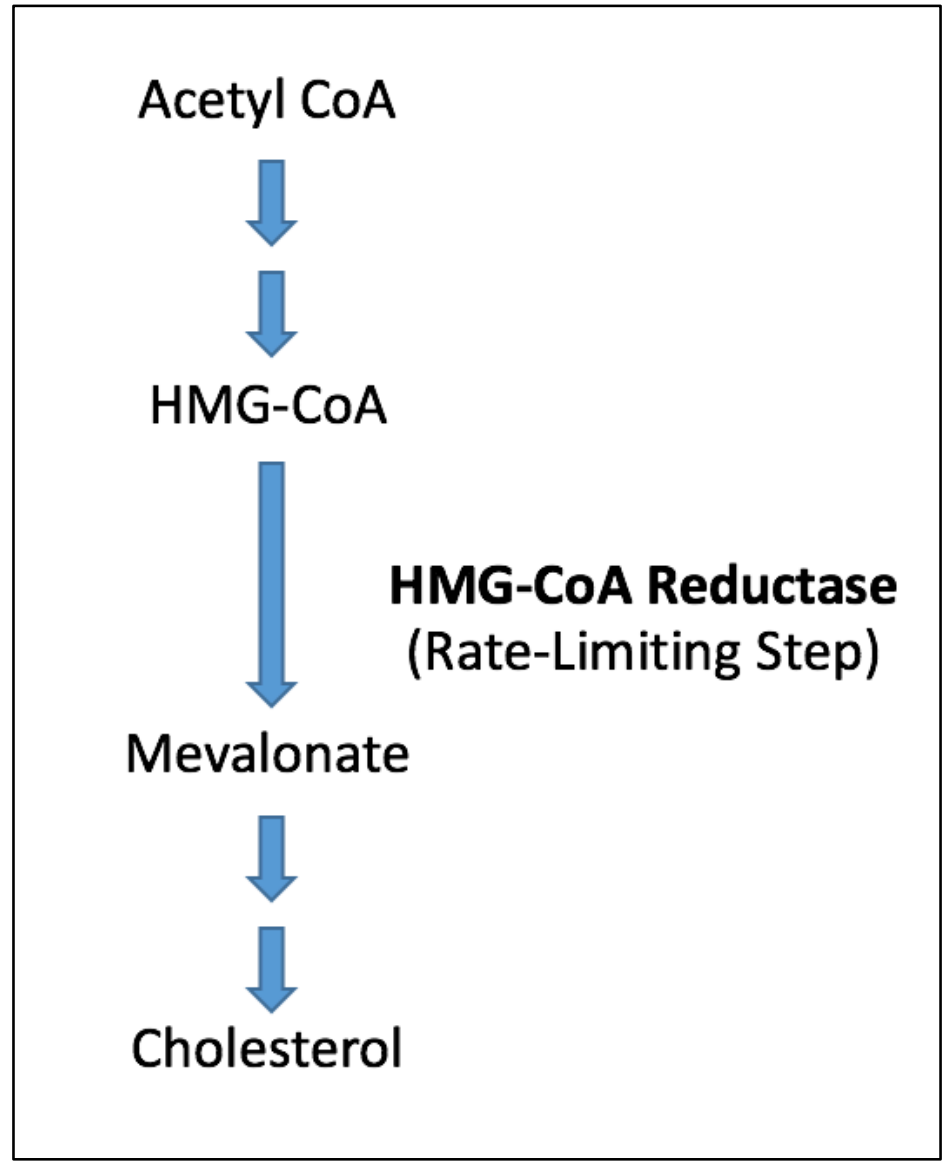

Figure 1. General, conceptualized mechanism of cholesterol synthesis from Acetyl-CoA. 


\section{Cholesterol Transport}

The transport of cholesterol around the body is also another important consideration. For instance, cholesterol is not water-soluble and, therefore, requires a vehicle for transportation $^{12}$. To this end, it is packaged as a cholesterol ester (CE) within one of several lipoproteins, all of which act as cholesterol and triacylglycerol (TAG) transporters from cholesterol-producing cells to peripheral recipients. Enzymes known as acyl CoA-cholesterol acyl transferases (ACAT) or phosphatidylcholine cholesterol acyl transferases (PCAT) take long fatty acid chains and append them to cholesterol molecules, generating CEs. While ACAT acts intracellularly and PCAT acts extracellularly, these are two different enzymes that act at two different sites but ultimately perform the same function - yielding the same CE product. While the bulk of cholesterol within a lipoprotein is stored as $\mathrm{CE}$, the phospholipid monolayer of the lipoprotein itself contains varying proportions of unesterified cholesterol, depending on the identity of the lipoprotein ${ }^{12}$.

There are four main classes of lipoproteins, all of which are complexed with specific carrier proteins known as apolipoproteins ${ }^{12}$. Chylomicrons (typically complexed with APOB48, APOC2, APOC3, and/or APOE) are synthesized in the intestine and are responsible for the transport of dietary cholesterol and triglycerides from the intestines to the liver and other tissues. In this case, since lipids originate from the diet, this pathway is considered to be an exogenous lipid transport pathway. Chylomicrons typically have the highest TAG composition ( $85 \%$ of their weight), but also transport $\mathrm{CE}(3 \% \mathrm{wt})$ and free cholesterol (1\% wt). After the removal of APOC2 and its TAG stores, chylomicrons become significantly smaller and are termed chylomicron remnants, which go back to the 
liver for degradation ${ }^{14}$. Very low-density lipoproteins (VLDL; can be complexed with APO-B100, APOC1, APOC2, APOC3, and APOE) are made in the liver. VLDL serves to transport newly synthesized TAG and cholesterol to the extrahepatic tissues for storage as fat. Since these lipids originate from the liver, the VLDL-mediated pathway is considered to be the endogenous pathway for lipid transport. These lipoproteins hold considerably more CE than chylomicrons (at $12 \% \mathrm{wt}$ ) as well as unmodified cholesterol (7\% wt) but significantly less TAG (50\% wt). Low-density lipoproteins (LDL) are ultimately generated from VLDL by the removal of TAG during VLDL metabolism. LDL contains the highest density of CE (37\% wt) and free cholesterol ( $8 \% \mathrm{wt})$, but the second lowest concentration of TAG $(10 \% \mathrm{wt})$. Lastly, high-density lipoproteins (HDL; complexed with APOC1 and APOC2, but also associated with APOA1, APOA2, APOA4, APOC3, APOD, and APOE) are synthesized in the liver and intestine. In addition to harboring intermediate amounts of CE and free cholesterol (15\% wt and 2\% wt, respectively), HDL actually has a significantly higher density of proteins $(55 \% \mathrm{wt})$ and phospholipids (24\% wt). HDL plays a significant role in a process known as reverse cholesterol transport (RCT), since it primarily collects cholesterol from peripheral locations and transports it back to the liver for processing. It also contains the enzyme LCAT, converting the cholesterol from chylomicrons and VLDL remnants into $\mathrm{CE}^{12}$. All of these lipoproteins play intermediary, interactive roles in a larger cascade.

HDL and LDL are the two most clinically relevant lipoproteins. LDL cholesterol is often termed "bad cholesterol", while HDL cholesterol is often termed "good cholesterol"15. Though this is somewhat simplistic, there is some truth to these viewpoints. First, to physically compare the two molecules, LDL has a diameter of $22 \mathrm{~nm}$ 
and a mass of $3000 \mathrm{kDa}$. It can hold a significant amount of $\mathrm{CE}$, and is associated with a single $500 \mathrm{kDa}$ Apolipoprotein B molecule ${ }^{16}$. HDL, on the other hand, is a much smaller molecule, having a diameter between $5-12 \mathrm{~nm}^{17,18}$ and most commonly associating with a 28kDa Apolipoprotein A molecule ${ }^{19}$. The lipid-protein bonds in LDL are arranged as beta-sheets, making the two components inseparable. These same bonds in HDL form alpha-helical structures, which can freely dissociate and change structure. As cholesterol is one of many energy sources, it requires less energy (i.e., it is easier) for the body to utilize the cholesterol stored in HDL molecules versus LDL molecules. Secondly, higher HDL levels can confer a protective effect on the heart, lowering the risk for heart attack and stroke. This is partly because HDL is thought to have scavenger-like properties that enable it to clear the LDL cholesterol. Liver sinusoidal cells have an abundance of HDL receptors that then facilitate HDL clearance ${ }^{20}$. This said, peripheral tissues largely derive their cholesterol from LDL. Therefore, while excessive LDL can be harmful (discussed below), a moderate amount is beneficial, if not required.

\section{LDL Cholesterol Metabolism and Regulation}

LDL cholesterol and the LDL-R participate in an intricate form of metabolic regulation. In 1977, Brown, Goldstein, and colleagues were the first to describe LDL metabolism as a process involving receptor-mediated endocytosis $(\mathrm{RME})^{21}$. In this process, LDL cholesterol binds to the LDL receptor (LDL-R), a protein that spans the plasma membrane and contains a cysteine-rich Apolipoprotein B binding site. Once endocytosed, LDL cholesterol is contained within an endosome. The endosome merges with a cell lysosome; the CEs within LDL are hydrolyzed, releasing pure cholesterol. The 
cell then utilizes this cholesterol to supplement its plasma membrane or for steroid and hormone production ${ }^{16}$. As for the LDL-R, it can either be recycled back to the cell surface, or broken down and reconstituted by the endoplasmic reticulum (ER). From the ER, the new LDL-R is routed to the cell membrane via the Golgi complex ${ }^{12}$.

While LDL and the LDL-R possess a fairly simple relationship in terms of binding and internalization, the more complicated and fascinating aspect is the precise control that is exerted on LDL-R expression and endogenous cholesterol production. The German scientist Rudolph Schoenheimer and colleagues were the first to describe the existence of an overall cholesterol feedback loop ${ }^{22}$. Schoenheimer placed mice in glass flasks and fed them a sustained cholesterol-infused or control diet over a period of one month. After sacrificing and dissolving the mice in an ether-sodium hydroxide-alcohol steam bath, he measured the total cholesterol content of each flask. Interestingly, mice given a cholesterol-free diet yielded flasks with a higher cholesterol content than those from mice given cholesterol-laden diets. The experiment indicated that animals can generate cholesterol, and stop making cholesterol when it is present in the diet.

In 1975, Brown and Goldstein described LDL-R regulation in human fibroblasts, noting that the receptor was under feedback regulation and influenced by exogenous cholesterol levels in the culture media ${ }^{23}$. In 1986, Brown and Goldstein discovered a feedback suppression mechanism within cells that kept the amount of membrane cholesterol at a constant level. This was, in large part, due to mediation of the cells' endogenous production of cholesterol by $\mathrm{HMGCR}^{24}$, which made enough cholesterol to maintain membrane fluidity. The 1990s and 2000s brought on a greater understanding of the 'cholesterol sensing mechanisms' that drive LDL-R expression and HMGCR activity. 
Yokoyama was the first to describe the existence of the sterol response element binding protein $(\mathrm{SREBP})^{25}$, a transcription factor that resides on the endoplasmic reticulum. Once translated, SREBP binds the protein Scap ${ }^{26-28}$, the protein that detects membrane sterol levels $^{29}$ through direct binding of cholesterol ${ }^{30}$. A deficiency in membrane cholesterol levels induces the SREBP-Scap complex to bind a complex of vesicular proteins known as COPII ${ }^{31}$. COPII facilitates the internalization of SREBP and Scap into the Golgi apparatus, where proteases cleave the SREB proteins into transcriptionally active segments ${ }^{27,28,32}$. These segments then enter the nucleus to initiate LDL-R and $\mathrm{HMGCR}^{33,34}$ gene transcription, ultimately forcing the cell to produce more endogenous cholesterol and scavenge circulating LDL cholesterol with greater efficiency through increased receptor expression. An excess of membrane cholesterol (with cholesterol levels comprising greater than $5 \%$ of total lipids) results in (a) cholesterol binding directly to $\mathrm{Scap}^{30},(\mathrm{~b})$ an alteration in the configurations of $\mathrm{Scap}^{35}$ and $\mathrm{COPII}^{31}$, and (c) the binding of Scap to a COPII binding inhibitor known as Insig ${ }^{36}$.

\section{Implication of Cholesterol in Heart Disease}

The pathophysiology of atherosclerosis has been demonstrated to be intimately dependent on circulating cholesterol levels. While we take it for granted today that higher LDL levels yield atherosclerotic plaques, this direct relationship was unknown to us as recently as a century ago. Here, we examine several key studies that ultimately characterized this interaction.

One of the earliest contributors to our understanding of cholesterol in heart disease was Russian scientist Alexander Ignatowski, who, in 1907, published a study in 
which he fed full-fat milk, eggs, and meat to rabbits in an effort to understand their effects on atherosclerotic development ${ }^{37}$. Five years later, Nicolai Anichkov conducted a similar experiment, substituting the milk, eggs, and meat with boluses of cholesterol ${ }^{38}$. In both cases, Ignatowski and Anichkov observed the development of aortic atherosclerosis and confirmed a relationship between dietary cholesterol and atherogenesis.

In 1953, Ancel Keys, a Mayo Clinic scientist who eventually popularized the relationship between diet and health, began a pioneering study on the epidemiology of myocardial infarction across seven different countries. He demonstrated that those with higher fat intakes exhibited higher levels of serum cholesterol. Because western diets were known to contain more fat than eastern diets, Keys assessed the role of diet in coronary events for Japanese males situated in Japan, Hawaii, and California. Here, he noticed that males in California had a higher fat intake and increased number of coronary events compared to those living in Hawaii and Japan ${ }^{39}$.

In that same decade, John Gofman, a prominent physician and researcher at the University of California, Berkeley, was the first to identify the presence of VLDL, LDL, and HDL in serum by centrifugation ${ }^{40}$. He and E. Virginia Dobbin, a dietician at a local hospital, then demonstrated the link between increased dietary fat intake and the resulting increases in serum LDL cholesterol ${ }^{41}$. Gofman was also the first to study LDL and HDL levels in heart attack patients compared to healthy controls. He demonstrated that heart attack patients had significantly increased LDL and significantly decreased HDL levels compared to their healthy counterparts.

Several other studies have also focused on the role of HDL in cardiovascular dysfunction ${ }^{19}$. Most notably, the Framingham Heart Study (one of the longest, 
continuously running studies) elucidated in 1988 that high levels of HDL cholesterol reduced the risk of death from heart disease ${ }^{42}$. While HDL can potentially mitigate LDL oxidation (discussed below) ${ }^{43-45}$, HDL levels below normal can also independently indicate cardiovascular dysfunction even in the presence of low LDL cholesterol levels ${ }^{46}$. Conversely, a recent study demonstrated that mutations in which HDL was increased did not necessarily confer a cardio-protective effect, suggesting that HDL levels alone may be unable to overcome particular genetically mediated modes of cardiovascular dysfunction ${ }^{47}$. Still, though much of today's therapies focus on therapeutically lowering LDL levels, the use of HDL to target heart disease is under increasing investigation ${ }^{48}$.

In sum, these key studies dictate a critical interplay between HDL and LDL cholesterol levels and their implications in heart disease. Diet plays a critical role in the prevalence of particular cholesterol subtypes. Diets high in fat lead to a greater incidence of LDL-driven coronary events, while HDL plays a more regulatory role and can even have therapeutic benefit in sufficient concentrations. However, since LDL cholesterol is the key driver of atherosclerosis and heart disease, we will next look at the mechanism of its involvement.

\section{Pathophysiology of Atherosclerosis}

Today, we have a direct mechanism for LDL's involvement in forming atherosclerotic plaques. While all blood vessels are lined with endothelial cells, atherosclerosis is a condition that uniquely affects only the arterial endothelium. The development of a fatty streak (the origins of atherosclerotic plaques) can be broken down into roughly seven distinct steps: (1) lipoprotein transport, (2) lipoprotein retention, (3) 
lipoprotein modification, (4) monocyte adherence, (5) monocyte chemotaxis, (6) monocyte differentiation, and (7) the formation of foam cells ${ }^{49}$.

The earliest atherosclerotic lesions, termed 'fatty streaks', are formed at arterial sites that experience significant turbulent blood flow and sustained hemodynamic stress, both of which can damage the lining endothelium. While normal arterial endothelial cells resist the binding of circulating monocytes and other immune cells, a dysfunctional endothelium at these sites serves as a recruiter for such cells by up-regulating the adhesion molecules required for their binding ${ }^{50}$. Furthermore, endothelial dysfunction impairs tight junctions, enhancing permeability and facilitating the entry of LDL into the sub-intimal space ${ }^{51}$. The oxidation of LDL further seeds the lipoprotein beneath the endothelium as well as modify the lysine residues on Apolipoprotein B. Further oxidation leads to an exacerbation of monocyte chemotaxis; more monocyte-macrophages are recruited to the LDL-filled sub-endothelial space and bind the modified Apolipoprotein B using scavenger receptors. Once LDL is internalized, the monocytes take on a foamy appearance and are thusly termed foam cells ${ }^{49}$. Foam cells then secrete a variety of cytokines that promote inflammation, causing resident smooth muscle cells to proliferate and secrete collagen. This results in an enlargement of the fatty streak to a full-fledged plaque, which can severely narrow or completely occlude a vessel, or can rupture and embolize ${ }^{16}$

\section{Familial Hypercholesterolemia and LDL-R Dysfunction}

Though some have elevated LDL cholesterol levels because of poor diets, a significant number have genetic deficiencies that predispose them to high levels of 
circulating LDL and, ultimately, accelerated cardiovascular disease development. One such group of patients is those suffering from Familial Hypercholesterolemia (FH), a disease characterized by pathologically high levels of plasma LDL cholesterol due to dysfunctional LDL-R. Though Brown and Goldstein were the ones who described the existence of the LDL-R in the mid-1970s $\mathrm{s}^{52-54}$ and discovered its regulation in the $1980 \mathrm{~s}^{24}$, Müller was among the first to clinically describe FH in 1938, as an "inborn error of metabolism" that was inherited in an autosomal dominant manner ${ }^{55}$. Further, he demonstrated that the unusually high plasma LDL cholesterol levels in FH resulted in a consequent 20 -fold increased incidence of heart attacks.

It wasn't until many decades later that Khachadurian described the bimodal inheritance of FH in homozygous (have two defective LDL-R alleles, one inherited from each parent) or heterozygous variants (having only one defective LDL-R allele) ${ }^{56}$. Untreated, homozygotes (seen in $\sim 1$ in $10^{6}$ ) exhibit severely elevated cholesterol levels as high as 600-1000 mg/dl, while heterozygotes exhibit more moderate elevations of 300$600 \mathrm{mg} / \mathrm{dl}$. Though the standard assumption is that 1:500 have the heterozygous variant, it is as frequent as 1:200 to $1: 100$ in Scandinavian populations, suggesting that the disease is in fact under-diagnosed ${ }^{57}$. This buildup of cholesterol can have broad systemic effects. In addition to non-lethal symptoms, such as the formation of xanthomas and xanthelasmas (visible collections of cholesterol in the joints and eyes, respectively), patients also exhibit accelerated cardiovascular disease. To this end, untreated homozygotes can die of heart failure as early as their teens, while untreated heterozygotes can die in their $30 \mathrm{~s}$ or $40 \mathrm{~s}^{58,59}$

As stated before, these clinical manifestations are largely due to inherited defects 
in the LDL-R. To date, over 1200 mutations affecting the LDL-R have been identified ${ }^{60}$. These mutations affect LDL-R functionality in at least one of six ways: (1) LDL-R is not made, (2) LDL-R is not transported to the surface, (3) the binding domain of LDL-R is altered, preventing LDL from binding to LDL-R, (4) LDL can bind, but LDL-R cannot be internalized, and (5) LDL-R are not recycled and are rapidly degraded in the lysosome ${ }^{61}$.

However, there are other, much less prevalent, non-LDL-R-mediated modalities that lead to the same clinical presentation as that seen with LDL-R dysfunction-induced FH. For instance, mutations in Apolipoprotein B prevent LDL cholesterol from binding to LDL-R, affecting about $10 \%$ of individuals with $\mathrm{FH}^{62}$. In another example, in 2009 , Horton and colleagues described the role of proprotein convertase subtilisin/kexin type 9 (PCSK9), an enzyme secreted by the liver and other organs ${ }^{63}$. A gain-of-function mutation in PCSK9 allows PCSK9 to bind the LDL-R and prevent receptor cycling after the receptor and ligand have been internalized. In doing so, the number of surface LDL-R decrease and result in an increase in plasma LDL levels similar to those seen with FH. Conversely, a loss-of-function PCSK9 mutation, described by Cohen in 2006, can lead to significant reductions in both LDL levels and heart attacks compared to the general population $^{64}$. To this end, pharmaceutical companies have sought to reproduce this effect with drugs that act as PCSK9 inhibitors.

\section{Current FH Therapies}

There are several pharmacologic agents that are used to treat FH, though none of these therapies offers a definitive cure. The most prominent example are statins, which 
are considered to be a mainstay of $\mathrm{FH}$ therapy and act by blocking the activity of HMGCR and, consequently, cells' endogenous cholesterol production. A better understanding of this comes from reconsideration of the LDL-R and endogenous cholesterol production via a feedback loop (described in the 'LDL Cholesterol Metabolism and Regulation' section above; also see Figure 2). When statins block HMGCR activity, they block endogenous cholesterol production. With the cells unable to produce their own cholesterol, they are forced to scavenge LDL cholesterol to meet their membrane cholesterol requirements. Consequently, their expression of the LDL-R is upregulated, allowing the cells to internalize more LDL through receptor-mediated endocytosis $^{65}$. This, in turn, lowers peripheral, circulating LDL cholesterol levels and imparts a therapeutic effect.

Statins have had a profound impact on cardiovascular therapy and are amongst the most widely prescribed class of drugs on the market today ${ }^{66}$. This, in large part, has to do with the overwhelming evidence showing the dramatic effect of statins on lowering the plasma LDL levels in $\operatorname{dogs}^{67}$, humans ${ }^{68}$, and human FH heterozygote patients ${ }^{69}$ (in whom "a single functional [LDL-R gene] is present and susceptible to activation by cholesterol deprivation" $\left.{ }^{\prime 16}\right)$.

However, statins have some significant drawbacks. Implicit in the description above is the fact that statins require a functional LDL-R in order to elicit any benefit. Thus, homozygous FH patients lack functional LDL-R and, therefore, are minimally benefitted by statins alone ${ }^{70}$ and, at a minimum, require the administration of other supplemental agents. Statins also harbor side effects such as myalgias and can increase the potential for liver damage, digestive problems, and propagation of Type-2 diabetes, 


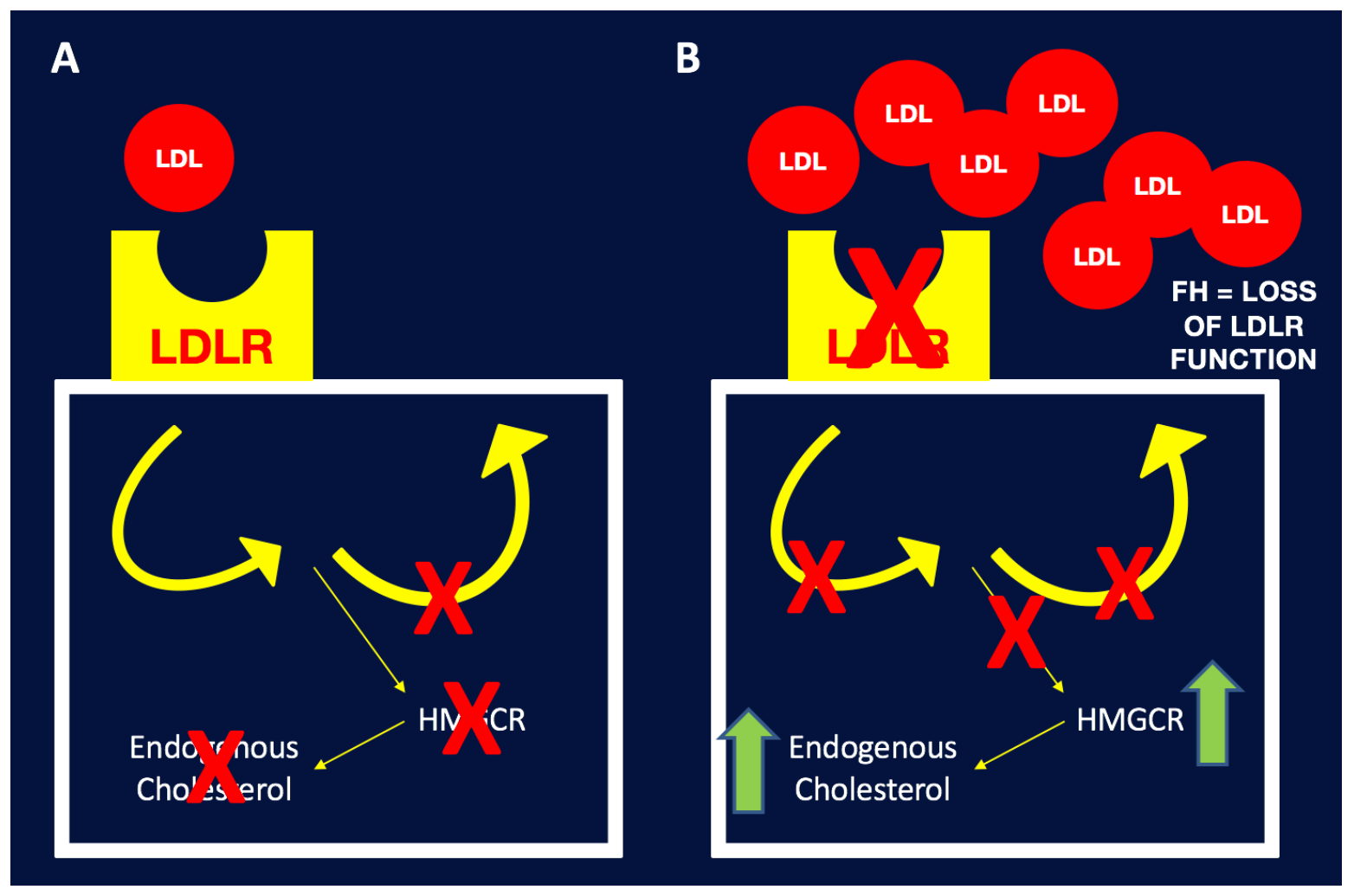

Figure 2. General schematic of the LDL-R feedback loop. (A) In a normal, functional system, the binding of $L D L$ to the $L D L-R$ has an inhibitory effect on the activity of $H M G$ CoA Reductase (HMGCR) within the cell. If the cell obtains enough cholesterol through the $L D L-R$, endogenous cholesterol production ceases and expression of the $L D L-R$ on the cell surface is down-regulated. (B) In FH, the LDL-R is dysfunctional. This dysfunction impairs the negative feedback on HMGCR, leading to increased production of endogenous cholesterol regardless of circulating LDL levels. Statins act to block $H M G C R$ and increase $L D L-R$, but statins only exert a therapeutic effect if $L D L-R$ is functional. Many FH patients have completely dysfunctional LDL-R and are therefore refractory to treatment with statins. 
skin rashes, and neurological side effects.

In many instances, statins are coupled with other drugs to amplify their therapeutic effect ${ }^{2}$. For example, niacin, one of the oldest drugs used for managing circulating lipid levels, has been shown to independently lower LDL cholesterol by up to $20 \%$ while raising HDL levels by as much as $31 \%^{71}$. In another example, PCSK9 inhibitors act to mimic the loss-of-function mutations seen with PCSK9, rendering it unable to prevent LDL-R cycling and consequently making more LDL-R available at the cell surface for binding to LDL cholesterol. In conjunction with statins, PCSK9 inhibitors significantly reduce circulating LDL levels ${ }^{72}$. Third, bile acid sequestrants such as cholestyramine or cholesevelam bind bile in the intestines and prevent their reabsorption via the enterohepatic circulation. Thus, excess bile is removed in the feces. With a shortage of bile in the body, the liver converts excess cholesterol into bile and consequently reduces the amount of cholesterol in the body ${ }^{71}$. As a final example, Ezetimibe is an agent that acts at the intestinal brush border to inhibit cholesterol absorption via lacteals ${ }^{73}$. Though it has significantly reduced LDL cholesterol levels in some trials, other trials have indicated that it can actually increase LDL cholesterol and independently contribute to atherogenesis, negating any potential therapeutic benefit ${ }^{74}$.

Pharmacologic therapies such as these are not completely effective in the face of severe disease. Patients must resort to more invasive options. For instance, homozygous FH patients may require lipoprotein apheresis, a dialysis-like process that physically extricates LDL cholesterol from the blood. However, this approach has several drawbacks. Patients with extremely elevated cholesterol levels may be required to undergo apheresis with increasing frequency. This presents with several challenges, 
namely that (a) the reduction in circulating cholesterol is not permanent (cholesterol rebound), (b) like dialysis, the patient is at risk for infections at the vascular access site, and (c) with the requirement for increased visits, quality of life is reduced while cost of care is increased ${ }^{75}$.

Because liver hepatocytes have the highest density of LDL-R in the body ${ }^{76}$, liver transplant provides the only known cure for FH. In one study, the pediatric recipients of normal LDL-R via liver transplant achieved a clinical resolution of their symptoms and in the case of one patient, even a reversal of their atheromatous lesions ${ }^{77-79}$. However, transplant is not without its own challenges. Hampered by a low availability of quality organs to start with, transplant is also mitigated by non-ideal donor-host compatibility, necessitating lifelong immunosuppression and resulting in an increased susceptibility and adverse response to common infections. Furthermore, transplants are expensive, often costing several hundreds of thousands of dollars. Above all, the procedure itself carries significant risk and can lead to death.

\section{Restoring LDL-R Functionality in FH}

To date, the only definitive cure for FH is liver transplant. However, quality hepatocytes (and liver organs) are in short supply and transplantation has several practical issues. Thus, there are several genetic strategies for mitigating the LDL-R defect present in FH. Broadly put, these efforts rely on either transplanting healthy hepatocytes into patients ${ }^{80-83}$, delivering the corrected LDLR gene directly to hepatocytes (discussion with Wade-Martins as well as ${ }^{84,85}$ ), correcting LDLR in cells ex vivo before their eventual (re)implantation ${ }^{3,86,87}$, or genetically correcting pluripotent cells for eventual implant ${ }^{88}$ or 
differentiation ${ }^{1,89}$.

However, before discussing specific approaches to treating FH, we should first consider the characteristics of these approaches in isolation. Regenerative medicine has significantly advanced due to the advent of gene therapies and transgene delivery ${ }^{90}$. One major driver of gene therapy has been the use of viral vectors, which in general, can infect a wide variety of cell types in an efficient manner via a predictable mechanism. However, viruses have also been associated with significant safety risks ${ }^{91}$. In one case, it has even resulted in death ${ }^{92}$. To understand these dynamics, we will discuss details regarding the four main classes of viral vectors - retroviruses (and lentiviruses), adenoviruses, and adeno-associated viruses.

Retroviruses traditionally replicate their single stranded RNA and convert this into double stranded DNA, which can then integrate into the host genome and utilize host machinery for viral propagation. From this point, viral particles can bud from infected cells and go on to infect other cells. Because retroviruses are naturally highly efficient at performing this process, they are often generated to be replication-deficient for scientific applications to curtail the likelihood of an adverse outcome ${ }^{93}$. Despite this, these viruses still harbor significant limitations. First, they are traditionally not ideal for situations in which transient gene expression is desired, though a more recent effort has highlighted the effectiveness of removing integrase in potentially yielding a transient outcome ${ }^{94}$. In scenarios where a long-term, stable outcome is desired, transgene integration facilitated by viral reverse transcriptase is still required, though adversities have arisen from insertional mutagenesis ${ }^{95}$. Secondly, many retroviruses have difficulty in infecting nondividing cells ${ }^{96-98}$, though lentiviruses (a class of retroviruses) such as HIV-1 can infect 
resting cells ${ }^{99}$ with varying efficiencies ${ }^{100}$. To this end, in treating FH, Grossman and colleagues used retroviral vectors to transduce a functional LDL-R into five FH patients ${ }^{101}$, though only one patient exhibited a significant reduction of LDL levels. This highlights another limitation - efficient retroviral transduction into the liver to sufficiently restore LDL clearance is still challenging ${ }^{102}$. A fourth limitation is that some retroviruses are quite large (upwards of $150 \mathrm{~nm}$ in diameter in some cases) ${ }^{103}$. In the context of the liver, this is important as its vasculature is sinusoidal and fenestrated, and these fenestrations facilitate the transendothelial passage of various biomolecules into parenchymal hepatocytes ${ }^{104,105}$. Additionally, other resident liver cells, such as Kuppfer Cells and the endothelial cells comprising sinusoids (LSEC) can also actively take up various vectors ${ }^{106-108}$. All of these factors serve as barriers that reduce the efficiency of gene therapies to treat genetically dysfunctional hepatocytes.

Adenoviruses have been another option for therapeutic applications. They are desirable as they can carry a relatively large gene payload - for instance, up to $30 \mathrm{~kb}$ of $36 \mathrm{~kb}$ of wild-type adenoviral DNA can be replaced by foreign DNA ${ }^{109,110}$. Though they can be used for transient gene expression, their involvement in inducing host inflammatory responses is not desirable ${ }^{111}$. Though second- and third-generation adenoviruses can offer prolonged transgene expression and reduced immunogenicity ${ }^{112-}$ ${ }^{115}$, more attention has been turned to adeno-associated viral vectors.

Adeno-associated viruses (AAV) are desirable as they are not associated with pathogenicity, exhibited relatively low immunogenicity, and can offer stable, episomal transgene expression ${ }^{116}$. However, their ability to accommodate only small gene packages $(\sim 5 \mathrm{~kb})$ due to their small size $(\sim 22 \mathrm{~nm})^{117}$ means not only are they more ideally suited for 
traversing liver fenestrations, but also that larger genes must delivered in a piecemeal fashion by multiple AAV vectors and re-assembled in vivo by homologous recombination ${ }^{118-120}$. Because all of these pieces have to be delivered to the same cell for the transgene to be functional, this obviously poses an impediment to high efficiency and may require the use of higher viral concentrations. Additionally, several species have preexisting antibodies to AAV, further limiting their efficiency ${ }^{121,122}$. While AAVs recently received European approval for the treatment of familial hyperchylomicronemia (a deficiency of lipoprotein lipase $)^{123}$, AAV delivery to the liver has been shown to elicit liver-tissue damage, likely through a T-cell mediated mechanism ${ }^{124-126}$.

With respect to viral delivery of LDL-R transgenes, though reductions in LDL-C have been reported for relatively short time periods post transfection ${ }^{127,128}$, viral vector LDLR delivery does not yield full amelioration of the FH LDLR deficiency. This is likely because of long-term reductions in transgene expression as well as a diminished degree of vector delivery to target cells $\mathrm{s}^{3,129}$. Additionally, strong promoters that appear to benefit LDL-C regulation in the short term often drive LDLR transgenes. For instance, some have reported no cytotoxicity with constitutive LDLR expression ${ }^{127}$. However, this is controversial since uncontrolled LDLR expression has also been shown to cause lipoprotein cytotoxicity ${ }^{130,131}$.

Hibbitt and colleagues developed a non-viral plasmid ( $p E H Z-L D L R-L D L R)$ containing Epstein-Barr virus OriP and EBNA1 for episomal retention and stable replication with a Hygromycin-B antibiotic selection cassette ${ }^{6}$ (Figure 3). To control the LDLR transgene expression, the plasmid contains $10 \mathrm{~kb}$ of upstream genomic DNA including three sterol response elements for physiological feedback control. In 


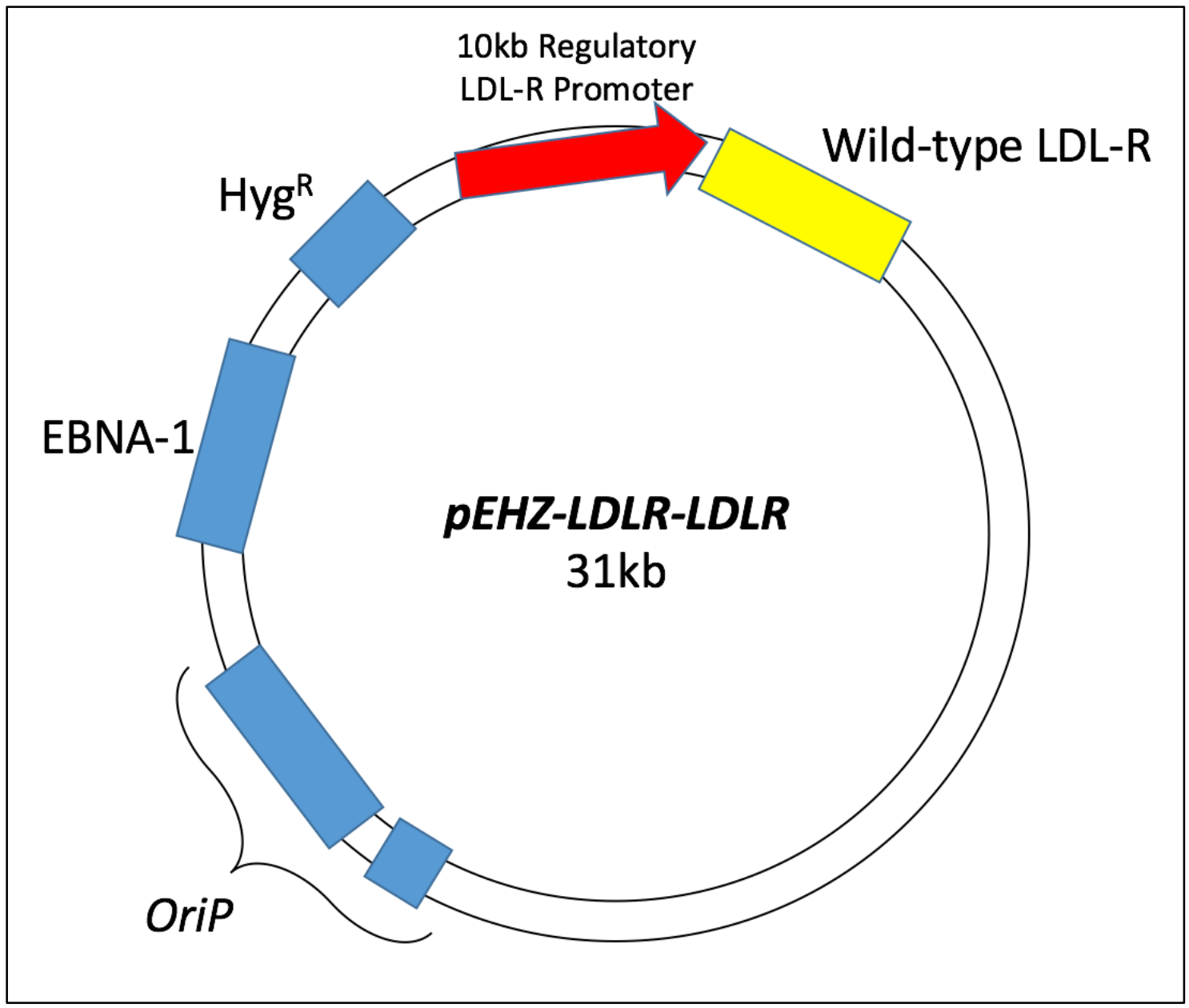

Figure 3. A schematic of the episomal LDL-R-containing plasmid (generated by Hibbitt and colleagues) shows several key features: 1) A wild-type LDL-R gene is preceded by $10 \mathrm{~kb}$ of upstream regulatory control sequences, which house the sterol response elements that confer physiological sensitivity to the plasmid. 2) The plasmid confers Hygromycin-B resistance. 3) Epstein-Barr Viral Nuclear Association factor-1 (EBNA-1) facilitates nuclear localization and maintenance, and is supported by OriP. Note: plasmid is not drawn to scale; locations of various cassettes in this schematic do not reflect their actual position, size, or relation to one another in the plasmid. 
functionality assessments, the plasmid conferred physiologically responsive receptormediated LDL internalization to the $L d l r^{\text {null }}$ CHO-a7 cell line ${ }^{1,6,132}$ and retained an in vivo episomal localization in hepatocytes for 270 days after transfection. These data demonstrate the potential of the $p E H Z-L D L R-L D L R$ system as a gene therapy for treating FH. 


\section{PART II: THE LIVER AND HEPATIC SUBSTITUTES}

As this dissertation project seeks to recapitulate particular aspects of hepatocyte function in the context of a liver-tissue mimic, we should briefly consider the liver anatomy, its cellular organization, major functions, blood supply, and other related functions. However, to best orient ourselves, we will begin with an overview of liver development.

\section{Hepatobiliary Embryological Development}

Our discussion of the liver in the context of cardiovascular dysfunction is particularly fortuitous, as these two systems are intimately related - even in development. There is a considerable degree of interplay between liver and heart growth; in fact, the liver's embryological function is primarily cardiovascular in nature, serving to shunt blood from maternal circulation and umbilical cord directly to the fetal heart and circulation.

To better understand this interplay, consider the interactions between two germ layer derivatives - the primitive ventral endoderm (which gives rise to the liver) and cardiac mesoderm (which gives rise to the heart). The cardiac mesoderm is required for liver formation. In the 1960 s and 70 s, the French scientist Le Douarin studied the interactions between endoderm and mesoderm in developing, chimeric avian embryos. She demonstrated that a piece of primitive gut was unable to develop into a liver by itself, and that hepatogenesis was only achievable in the presence of cardiac mesoderm ${ }^{133}$. 
Furthermore, a juxtacrine interaction between the two developing tissues was required, as the placement of a barrier abrogated the formation of glycogen-storing hepatocytes ${ }^{134}$.

During hepatogenesis, the ventral endoderm tissue expresses a large degree of FGF receptor 4 (FGFR-4) ${ }^{135}$ and FGF receptor 1 (FGFR-1) ${ }^{136}$, while the cardiac mesoderm only expresses FGFR-1 ${ }^{136}$. Of these, FGFR-1 is required for endodermal development ${ }^{137}$. In 1999, Jung demonstrated that FGF-1 and FGF-2 can act as signals that promote liver development ${ }^{138}$. Additionally, FGF-8 promotes outgrowth of the liver bud, though it is not necessary for the induction of hepatogenesis. Interestingly, FGF has a local effect because of its high affinity for the extracellular matrix ${ }^{139}$. This is of particular importance to portions of the ventral endoderm that do not closely oppose the cardiac mesoderm; a lack of local, cardiac mesoderm-derived FGF causes ventral endoderm to develop into pancreatic tissue instead ${ }^{140}$. Therefore, while the ventral endoderm can develop into pancreatic tissue by default, the presence of FGF inhibits pancreatic development and promotes hepatogenesis. Furthermore, this FGF is cardiac mesodermderived $^{141}$. As a second example, the dorsal endoderm can readily differentiate into hepatoblasts in the absence of surrounding tissues. However, in the presence of the dorsal mesoderm, the dorsal endoderm does not undergo hepatogenesis, and instead undergoes gut development. These examples indicate that there are positive and negative differentiation factors that finely modulate hepatic development based on movement and signaling ${ }^{140}$.

Hepatogenesis can be described as a two-step process ${ }^{140}$. We have already discussed the first step, during which cells of the ventral endoderm differentiate down the hepatic lineage. The second step is comprised of the differentiation of hepatoblasts into 
hepatocytes, which depends on additional stimulation by the Septum Transversum (ST) mesenchyme. Mesenchymal interaction may also be necessary for hepatogenesis ${ }^{142}$. The ST is an intermediary cellular layer between the endoderm and cardiac mesoderm that later develops into the epicardium and diaphragm ${ }^{143}$. The ST is also a rich source of BMP4. Multiple studies have demonstrated that the ST, and its abundance of BMP4, are vital to hepatogenesis. Cultures of ventral endoderm sans ST mesenchyme failed to yield a liver. Likewise, $\mathrm{BMP}^{\text {null }}$ mice also failed to develop a liver. Lastly, when both the ventral endoderm, cardiac mesoderm, and ST mesenchyme were all present, the addition of Noggin (a BMP4 antagonist) blocked hepatogenesis and resulted in no albumin expression $^{142}$, even though previous studies demonstrated that FGF alone could drive hepatogenesis ${ }^{138,140}$.

Within this complex milieu of hepatocyte development, hepatic cords begin coalescing around immature endothelial cells known as angioblasts. These cords begin to grow and organize at the expense of the ST, which eventually also forms the liver capsule $^{143}$. The influence of VEGF/Flk1 signaling drives the angioblasts to take on a sinusoidal phenotype; these sinusoids then act as gestation templates for the threedimensional growth of hepatic cords into liver cell plates. At the same time, Notch signaling promotes the differentiation of hepatoblasts into cholangiocytes, the cells of the biliary tree ${ }^{144}$.

In sum, liver development is an incredibly complex process, defining a collaboration between the developing cardiovascular and GI systems. This process is significant to this dissertation project, as the pluripotent stem cells used in our 
experiments undergo similar sequences of differentiation into hepatocytes with several of the same growth factors mentioned above.

\section{Liver: Gross and Microscopic Anatomy}

The human liver is an encapsulated organ that is divided into four key areas, or lobes - the left, right, caudate, and quadrate lobes ${ }^{145}$. These lobes are subdivided into nine segments, all of which are numbered after the nine terminal subdivisions of the portal vein. Approximately $75 \%$ of the liver blood supply is derived from the portal vein (receiving derivatives of the mesenteric veins and arcades), while the hepatic artery (an aortic descendant) provides the remaining $25 \%$. Thus, (1) venous blood from the portal vein is rich in nutrients and (2) blood from the hepatic artery is rich is oxygen. This dual blood supply constitutes the portal circulation ${ }^{145}$.

Microscopically, the liver largely comprised of parenchymal hepatocytes, which are arranged as a series of hexagonal lobules (Figure 4). Each lobule is divided into sixths, with each sixth constituting a liver acinus, the functional unit of the liver (Figure $4)^{145}$. At the vertices of each lobule are trios of vascular and biliary structures collectively referred to as portal triads, each of which contains a hepatic artery, portal vein, and bile duct. A central vein lies at the center of each liver lobule. Given this organization, blood and nutrients travel from the periphery of a hexagonal lobule past all of the hepatocytes within an acinus towards the central vein (Figures 4 and 5). Each lobule is subdivided into three different zones of oxygen and nutrient exposure (Figure 4). Hepatocytes situated within Zone I (periportal zone) are closest to the portal triad and receive the most nutrients, but are also subjected to the highest degree of drug- or toxin-induced damage. 


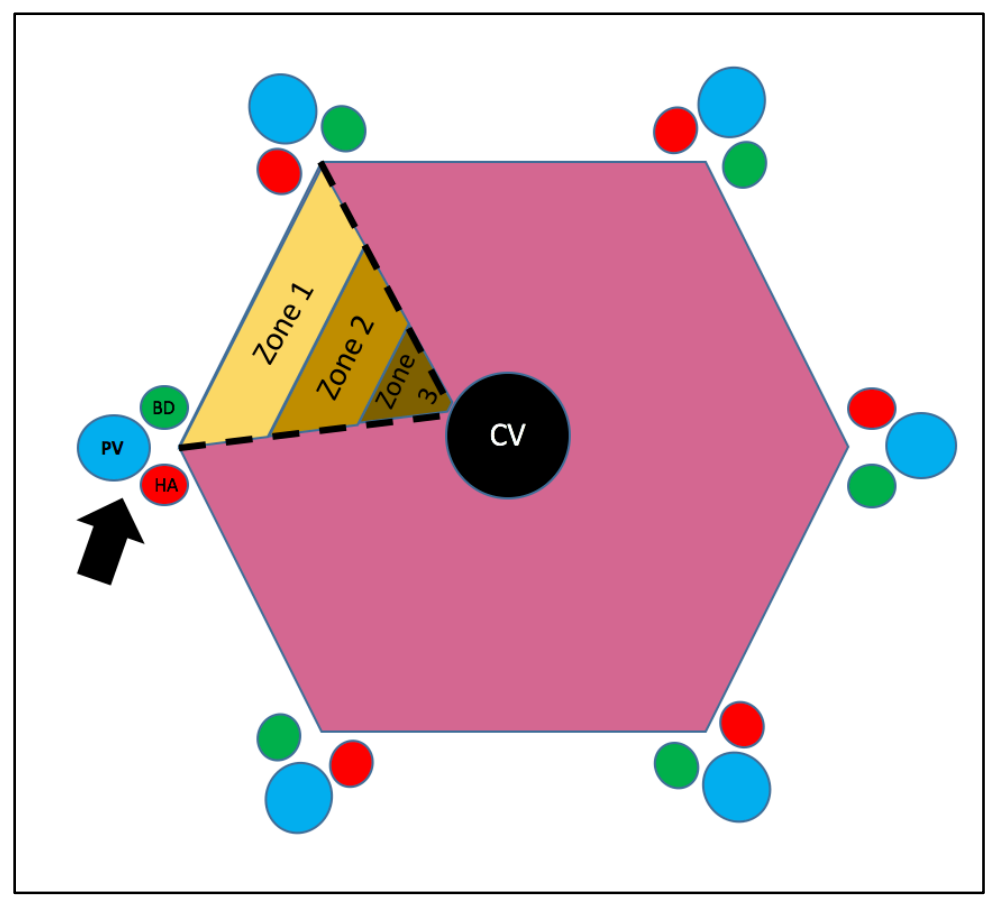

Figure 4. Schematic of a hepatic lobule, with portal triads (or tracts, shown with an arrow), a hepatic acinus (bounded by dashed lines) and zones of oxygenation and nutrient distribution. $P V=$ portal vein, $B D=$ bile duct, $H A=$ hepatic artery, $C V=$ central vein.

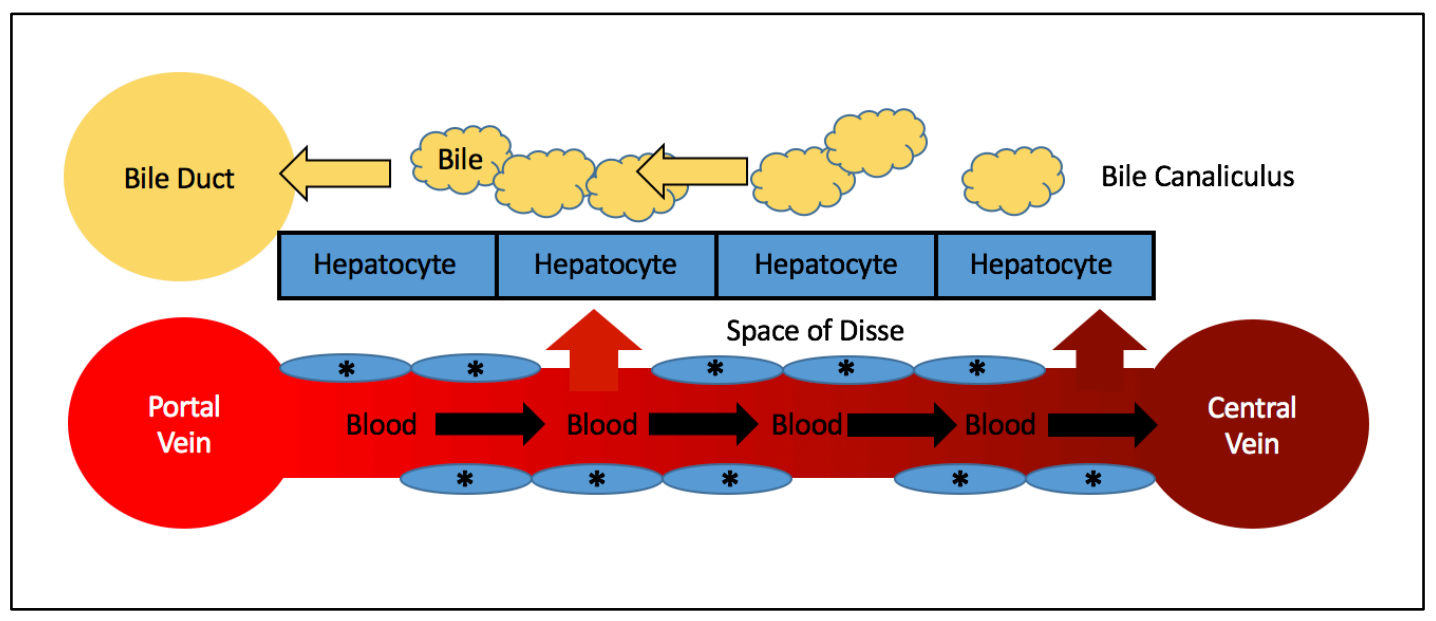

Figure 5. Schematic of fenestrated liver sinusoids lined with endothelial cells (*). Arrows indicate the flow of bile or blood. Also shown is the Space of Disse in-between hepatocytes and the sinusoids. 
Zone II is the area in-between Zones I and III. Zone III (perivenous zone) surrounds the central vein and contains hepatocytes that have minimal oxygen supply but are most resistant to damaging drugs and toxins. From here, the central vein empties into hepatic veins, which drain into the inferior vena cava and returns to the heart.

The liver plays chief roles in cholesterol metabolism (previously discussed), osmotic balance, and the bulk transport of proteins ${ }^{145}$. For all of these processes, the liver requires a unique sinusoidal, fenestrated, discontinuous vasculature (Figure 5). The presence of a discontinuous basement membrane as well as gaps and fenestrations, in the endothelial lining allow large proteins and macromolecules to leave hepatocytes to enter the circulation, and vice versa. To this end, liver sinusoidal endothelial cells (LSEC) are a subset of endothelial cells and are replete with unique identifiers (such as the Mannose receptor, L-SIGN, LYVE-1, VAP-1, and Stabilin-1 and -2$)^{146}$. Sinusoidal vessels link peripheral portal veins with central veins.

Hepatocytes within liver lobules and acini are arranged into sheets of cells that are separated from this sinusoidal vasculature by perisinusoidal voids termed the Spaces of Disse $^{145}$ (Figure 5). Macrophages of the liver, known as Kuppfer cells, have been shown to reside in the Spaces of Disse as well as the sinusoidal lumen ${ }^{147}$. Kuppfer cells target and sequester bloodborne pathogens before sinusoidal blood reaches the surrounding hepatocytes. Hepatocytes, in turn, extend microvilli into this Space to facilitate nutrient absorption. In various pathologies (discussed in detail below), the basement membrane can become continuous and thickened (a process known as capillarization) ${ }^{148,149}$, while the Spaces of Disse are obliterated. Both of these morphological changes detrimentally affect the hepatocytes' ability to obtain nutrients and filter toxins and other metabolites 
from the blood and vice versa.

Hepatic stellate cells (also known as Ito cells) also reside in the Spaces of Disse $^{145}$. The majority of their existence is spent in a quiescent state; their role in this state is still under investigation. Some studies suggest that hepatic stellate cells are liver progenitor cells that mediate liver regeneration and normal cell turnover ${ }^{150-152}$. In instances when the liver is damaged, stellate cells are activated and lay down significant amounts of collagen - similar to the functions of many fibroblasts and perivascular cells - leading to the development of scar tissue and fibrosis.

\section{Liver Dysfunction: Other Examples}

A discussion of the liver would not be complete without briefly acknowledging some other modes of liver dysfunction other than FH. For example, liver failure is a broad classification of organ failure that can lead to systemic dysfunction ${ }^{145}$. As discussed above, because the liver plays a crucial role in cholesterol metabolism, a dysfunctional liver can lead to cardiovascular disease. The liver also acts as a major vascular reservoir and conduit, termed the portal system. In chronic liver failure (taking place over months to years), the microscopic organization of sinusoids and fenestrations undergoes capillarization. Outwardly, portal hypertension ensues, resulting in the development of varices at portacaval anastomoses (connections between liver and systemic circulation) due to the increased pressure. Esophageal varices (swollen, tender blood vessels in the lower esophagus), hemorrhoids (swollen vessels of the rectum), and caput medusae (dilated vessels around the belly button) are consequently exhibited. Additionally, liver hepatocytes are consequently starved of nutrients and oxygen, 
propagating organ failure. However, as the liver filters systemic metabolites and toxins, a failing liver is unable to carry out these functions. Toxins accumulate in the bloodstream and can cause neurological symptoms (such as asterixis (flapping tremors) and mental disorientation and confusion (termed hepatic encephalopathy)) and multi-organ failure ${ }^{145}$.

To contrast, acute liver failure (also known as fulminant liver failure) is sudden, with hyper-acute failure taking place within 1 week, and acute failure occurring within the span of a month. Acute failure accelerates many of the outward symptoms present in chronic liver failure. Cerebral edema and problems with clotting and coagulation are hallmark features of acute disease. Causes of acute failure include drug (e.g., acetaminophen) and alcohol overdose, adverse reactions to particular drugs (such as tetracycline), and viral disease (hepatitis A or B, or Varicella in children leading to Reyes' syndrome) ${ }^{145}$.

In a broader regard, the liver is a major producer of proteins and enzymes. Albumin is a major transport protein produced by the liver that resides in the blood plasma. It also maintains osmotic balance. In liver failure, the production of albumin is significantly reduced. Thus, fluid that would have normally been contained in albuminrich blood is no longer subjected to the same osmotic forces, and consequently collects in the interstitium and perivascular spaces. For this reason, patients with liver failure often present with ascites and edema. In another example, alpha-1 antitrypsin (A1AT) is an enzyme produced by the liver that acts in the lungs. As a protease inhibitor, A1AT protects the lungs from long-term exposure to neutrophil elastase, a local enzyme used for pathogen defense and cell maintenance. A failing liver does not produce sufficient amounts of A1AT, producing symptoms similar to A1AT deficiency, a heritable disorder. 
Here, a lack of A1AT in the lungs results in unmitigated neutrophil elastase. Elastase persists for time periods much longer than normally allowed, leading to destruction of the intricate lung architecture and resulting in lung disease ${ }^{145}$.

In short, these examples are not intended to specifically discuss the full range of complex liver diseases. Rather, they are intended to demonstrate the key functionality of the liver and the systemic consequences of liver failure. Again, this dissertation focuses on the liver from a parenchymal and cardiovascular perspective. However, its key roles with regards to other organ systems are indisputable.

\section{Hepatocyte Cell Sourcing \& Configurations}

The liver is amongst the top organs sought for transplantation. Unfortunately, the demand for high-quality, compatible livers (over 30,000 in the US die annually of liver failure ${ }^{153}$ ) far outstrips its relatively meager supply. Over 16,000 US patients are currently waiting for a liver ${ }^{154}$, and only about $65 \%$ of liver transplant recipients survive after 5 years. Dialysis is an option that is available to kidney failure patients, but because the liver is highly complex and regulates numerous metabolic functions, there are currently few accessible stopgaps to prolong life in the face of liver failure. The major therapeutic option for people with liver failure is transplant. Further complicating care is the fact that the course of liver failure is very hard to predict ${ }^{155}$. These statistics demonstrate the dire need for a personalized, long-lasting, and widely accessible therapeutic system.

Four main biological approaches are being investigated for liver failure; these include hepatocyte transplantation, extracorporeal devices such as the bioartificial liver 
(BAL), xenografts, and implantable constructs ${ }^{155}$. These approaches aim to expedite recovery, promote regeneration, or serve as a bridge-to-transplant. However, there are several considerations. First, hepatocyte transplantation depends on having a sufficient quantity of functional hepatocytes to (a) transplant and (b) elicit a therapeutic outcome. As livers are in short supply, hepatocytes (the liver parenchymal cell) are also in relatively short supply. Though many have attempted to culture and expand primary hepatocytes, these cells are difficult to culture as they depend on the liver microenvironment to survive. As such, in culture, they dedifferentiate and lose their polarity and function over time ${ }^{156,157}$. Others have tried to lengthen in vitro hepatocyte life with enhanced medias ${ }^{158-160}$; however, these specialized formulations are incompatible with patient exposure ${ }^{155}$. Several have developed cell lines (examples include HepG2 ${ }^{161}$ and $\mathrm{C}_{3} \mathrm{~A}^{162}, \mathrm{HepZ}^{163}, \mathrm{HH} 25^{164}$, and $\mathrm{HepaRG}^{165,166}$ ) that closely approximate some primary hepatocyte functions, but these cell lines are either immortalized or tumor-derived. Though several lines have been used in clinical trials, the risk of oncogenic gene transfer is still of concern and hampers widespread clinical application.

Second, non-biological approaches to correct hepatic metabolic deficiencies (hemodialysis, hemoperfusion, and plasmapheresis) are less successful as they only partially and transiently recapitulate the vital functions that whole, healthy livers provide $^{155}$. Extracorporeal devices such as BAL seek to mitigate these shortcomings by adding a hepatocellular element to the device. However, these devices have unique complications such as issues with the transport barrier, scale-up, cell exposure to shear stress, non-uniform perfusion, and cellular stability in suspension. 
Third, xenografts are another source for hepatocytes. Porcine hepatocytes are the most abundant alternative and have been used in pre-clinical and clinical trials ${ }^{167-170}$. Hepatocytes from rabbits, dogs, and rodents have also been used, albeit on a more limited scale. In numerous instances, porcine xenografts incorporated into BAL systems provided measurable therapeutic relief ${ }^{169}$. These xenografts are capable of producing sufficient amounts of albumin, though they express decreased CYP450 activity over time. Further, xenografts are subject to human immunologic reactions and could lead to zoonotic transmission ${ }^{171,172}$. Lastly, hepatocytes are responsive to species-specific signals that are absent in xenograft ${ }^{173}$. All of these factors potentially limit their long-term clinical utility. 


\section{PART III: STEM AND REGENERATIVE CELLS}

As one of the biggest challenges with liver transplant is inadequate supply, stem cells provide a potential avenue for generating the significant cell numbers required for producing enough therapeutic hepatocytes. This section provides a brief overview of stem cells, from embryonic stem cell (ESC) and induced pluripotent stem cell (iPSC) development, to ethical boundaries, to discussing various efforts in producing stem cellderived hepatocyte-like cells.

To begin, we should first acknowledge the differences between totipotent, pluripotent, multipotent, and unipotent cells. Cells that give rise to all embryonic and extraembryonic tissues, they are deemed totipotent ${ }^{174}$. If the cells give rise to only embryonic tissues (these cells are typically sourced from the inner cell mass of a blastocyst (i.e., the second stage of development post-fertilization)), they are termed pluripotent ${ }^{175}$. Adult stem cell populations that give rise to cell lines of related or single lineages are referred to as being unipotent or multipotent, respectively. Lastly, a key consideration with all of these cell designations is that once the cells have fully differentiated down a particular lineage, they lose their ability to differentiate into other tissue types (i.e., their differentiation potential) ${ }^{175}$.

Cloning, another topic common to both ESC and iPSC, was first established by Briggs and King in the $1950 \mathrm{~s}^{176,177}$ and later perfected by John Gurdon in 1960s and $70 \mathrm{~s}^{178}$. In all of these studies, Briggs, King, and Gurdon made the seminal observation that a frog's cells contain the genetic material required to clone entire frogs ${ }^{175}$. Additionally, it demonstrated that differentiated cells are maintained in such a state 
through a series of epigenetic changes, and that these changes could potentially be reversed to force the cells into a de-differentiated state (through a process known as reprogramming). However, simply cloning cells has highlighted problems with genome integrity, ostensibly due to problems with reprogramming ${ }^{178-180}$. For these reasons, cloned cells may be useful for establishing a proof-of-principle but not for more detailed research endeavors.

Experimentation with pluripotent stem cells started in the 1950 s, when cells were taken from mouse germ cell tumors known as teratocarcinomas (or alternatively, embryonic carcinomas ${ }^{181}$ ). These cells could be clonally expanded ${ }^{182}$ and were shown to maintain pluripotence by differentiating into the three embryonic germ layers - the ectoderm, mesoderm, and endoderm ${ }^{183}$. Thus, embryonic carcinoma cells could be used to model aspects of mammalian development.

Embryonic carcinoma cells (ECC) played a pivotal role in ESC and iPSC development in the late 1970s, when Miller and Ruddle demonstrated that the combination of ECC with thymus cells yielded a cell line with ECC properties devoid of all somatic features ${ }^{184,185}$. These studies were critical, as they demonstrated that (a) pluripotent phenotypes exert a dominant influence on differentiated somatic cells, (b) the ECCs contained some transferrable factor that induced pluripotence, and (c) if these factors existed, they should be identifiable ${ }^{175}$.

\section{Embryonic Stem Cells}

The transition from ECC to ESC took place after the implantation of mouse embryos outside the uterus resulted in the development of embryonic carcinomas ${ }^{186}$. This 
finding led to the postulation that embryos themselves might have stem cells. In 1981, Evans and Kaufman ${ }^{187}$ and Martin ${ }^{188}$ successfully isolated mouse ESC (mESC) from blastocysts and developed in vitro culture conditions. These $\mathrm{mESC}$ were able to generate all three germ layers in vivo in spontaneously differentiated, tumor-like structures known as teratomas. A few years later, between 1984 and 1988, the basis for human ESC (hESC) development was formed when Andrews generated pluripotent stem cells (PSC) from a human teratocarcinoma line ${ }^{189}$; later on, clones from this line were exposed to retinoic acid to form neuron-like cells ${ }^{190}$. Pera and colleagues subsequently derived human embryonic carcinoma cells that were able to form all three germ layers, but exhibited chromosomal abnormalities and limitations in their ability to undergo spontaneous differentiation ${ }^{191}$.

The mid 1990s marked key advances in hESC generation. In 1994, human blastocysts were donated by patients for research purposes; the inner cell mass of these blastocysts were able to be cultured for 2 passages, but exhibited normal karyotyping and maintained a stem cell-like morphology (i.e., a bunched appearance with a high nuclearto-cytoplasm ratio) ${ }^{192}$. Isolation of ESC from the blastocysts of rhesus monkeys ${ }^{193}$ and marmosets ${ }^{194}$ provided the first proof-of-concept for what could be expected with human cells. These non-human primate ESC were pluripotent and resembled human embryonic carcinoma cells in vitro. In 1998, Thomson made a significant leap when he and colleagues were able to use donated human blastocysts to derive $\mathrm{ESC}^{195}$. Several nonclonal lines were established, all of which demonstrated pluripotence through teratoma assays. 
In addition to practical issues with $\mathrm{hESC}$ maintenance of a normal karyotype, the long-term maintenance of unique characteristics, and a generalized need for improved differentiation protocols ${ }^{196}$, hESC are also shrouded in ethical controversies that hamper their widespread use in clinical therapies. While hESC are sourced from embryos that develop from eggs that have undergone in vitro fertilization ${ }^{195}$, these eggs are not fertilized in vivo and do not become fully formed fetuses. However, isolation of hESC require the early embryo to be destroyed, leading several to question the existence and degree of a moral status ${ }^{197}$. These challenges prompted researchers to find other avenues of generating pluripotent cells, and in particular, induced pluripotent stem cells.

\section{Induced Pluripotent Stem Cells}

The generation of induced pluripotent stem cells (iPSC) depends on three key principles, namely (a) reprogramming, (b) the derivation and culture of pluripotent stem cell lines, and (c) the observation that transcription factors determine cell fate ${ }^{175}$. With respect to the last principle, several have made the observation that transcription factors associated with a particular lineage could drive pluripotent cells towards that same lineage ${ }^{198-200}$. Furthermore, these studies demonstrated that during lineage-specific differentiation, erroneous factors goading the differentiating cells into inappropriate lineages were automatically stifled. Progressing in a reverse direction, pluripotent stem cells were shown to induce pluripotence in somatic cells after cell fusion, suggesting that there are some transferrable factors that behave in a dominant fashion ${ }^{201,202}$. However, the identity of these factors remained unknown for several years. 
In 2003, Tokuzawa, Yamanaka, and colleagues developed an elegant screening mechanism for determining which transcription factors conferred pluripotence ${ }^{203}$. Using 24 transcription factors associated with pluripotent stem cells, they methodically removed one transcription factor after another, ultimately discovering that a series of four factors (Oct4, Sox2, Klf4, and cMyc) were required to produce colonies that resembled mouse $\mathrm{ESC}^{204}$; these colonies were then termed induced pluripotent stem cells, or iPSC. A year later, the same feat was achieved with human $\operatorname{cells}^{205}$. These discoveries were so monumental that Yamanaka and Gurdon were jointly awarded the Nobel Prize in Medicine in 2012. To date, iPSC have been derived from several species, such as humans, pigs, rodents, and monkeys. In several these species, factors such as OCT4, by itself or in combination with $\mathrm{SOX}^{206} \mathrm{KLF}^{207,208}, \mathrm{cMYC}^{204,209}$, and/or LIN28 and $\mathrm{NANOG}^{210}$, induced pluripotence in a variety of terminally differentiated, somatic tissues, indicating that there are several fundamental features of transcript networks that are evolutionarily conserved.

\section{iPSC and Reprogramming}

As mentioned earlier, the use of factors to convert a terminally differentiated somatic cell into a pluripotent stem cell is termed reprogramming. As the ability to produce iPSC are predicated on the use of the four reprogramming (or "Yamanaka") factors, the modality by which these factors are introduced is equally as important. In this section, we will discuss several methodologies as well as their advantages and any caveats. 
The first efforts in reprogramming utilized constitutively active retroviral vectors. While retroviruses enabled an efficient delivery of the reprogramming transgenes, the transgenes themselves stably integrated into the host genome. Additionally, they could be inactivated by host methyltransferases ${ }^{211,212}$, leading to cell lines that were partially reprogrammed. As the transgenes were integrated, they were also susceptible to reactivation, propagating the formation of tumors in chimeric animal models ${ }^{213}$. To increase the efficiency of transgene delivery, lentiviruses were utilized, though many lentiviruses actually intensified other retroviral shortcomings and led to such robust transgene expression that differentiation was completely impeded ${ }^{214,215}$.

Because inadvertent transgene expression can have undesirable consequences, efforts to mitigate this were subsequently pursued. Inducible lentiviral vectors improved on lentiviral performance by relying on indolent drugs to control transgene expression. For example, when somatic cells were placed in medium containing doxycycline, the reprogramming factors were expressed. Conversely, the removal of doxycycline from the cell medium led to an abrogation of reprogramming transgene expression ${ }^{214,216}$.

A fourth reprogramming modality involves using inducible vectors in a two-step process known as secondary reprogramming ${ }^{217-220}$. In the first step, inducible lentiviral vectors are used on somatic cells as described above. Because the removal of the indolent drug terminates reprogramming, primary iPSC clones can be identified and differentiated into a terminal somatic cell type. As all primary iPSC are exposed to the same differentiation regimen, the resulting somatic cells are, theoretically, genetically identical. Introducing these iPSC-derived somatic cells to the same, indolent drug leads to a reactivation of the reprogramming transgenes, forcing the newly generated somatic cell 
population to uniformly regress into a pluripotent state. This method of reprogramming is advantageous because it facilitates reprogramming on a large scale. Secondly, it enhances efficiency because a homogeneous population is being used (in the second step) to generate an iPSC population (hence the term "secondary reprogramming"). Thirdly, this reprogramming modality permits the comparison of genetically matched iPSC from different somatic cell populations ${ }^{175}$. However, these approaches still result in integration, which can permit transgene activation at any time as well as increase the likelihood for insertional mutagenesis. These pose obstacles for widespread clinical use.

To counter these caveats, several non-integrating reprogramming approaches have recently been developed. Simply put, these approaches fall into one of three classes: (1) a complete obviation of integration, (2) transient integration, or (3) complete avoidance of nucleic acids in the reprogramming vectors. We can similarly assess these three classes as we did with the integrative modalities.

Non-integrating adenoviruses ${ }^{221}$, Sendai viruses ${ }^{222}$, polycistronic mini-circles ${ }^{223}$, and self-replicating episomes ${ }^{224}$ have all been used to reprogram various human fibroblast lines. Because the reprogramming transgenes are not integrated, these modalities provide a unified proof-of-principle that transient transgene expression is enough for producing iPSC. While all of these methods obviate integration, a significant hit is taken with respect to reprogramming efficiency compared to traditional, integrative methods ${ }^{175}$, though reprogramming efficiency is not that significant a concern given iPS cells' propensity to proliferate rapidly once formed.

Another method involves using recombinant vectors that initially integrate into the host genome, but are later removed by the actions of Cre recombinase ${ }^{225}$. This 
approach, known as integration-dependent gene delivery, is particularly advantageous when single mRNAs encoding multiple genes (i.e., polycistronic mRNA) are used ${ }^{226}$. However, the nature of transgene extrication is such that small segments of genetic material (i.e., "footprints") are left behind. The long-term, downstream effects of such footprints are unknown. Similar to integration-dependent gene delivery, Piggybac transposons are mobile genetic elements that can efficiently transpose genetic material between vectors and chromosome in a "cut and paste" fashion ${ }^{227}$. The enzyme Piggybac transposase moves the reprogramming transgene from the donor vector for integration at TTAA sites on the recipient chromosome. After transgene expression occurs for a desired amount of time, an excision Piggybac transposase can be used to fully excise the transgene without leaving behind any foreign genetic material ${ }^{228}$. While this improves on some of the shortcomings associated with integration-dependent gene delivery, sequencing of candidate integration sites is required. Additionally, the effects of Piggybac transposase on the host genome have yet to be investigated.

The next approach is perhaps most analogous to the early experiments in which pluripotent cells were cultured with somatic cells. This led to the hypothesis that there must be some transferable, dominant factor(s) that confers pluripotence. In 2010, Cho and colleagues delivered whole-cell extracts from ESC to somatic cells ${ }^{229}$. While laboratory-directed genetic manipulation was obviated, the methodology was not efficient.

A methodology for delivering synthetic modified RNA (modRNA) was recently, successfully used for iPSC reprogramming ${ }^{230,231}$. The potential advantages of this strategy are that (a) transfection is transient, with a modRNA half-life of 12 hours $^{232}$ and 
(b) being mRNA based, modRNA avoids the possibility of genomic insertion, though it requires repeated transfection with $c-M y c$ which could be potentially problematic ${ }^{233}$. In addition to these facets, modRNA is reported to yield one of the highest reprogramming efficiencies, approaching $2 \%$ in normal fibroblasts ${ }^{234}$.

\section{Challenges with iPSC}

Like ESC, iPSC face several challenges as well, but many of these can be overcome with alternative approaches to their generation and maintenance. However, while ESC and iPSC are similar in their ability to self-renew indefinitely, express genes associated with pluripotency, engage in spontaneous differentiation and teratoma formation, and form live animals ${ }^{235}$, there is some debate as to whether or not iPSC are truly functionally equivalent to $\mathrm{ESC}^{236}$. Several epigenetic control and gene expression patterns are similar between the two cell types ${ }^{213,237-239}$, though iPSC exhibit a propensity for tumor formation (Miura 2009), senesce prematurely ${ }^{240}$, exhibit differences in mRNA $^{241}$ and micro RNA ${ }^{242}$ expression patterns.

Another issue lies with iPSC supposed predilection for differentiating towards particular lineages, namely those of their source cells; in this instance, the cells are described as having epigenetic memory ${ }^{243}$. In 2010, Kim and colleagues sourced iPSC from fibroblast and blood cells, and found that the iPSC exhibited the same DNA methylation patterns as the cells from which they were derived ${ }^{244}$. Ji and colleagues subjected 8 iPSC lines to methylation analysis and determined that the lineage outcome was potentially orchestrated by changes in DNA methylation patterns ${ }^{245}$. To corroborate these findings, that same year, Polo and colleagues looked at a full array of tissues and 
noted that iPSC possessed an altered differentiation capacity compared to $\mathrm{ESC}^{246}$. However, recent findings suggest that these differences may be artifacts of the environments in which the iPSC were generated ${ }^{247}$, and that different labs' emphases may in fact be leaving different signatures on the cells ${ }^{235}$.

When pondering the issue of iPSC-ESC equivalence in light of this information, it is also important to consider that ESC can also be affected in similar ways. ESC have been shown to exhibit lineage propensity ${ }^{248}$ as well as differential gene expression patterns $^{249}$. ESC and iPSC, despite their many similarities, are not absolutely equivalent and are even different from isolation onwards ${ }^{235}$. Therefore, more stringent, standardized methods for generating these cell types need to be developed, or we need to consider other means of overcoming these realities.

Another consideration is that iPSC form teratomas more readily than ESC $^{250}$. While this may not be problematic in fully differentiated cultures, like hESC, the efficiency of iPSC differentiation into terminal cell types has never reached $100 \%$. In fact, efficiencies can vary between $1 \%$ to $80 \%$ depending on the lineage to which the iPSC are being directed ${ }^{251}$, though such variation could be attributed to the particular differentiation protocol being utilized. That said, the presence of even once undifferentiated iPSC in a culture that has $99.9 \%$ differentiation efficiency would, theoretically, be enough to form a teratoma. The US Food and Drug Administration considers iPS cells' propensity for teratoma formation a barrier to clinical adoption. Consequently, this enhances the requirement for highly robust, efficient differentiation protocols as well as measures to remove undifferentiated cells from such cultures. For example, one strategy involves the selective, chemical ablation of only pluripotent stem 
cells in differentiating cultures ${ }^{252,253}$. This approach spares fully differentiated cells and facilitates the elimination of pluripotent cells that can give rise to teratomas, though the long term genetic stability of iPSC-derived cells and tissues remains to be seen. In another example, $\mathrm{Wu}$ and colleagues recently described the development of a caspase-9based safety switch in their mouse iPSC lines ${ }^{254}$. Since their caspase-9 mechanism relies on dimerization to initiate cell suicide, all that is required is that cells be exposed to a chemical dimerizer (in their case, AP1903).

Fifth, cMYC is an oft-present member of traditional reprogramming factor cocktails. Ectopic cMYC is associated with tumorigenicity ${ }^{255}$. While the omission of cMYC from pluripotent transcription factor cocktails do indeed yield $\mathrm{iPSC}^{208,233}$, reprogramming efficiency reduces significantly ${ }^{256}$. Still, if the presence of cMYC is a concern, reprogramming efficiency is less of a concern as iPSC proliferate rapidly once generated; in principle, only one iPS cell is required to then generate larger volumes of cells for downstream applications.

Lastly, another concern for the generation and potential clinical use of iPSC are genomic integration of exogenous reprogramming factors and unwanted spontaneous transcriptional activation post genomic integration, both of which are associated with viral-vector technologies ${ }^{230,231,257}$. To avoid stable integration, DNA-based transgenes have also been delivered by non-integrating plasmids ${ }^{258}$, mini-circles ${ }^{223}$, and Epstein-Barr episomal vectors ${ }^{210}$. However, because the transgene is still DNA-based, spontaneous insertion $^{259,260}$ and oncogenesis ${ }^{232,258,261}$ are still considerations. With DNA-free technologies, the risk of random genomic integration is obviated. This makes approaches such as modRNA particularly attractive. 
In sum, iPSC have a theoretically limitless proliferation and differentiation potential that makes them attractive for cell-based therapies because of difficulties in culturing and correcting cellular dysfunction in primary cells (such as hepatocytes). Current strategies for (a) deriving iPSC from somatic cell sources non-virally, (b) enhancing differentiation efficiency, and (c) preventing tumorigenesis and teratoma formation better position iPSC as a clinically relevant, therapeutic cell source.

\section{$\underline{\text { Stem Cell Differentiation into Hepatocyte-Like Cells }}$}

The use of primary donor hepatocytes in a therapeutic capacity does not assure successful treatment in the face of liver failure ${ }^{262}$. While one obvious challenge in this regard is donor-recipient compatibility, a cirrhotic or diseased liver can provide a suboptimal environment for primary hepatocytes to thrive, let alone function ${ }^{263}$. While several workarounds include optimizing primary hepatocyte placement (for example, in the lymph nodes ${ }^{264}$, pluripotent stem cells offer unique promise in treating liver dysfunction. Despite the aforementioned challenges, pluripotent stem cells can be differentiated to directed cell types. Additionally, since hepatocytes are notoriously difficult to expand in culture, the ability of several types of PSC to rapidly proliferate in culture means that they can be scaled prior to differentiation and use. To this end, several efforts have utilized pluripotent stem cells to generate hepatocyte-like cells (HLC).

In 2009, Song was amongst the first to develop a multi-stage method for differentiating iPSC into HLC. iPSC were first committed to an endodermal lineage before being exposed to a sequence of factors that gradually matured the developing hepatocyte in three subsequent stages ${ }^{265}$. The resulting HLC expressed characteristic 
hepatic markers alpha-fetoprotein (AFP; marker of immature hepatocytes) and albumin (marker of mature hepatocytes) by day 7. By three weeks after the start of differentiation, the HLC were able to participate in glycogen and urea synthesis and exhibited inducible P450 activity. Others have used similar methods to generate HLC from iPSC - for instance, Si-Tayeb and colleagues utilized a four-stage protocol to similar effect ${ }^{266}$, while Ishikawa obviated the use of exogenous factors entirely and relied on iPSC propensity to differentiate down a particular lineage, presumably based on epigenetic memory ${ }^{267}$.

However, Takebe and colleagues were the first to use iPSC-derived HLC to develop a human organoid - more particularly, an iPSC-derived liver-tissue mimic ${ }^{268}$. They first differentiated the iPSC in two stages, first committing the cells to an endoderm lineage before specifying them down a hepatic lineage. The cells were then combined with a stromal support system comprised of mesenchymal stem cells (MSC) and human umbilical vein endothelial cells (HUVEC), as Zaret and colleagues demonstrated that hepatogenesis is facilitated by adequate stromal and vascular support ${ }^{269}$. Upon placing the newly formed iPSC liver buds (iPSC-LB) in vivo atop the mesenteric circulation, the implant anastomosed with and was perfused by the surrounding mesentery. Furthermore, the iPSC-LB were able to rescue drug-induced liver failure. These findings provided us with a broad proof-of-principle that we sought to refine by using corrected, patientspecific iPSC in conjunction with a clinically relevant, adipose-derived vascular support system.

With regards to iPSC-derived cells for the management of FH, work by Cayo and Fattahi were amongst the first to address patient-specific iPSC generation. Cayo and colleagues developed an FH-specific iPSC line using lentiviral-reprogramming vectors. 
Additionally, they were the first to demonstrate that FH-iPSC could be used to form HLC (using the protocol developed by Si-Tayeb ${ }^{266}$ ) and that without correction of the LDLR, these HLC were deficient in their ability to take up LDL cholesterol ${ }^{270}$. Fattahi followed suit by used a retroviral reprogramming vector to generate iPSC from patient-specific fibroblast population ${ }^{89}$ before differentiating the cells using a modified version of a protocol developed by Basma and colleagues ${ }^{271}$. These cells were able to internalize lipids, produce and store glycogen, metabolize Indocyanine green, and uptake circulating LDL after correction via a lentiviral LDLR transgene vector that was constitutively expressed.

Though all of the aforementioned modalities yield HLC, no single assay can determine whether the resultant cells are analogous to primary hepatocytes. Rather, a combination of tests (such as consideration of cellular morphology, gene and protein expression profiles, and functional characterization) can help ${ }^{272}$. Additionally, while HLC are certainly "hepatocyte-like" based on their physical appearance and phenotype, the cells are often immature (as they express significant amounts of AFP) and possess significantly reduced cytochrome P450 activity ${ }^{272}$ compared to primary hepatocytes. Though these differences may be subtle, they pose some challenges for HLCs' clinical applicability and therapeutic value as a standalone system.

Our work in generating FH-iPSC-derived HLC seeks to combine several nonviral, non-integrating innovative technologies to develop a clinically relevant cell therapy. However, the generation of a functional liver tissue mimic does indeed depend on a stromal and vascular supply. Our next section discusses the vasculature and its support system in greater detail. 


\section{PART IV: THE VASCULATURE}

\section{Overview of Vascular Formation, Organization and Function}

A major goal of the studies described in this dissertation is the development of a functional microcirculation in a liver organ mimic using stromal vascular fraction cells derived from adipose tissue. The presence of a vascular supply is critical to organ survival and function. The studies performed utilize in vitro and in vivo experimental models that replicate aspects of blood vessel development observed in embryos as well as adult organisms undergoing physiologic and or pathologic blood vessel development. During embryonic development the blood vessels that invest all organs originate from a primitive tubular structure. These blood islands form through the process of vasculogenesis and are composed of primitive vessel-forming cells known as the hemangioblasts. During subsequent embryogenesis, the blood islands exhibit cellular proliferation, migration and de novo vessel formation from existing vessels, a process known as angiogenesis. Further maturation of vessels through arteriogenesis and venulogenesis results in a hierarchy of structures collectively known as the microcirculation. Strategies to create a new microcirculation from cellular components must incorporate the signals and cues that support embryonic and adult blood vessel formation, as the presence of an operative microvasculature is critical to organ survival and function.

The peripheral vascular system is broadly subdivided into five groups: arteries, arterioles, capillaries, venules, and veins. Arteries and arterioles carry oxygenated blood away from the lungs and heart and deliver it to the organs. Arteries are typically 
comprised of three layers, or "tunics": (1) The tunica intima is the endothelial lining, underneath which there is an internal elastic membrane (IEM). (2) The tunica media comprises the next layer outside the IEM and is composed of smooth muscle, which maintains vascular tonicity. Just around this layer is the external elastic membrane (EEM). (3) The tunica adventitia is the outer layer, comprised of fibroblasts, connective tissue, and perivascular nerves. Arterioles differ from this organization mainly in the extent and orientation of their smooth muscle composition and overall diameter - both are decreased; arterioles serve as bridges between arteries and capillary beds, and arterioles act to regulate the blood flow of the microcirculation. Venules drain blood from capillary beds into larger veins. Veins themselves are similar to arteries in terms of their organization; however, they appear larger and are less rigid in their structure, namely due to their considerably decreased amount of smooth muscle in the medial layers. Additionally, since veins represent the low-pressure side of the circulation, they exhibit valves as part of their intimal layer; these valves rely on surrounding skeletal muscle contractions to propel blood against gravity back to the heart while minimizing backflow.

Capillaries are the intermediate vessels connecting arterioles with venules ${ }^{273}$. They are one cell layer thick and are comprised primarily of endothelial cells. Embryonically formed by vasculogenesis (de novo vascular formation) ${ }^{274-276}$, capillaries have a diameter of approximately 5-10 $\mu \mathrm{m}$ and normally function in conjunction with other capillaries as part of a capillary bed. Depending on an organ's complexity and metabolic requirements, the number and arrangement of beds varies accordingly. This minute organization makes capillaries the ideal sites for gas, nutrient, and waste exchange. Three types of capillaries exist: continuous, fenestrated, and sinusoidal. 
Continuous capillaries are comprised of endothelial cells that maintain tight junctions throughout, as well as a continuous basement membrane. This arrangement provides no room for larger molecules to freely traverse across the endothelial cells, and most transport is restricted to vesicles. The tight endothelial arrangement and continuous basement membrane are integral to the functionality of the blood-brain barrier, for example. To contrast, fenestrated capillaries are different in that they maintain a continuous basement membrane, but feature fenestrations (the Latin fenestra means “window"). These fenestrations are approximately 60-80 nm wide and facilitate small molecule and protein diffusion ${ }^{277}$. Lastly, the sinusoidal capillaries combine a fenestrated endothelial lining with a discontinuous basement membrane. This membrane features large openings $(30-40 \mu \mathrm{m})$ and allow for the passage of red $(\sim 8 \mu \mathrm{m})$ and white $(\sim 25$ $\mu \mathrm{m})$ blood cells as well as large proteins like albumin $(\sim 65 \mathrm{kDa})$. As such, organs such as the liver, spleen, and sites like the bone marrow and lymph nodes are replete with this type of capillary system.

\section{Endothelial Cells}

All blood vessels have an endothelial lining. Thus, vascular endothelial cells (EC) are a critical component of circulatory and systemic health. In addition to serving as a barrier, endothelial cells play active roles in vascular remodeling, the trafficking of hormones, recruitment and binding of neutrophils, fluid filtration (namely in the renal glomerulus), control blood vessel tone (via signaling with surrounding smooth muscle cells), and hemostasis. The cells are also able to adjust their numbers (by proliferating) and orientation (through branching) to better meet tissue metabolic needs ${ }^{273}$. 
EC exert systemic and cellular influence through a variety of factors. For example, vasoactivity is mediated by bradykinin $^{278}$, which can act on EC to balance tissue oxygen levels as well as overall vascular tone and diameter. Using mechanoreceptors to detect changes in cardiac output and, consequently, shear stress, EC can release nitric oxide (NO) via endothelial nitric oxide synthase (eNOS) to modulate the degree to which tissues are perfused ${ }^{279}$. eNOS production is also potentiated by vascular endothelial growth factor (VEGF), serotonin, bradykinin, and adenosine ${ }^{280}$. In addition to promoting vasodilation, $\mathrm{NO}$ also modulates inflammation, oxidative phosphorylation $^{281}$, and thrombosis, all of which serve to maintain relative environmental quiescence ${ }^{282,283}$. EC-produced NO can also affect proliferation efficiency at the transcriptional level, blocking the translocation of NFKB and inhibiting the activity of various cyclins ${ }^{284}$. In addition to NO, EC can also produce endothelin and other prostanoids (potent vasoconstrictors) ${ }^{285}$ and prostacyclins (which also act as vasodilators in a manner independent of NO activity) ${ }^{286}$. Thus, EC are equipped to finely modulate vascular flow in the existing vasculature to affect perfusion and, ultimately, oxygen delivery.

EC can also play a role in vascular remodeling through angiogenesis. Angiogenesis is the process by which new blood vessels are formed from existing ones. A whole host of factors are involved, but VEGF is amongst the most prominent and wellstudied of the angiogenic factors ${ }^{287}$. In vivo, the presence of low oxygen levels triggers the expression of hypoxia inducing factor-1 (HIF-1), which acts to boost local VEGF expression in EC. VEGF that is produced then has a paracrine effect on surrounding endothelium, leading to (1) EC migration towards a VEGF gradient, (2) new EC 
producing proteases to be able to permeate existing extracellular matrices, (3) a proliferation of EC, and (4) the formation of tube-like structures. Once these tubes are perfused, local oxygen levels increase and the driver of HIF-1 signaling is removed in a feedback-looped manner, stopping angiogenesis. In the formation of more complex vessels, such as arterioles and venules, these vessel subtypes primarily depend on the formation of EC tubes and surrounding basement membranes. Depending on environmental requirements, different cell types (such as smooth muscle cells, adventitial cells, and mural cells) are recruited as needed.

The reorganization of cultured endothelium into phenotypic cord-like structures has also been demonstrated in two-dimensional in vitro systems. In 1980, Folkman and Haudenschild reported that EC plated onto a collagen-coated dish formed tubes by 20 days and an extensive network 10 days $\operatorname{later}^{288}$. A few years later, Madri and Williams used endothelial cells from rodent fat pads to demonstrate that connective tissues regulate the endothelial phenotype with respect to micro-anatomical structures, such as tubes ${ }^{289}$. In the presence of interstitial collagen for example, EC predominantly formed a monolayer, whereas with basement membrane collagen, the EC formed tube-like structures. Montesano and colleagues confirmed these findings, and showed that EC cord formation was indeed a collagen matrix-dependent process ${ }^{290}$. While this phenomenon occurs in the absence of other cell types, it yields capillary-like networks that carry no blood. However, it also demonstrates that the absence of blood flow and pressure are not required for basic EC network assembly and architecture ${ }^{9,287}$. The formation of EC cords in vitro could very well provide novel experimental models for understanding analogous processes in vivo. 


\section{The Stromal Vascular Fraction}

The generation of a stable, supportive vascular supply is critical to the development of large volumes of engineered tissue for implant. In normal host tissue, parenchymal cells often require intimate association with circulating factors, proteins, and products via a patent, well-distributed vascular supply. The literature provides evidence of in vivo implanted parenchymal cell death in the absence of a vasculature ${ }^{291-}$ 295. This is largely because oxygen can only diffuse over a very short distance of no more than a few hundred microns. Additionally, depending on the site of implantation, parenchymal cells may not be able to utilize existing host vasculatures ${ }^{296}$. To circumvent these issues, it may be necessary to implant parenchymal cells with vascular support cells.

The adipose-derived stromal vascular fraction (SVF) is a heterogeneous, cell population with tremendous vascularization potential ${ }^{9,297-299}$. SVF has been shown to spontaneously self-assemble into vessel-like networks of cells when placed in culture ${ }^{300}$. In vivo studies have established that adipose SVF, following implantation, forms functional, hierarchical vasculatures that anastomose with host vessels ${ }^{9,299}$ and perfuse implanted parenchyma ${ }^{1,9}$. These findings suggest that SVF may have potential therapeutic vascularization applications toward the accelerated formation of new microvascular structures in ischemic tissues. To this end, SVF has already been shown to

promote revascularization in models of myocardial infarction ${ }^{301,302}$ and peripheral ischemic disease $\mathrm{ig9}^{2903,304}$. 
The first isolations of SVF were conducted by Rodbell and colleagues, who used the enzyme collagenase to digest fat into buoyant adipocytes and a sedimented SVF pellet $^{305-307}$. Though their efforts focused on only the isolated adipocytes, the first studies evaluating the cellular composition of SVF was published in the 1970s, when Wagner and Matthews used Rodbell's fat digestion protocols to isolate and culture endothelial cells from the rodent epididymal fat pads ${ }^{308}$. Additionally, they noted the presence of perivascular cells as a multicellular, heterogeneous population that surrounded the developing vasculature. Later that decade, Van and colleagues isolated and cultured SVF, documenting an overall doubling time of $40-60$ hours $^{309}$. Though they originally thought SVF to be fibroblastic with lipid-storage potential, adipose-derived vasculatures were also initially thought to have mesothelial cells and not endothelial cells ${ }^{310}$, while others have stated that adipose tissue-derived vessels have endothelial cells as their major cell type ${ }^{311}$., though this study utilized omental fat instead of fat acquired from the epididymal fat pads or uterine horn. SVF is now known to have fibroblasts, mesenchymal stem cells, and endothelial cells, as well as smooth muscle cells, mural cells, macrophages, blood cells, and a whole cadre of other stem cell phenotypes ${ }^{9,312,313}$. While this mixed population more closely recapitulates the variety of cells seen in vivo, there is a lack of consensus regarding the specific proportions of these constituents to one another ${ }^{314}$, though Nunes and colleagues demonstrated the relative proportions of endothelial cells $\left(\mathrm{CD} 31^{+}\right)$, monocyte/macrophages $\left(\mathrm{CD} 14^{+}\right)$, multipotent cells $\left(\mathrm{CXCR} 4^{+} /\right.$ $\mathrm{cMet}^{+}$), and perivascular cells (PDGFR-B ${ }^{+}$) in fresh and cultured rat SVF cells ${ }^{9}$.

A fundamental consideration with SVF is that it is heterogeneous and forms vasculatures that closely resemble those seen in vivo. For instance, host vessels in vivo 
are organized such that larger vessels give off smaller branches, which in turn give off smaller branches, so on and so forth. The degree of branching (and consequently, surface area) constitutes a hierarchy that is dictated by local tissue oxygen and nutrient requirements. Hierarchy is a key feature of mature vascular systems ${ }^{315}$. As discussed above, pure EC cultures have a demonstrated ability to form cords and networks in vitro $^{288,290}$. Additionally, Koh demonstrated that the EC in SVF are required for network formation, and that depletion of EC abrogated vascular assembly ${ }^{299}$. However, pure ECderived networks are largely uniform in diameter, lacking a hierarchical organization, and are therefore considered to be immature ${ }^{316,317}$. SVF is unique in that it not only has the EC required for vascular network assembly, but also a full cadre of support cells as well. These support cells are likely required for stabilization and maturation ${ }^{316,318-320}$. Additionally, SVF may be better able to adapt to different therapeutic applications as it is thought to bear a higher degree of plasticity than other vascular cell sources ${ }^{321}$, though cell sourcing from white adipose tissue versus brown adipose tissue yields site-specific differences ${ }^{322}$.

Another point to emphasize is that SVF itself may promote healing. Adipose stem cells (also known as adipose-derived stromal cells; ASC) are considered to be a mesenchymal stem cell (MSC) subpopulation of $\mathrm{SVF}^{323}$. In addition to the fact that ASC appear to be immunoprivileged, these cells have been shown to migrate to sites of injury and release paracrine secretions that reduce inflammation ${ }^{324}$. This trait has been seen with both intraocular injections and tail vein injections, where the former stabilized the progression streptozocin-induced diabetic retinopathy ${ }^{324}$ and the latter mitigated systemic 
inflammation, improved glucose tolerance, preserved pancreatic B-cell mass, and increased $\beta$-cell proliferation in the face of streptozocin treatment ${ }^{325}$.

ASC have also been shown to directly play significant roles with the vasculature. For instance, Cai and colleagues were amongst the first to demonstrate that ASC impact capillary density and perfusion, and that these actions are mediated by hepatocyte growth factor $(\mathrm{HGF})^{326}$. When placed with human microvessel endothelial cells, ASC improved endothelial network assembly and stability ${ }^{298}$. In trying to determine a mechanism for these outcomes, initial analyses revealed that these cells were positive for a full cadre of markers associated with stromal cells such as mesenchymal cells (CD10, CD13, CD90), pericytes (CD140a/b), and smooth muscle cells (alpha actin, calponin, and caldesmon). Immunohistochemical analysis of ASC-microvessel endothelial cell co-cultures showed that after extensive culturing, the cells encase the vascular lumen in much the same way as pericytes would. Again, determining the density of ASC per SVF isolation is difficult since specific markers have not been identified, though others such as PDGF-B and 3G5 $5^{298,313,327-329}$ and Pref1 ${ }^{330}$ have been implicated. Additionally, since ASC exhibit variable abundance - white adipose tissue is enriched with ASC, for example $e^{322,323}-$ the sourcing of SVF and its subpopulations need to be taken into consideration.

To determine a mechanism of interaction between ASC and endothelial cells, Merfeld-Clauss and colleagues performed a series of co-culture experiments, noting increases in overall extracellular matrix production, ASC expression of alpha smooth muscle actin, and endothelial expression of $\mathrm{CD} 31^{331}$. Co-cultures yielded the highest degree of endothelial network formation, followed by ASC-conditioned media with the next highest density and ASC-fibroblast co-cultures with the lowest density. Not only do 
these findings suggest that ASC-endothelial cell interactions are contact dependent, but also that these interactions progress in both directions (as ASC increased aSMA expression as well). This is evidenced in later studies, implicating Activin A as an ASCendothelial cell contact-initiated means of (a) inducing ASC smooth muscle cell differentiation $^{300}$ and (b) impairing VEGF expression, reducing endothelial network formation $^{332}$. Thus, there is a complex interplay between ASC and endothelial cells. A key takeaway is that ASC are a subpopulation of SVF, and SVF is also replete with endothelial cells ${ }^{9}$. Thus, there is reason to suspect that these mechanisms and others are already at play within this heterogeneous population.

A third, clinical and translational, consideration with SVF is that it is easily isolatable. Adipose tissue can be easily acquired through minimally invasive means and subjected to enzymatic digestion, usually with Collagenase. The resulting digest can then be centrifuged and separated into three distinct layers: (1) adipocytes, which are buoyant, (2) collagenase solution, tumescent fluid, washing buffers, and cell debris, and (3) the SVF cell pellet at the bottom of the centrifuge tube. Though these steps can be performed manually, there is a growing interest in automating them ${ }^{333,334}$ for rapid application at the bedside or during an operation ${ }^{335}$. Further, such automation enhances the likelihood of a desirable, repeatable outcome and may mitigate the effects of lab-to-lab variations.

In short, SVF contains a multitude of cell types that not only promote vascular networking, but vascular support as well. Additionally, SVF's relative ease of isolation makes it a clinically relevant therapeutic cell source for vascularization applications. 
CHAPTER III

\section{GENERATION OF A FUNCTIONAL LIVER-TISSUE MIMIC USING ADIPOSE STROMAL VASCULAR FRACTION CELL-DERIVED VASCULATURES}

\section{OVERVIEW}

A major challenge with therapeutic cell implantation is achieving microcirculatory integration between the implanted cells and host vasculature. Adiposederived stromal vascular fraction (SVF) cells were used vascularize an implant comprised of HepG2 human hepatocyte analogue cells. We hypothesized that the SVF cells would form a functional microcirculation via vascular assembly and inosculation with the host vasculature. Initially, we assessed the extent and character of neovasculatures formed by freshly isolated and cultured SVF cells and found that freshly isolated cells have a higher vascularization potential. Generation of a 3D implant containing fresh SVF and HepG2 cells formed a tissue in which HepG2 cells were entwined with a network of microvessels. Implanted HepG2 cells sequestered labeled LDL delivered by systemic intravascular injection only in SVF-vascularized implants demonstrating that SVF cellderived vasculatures can effectively integrate with host vessels and interface with parenchymal cells to form a functional tissue mimic. 


\section{INTRODUCTION}

Tissue replacement is a strategy that can be used for the regeneration of different organs in scenarios such as organ failure. Cell transplantation offers an alternative to treat patients with organ failure, such as in liver diseases ${ }^{336,337}$. However, minimal engraftment is achieved with this approach ${ }^{336,338,339}$. One of the major caveats in tissue replacement therapies is to promote effective vascularization of the transplanted tissue in order to prevent death and promote engraftment of transplanted cells. Several approaches have been utilized in an attempt to promote vascularization of implanted tissues such as the delivery of angiogenic growth factors to recruit host vessels or co-implantation of endothelial and angiogenic signaling cells with target tissue cells (reviewed in ${ }^{340,341}$ ). Although considerable progress has been achieved, significant obstacles still exist, such as the short half-life of growth factors in the tissues resulting in regression of newly formed vasculatures ${ }^{342,343}$ and potential sources of endothelial and angiogenic signaling cells for human transplants. Adipose-derived stromal vascular fraction (SVF) cells are an attractive cell population for transplantation studies since human adipose tissue is an easily accessible and dispensable tissue source that can provide large numbers of cells suitable for implantation with little donor morbidity and patient discomfort. In addition, SVF cell preparations have been shown to be safely and effectively transplanted to either an autologous or allogeneic host and can be manufactured in accordance with Good Manufacturing/Tissue Practice guidelines ${ }^{314}$. SVF cells are obtained from the enzymatic digestion of adipose tissue to single cells followed by discarding adipocytes. They are a mix of heterogeneous cell populations composed of endothelial cells, fibroblasts, perivascular cells, immune cells and undefined stem cell sub-populations ${ }^{314,319,344}$. The 
potential of SVF cells to promote vascularization and improve organ function when delivered to sites of ischemia has been demonstrated in animal models of peripheral ischemic disease $\mathrm{e}^{299,303,304}$ and myocardial infarction ${ }^{301,302}$. Here, our goal was to harness the vascularization potential of SVF cells in vivo to generate an effective vascular interface between host and transplanted liver cells that results in a functional tissue mimic. We show that (1) adipose-derived SVF cells have a potent intrinsic vascularizing potential, (2) culturing freshly isolated SVF cells retains this vascularization potential despite possible changes in cell populations, and (3) SVF cell-derived vasculatures form a functional interface between host and implanted parenchymal cells.

\section{MATERIALS \& METHODS}

Ethics statement. All animal experiments were performed in compliance with institutional guidelines, as per US National Institutes of Health Guide for the Care and Use of Laboratory Animals and were approved by University of Louisville Institutional Animal Care and Use Committee procedures and policies (IACUC \#12059, 12060).

Rodent and human SVF isolation. Adipose-derived SVF cells were isolated from the epididymal fat pads of male, retired breeder Sprague-Dawley rats (Charles River) under anesthesia [ketamine $(40-80 \mathrm{mg} / \mathrm{kg})$ and xylazine $(5-10 \mathrm{mg} / \mathrm{kg})]$. Green fluorescent protein (GFP)-tagged SVF were obtained from Sprague-Dawley rats that ubiquitously express GFP (Rat Research and Resource Center, University of Missouri, Columbia, MO). Human SVF were isolated from adipose tissue obtained from abdominoplasty (IRB Exempt, \#09.0037). Harvested fat was washed in 0.1\% BSA-PBS, finely minced, and digested in $2 \mathrm{mg} / \mathrm{ml}$ type I collagenase solution (Worthington 
Biochemical Company, Freehold, NJ, USA) for $40 \mathrm{~min}$ at $37^{\circ} \mathrm{C}$ with vigorous shaking. Adipocytes were removed by centrifugation, and the entire cell pellet was washed with 0.1\% BSA-PBS. Cells were either immediately used (Fresh SVF, fSVF) or plated into gelatin-coated plates (Cultured SVF, cSVF $5 \times 10^{4}$ cells $/ \mathrm{cm}^{2}$ ) in fresh media (DMEM supplemented with $2 \mathrm{mM} \mathrm{L-glutamine,} 50 \mu \mathrm{g} / \mathrm{ml}$ ECGS and 10\% FBS). Cultured SVF were used at P0 after 5-7 days when cells reached confluence.

Flow cytometry analysis. cSVF were dissociated with non-enzymatic Cell Dissociation Buffer (Sigma) after reaching confluence (P0) and fixed with 4\% paraformaldehyde for $10 \mathrm{~min}$ at room temperature. Cells were blocked with PBS containing 5\% fetal bovine serum (FBS) for 30 minutes on ice and incubated with the following antibodies in blocking buffer on ice for 1 hour: anti-CD14 (1:100), anti-CD31APC (1:500)(BD Biosciences); anti-cKit (1:100, Abcam); anti-CXCR4 (1:100, Ebiosciences); anti-c-Met (1:100), anti-PDGFRb (1:100, Santa Cruz Biotechnology) overnight at $4^{\circ} \mathrm{C}$. Secondary antibodies used were anti-mouse-Alexa Fluor 488 (1:400) (Jackson ImmunoResearch) and anti-rabbit-Cy6 (1:500) (Jackson ImmunoResearch) for $30 \min$ at $4^{\circ} \mathrm{C}$.

Microvessel isolation. Fat-derived microvessels (FMF) were isolated from rat epididymal fat by limited collagenase digestion and selective screening as previously described $^{345,346}$. The collagenase used (type I; Worthington Biochemical Company, Freehold, NJ, USA) was lot tested to yield high numbers of fragments with intact morphologies. These vessel fragments have the potential to form a microcirculation composed of different vessel types 4 weeks post implantation in vivo in 3-dimensional collagen gels ${ }^{347}$. 
HepG2 cell culture. HepG2 cells were cultured in T-75 tissue culture flasks in HepG2 growth media consisting of Dulbecco's Modified Eagle's Media high glucose, $10 \%$ fetal bovine serum, $1 \mathrm{X}$ penicillin/streptomycin, and $1 \mathrm{X}$ L-glutamine (Invitrogen Camarillo, CA, USA). Media was changed every other day and cells were grown to confluence at which time they were prepared for Cytodex-3 culture as described below. Plasmids and Cell Transduction HepG2 were transduced with retrovirus to constitutively express GFP (pBMN-I-GFP) or Ds-Red as previously described ${ }^{348}$.

Cytodex-3 cell culture. $50 \mathrm{mg}$ of Cytodex-3 microcarrier beads (Sigma, St. Louis, MO, USA) were hydrated with $5 \mathrm{~mL}$ phosphate buffered saline (PBS) $-\mathrm{Ca}^{2+} / \mathrm{Mg}^{2+}$ (Hyclone) for four hours with occasional mixing to avoid aggregation ${ }^{349,350}$. PBS solution was removed and washed out with freshly prepared $70 \%$ ethanol for a total of four washes. The last $70 \%$ ethanol wash was carried overnight. The following day, ethanol was removed and $10 \mathrm{~mL}$ of $\mathrm{HepG} 2$ growth media was added for a total of four washes. The last wash was removed and HepG2 cells were passaged into a resuspension of $1 \mathrm{x}$ $10^{6}$ cells $/ \mathrm{mL} . \quad 6 \times 10^{6}$ cells were added to $4 \mathrm{~mL}$ of HepG2 media containing Cytodex-3 beads and were gently mixed. The bead-cell mixture was added to a $100 \mathrm{~mm}$ petri dish (BD Falcon) and incubated for three days at $37^{\circ} \mathrm{C}$ and $5 \% \mathrm{CO}_{2}$ for optimal microcarrier coverage.

Preparation of 3-dimensional constructs and in vivo implantation. To form the $3 \mathrm{D}$ constructs, fresh or cultured SVF $\left(10^{6}\right.$ cells $\left./ \mathrm{mL}\right)$ were suspended into $3 \mathrm{mg} / \mathrm{mL}$ of collagen type I (BD Biosciences, San Jose, CA, USA) and $0.2 \mathrm{~mL}$ of the suspension was seeded into wells of 48-well culture plates. Constructs were implanted subcutaneously on the flanks of Rag1 mice as previously described ${ }^{351}$. To assess the potential of fresh and 
cultured SVF to participate in the neovascularization process, fresh or cultured SVF from GFP rats $\left(10^{6}\right.$ cells $\left./ \mathrm{mL}\right)$ were seeded into collagen gels concomitantly with isolated FMFs $(20,000 \mathrm{~mL}) . \mathrm{FMF} / \mathrm{SVF} /$ collagen suspensions were pipetted into wells of a 48 -well culture plate $(0.2 \mathrm{~mL} /$ well $)$ to form a $3 \mathrm{D}$ construct that were either cultured in DMEM + $10 \%$ FBS or implanted subcutaneously on the flanks of Rag1 mice as previously described $^{352}$. Alternatively, SVF were seeded in the presence of HepG2 cells before implantation.

Construct analysis. Microvascular constructs were harvested at either 4 or 6 weeks after implantation and fixed in 4\% paraformaldehyde for 20 minutes. Samples were permeabilized with $0.5 \%$ Triton X-100 and rinsed with PBS. After blocking for two hours with $10 \%$ goat serum (Sigma), samples were incubated overnight with fluorescent or biotin conjugated lectins. Following three 15-minute washes in divalent cation-free (DCF)-PBS, samples were imaged en bloc with an Olympus MPE FV1000 Confocal Microscope and analyzed with Amira 5.2 (Visage Imaging, Inc., San Diego, CA, USA). SVF cells were identified by either constitutive expression of GFP (when obtained from animals that ubiquitously and constitutively express GFP) or labeling with TRITC/Fluorescein conjugated or Cy5-streptavidin GSI (rodent SVF) or UEAI (human SVF) lectin (Vector labs, Burlingame, CA, USA). To evaluate vessel perfusion in the implanted constructs, host mice were perfused intravenously with Dextran-TRITC 2,000,000 MW for 15 minutes before the constructs were harvested. Confocal microscopy images with HepG2-GFP+ clusters were identified and examined for presence of GS1-Cy5+ vasculature, DiI-LDL, or both, and recorded in Microsoft Excel. Those images without HepG2-GFP+ clusters were not included. In addition, clusters void 
of both GS1-Cy5+ vasculature and DiI-LDL were recorded. The number of HepG2 clusters associated with each variable were counted and graphed using SigmaPlot. To determine if DiI-LDL uptake is correlated with the presence of GS1-Cy5+ vasculature, HepG2-GFP+ clusters positive for DiI-LDL were plotted against those clusters positive for GS1-Cy5 vasculature. The Pearson correlation coefficient was then calculated for statistical correlation between the two variables.

\section{RESULTS}

Cultured and fresh rodent SVF cells form vessels that are perfused. The development of a functional organ-tissue mimic relies not only on parenchymal cells, but also a vasculature that links this implant with the surrounding vasculature. The stromal vascular fraction (SVF) is an adipose-derived, heterogeneous single cell suspension that has been shown to inosculate with the host vasculature after implantation. SVF cells have also demonstrated vessel-like properties in culture as well. Therefore, fresh and cultured SVF (fSVF and cSVF, respectively) have therapeutic promise in vascularization applications. In this aim, we hypothesized that we could use SVF to functionally vascularize implanted hepatocytes, thereby forming a liver-tissue mimic. To start, using SVF derived from rats constitutively expressing GFP, we were able to assess the nature and degree of interaction between SVF-derived vessels, implanted parenchymal cells, and the native host vasculature (Figure 6). In 3D collagen gel matrices, both fSVF and cSVF spontaneously assembled into perfused, vascular networks. In both populations, GFP ${ }^{+}$ cells were seen contributing to hierarchical, organized vasculatures reminiscent of mature vascular structures. Despite these similarities, there were also many differences. For 


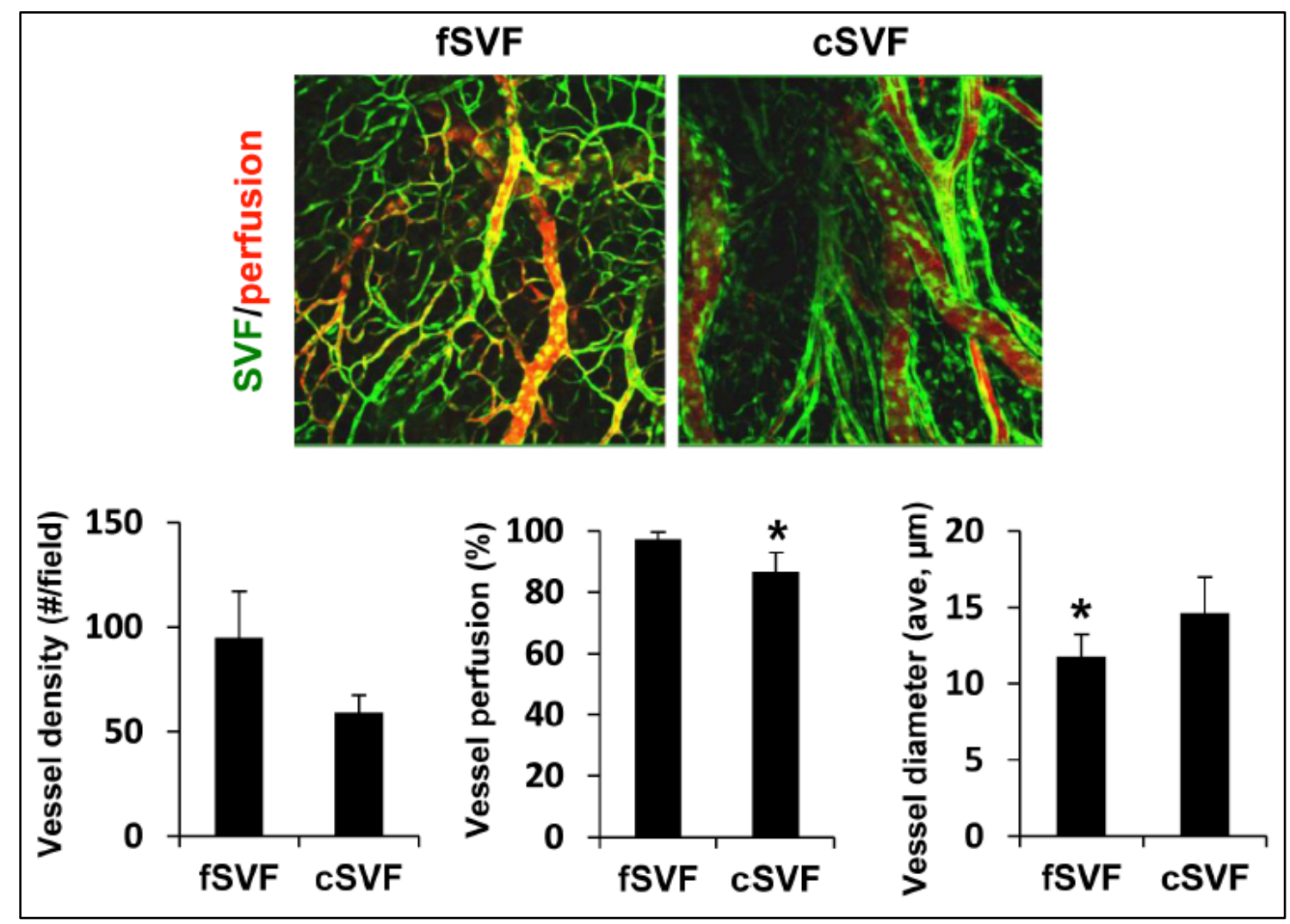

Figure 6. Fresh and cultured SVF cells from GFP-transgenic rats were seeded in 3D collagen matrices and implanted for 4 weeks. Prior to explantation, host animals were perfused with dextran-TRITC. Here, we see that cultured cells are significantly impaired in their perfusion $\left({ }^{*} p=0.001\right)$ and have significantly increased diameters $\left({ }^{*} p=0.02\right)$ compared to fresh SVF isolates. Results shown as mean \pm S.E.M. 
instance, cSVF appeared to form a lower density of vessels when compared to fSVF. In a particular field of view, cSVF generated $59.2 \pm 8$ vessels, while fSVF generated considerably more $-94.2 \pm 22$. Additionally, there was significantly lower perfusion in cSVF-derived vessels as opposed to fSVF-derived vessels. To this end, $97.4 \pm 0.8 \%$ of fSVF-derived vessels were perfused, versus the $86.7 \pm 1.9 \%$ seen with cSVF. Furthermore, differences could be ascertained with the average vessel diameters of the two populations, with cSVF yielding a larger diameter $(14.6 \pm 2.3 \mu \mathrm{m})$ vs. fSVF $(11.7 \pm$ $1.5 \mu \mathrm{m})$. In sum, these results broadly indicate that both cSVF and fSVF possess a proclivity to produce vessels with a similar organization. However, the two populations differ with respect to the magnitudes of various, quantifiable vascular parameters.

\section{Cultured and fresh rodent SVF cells participate in angiogenesis in different}

ways. We have shown that cSVF and fSVF can form perfused, hierarchical vessels in vivo. However, the next question that we asked was whether or not SVF-derived vessels could incorporate into an angiogenic bed. To test this, we relied on an angiogenic microvessel implant model, again comparing fSVF and cSVF cells.

Both cell types took active roles in forming new vessels through angiogenesis (Figure 7). To provide some context, early on, angiogenesis is defined by the formation of immature vascular networks. Here, we observed SVF intimately associating with the endothelial cells of this developing neovascular system. In implants assessed at later time points, we observed vascular systems that were a hybrid of $\mathrm{GFP}^{+}$and $\mathrm{GFP}^{-}$cells, indicating that these vascular systems were chimeric in nature. 


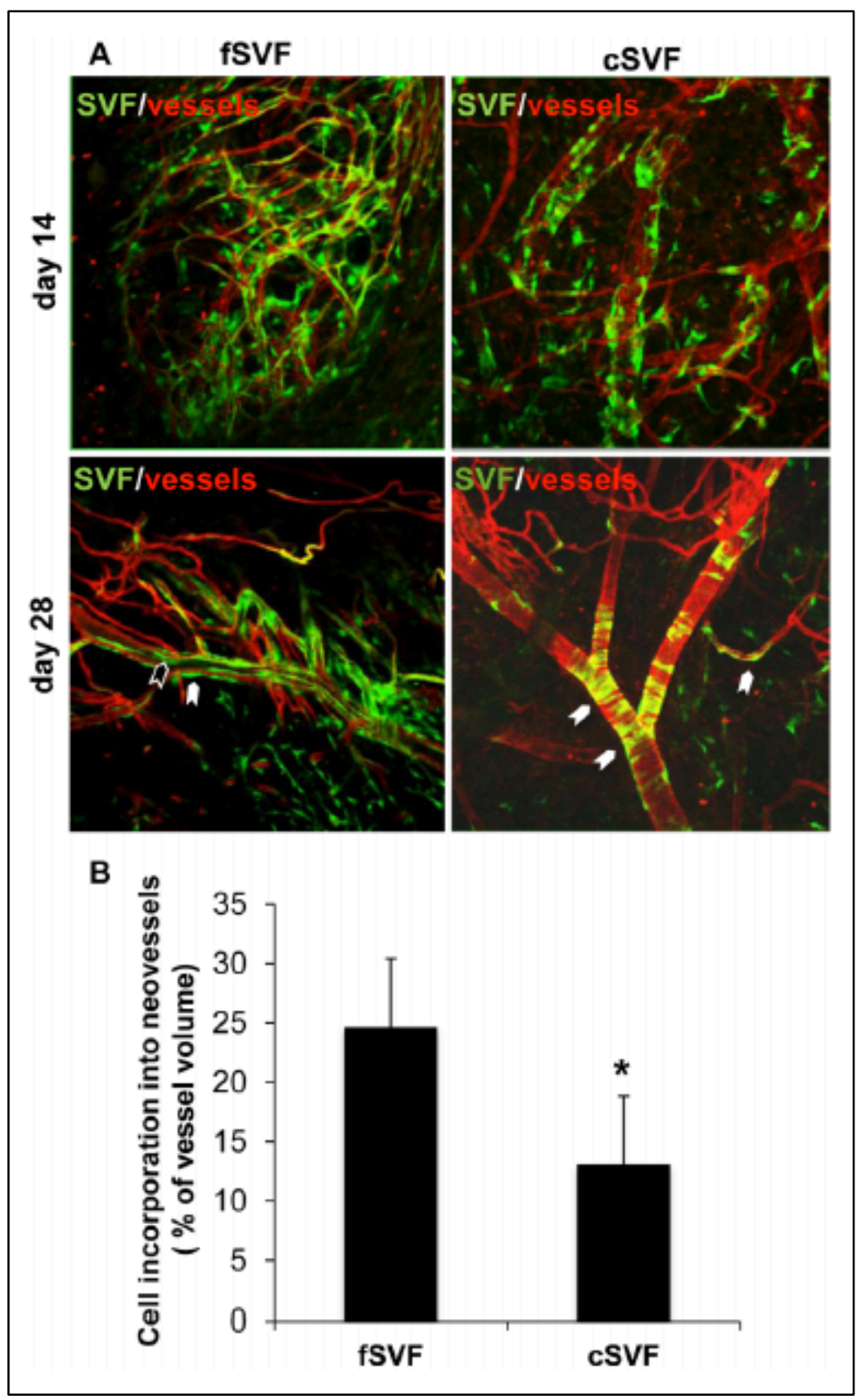

Figure 7. Fresh and cultured SVF cells differ in their ability to participate in angiogenesis. GFP-laden $3 D$ collagen constructs were implanted in non-GFP mice for 14 or $28 d$. The images and quantitation demonstrate that significantly fewer cultured SVF cells incorporate into developing vessels $\left({ }^{*} p=0.01\right)$ and that most cultured SVF cells take perivascular positions. Results shown as mean \pm S.E.M. 
What is most unique about these two populations is that fSVF yielded vessels in which $\mathrm{GFP}^{+}$cells were part of both the main vascular tube as well as perivascularly. To contrast, cSVF cells predominantly occupied the perivascular space. Along the same lines, fewer cSVF cells were incorporated into the neovascular network $(13 \pm 6.6 \%$, vs. $24.6 \pm 10.4 \%$ for $\mathrm{fSVF})$.

\section{Cultured rodent SVF has fewer endothelial and progenitor cells than fresh}

rodent SVF. Given that we saw numerous quantifiable differences between cSVF and fSVF, we next wanted to determine if there were differences in the cellular composition of these populations that drove their respective phenotypes. Using flow cytometry, cSVF were observed to contain roughly $50 \%$ less $\mathrm{CD}^{+} 1^{+}$(presumably endothelial) cells than fSVF. Moreover, a similar trend was seen with respect to the number of cKit progenitor

cells (Figure 8). Despite these differences, the proportions of CD14 $4^{+}$monocytes and macrophages, PDGF-R $\beta^{+}$perivascular cells, and CXCR4 and $\mathrm{cMet}^{+}$multipotent cells remained approximately the same. Thus, the number of endothelial and progenitor cells in SVF may be diminished with culturing, while perivascular presence and functionality appear to be unaffected.

Implanted parenchyma can be vascularized by human SVF cells. Thus far, cultured and fresh SVF cells have been shown to possess a unique vascularization potential. We therefore wanted to assess the role of these cells in the presence of an implanted parenchymal cell model - in this case, HepG2 model hepatocytes (Figure 9). Here, we used human SVF as the HepG2 were also derived from a human source and we 


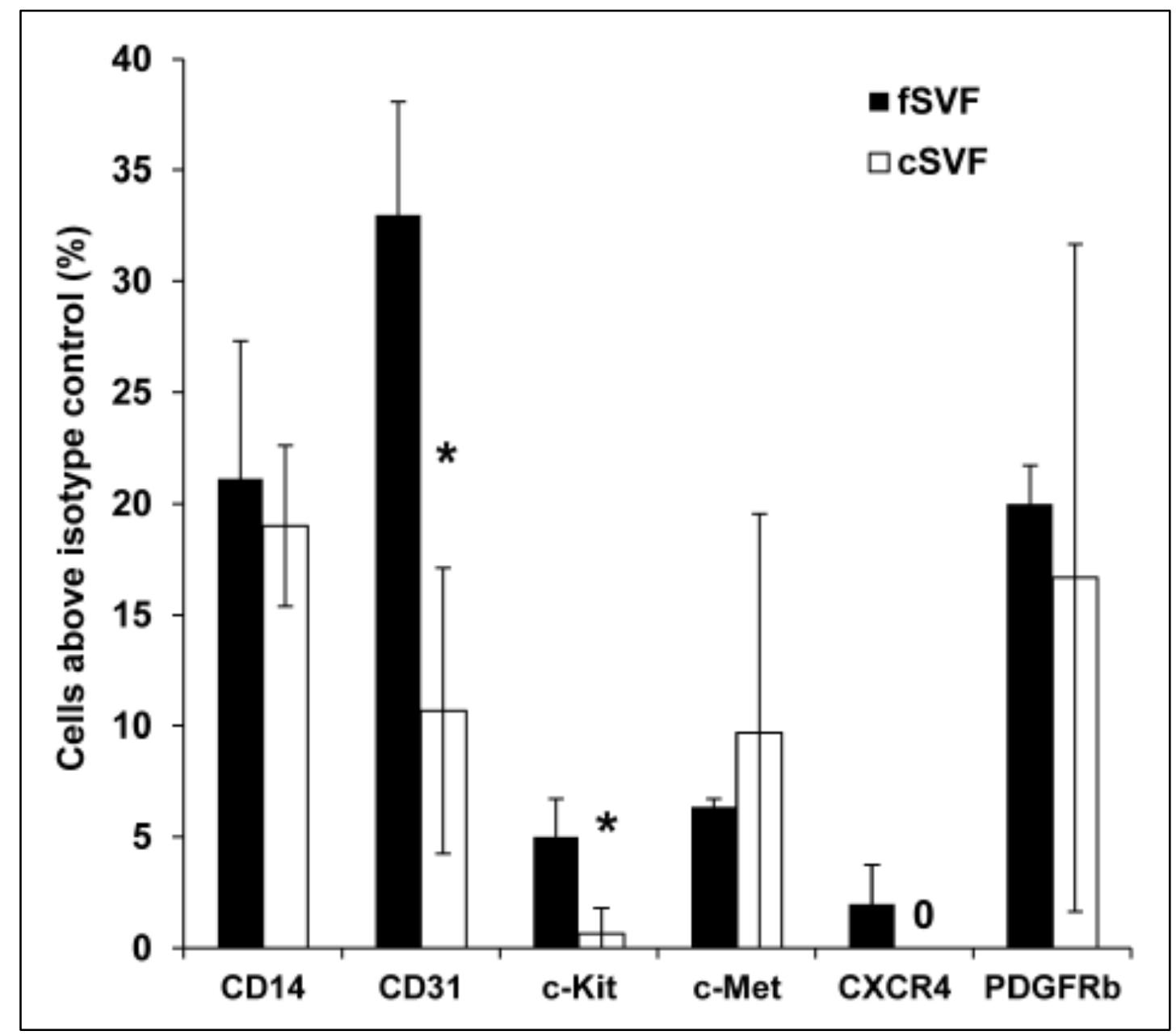

Figure 8. Flow cytometry performed on cultured versus fresh SVF cells show compositional differences between the two cell populations, the most significant of which are reductions in $C D-31^{+}$endothelial cells $\left({ }^{*} p=0.009\right)$ and $c \mathrm{Kit}^{+}$progenitor cells $\left({ }^{*} p=\right.$ 0.02) in cultured SVF. Results shown as mean \pm S.E.M. 


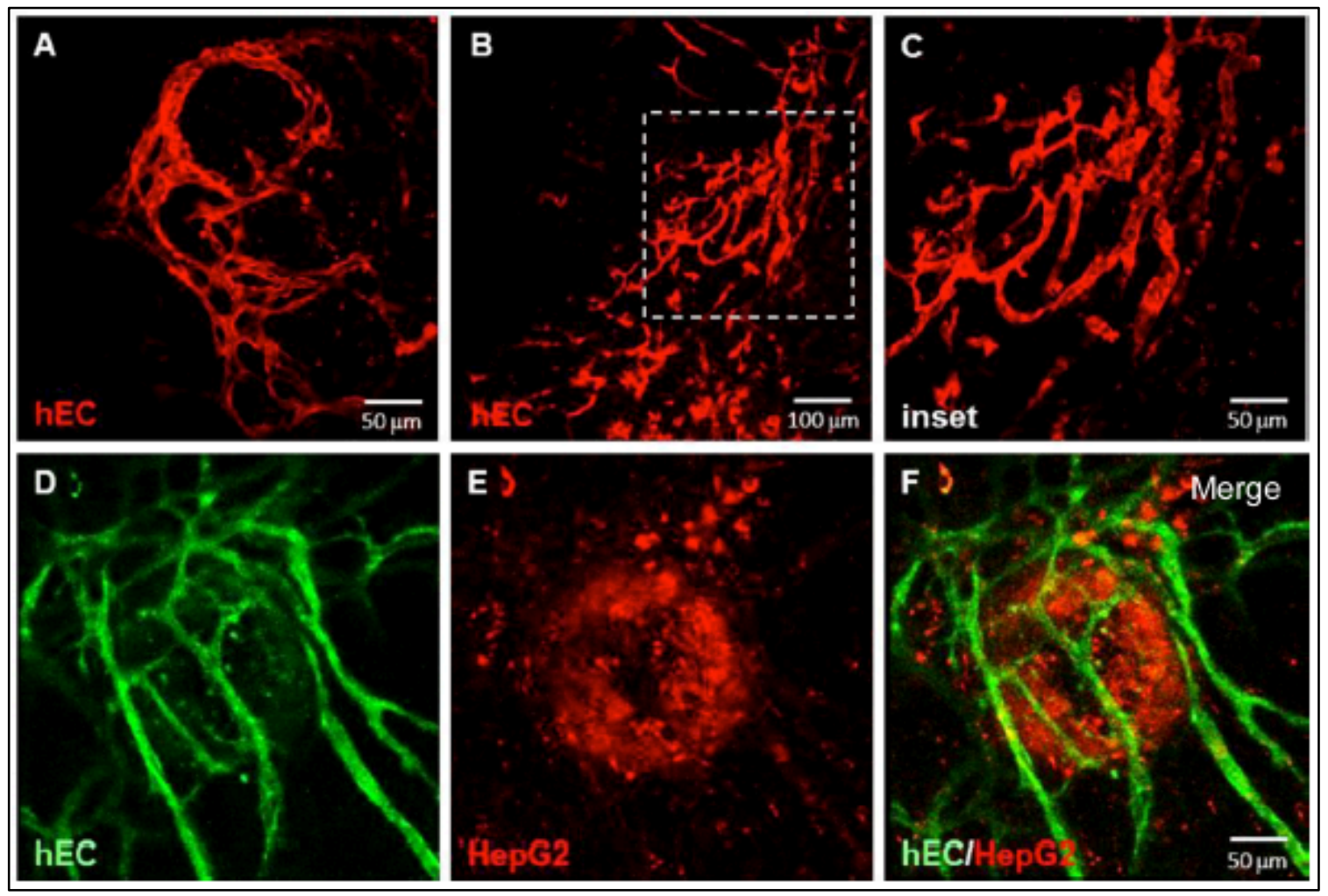

Figure 9. Figures A-C show human SVF within $3 D$ collagen I gels that were implanted for 4 weeks, explanted, and labeled with UEA-1 TRITC. Figures D-F show that at 6 weeks, human SVF form as vascular cage around implanted HepG2 model hepatocyte spheroids. 
sought to create a completely human mimic. Thus, because these SVF cells were isolated from discarded human lipoaspirate instead of from rat fat pads, we opted for a 6-week implant time instead of a 4-week implant time. This would allow us to begin accounting for potential species-dependent differences in network formation.

Our first observation was that indeed, like rodent SVF, human SVF was also capable of spontaneously self-assembling into a vascular network. However, at 6 weeks, it is possible that human SVF cells required even more time to reach the same degree of maturation as the rodent-derived SVF vascular networks seen at 4 weeks. Secondly, we wished to see if human SVF could spontaneously form a vascular network in the presence of parenchymal cells. To this end, we generated 3D constructs containing HepG2 (grown on cytodex beads to facilitate hepatocyte survival and function) and human SVF cells. Here too, human SVF spontaneously formed a vascular network. However, in this particular arrangement, human SVF networks could also be seen forming cage-like structures around HepG2 clusters (Figure 9).

\section{Human fresh SVF-derived vasculatures inosculate implanted parenchymal}

cells. Since we observed human SVF-derived vascular networks surrounding HepG2 clusters, we next wanted to determine the degree of association between the vasculature and the parenchyma. Because HepG2 can bind and internalize LDL cholesterol similar to primary hepatocytes, this provided us with a suitable assay to assess SVF-vascular inosculation of the parenchyma.

After injecting fluorescent DiI-LDL cholesterol intravenously and allowing for sufficient circulation time, we observed the accumulation of DiI-LDL within implanted 
HepG2 cells (Figure 10). Conversely, 3D constructs lacking SVF (but containing HepG2) were not able to localize DiI-LDL. To compliment these findings, image analysis revealed that in $3 \mathrm{D}$ constructs containing both cell types, $83 \%$ of HepG2 clusters were associated with either vasculatures or DiI-LDL internalization, while $67 \%$ were associated with both.

Thus, these findings indicate that human SVF cells form a vascular network that facilitates, and perhaps even enables, the functionality of parenchymal implants. 


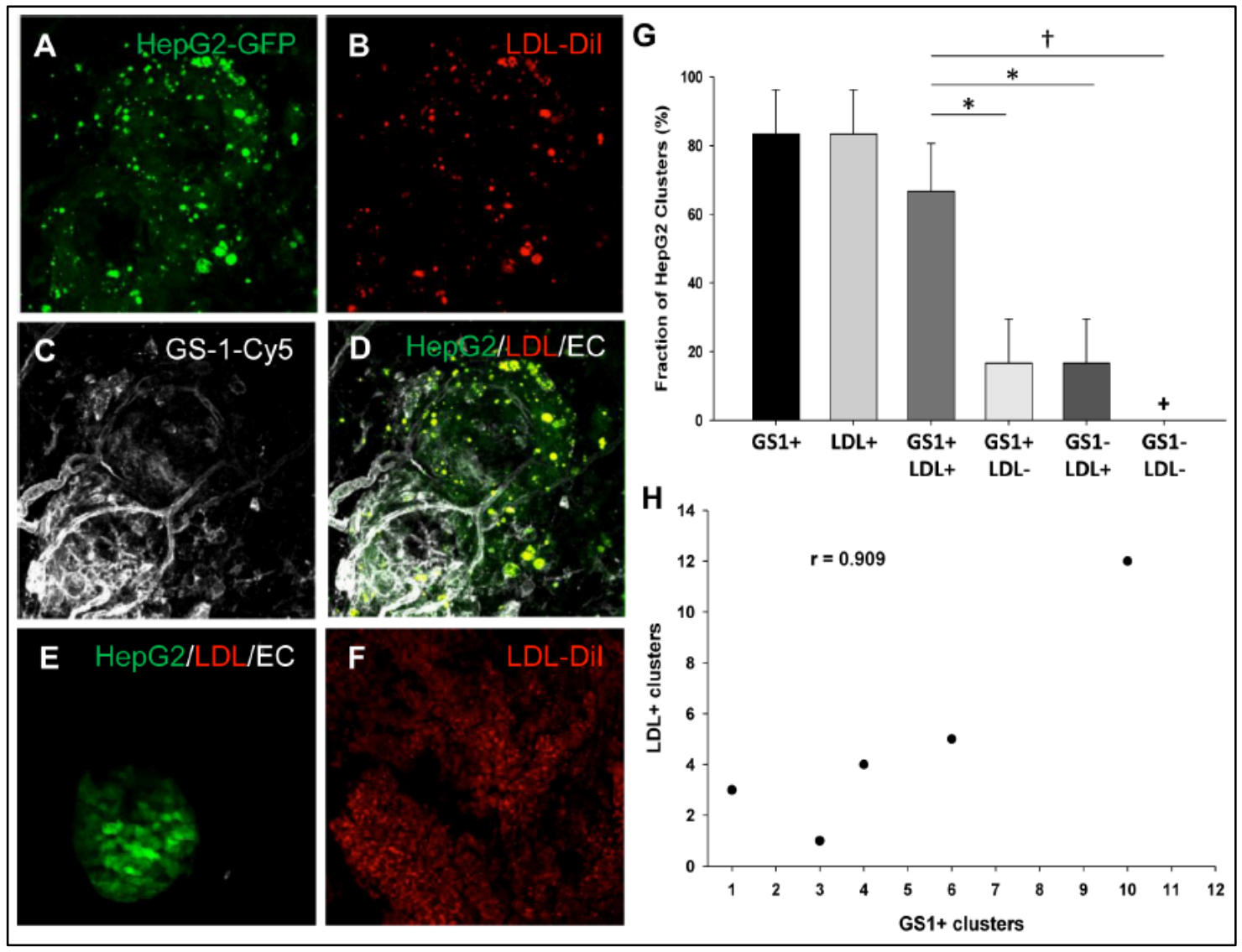

Figure 10. Figures A-D show confocal microscopy images of $G F P^{+} H e p G 2$, injected DiI$L D L$, and rodent SVF (labeled with GS1-Cy5) all co-localizing. Figures E-F demonstrate that without SVF, peripherally injected DiI-LDL does not accumulate in implanted HepG2 cells (E), though it does accumulate in the host liver $(F)$. Figure G quantifies the association between $\mathrm{GFP}^{+} \mathrm{HepG}, \mathrm{GS1}^{+} \mathrm{SVF}$, and DiI-LDL. Note that in HepG2-SVF implants, the majority of HepG2 are associated with LDL localization and SVF-derived endothelial cells $\left({ }^{*} p=0.03,{ }^{\dagger} p=0.08\right)$. (H) This scatter plot demonstrates that in HepG2-loaded implants, an increase in $G S 1^{+}$SVF clusters drives corresponds with an increased incidence of $L D L$ co-localization in HepG2. 


\section{DISCUSSION}

The next stage of our effort involves combining parenchymal cells with a vascular supply. As mentioned earlier, the development of a stable vasculature is critical to the development of large volumes of engineered tissues. Because our goal is to ultimately create a liver-tissue mimic that serves as a therapy for those with Familial Hypercholesterolemia, we developed a proof-of-concept by combining HepG2 model hepatocyte cells with adipose-derived stromal vascular fraction (SVF) cells. With this combination, we were the first to generate a functional, vascularized liver-tissue mimic model.

To date, it has been difficult to engineer mature vascular systems capable of supporting tissues and organs ${ }^{353,354}$. Several have attempted to use endothelial cells alone, but several have observed that multicellular, three-dimensional approaches are required to form mature vasculatures ${ }^{355-357}$. To this end, perivascular cells are considered critical constituents of the developing vasculature ${ }^{321,358}$. SVF cells show tremendous therapeutic potential, as they are a heterogeneous mixture of various cell types that can be applied in a variety of circumstances. The cells can be acquired relatively easily in lipoaspirates, obtained through minimally invasive means and even at the patient's bedside. While this means that the cells can be acquired and used at the point of care ${ }^{333}$ (Tissue Genesis Clinical Trial \# NCT02234778), an added benefit is that the patient's own cells can be used. This allows the entire process to remain autologous, minimizing the chance of rejection.

Our findings indicate that cultured SVF can form perfused vasculatures in vivo as well. Thus, with regards to clinical applicability, a pooled, cultured supply of SVF cells 
acquired from various donors could provide added convenience and potentially mitigate deficiencies present within any one isolate. Though one hurdle with this approach is that only cells from a low passage number can be utilized, one benefit here is that adipose cells are limited in their ability to incite a host immune response ${ }^{302,359}$. Therefore, while an autologous approach may be desirable, SVF allografts may also be clinically acceptable by providing a workaround under non-ideal circumstances. Additionally, the use of allografted SVF may facilitate the use of allogenic parenchymal cells $\mathrm{s}^{205,360}$

In this light, adipose-derived SVF cells constitute a vital part of the liver-tissue mimic. While previous attempts at vascularizing engineered liver grafts involved incorporating VEGF into constructs containing transplanted hepatocytes, it is unknown if any resulting vessels were perfused, or if those vessels enabled or enhanced hepatocyte functionality ${ }^{361}$. To contrast, freshly isolated, cultured and frozen SVF cells can all spontaneously form mature, hierarchical vasculatures. Furthermore, SVF cells have been used in Phase I clinical trials and are considered to be very safe ${ }^{362}$.

To demonstrate SVF cell functionality in the context of a liver-tissue mimic, mimics with and without SVF were implanted for six weeks. Just prior to explantation, host animals were given fluorescent DiI-LDL cholesterol to assess SVF's role in forming a functional interface between implanted HepG2 cells and the host vasculature. Our findings demonstrate that HepG2 cells within constructs containing SVF were able to internalize circulating DiI-LDL cholesterol. To contrast, mimics without SVF were not able to internalize DiI-LDL. These findings indicate that the SVF cells serve in a key capacity by functionalizing co-implanted parenchymal cells. Additionally, SVF-derived vessels have lumens, anastomose with the host vasculature, are perfused, and thus serve 
as a conduit for circulating macromolecules. All of this suggests that SVF cells could be combined with parenchymal cells to enable and facilitate parenchymal metabolism of various biomolecules.

Our liver-tissue mimic (comprised of parenchymal and SVF cells) was designed as a modular implant. This poses several potential advantages. First, let us consider the impact of a single module. In this case, our liver-tissue mimic would be responsible for LDL cholesterol uptake, but in other circumstances, such modules could be used for hormone production or drug clearance. Benefits of this approach are that (a) modules are easily scalable, and (b) their arrangement can be customized. For instance, modules of different compositions could be combined together to perform a whole host of functions. Second, a module like this could be implanted anywhere there is access to a host blood supply. The placement of a modular scaffold subcutaneously has the added benefit of being easily accessible, modifiable, and in deleterious circumstances, removable. With respect to FH, a modular liver-tissue mimic could be ideal. While FH patients have impaired LDLR function, their other liver functions are normal. Though curative from an LDLR standpoint, performing a full liver transplant could be heavy-handed, as transplant-associated complications may impair functions that were once normal. Therefore, an organ-mimic module may enable a targeted approach to treating a specific deficiency. Third, modules such as these could serve as drug toxicity testing platforms for testing drug effects on various parenchymal phenotypes.

Cultured SVF cells can spontaneously form vasculatures at low passages, as mentioned earlier, though there are a few caveats. First, compared to cells that were freshly isolated, the proportion of cultured SVF cells that incorporate into neovessels is 
decreased. Secondly, the positioning of these incorporated cells is different, as fresh isolates take on vascular and mural positions while cultured cells are predominantly located in the perivascular space. Third, culturing the cells decreases the quantity of $\mathrm{CD} 31^{+}$and $\mathrm{cKit}^{+}$cells, suggesting that endothelial cell presence is diminished by culturing $^{363-365}$. Because SVF-driven vasculogenesis is endothelial cell-driven ${ }^{299}$, and cultured SVF has fewer endothelial cells, cultured SVF possesses a reduced (but not nonexistent) ability to form vasculatures in vivo. Therefore, culturing impairs SVF cells' ability to participate in neovascularization.

While culturing reduces the number of endothelial cells in SVF, it appears to have no effect on the amount of PDGFR- $\beta 1^{+}$perivascular cells ${ }^{366,367}$. This finding corresponds with the observation that cultured SVF cells predominantly reside in mural positions. Since culturing clearly effects SVF cell dynamics, it is important to acknowledge that our SVF culture methods differ from those used by others to select for adipose-derived stem cells (ADSC) $)^{298,368}$. Our low-passage cultured SVF expresses markers associated with several mesenchymal stem cell phenotypes. We also have other phenotypes that do not appear to be present in the ADSC populations that others have isolated. Therefore, the presence of unique phenotypes in our cultured isolates may dictate SVF's ability to spontaneously assemble into a functional neovasculature, though this ability is impaired compared to SVF cells from fresh isolates. Additionally, the effect of long-term culture (beyond P1) on vascular network formation is a subject for further investigation (and is discussed in Chapter 3).

Again, SVF is unique because of its ability to spontaneously assemble into a mature, hierarchical, perfused, functional vascular system in vivo. Koh demonstrated that 
this phenomenon was dependent on endothelial cells, and that vascular network formation was abrogated in the absence of endothelial cells ${ }^{299}$. Yet, endothelial cells alone are unable to form as dynamic a vasculature, with the aforementioned characteristics, in vitro ${ }^{316}$ or in vivo ${ }^{317}$. Therefore, perivascular cells appear to play a critical role in shaping, maturing, stabilizing, and functionalizing an SVF-derived developing vasculature ${ }^{316,318}$.

While several tissues possess complex vasculatures, isolating and utilizing vessels from these sources does not guarantee the same level of performance as that seen with SVF cells. This is likely because SVF is derived from adipose tissue, which is thought to have a less mature vasculature with a high degree of plasticity ${ }^{321}$. Such plasticity may be required to meet adipose tissue's complex metabolic needs ${ }^{321}$.

This is the first effort that demonstrates that human and rodent adipose-derived SVF cells can inosculate with the host vasculature and vascularize implanted parenchyma (in this case, HepG2 hepatocyte model cells), to form a proof-of-principle organ-tissue mimic. This mimic poses several implications for a variety of organs and disease states. The nature of its design allows different parenchymal cells to be used to meet different needs. With respect to Familial Hypercholesterolemia, a disease of LDLR dysfunction, our liver-tissue mimic enabled the uptake of injected, circulating LDL cholesterol by hepatocytes within the mimic. This suggests that SVF-derived vessels can help impart critical functionality to implanted hepatocytes, and allows us to take a critical step towards developing a therapeutic device for this patient population. This mimic also opens the doors for further mechanistic and translational investigations. 
CHAPTER IV

WNT5A MODULATES THE SELF-ASSEMBLY OF HUMAN STROMAL VASCULAR FRACTION-DERIVED MICROVASCULATURES

\section{OVERVIEW}

Human adipose-derived stromal vascular fraction (hSVF) cells are an easily accessible, heterogeneous cell system that can spontaneously self-assemble into functional microvasculatures in vivo. However, the mechanisms underlying this vascular self-assembly and maturation remain poorly understood. Thus, our objective was to identify regulatory pathways mediating this endogenous process. It has been shown that culture of SVF reduces its vascularization capacity. Here, we found that EC network densities were significantly diminished as early as two passages (P2) and was almost eliminated by the fourth passage (P4). For investigating vascularization mechanisms, subsequent experiments only used P1 cells. After exposing hSVF cells to common angiogenesis inhibitors, we found that the pan-Wnt inhibitor IWP2 produced the most significant networking decrease $(\sim 25 \%$ reduction), implicating Wnt signaling in this phenomenon. To determine which Wnt isoform(s) and receptor(s) may be involved, hSVF was screened by PCR for those isoforms associated with angiogenesis. Of these, only WNT5A and its receptor, FZD-4, were stably expressed. Immunocytochemistry showed that Wnt5a protein was expressed in hSVF cultures. To see if Wnt5a alone could 
restore IWP2-induced EC network inhibition, recombinant human Wnt5a (0-150 ng/ml) was added to IWP2-treated cultures. Addition of rhWnt5a to such cultures significantly increased EC network area and significantly decreased the ratio of total EC network length to EC network area compared to untreated controls. To determine if Wnt5a mediates in vivo microvascular self-assembly, $3 \mathrm{D}$ hSVF constructs containing an IgG isotype control, anti-Wnt5a neutralizing antibody or rhWnt5a were implanted subcutaneously for two weeks. Compared to $\operatorname{IgG}$ controls, anti-Wnt5a treatment significantly reduced vessel length density by $\sim 41 \%$, while rhWnt5a significantly increased vessel length density by $\sim 62 \%$. However, anti-Wnt5a or rhWnt5a did not affect the density of segments and nodes, both of which measure vascular complexity. Taken together, this data demonstrates that hSVF microvascular self-assembly is mediated by Wnt5a. These findings also suggest that manipulating Wnt signaling could enhance control of hSVF vascularization in tissue engineering applications.

\section{INTRODUCTION}

The vascularization of engineered tissues remains a significant challenge in tissue engineering. Adipose-derived stromal vascular fraction (SVF) is a heterogeneous cell population $314,319,344,369$ that has been shown to spontaneously self-assemble into functional, hierarchical, and perfused vasculatures in vivo $9,299,370$ and vessel-like networks in culture ${ }^{371,372}$. These findings suggest that SVF may have potential applications toward the accelerated formation of new microvascular structures in therapeutic applications. Our lab has previously demonstrated that SVF can accelerate the vascularization and engraftment of co-implanted model hepatocyte cells ${ }^{9}$ and induced 
pluripotent stem cell-derived hepatocytes ${ }^{1}$. Spontaneous self-assembly into microvasculatures by the SVF cells requires the presence of endothelial cells (EC) within the isolate, as their removal eliminates the vascular assembly activity ${ }^{9,299}$. Yet, the mechanism(s) by which SVF EC assemble into a functional microvasculature is not well defined.

To date, vascular assembly in SVF has been described as being VEGFdependent ${ }^{299}$, but experience with other systems suggests that multiple signaling pathways mediate the final microvascular system $316,344,345,373-375$. To better understand how human SVF (hSVF) cells self-assemble a microvasculature, we investigated several angiogenesis-related signaling pathways to determine their role. Here, we used an in vitro model to identify Wnt5a as a potential mediator of vascular assembly. We demonstrate that Wnt5a affects parameters such as vessel length and area coverage - effects that were absent when hSVF was supplemented with canonical Wnt3a in lieu of Wnt5a. In threedimensional in vivo implants, the treatment of hSVF with a Wnt5a-specific neutralizing antibody significantly reduced hSVF EC network assembly as quantified by total EC length density compared to IgG controls. In contrast, the addition of recombinant human Wnt5a significantly increased the total EC length density. Both anti-Wnt5a and recombinant human Wnt5a did not significantly affect the densities of segments and nodes, suggesting that Wnt5a alone may not modulate vascular complexity. To our knowledge, this is the first report to characterize the role of Wnt5a in spontaneous hSVF EC microvascular self-assembly. Our data suggests that Wnt5a is a potential target for therapeutic angiogenic modulation to regulate hSVF vascularization. 


\section{MATERIALS \& METHODS}

Animals and Ethics Statement. All animal procedures were conducted in compliance with University of Louisville School of Medicine IACUC-approved protocols and NIH guidelines. Isoflurane gas was administered for anesthesia. For analgesia, Ketoprofen was delivered subcutaneously at the time of surgery and postoperatively every $24 \mathrm{~h}$ for $2 \mathrm{~d}$.

hSVF Isolation, Long-Term Storage, and General Culture. hSVF cells were isolated from the discarded lipoaspirate of three patients (identifying information was unavailable) obtained under IRB exemption \#09.0037. The lipoaspirate was acquired and processed as previously described ${ }^{9,370}$. The resulting cell pellet was divided and stored in liquid nitrogen. Thawed cells were resuspended in hSVF growth media (M199, 1X Lglutamine, and HEPES (Invitrogen), 10\% FBS (VWR International), and EC growth supplement (developed in-house and augmented with heparin)) and plated into a $75 \mathrm{~cm}^{2}$ NUNC tissue culture flask (Thermo-Fisher Scientific) pre-coated with $1 \%$ gelatin (Sigma-Aldrich). Cells were cultured overnight at $37^{\circ} \mathrm{C}$ and $5 \% \mathrm{CO}_{2}$. A media change was performed the following morning to remove non-adherent cells, with subsequent media changes performed every other day. Once confluent, hSVF cells were passaged with $0.05 \%$ Trypsin-EDTA (Invitrogen).

In Vitro Network Assembly. hSVF cells from passages 1 through 4 (P1 - P4) were plated at a density of $6 \times 10^{4}$ cells $/ \mathrm{cm}^{2}$. At days 1,3 , and 5 post-plating, all supernatant was aspirated and the cells were fixed in $1 \%$ paraformaldehyde (PFA; Electron Microscopy Sciences) for 10 minutes at RT before being washed with PBS (Invitrogen) for 5 minutes. All wells were labeled with Ulex Europaeus Agglutinin-1 
(UEA1; Vector Laboratories) for human EC as well as DAPI nuclear dye (Thermo Fisher). Cells were imaged using an IX81 inverted fluorescence microscope (Olympus).

Inhibitor Treatment. P1 hSVF cells were plated as described and, the following day, subjected to treatment with the Wnt release inhibitor IWP2 (Tocris Biosciences) (at $0,6.25,12.5$, and $25 \mu \mathrm{M}$; also see Table 1 and Figure 11 for a listing and the effects, respectively, of the other tested angiogenic inhibitors) for 5 days $^{376}$. Media changes were performed every other day. At the end of day 5, the cells were processed and imaged as described above.

Cytotoxicity Assay. hSVF cells were cultured in different IWP2 concentrations $(0,6.25,12.5,25 \mu \mathrm{M}$, and $50 \mu \mathrm{M})$ and assayed for cytotoxicity using CCK-8 (Dojindo Molecular Technologies) according to the manufacturer's instructions. We measured absorbance using a Synergy 4 plate reader (BioTek).

Gene and Protein Expression. Samples from P1 hSVF cell cultures were acquired on days 1, 3, and 5 and processed for mRNA isolation using QIAShredder and RNeasy kits (Qiagen) according to the manufacturer's instructions. cDNA production, RT-PCR, and gel electrophoresis were all conducted as previously described ${ }^{1}$. RT-PCR reactions (primers can be found in Table 2) utilized the following settings: 25 cycles, with denaturation at $95^{\circ} \mathrm{C}$, annealing at $59^{\circ} \mathrm{C}$, and extension at $70^{\circ} \mathrm{C}$ for 30 seconds. Detected bands were normalized to GAPDH via densitometry analysis with Image $\mathrm{J}$ software (NIH). For analysis of protein expression, wells were prepared for fluorescence microscopy as previously described ${ }^{1}$. Cells were permeabilized in $0.5 \%$ Triton X-100 (MP Biomedicals) for 10 minutes, blocked in 5\% goat serum (Thermo Fisher) for 1 hour, and incubated with anti-human Wnt5a antibody (Abcam) at 1:250 and $4^{\circ} \mathrm{C}$ overnight. 


\begin{tabular}{|c|c|c|c|c|}
\hline Inhibitor & Designation & $\begin{array}{c}\text { Vendor (Catalog } \\
\text { No). }\end{array}$ & Dose Range & $\begin{array}{c}\text { Significant } \\
\text { Effect } \\
\text { Observed? }\end{array}$ \\
\hline $\begin{array}{c}\text { Wnt } \\
\text { Palmitoylation }\end{array}$ & IWP2 & Tocris (3533) & $\begin{array}{c}0,6.25,12.5, \\
25, \text { and } 50 \\
\mu \mathrm{M}\end{array}$ & Yes \\
\hline VEGF-R2 & ZM323881 & Tocris (2475) & $\begin{array}{c}0,0.5,2.5,5 \\
\text { and } 10 \mu \mathrm{M}\end{array}$ & Yes \\
\hline PDGF-R $\beta$ & AG1296 & $\begin{array}{c}\text { Calbiochem } \\
\text { (658551) }\end{array}$ & $\begin{array}{c}0,0.5,5,25, \\
\text { and } 50 \mu \mathrm{M}\end{array}$ & Yes \\
\hline TGF-ß1 / Alk-5 & SB431542 & $\begin{array}{c}\text { Stemcell } \\
\text { Technologies } \\
(72232)\end{array}$ & $\begin{array}{l}0,0.5,1,5 \\
\text { and } 10 \mu \mathrm{M}\end{array}$ & No \\
\hline HGF / cMet & SU11274 & $\begin{array}{c}\text { Selleck Chemicals } \\
\text { (S1080) }\end{array}$ & $\begin{array}{l}0,0.5,1,5 \\
\text { and } 10 \mu \mathrm{M}\end{array}$ & No \\
\hline
\end{tabular}

Table 1. List of common angiogenic inhibitors used to isolate potential mechanisms of hSVF vascular self-assembly. 


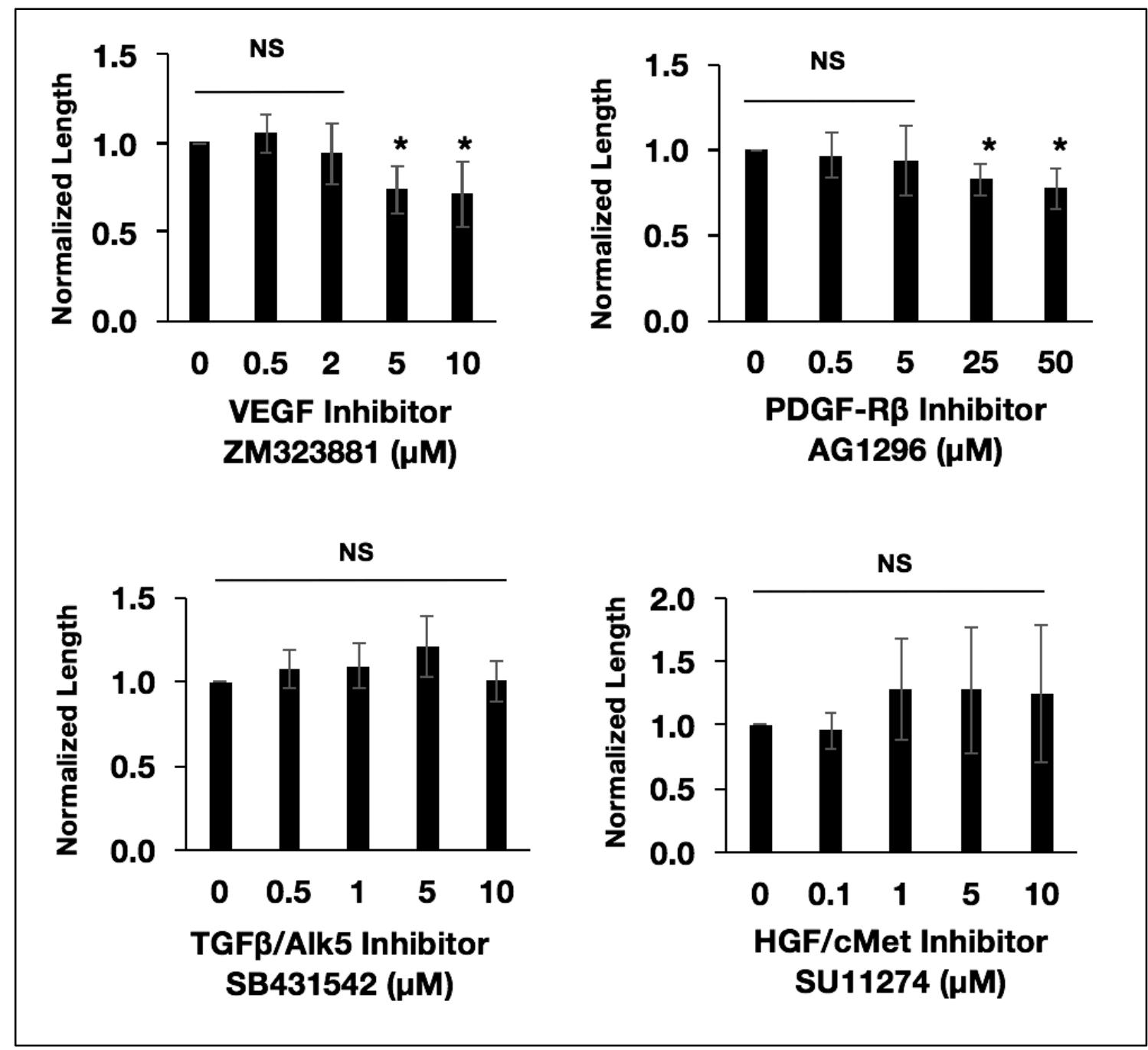

Figure 11. hSVF EC network quantification after inhibitor treatment. $h S V F$ was treated with small molecule inhibitors to identify potential pathways mediating vascular self-assembly. For each inhibitor tested, $U E A 1^{+}$network length was normalized to the untreated control (also see Table 4). The VEGF-R2 and PDGF-R $\beta$ inhibitors significantly reduced total hSVF EC network length at their two highest concentrations $\left({ }^{*} p \leq 0.05\right)$, whereas no significant differences were seen with any concentration of the TGF-ß/Alk5 or HGF/cMet inhibitors. Results shown as mean \pm S.E.M. 


\begin{tabular}{|c|c|c|c|c|c|}
\hline Gene & $\begin{array}{c}\text { NCBI } \\
\text { Reference } \\
\text { Sequence }\end{array}$ & $\begin{array}{l}\text { 5' } \rightarrow \text { 3' Primer Sequence } \\
\text { (Forward / Reverse) }\end{array}$ & $\begin{array}{l}\text { Size } \\
\text { (bp) }\end{array}$ & $\begin{array}{c}\text { Annealing } \\
\text { Temp. }{ }^{\circ} \mathrm{C} \\
\text { (Forward / } \\
\text { Reverse) } \\
\end{array}$ & $\begin{array}{l}\text { Expressed } \\
\text { in hSVF? }\end{array}$ \\
\hline WNT2 & NM_003391.2 & $\begin{array}{l}\text { CTGGCCTTTATCGCTCGCTG / } \\
\text { TTCATGACCACCTGGATGG }\end{array}$ & 943 & $61.49 / 60.03$ & No \\
\hline WNT3A & NM_033131.3 & $\begin{array}{l}\text { CCTCCCTGGAGCTAGTGTCT / } \\
\text { GGAACCTTACAGGGGGTTGG }\end{array}$ & 632 & $60.03 / 59.96$ & No \\
\hline WNT4 & NM_030761.4 & $\begin{array}{l}\text { TCGTCTTCGCCGTCTTCTCAG / } \\
\text { GAGTCGAGTGTGGAGCAGTT }\end{array}$ & 244 & $62.12 / 59.68$ & No \\
\hline WNT5A & NM_003392.4 & $\begin{array}{c}\text { AAGCAGACGTTTCGGCTACA / } \\
\text { TTTCCAACGTCCATCAGCGA } \\
\end{array}$ & 287 & $59.97 / 59.97$ & Yes \\
\hline WNT5B & NM_030775.2 & $\begin{array}{l}\text { CTCAAGAGAGCGAGAAGACTGG / } \\
\text { CССТCCССTATGTAGGCCAT }\end{array}$ & 309 & $60.16 / 60.18$ & No \\
\hline WNT7B & NM_058238.2 & $\begin{array}{l}\text { GGAGGCTTCCACCTTTCTCC / } \\
\text { TCTCCGGTACCCAGTGTAGG }\end{array}$ & 715 & $60.03 / 60.03$ & Yes \\
\hline WNT10B & NM_003394.3 & $\begin{array}{l}\text { TGAGCTCGGTGAGAGCAAAG / } \\
\text { TTAAACCGTGGGGAGACTGC }\end{array}$ & 204 & $60.04 / 59.96$ & No \\
\hline WNT11 & NM_004626.2 & $\begin{array}{l}\text { CACAAGACAGGCAGTGCAAC / } \\
\text { TGCCGAGTTCACTTGACGAG }\end{array}$ & 883 & $59.97 / 60.32$ & No \\
\hline FZD4 & NM_012193.3 & $\begin{array}{l}\text { CCAACTGGGCACTTTTTCGG / } \\
\text { TCTAAACAGCAGACAGCGCA }\end{array}$ & 783 & $59.97 / 59.97$ & No \\
\hline FZD5 & NM_003468.3 & $\begin{array}{l}\text { TGCTTCATCTCCACGTCCAC / } \\
\text { AGGATGACCCACCAGATGGA }\end{array}$ & 272 & $60.04 / 59.96$ & No \\
\hline ROR2 & NM_004560.3 & $\begin{array}{l}\text { TCCTTCTGCCACTTCGTCTT / } \\
\text { TTGTAGCACTGGTGGTAGCG }\end{array}$ & 266 & $58.95 / 60.04$ & No \\
\hline GAPDH & NM_001289745.1 & $\begin{array}{l}\text { AATCCCATCACCATCTTCC / } \\
\text { CATCACGCCACAGTTTCC }\end{array}$ & 382 & $54.14 / 56.43$ & No \\
\hline
\end{tabular}

Table 2. List of primers used in RT-PCR with hSVF cDNA. 
Goat anti-Rabbit 594 secondary antibody (Thermo Fisher) was added the following day at 1:1000 for 1 hour at RT (refer to Table 3 for further details). Wnt5a was visualized using an IX81 inverted microscope (Olympus) and MPE FV1000 confocal microscope (Olympus).

Exogenous Wnt5a Treatment. After plating overnight in hSVF Growth Media (GM), hSVF cells were treated with $25 \mu \mathrm{M}$ IWP2 and varying concentrations of recombinant Wnt5a $(0,0.75,7.5,75$, and $150 \mathrm{ng} / \mathrm{ml}$; R\&D Systems) for 5 days. As a comparison, recombinant human Wnt3a was added to $25 \mu \mathrm{M}$ IWP2 in hSVF GM at the same concentrations. Media changes were performed every other day. At the end of day 5, all of the wells were labeled with UEA1 and quantified as previously described. 


\begin{tabular}{|c|c|c|}
\hline $\begin{array}{l}\text { Antibody/Lectin/Stain } \\
\text { (Concentration) }\end{array}$ & $\begin{array}{c}\text { Manufacturer } \\
\text { (Catalog Number) }\end{array}$ & Purpose \\
\hline Wnt5a $(1: 250)$ & Abcam (ab72583) & Immunocytochemistry \\
\hline Wnt5a $(20 \mu \mathrm{g} / \mathrm{ml})$ & R\&D Systems (AF-645) & Neutralization \\
\hline $\operatorname{IgG}(20 \mu \mathrm{g} / \mathrm{ml})$ & R\&D Systems (AB-108-C) & Control \\
\hline $\begin{array}{c}\text { Fluorescein-Labeled Ulex } \\
\text { Europaeus Agglutinin Type I } \\
\text { (UEA1; 1:500) }\end{array}$ & $\begin{array}{l}\text { Vector Laboratories (FL- } \\
1061)\end{array}$ & Immunocytochemistry \\
\hline DAPI $(1: 10000)$ & Thermo F & Immunocytochemistry \\
\hline $\begin{array}{c}2^{\circ} \text { Antibody } \\
\text { (Concentration) }\end{array}$ & $\begin{array}{c}\text { Manufacturer } \\
\text { (Catalog Number) }\end{array}$ & Purpose \\
\hline $\begin{array}{c}\text { Goat anti-Rabbit DyLight }{ }^{\mathrm{TM}} \\
594(1: 1000)\end{array}$ & $\begin{array}{c}\text { Thermo Fisher Scientific } \\
(35560)\end{array}$ & Immunocytochemistry \\
\hline
\end{tabular}

Table 3. List of the antibodies, lectins, and stains used. 
Wnt5a-Specific Neutralization and In Vivo Analysis. P1 hSVF cells were cultured as described in hSVF GM containing $0,5,10$, or $20 \mu \mathrm{g} / \mathrm{ml}$ of anti-Wnt5a antibody or Normal Goat IgG control antibody (R\&D Systems). Of note, though the antiWnt5a antibody used here is specific for mouse and rat, others have successfully used it to neutralize human Wnt5a as well ${ }^{377-379}$ Media changes occurred every other day. At the end of day 3, all of the wells were labeled with UEA1 and quantified as previously described.

To validate Wnt5a's role in vivo, hSVF was incorporated into $3 \mathrm{mg} / \mathrm{ml}$ collagen-I constructs as previously described ${ }^{9,370}$. One of three treatments was incorporated into each construct: (A) $20 \mu \mathrm{g} / \mathrm{ml} \mathrm{IgG} \mathrm{control} \mathrm{antibody,} \mathrm{(B)} 20 \mu \mathrm{g} / \mathrm{ml}$ anti-Wnt5a antibody, or (C) $7.5 \mathrm{ng} / \mathrm{ml}$ recombinant human Wnt5a. Constructs were placed bilaterally in the subcutaneous dorsum of $9 \mathrm{Rag1}^{-/-}$C57BL6 mice (Jackson Laboratories) as previously described $^{9,370}$. After 2 weeks, animals were sacrificed and the constructs explanted. Constructs were fixed in 4\% PFA for 1 hour, washed, permeabilized in $0.5 \%$ Triton X100 (MP Biomedicals) for 15 minutes, and blocked with 5\% goat serum (Thermo Fisher) for 1 hour. Constructs were incubated in 1:500 UEA1 overnight. The following day, they were washed and incubated with DAPI (1:10000) for 10 minutes. Imaging was conducted via fluorescence confocal microscopy (Olympus MPE FV1000).

2-D (In Vitro) Image Analysis. Fluorescence images were analyzed using Image J software (NIH) with the Neuron J plugin (ImageScience.org). Images were assessed for total EC network length and EC area. Sheets of ECs were not considered to be discrete segments and were omitted from total EC network length calculations, though they were incorporated into EC network area measurements. With regards to calculating the EC 
area, images were first given a color threshold to identify all UEA $1^{+}$structures. A size threshold of $0.065 \mathrm{~cm}^{2}$ was then applied to remove background noise. Resulting areas in a field-of-view were automatically quantified; images containing blatant outliers (for e.g., $\mathrm{UEAl}^{+}$particles not associated with a vascular structure) were manually excluded from EC area calculations. Regarding calculations and graphical representation, we normalized our in vitro rhWnt5a, rhWnt3a, and anti-Wnt5a quantitative data to their respective 0 $\mathrm{ng} / \mathrm{ml}$ or $0 \mu \mathrm{g} / \mathrm{ml}$ treatment controls. This was done to account for the inherent differences between three different hSVF isolations from three patients. Examples of length and area measurements are shown in Figure 12.

3D (In Vivo) Image Analysis. Z-stacks of confocal images were combined into a 3D composite image using AMIRA 6.0 software (FEI Visualization Sciences Group). After measuring $\mathrm{UEAl}^{+}$volume, we converted $\mathrm{UEA1}^{+}$signals into a skeleton and measured total EC network length, segments, and nodes. Network segments were considered to be structures with easily discernible origins and end points. The longest measurable vascular structure devoid of branching vessels was considered to be one segment. Each branch point was considered to be one node. All image manipulation and measurement was conducted based on instructions present in the AMIRA 6.0 User Guide.

Statistics. Experiments were conducted in triplicate. Error is presented as the mean \pm standard error of the mean (SEM). Significance was determined using the Student's $t$-test $(p<0.05)$. Overall significance of each treatment was determined by oneway ANOVA with follow-up Holm-Sidak or Tukey comparison tests, as determined by SigmaPlot (Systat Software). Outliers were identified using Chauvinet's criterion ${ }^{380}$ and removed. 


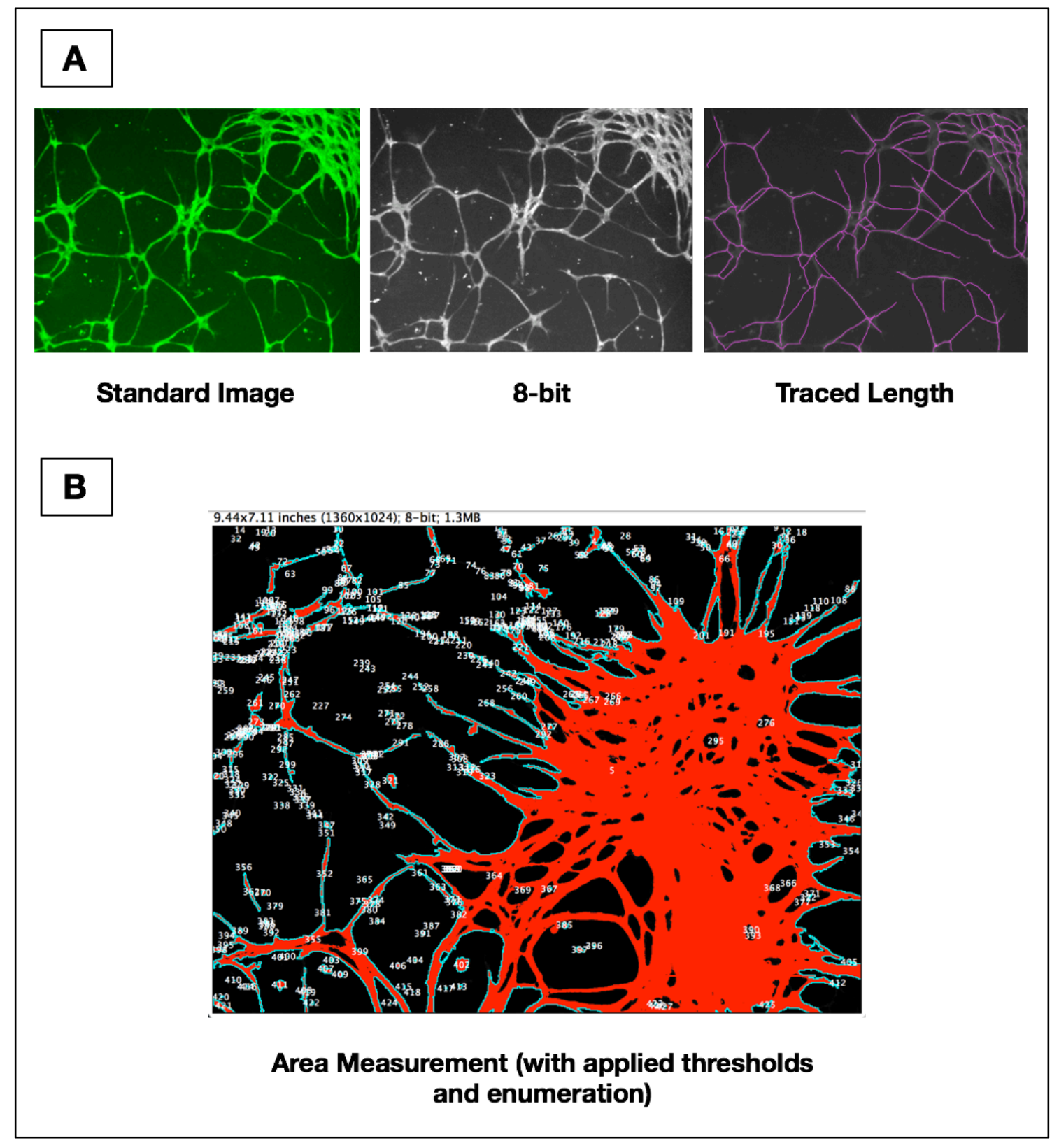

Figure 12. EC network tracing and area determination. (A) Image $J$ was used to convert standard UEAI ${ }^{+}$EC images to 8-bit. Images were then traced to measure total EC length using the NeuronJ plugin. (B) 8-bit images were also used for area measurement. Images were processed for size threshold (shown in red) and enumerated as described in the Materials and Methods. 


\section{RESULTS}

hSVF EC networks are reduced with increasing culture. hSVF is a heterogeneous single-cell suspension of ECs, fibroblasts, smooth muscle cells, macrophages, mural cells, and various stem cell populations ${ }^{9,297-299,369}$. Its inherent ability to rapidly and spontaneously self-assemble into a complex, hierarchical, and branched vasculature in vivo makes it a compelling candidate for therapeutic vascularization. We cultured hSVF as a means of interrogating potential mechanisms regulating vascular self-assembly ${ }^{9,381}$. After several days of culture, we observed the appearance of network-like structures in our cultures. Labeling of the cells with UEA1 (a lectin that binds human endothelium) and DAPI confirmed the presence of extensive EC networks in hSVF surrounded by an abundant stromal support system (Figure 13A).

Based on prior experience working with rodent and human SVF cells, we observed that high-passage SVF cells lose their ability to form vasculatures in vivo 9 . Therefore, we wanted to determine if this phenomenon also occurred in vitro. We performed an analysis of EC network formation as a function of passage, using hSVF cells from passages 1 through 4. Figure 13B shows that EC network density increased within a given passage over 5 days. Conversely, the overall EC density decreased with increasing passage. We observed significant reductions in normalized EC network length at day 5 as the passage number increased (Figure 13C). Normalized to P1, which exhibited the highest total EC length, P2, P3, and P4 cells exhibited lengths that were $0.59 \pm 0.02(p<0.001), 0.44 \pm 0.03(p<0.001)$, and $0.26 \pm 0.09(p=0.001)$ times as large, respectively. Thus, successive culturing decreases hSVF EC networking capacity. 


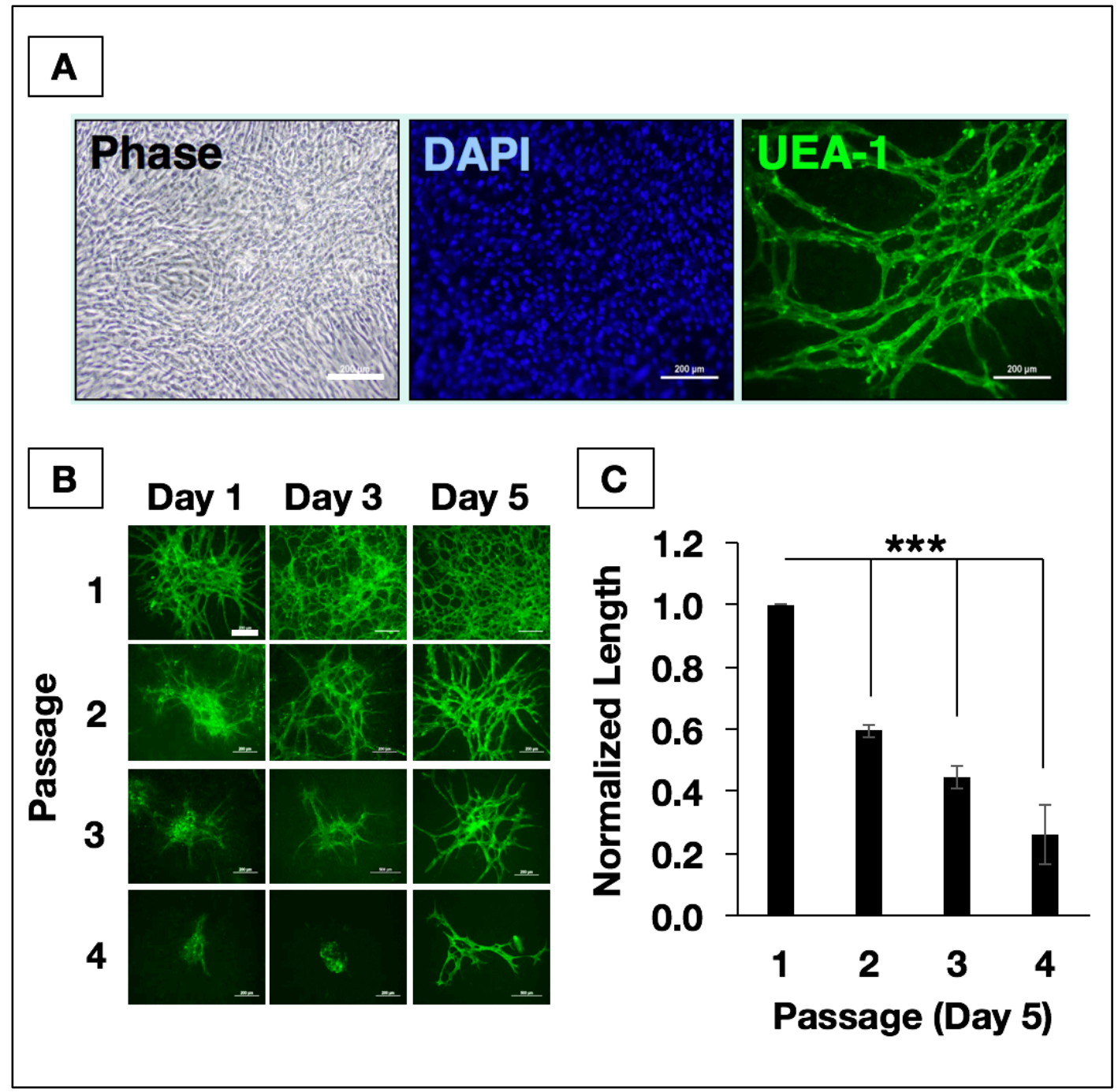

Figure 13. Culture reduces hSVF vascularization potential. (A) Within 5 days of culture, hSVF displayed complex EC networks, seen here after labeling cells with UEA1, a lectin that binds human endothelium. Scale $=10 x, 200 \mu m$. (B) hSVF networks increase in complexity over time in low passage culture, but rapidly loose EC networking capacity with subsequent passaging. Scale $=10 x, 200 \mu m$. (C) The average total hSVF EC length at day 5 was quantified and normalized to measurements of P1, day 5. Significant decreases in total hSVF hSVF EC length are seen by day 5 as a function of increasing passage $\left({ }^{* *} p \leq 0.001\right)$. Results shown as mean \pm S.E.M. 
While lower passages of hSVF achieved a greater quantified total EC network length by day 5 (Figure 13C), this self-assembly appears to be dependent on the number of UEA $1^{+}$EC within the culture at time of plating. As our analysis depends on having quantifiable EC networks, all subsequent in vitro and in vivo experiments utilized P1 cells due to their observed higher EC networking potential.

Wnt signaling is implicated hSVF EC network self-assembly. To identify the mechanisms regulating the self-assembly of adipose-derived hSVF into EC networks, we treated hSVF cultures with angiogenic inhibitors, including those for VEGF-R2 (ZM323881), PDGF-R $\beta$ (AG1296), HGF receptor/cMet (SU11274), the TGF- $\beta$ type-I receptor/Alk5 (SB431542) (Figure 11 and Table 1), and Wnt palmitoylation (IWP2) (Figure 14). In our preliminary tests, IWP2-induced inhibition yielded the most significant decreases in total EC length at the two highest concentrations (25 and $50 \mu \mathrm{M}$; data not shown). Because the role of Wnt signaling in vessel assembly is not as well characterized as it is in cancer, stem cell, and developmental biology, we focused our efforts here.

Before proceeding, we wanted to determine if our preliminary findings were due to IWP2-induced cytotoxicity (Figure 14A). At 50 $\mu \mathrm{M}$ IWP2, cell death was determined to be due to cytotoxic effects $(1.05 \pm 0.08 \mathrm{AU}$ with $p \leq 0.001)$, while the $25 \mu \mathrm{M}$ and lower concentrations displayed absorbance readings that were not significantly different from control cultures. Thus, we used the $25 \mu \mathrm{M}$ IWP2 dose for all subsequent experiments.

We next wanted to determine the effect of IWP2 concentration on hSVF network formation (Figure 14B.i). Treatment of hSVF with 6.25 or $12.5 \mu \mathrm{M}$ IWP2 showed no 


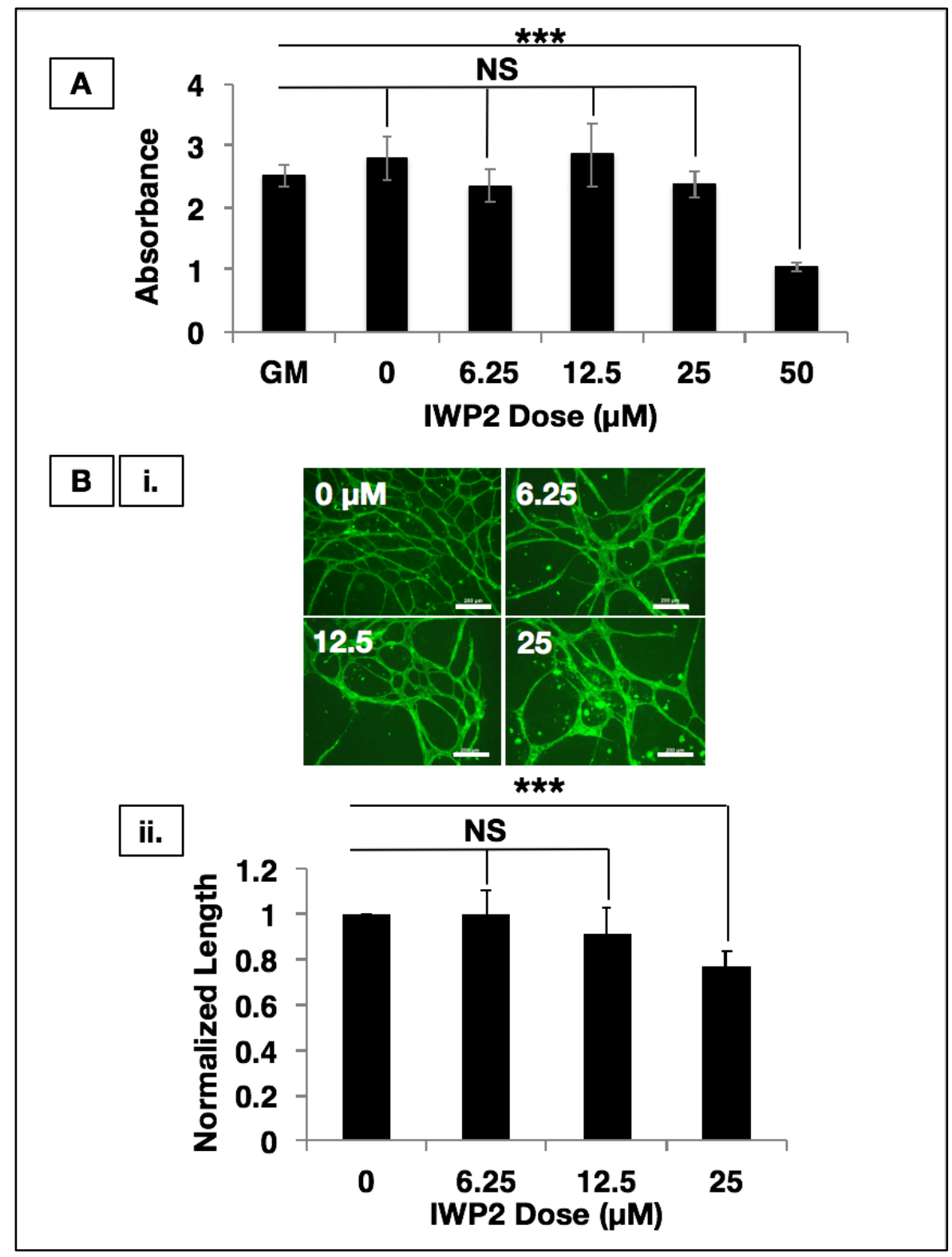

Figure 14. Wht mediates hSVF vascular self-assembly. (A) The CCK-8 cell cytotoxicity assay demonstrated that IWP2 concentrations $\leq 25 \mu M$ were not cytotoxic, whereas $50 \mu M$ was cytotoxic $\left({ }^{* *} p \leq 0.001\right)$. All subsequent experiments utilized $25 \mu M$. (B) (i.) Increasing IWP2 concentrations reduce hSVF EC networking, as visualized by UEA1 EC labeling. Scale $=10 x, 200 \mu m$. (ii.) Quantification of hSVF EC networks shows $25 \mu M$ IWP2 significantly decreases the total network length with respect to control $(* * * p \leq$ 0.001). $N S=$ not significant. Results shown as mean \pm S.E.M. 
changes in EC network formation compared to control cultures. However, after treating hSVF cells with $25 \mu \mathrm{M}$ IWP2, total EC network length was significantly reduced, measuring only $0.75 \pm 0.12$ times that of the untreated control $(p<0.001$; Figure 14B.ii). These findings demonstrate that inhibition of endogenous hSVF Wnt signaling has a downstream effect of reducing EC self-assembly and total EC network length.

Wnt5a and Fzd4 are expressed in hSVF. Since IWP2 blocks Wnt palmitoylation $^{376}$ and all Wnt isoforms are palmitoylated ${ }^{382}$, we wanted to determine which Wnt isoforms were expressed in hSVF. We isolated mRNA from hSVF cultures at days 1, 3, 5, and ran PCR for Wnt isoforms associated with angiogenesis ${ }^{383-392}$ (Figure 15A). In each experimental replicate, Wnt5a mRNA was consistently present with stable levels of expression over the 5 days of culture (Figure 16A). To determine if Wnt5a protein was present in hSVF, we immunolabeled cultures with anti-Wnt5a antibody and UEA1 (Figure 16B). We found that Wnt5a was abundant in the hSVF culture (Figure 16B.i), and that Wnt5a was not detectable in the fluorescent secondary antibody-only control (Figure 16B.ii).

Given that Wnt5a was present in the culture, we next wanted to determine which Wnt5a receptors were expressed in hSVF (Figure 15B). Wnt5a has been shown to interact with a number of receptors, including Frizzled-4 ${ }^{393},-5^{378,388,394}$, and ROR2 ${ }^{395,396}$. Similar to our Wnt PCR, we tested hSVF cDNA from day 1,3 , and 5 cultures for expression of these receptors. In certain replicates, we noticed a weak expression of FZD5 and ROR2 (Figure 15B), but in all replicates, we observed the consistent expression of FZD4 (Figure 16C), whose expression (like that of Wnt5a) was stable 


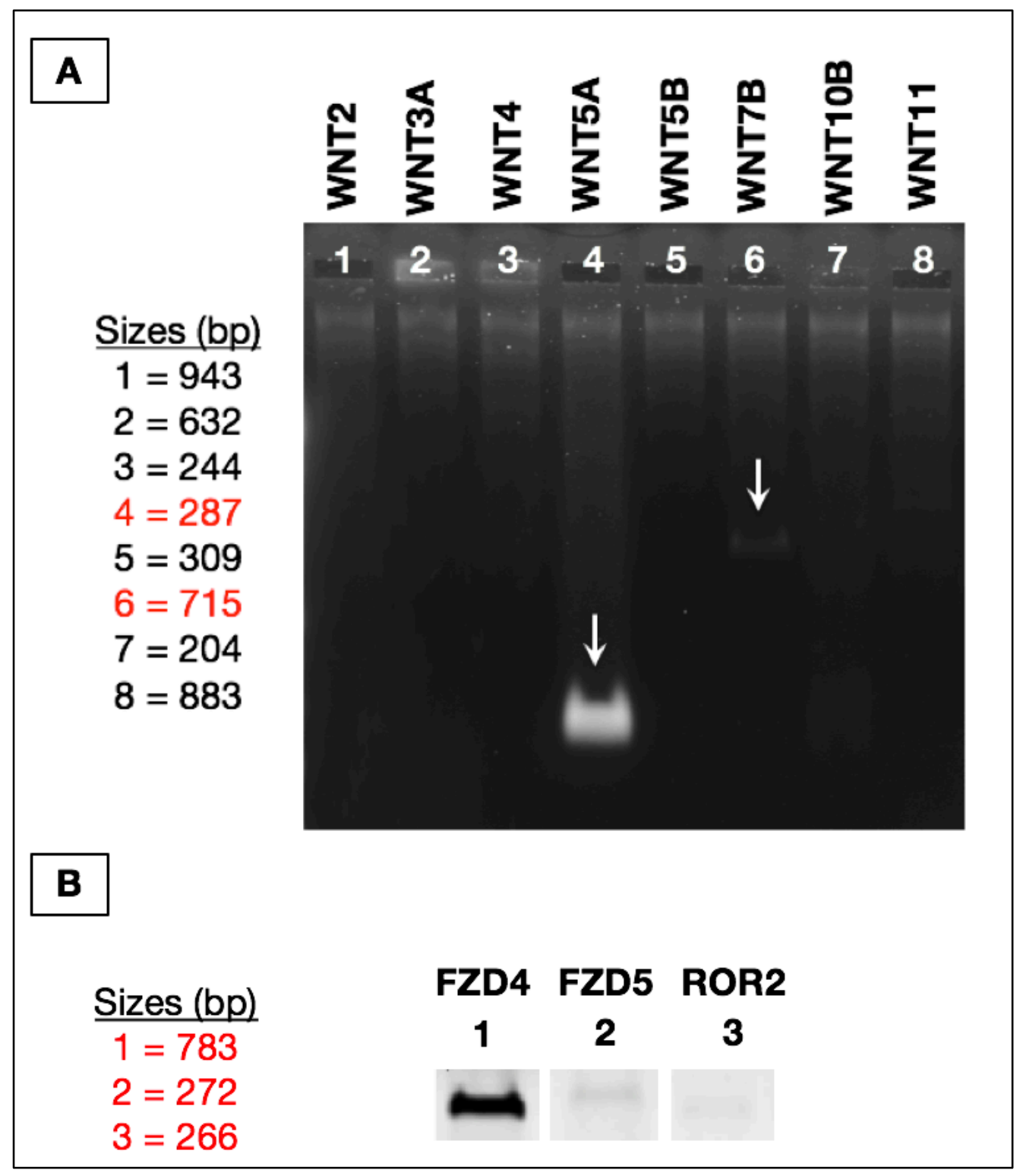

Figure 15. Wnt and Wnt5a-Receptor PCR Screening. (A) hSVF cDNA was initially screened by PCR using primers for Wnt isoforms associated with angiogenesis. Of these, WNT5A was most strongly expressed, while WNT7B was expressed to a lesser degree (arrows). (B) Once WNT5A was identified, WNT5A receptors underwent similar screening, with a focus on FZD4, FZD5, and ROR2. FZD4 was consistently expressed for all experiments, while FZD5 and ROR2 expression was inconsistent. 


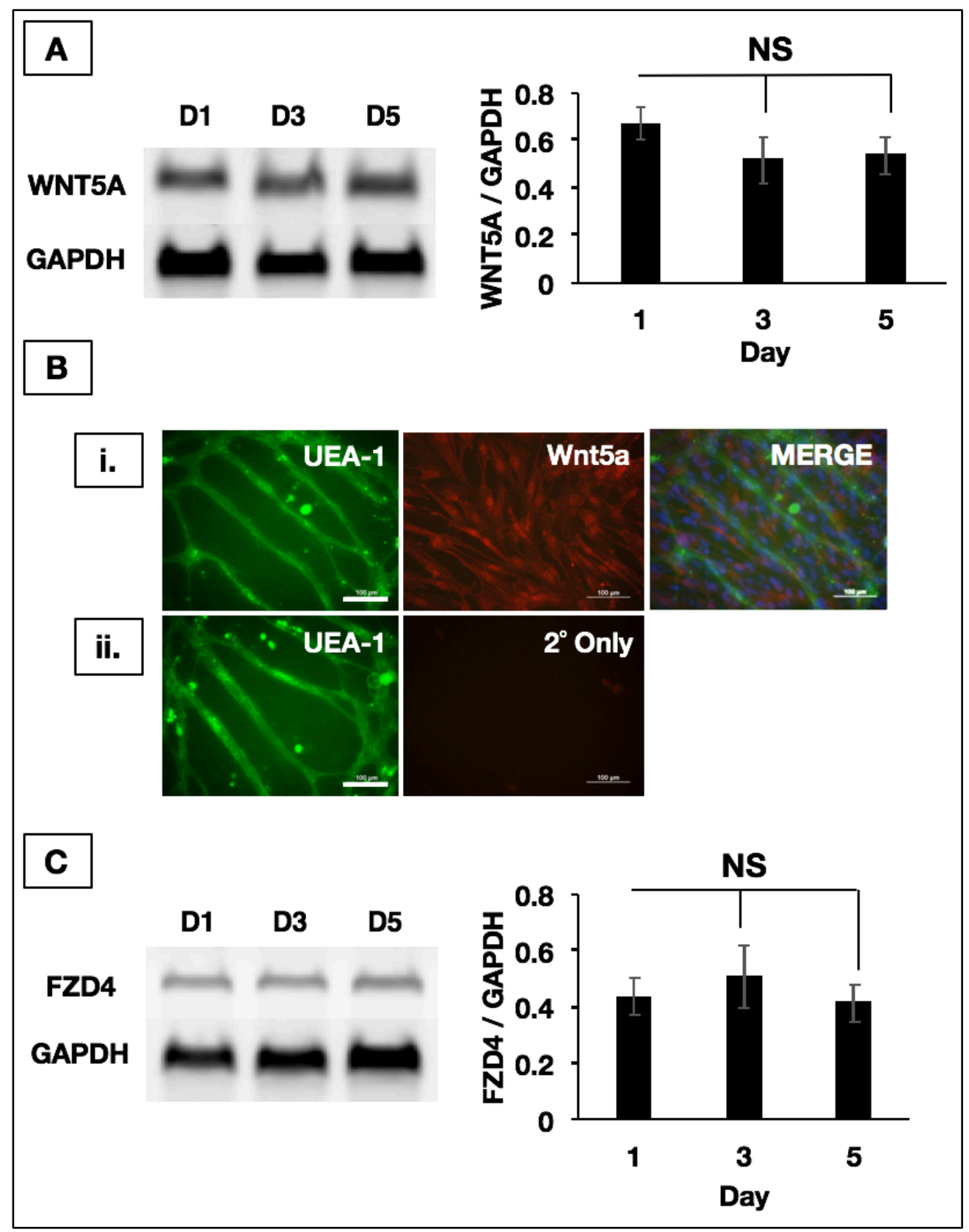

Figure 16. Wht5a and Fzd4 are highly expressed in hSVF cultures. (A) A time course of hSVF cDNA isolated was interrogated by RT-PCR for expression of WNT5A at days 1 , 3 and 5. WNT5A was robustly expressed at all time points. Normalization of WNT5A expression to the GAPDH loading control indicates stable expression of transcripts over 5 days. (B) (i.) hSVF was examined by immunocytochemistry for Wht5a expression. EC were labeled with UEAI (green), nuclei with DAPI (blue), and the entire culture with 
anti-Wnt5a $1^{\circ}+A F 5942^{\circ}$ antibodies (red). The merged image shows robust Wnt5a expression throughout the culture. Scale $=20 x, 100 \mu m$. (ii.) A negative control run in parallel used only the AF594 $2^{\circ}$ antibody to rule out non-specific fluorophore binding. Scale $=20 x, 100 \mu \mathrm{m}$. (C) Wnt5a receptor expression was also tested by RT-PCR (also see Figure 25B). FZD4 was stably and consistently expressed, as shown by gel electrophoresis. Densitometry demonstrates no change in FZD4 transcripts over 5 days. $N S=$ not significant. Results shown as mean \pm S.E.M. 
through day 5. These findings confirm the expression of Wnt5a and its receptor Frizzled4 within cultured hSVF and suggest a possible mechanism for regulating hSVF EC selfassembly.

Recombinant Wnt5a mediates the self-assembly of hSVF EC networks in IWP2-treated cultures. We next wanted to determine if Wnt5a played a specific role in the self-assembly of hSVF EC networks. hSVF cultures were exposed to a combination of $25 \mu \mathrm{M}$ IWP2 and varying concentrations $(0-150 \mathrm{ng} / \mathrm{ml})$ of exogenously supplied recombinant human Wnt5a protein (rhWnt5a; Figure 17). Since IWP2 (a) inhibits the release of all endogenous Wnt isoforms from hSVF cells without affecting cognate receptors and (b) impairs hSVF EC networking, we wanted to determine if the selective addition of rhWnt5a alone would restore hSVF EC network assembly.

After five days of rhWnt5a co-administration with IWP2, we labeled hSVF cultures with UEA1 (Figure 17A) and quantified the magnitude of hSVF EC networking. As expected, the addition of $25 \mu \mathrm{M}$ IWP2 alone (negative control, with no supplemental rhWnt5a) inhibited EC network assembly. Supplementing the cultures with as little as $0.75 \mathrm{ng} / \mathrm{ml} \mathrm{rhWnt5a}$ yielded significant increases in $\mathrm{UEAl}^{+}$area (Figure 17B) compared to cultures given IWP2 alone, resulting in a $2.44 \pm 0.36$-fold increase $(p \leq 0.01)$. The 7.5 $\mathrm{ng} / \mathrm{ml}$ concentration elicited the largest increase in area, measuring $3.34 \pm 0.87$ times $(p \leq$ 0.05 ) larger than cultures treated with IWP2 alone, while $75 \mathrm{ng} / \mathrm{ml}$ elicited a $2.93 \pm 0.76$ fold increase $(p \leq 0.05)$ and $150 \mathrm{ng} / \mathrm{ml}$ yielded an increase of $2.98 \pm 0.72$ fold $(p \leq 0.05)$. ANOVA analysis further indicated that treatment with any concentration of rhWnt5a was significant compared to controls lacking rhWnt5a, but that there were no significant differences between the various rhWnt5a treatments. 


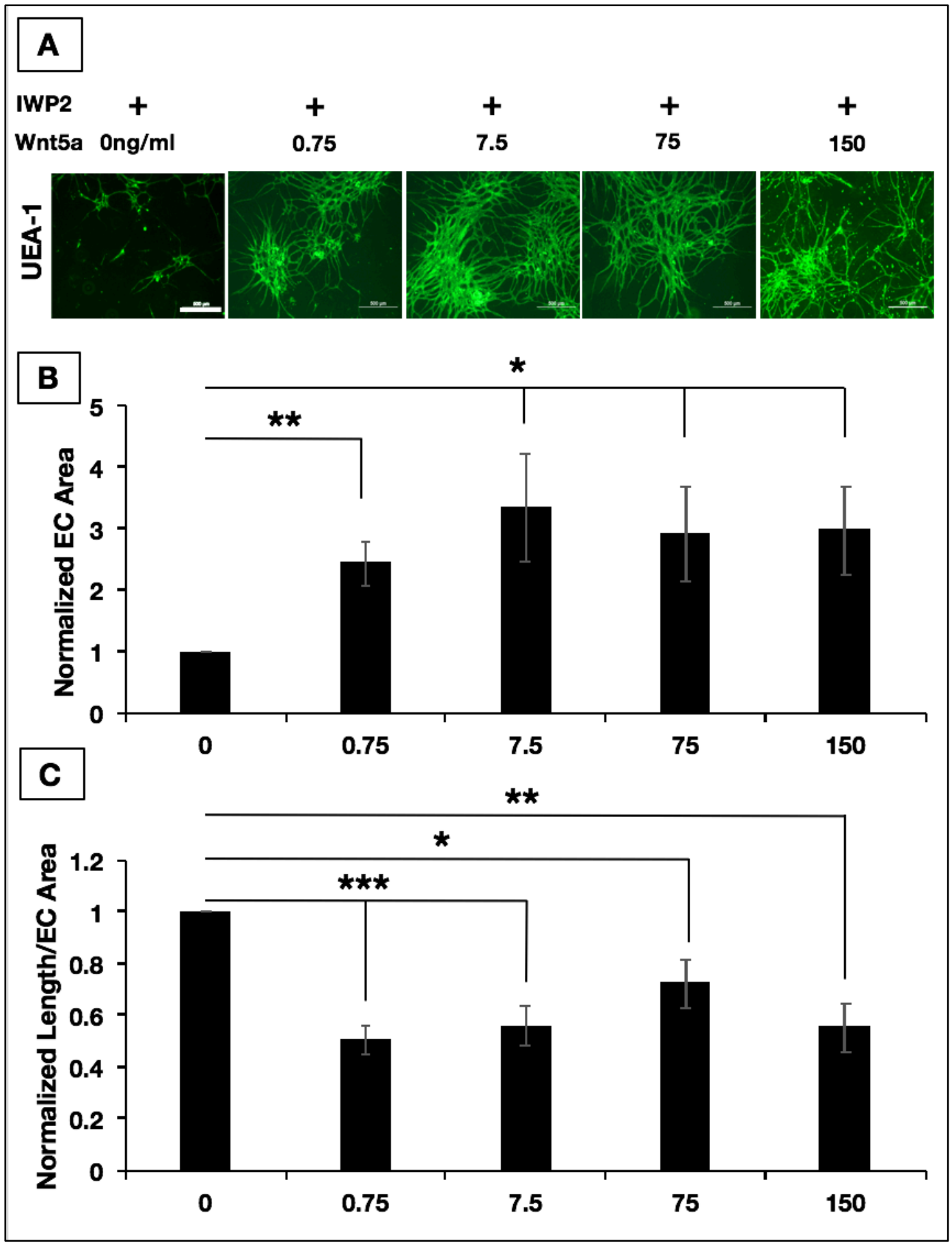

Figure 17. Exogenous recombinant Wnt5a mediates hSVF vascular self-assembly during IWP2 inhibition. All hSVF cultures were treated with $25 \mu M$ IWP2 and given increasing concentrations of rhWnt5a. (A) Representative images of $\mathrm{UEAl}^{+}$(green) labeling of hSVF EC networks are seen for all conditions. Scale Bar $=4 x, 500 \mu m$. (B) 
The $U E \mathrm{Al}^{+}$area for each rhWnt5a-treated condition was normalized to the non-treated 0 $n g / m l$ rhWnt5a control. rhWnt5a significantly increased the total $\mathrm{UEA1}^{+}$area compared to the control $\left({ }^{*} p \leq 0.05 ; *^{*} p \leq 0.01\right)$. (C) The total UEA1 ${ }^{+}$network length was normalized to the total $\mathrm{UEAl}^{+}$area. Compared to the $0 \mathrm{ng} / \mathrm{ml}$ rhWnt5a control, supplemental rhWnt5a produced areas of hSVF EC sheeting, which is characterized by lower ratios of total EC length to EC area. Results shown as mean \pm S.E.M. 
Interestingly, while addition of rhWnt5a significantly increased $\mathrm{UEA} 1^{+} \mathrm{EC}$ area in the presence of IWP2, it also promoted the aggregation of EC in monolayers that resemble sheets To quantitatively characterize this, we measured total EC network length in each field-of-view and normalized this length to the measured EC area in that same field-of-view (Figure 17C). Compared to cultures given IWP2 alone, the addition of 0.75 $\mathrm{ng} / \mathrm{ml} \mathrm{rhWnt5a}$ yielded ratios that were only $0.51 \pm 0.06$ times as large $(p \leq 0.001)$. Similarly, the $7.5,75$, and $150 \mathrm{ng} / \mathrm{ml}$ concentrations exhibited ratios that were $0.56 \pm 0.08$ ( $p \leq 0.001), 0.72 \pm 0.1(p \leq 0.05)$, and $0.55 \pm 0.09(p \leq 0.001)$ times that seen with the control. Thus, considering that rhWnt5a increased EC area and reduces the ratio of total EC length to EC area, rhWnt5a appears to specifically play a role in modulating hSVF vascular network assembly in a process that depends on EC proliferation.

Wnt signaling is divided into canonical and non-canonical pathways, which utilizes $\beta$-catenin or non- $\beta$-catenin pathways to transduce the signal ${ }^{397}$. Wnt5a utilizes multiple receptors but is generally categorized as a non-canonical $\mathrm{Wnt}^{379,398}$. To determine if the same effect on hSVF EC networking could be produced by a different Wnt, we repeated the above experiment using the prototypical canonical Wnt, Wnt3a at the same concentrations as those used for the rhWnt5a in vitro experiment (Figure 18A). The quantification of the total hSVF EC area normalized to the $0 \mathrm{ng} / \mathrm{ml}$ Wnt3a control indicated that Wnt3a supplementation did not significantly increase networking area (Figure 18B) or the ratio of EC network length to EC area of IWP2-treated cultures (Figure 18C). Further, ANOVA analysis demonstrated that treatment with any concentration of Wnt3a did not yield a significant outcome. These findings suggest that Wnt5a alone can partially restore the assembly of hSVF EC networking when 


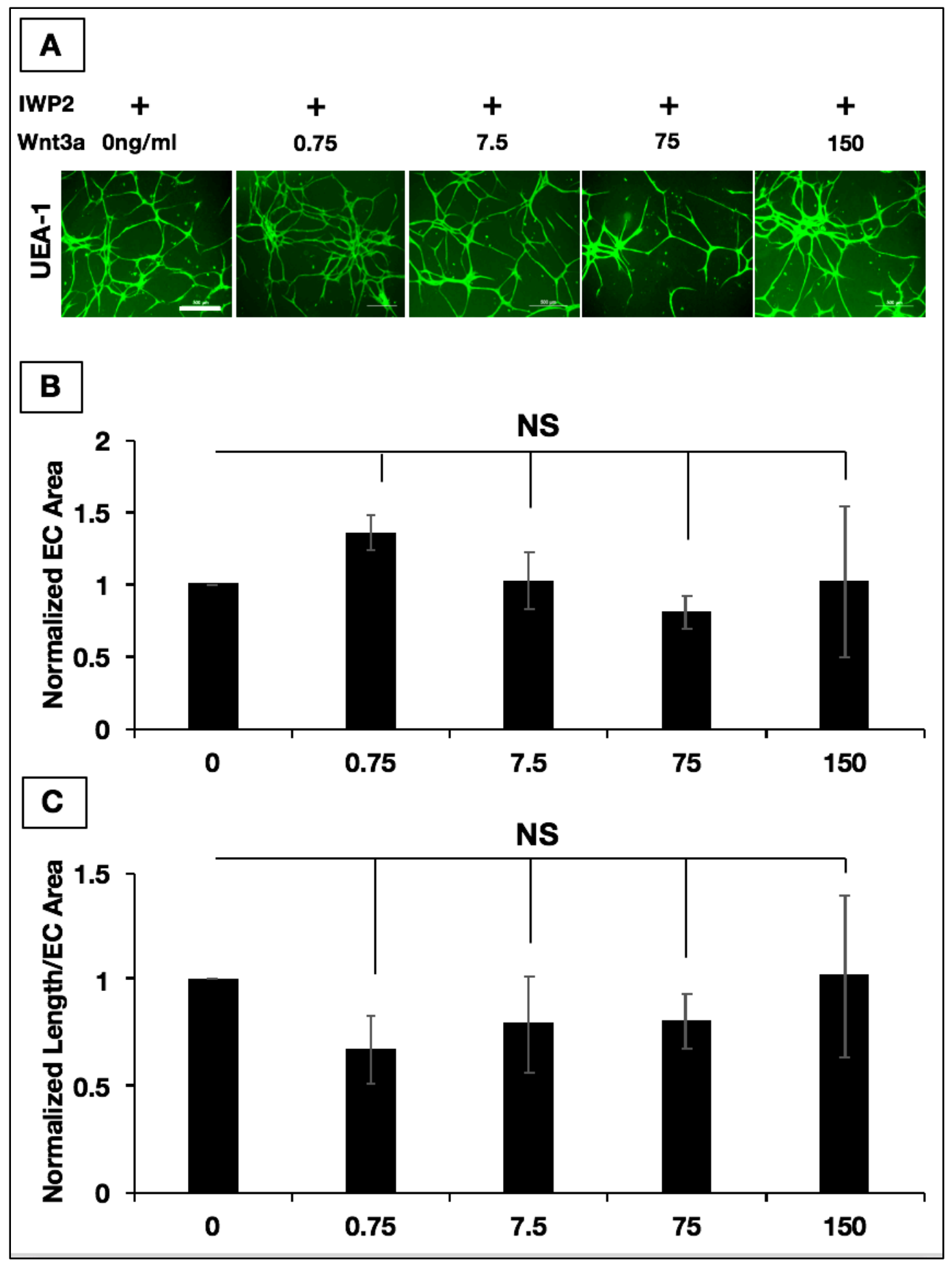

Figure 18. IWP2-treated hSVF EC networks are not affected by supplemental rhWnt3a. (A) hSVF was treated with $25 \mu M I W P 2$ for $5 d$ and supplemented with varying concentrations of rhWnt3a, except where noted. Human EC were labeled with UEA1 (green), imaged and quantified. (B) The total UEA $1^{+} E C$ area seen with each treatment 
was normalized to that for the untreated IWP2 control. Quantification demonstrates that rhWnt3a supplementation yielded no significant changes to network area compared to the IWP2 control. (C) Likewise, the normalization of total UEA1 ${ }^{+}$EC network length to the total UEAI ${ }^{+}$EC area was not significantly affected by exogenous rhWnt3a addition. $N S=$ not significant. Results shown as mean \pm S.E.M. 
endogenous Wnt release is prevented. It also demonstrates that the effect on network assembly is not universally mediated by all Wnt isoforms, since Wnt3a substitution was ineffective.

\section{Wnt5a mediates hSVF microvascular self-assembly in vivo. Our data supports} that Wnt5a plays a specific role in hSVF microvascular self-assembly in vitro. Therefore, we wanted to determine its role in the self-assembly that others and we have reported in vivo ${ }^{1,9,299}$. We initially exposed hSVF cultures to varying concentrations $(0-20 \mu \mathrm{g} / \mathrm{ml})$ of Wnt5a neutralizing antibody (referred to as anti-Wnt5a) ${ }^{377,378,399}$ to determine an appropriate concentration for use (Figure 19). Compared to the negative control (0 $\mu \mathrm{g} / \mathrm{ml}$ ), there were no significant differences observed with 5 or $10 \mu \mathrm{g} / \mathrm{ml}$ anti-Wnt5a, while treatment with $20 \mu \mathrm{g} / \mathrm{ml}$ significantly reduced the total EC length $(0.68 \pm 0.06$ fold, $p \leq 0.05$; Figure 19A) and segment number $(0.52 \pm 0.09$ fold, $p \leq 0.05$; Figure 19B $)$. The addition of an IgG control antibody at the same concentrations did not affect total EC length or segment number (data not shown).

Based on these in vitro findings, we utilized $20 \mu \mathrm{g} / \mathrm{ml}$ anti-Wnt5a in subsequent in vivo experiments to determine if Wnt5a modulated hSVF microvascular assembly in that setting as well. We combined hSVF in 3D collagen-I constructs with $20 \mu \mathrm{g} / \mathrm{ml} \operatorname{IgG}$ isotype control antibody, $20 \mu \mathrm{g} / \mathrm{ml}$ anti-Wnt5a neutralizing antibody, or $7.5 \mathrm{ng} / \mathrm{ml}$ rhWnt5a (Figure 20). After implantation for two weeks, host animals were sacrificed and the constructs explanted, processed and analyzed as described in the Materials and Methods (Figure 20A). Each experimental condition was quantified for length density (i.e., a ratio of the total EC length to the 3D scan volume; Figure 20B) as well as the 


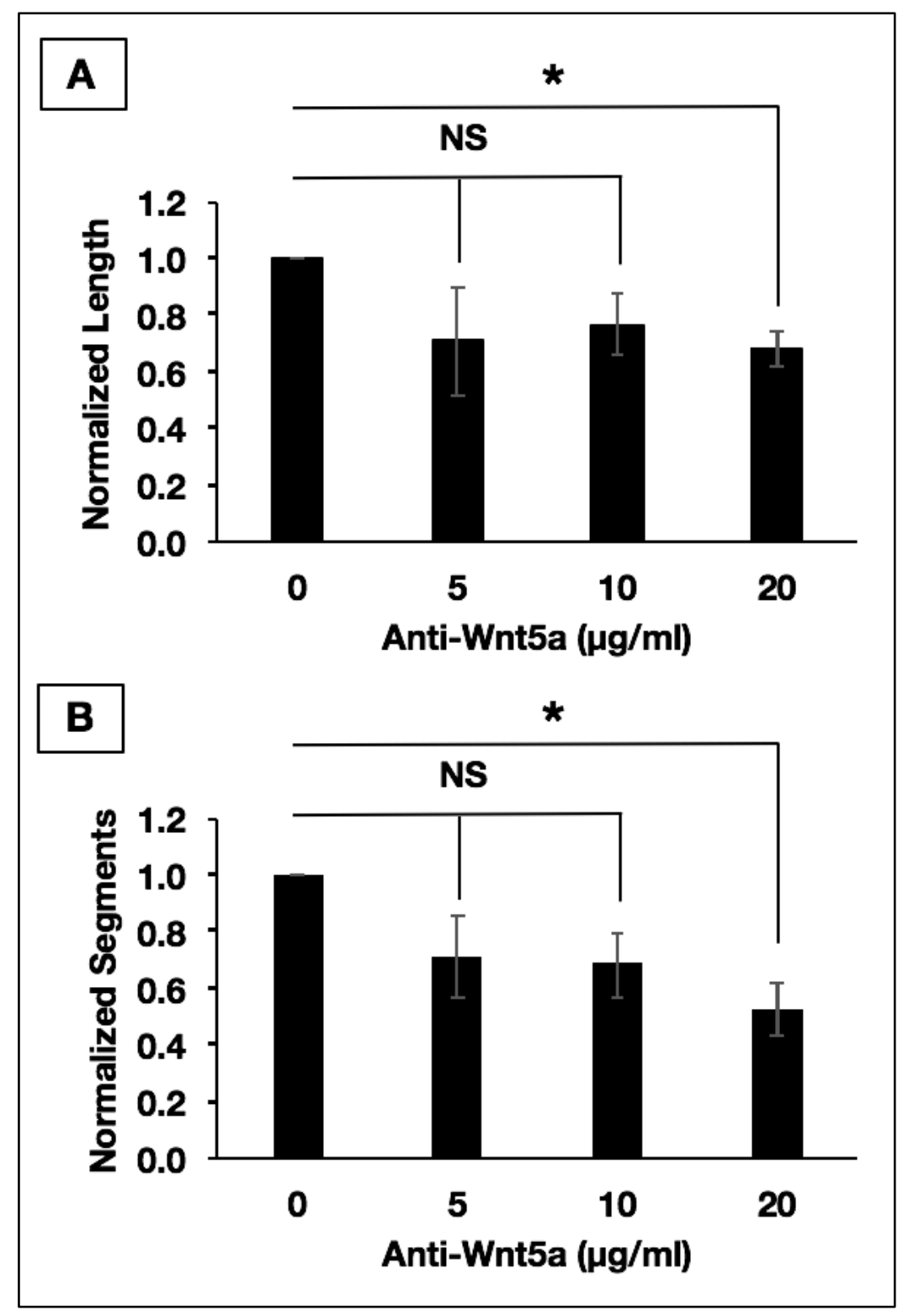

Figure 19. Wnt5a neutralizing antibody inhibits hSVF total EC length and segments. The largest concentration of anti-Wnt5a $(20 \mu \mathrm{g} / \mathrm{ml})$ significantly reduced the (A) $h S V F$ EC total length $\left({ }^{*} p \leq 0.05\right)$ and $(\boldsymbol{B})$ number of segments $\left({ }^{*} p \leq 0.05\right)$. This concentration was used to examine Wnt5a's role in hSVF vascular self-assembly in vivo. In both (A) and (B), values obtained for length and segments with each treatment were normalized to the $0 \mu \mathrm{g} / \mathrm{ml}$ control. $N S=$ not significant. Results shown as mean \pm S.E.M. 


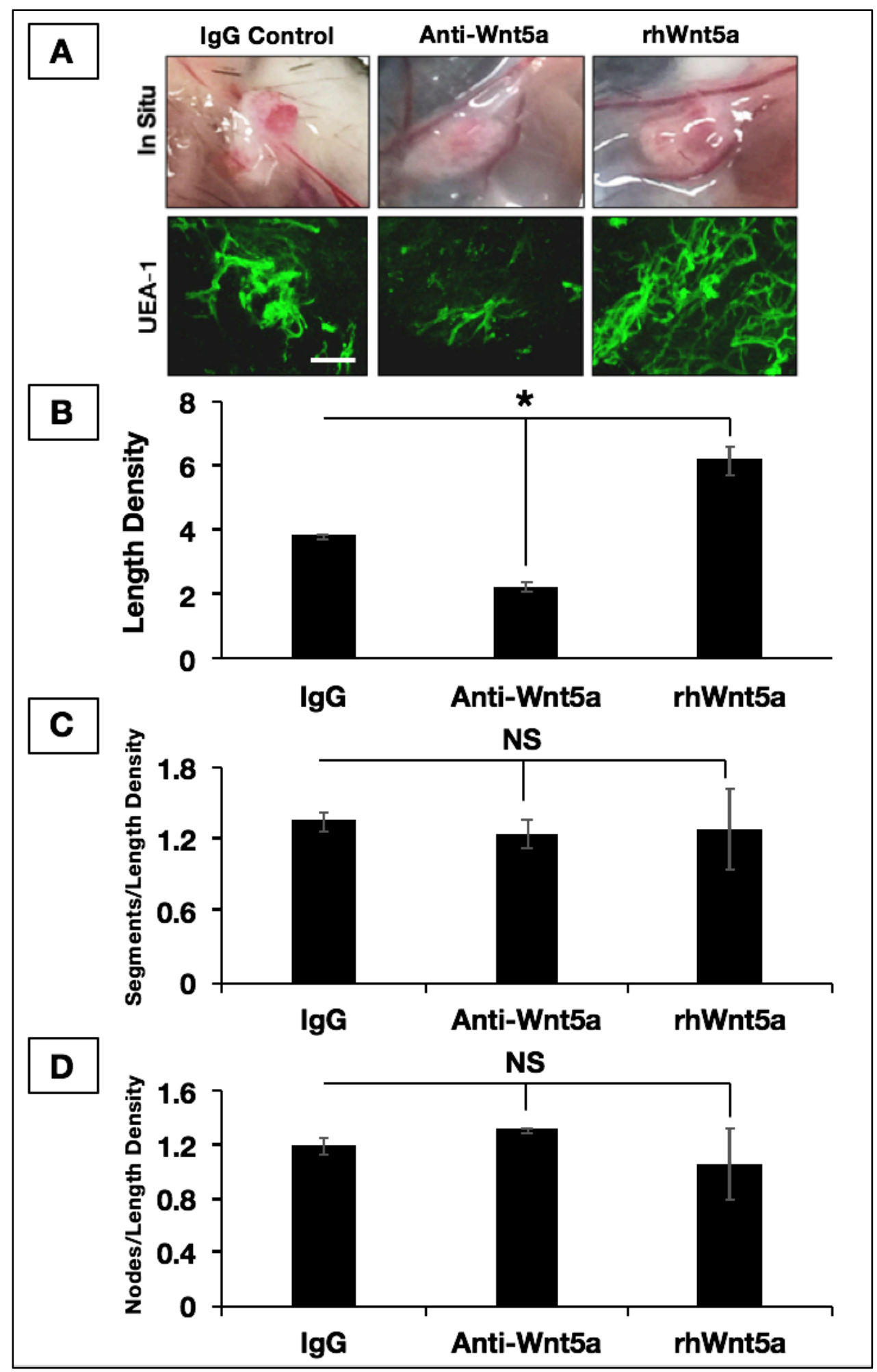

Figure 20. Wht5a drives hSVF EC microvascular assembly in vivo. (A) $3 D$ collagen-I constructs containing hSVF with $20 \mu \mathrm{g} / \mathrm{ml} \mathrm{IgG}$ isotype control, $20 \mu \mathrm{g} / \mathrm{ml}$ Wnt5a 
neutralizing antibody (anti-Wnt5a), or $7.5 \mathrm{ng} / \mathrm{ml}$ rhWnt5a were subcutaneously implanted in immunodeficient mice for $2 w$. In situ images show $2 w$ constructs before harvest. Constructs were labeled with UEA1 (green) and imaged by confocal microscopy. Scale bar $=20 x, 100 \mu m$. (B) The total EC length was normalized to the $3 D$ scan volume (length density). Construct treatment with anti-Wnt5a exhibited a significantly reduced length density $\left((2.25 \pm 0.16) \times 10^{-5} \mu \mathrm{m} / \mu \mathrm{m}^{3} ; *^{*} \leq 0.05\right)$ compared to the IgG control $\left((3.81 \pm 0.1) \times 10^{-5} \mu \mathrm{m} / \mu \mathrm{m}^{3}\right)$. Conversely, rhWnt5a significantly increased this ratio $\left((6.17 \pm 0.43) \times 10^{-5} \mu \mathrm{m} / \mu \mathrm{m}^{3} ; * p \leq 0.05\right)$ compared to the control. (C) The normalization of segment number to length density showed no significant affect with either the antiWnt5 a treatment $\left((1.24 \pm 0.13) \times 10^{7}\right.$ segments $\left./ \mu m / \mu m^{3}\right)$ or the rhWnt5a treatment $((1.28$ $\pm 0.34) \times 10^{7}$ segments $\left./ \mu m / \mu m^{3}\right)$ compared to $\operatorname{IgG}$ controls $\left((1.34 \pm 0.07) \times 10^{7}\right.$ segments $/ \mu \mathrm{m} / \mu \mathrm{m}^{3}$ ). (D) The ratio of node number to length density also shows no significant difference between the IgG control $\left((1.19 \pm 0.07) \times 10^{7}\right.$ nodes $\left./ \mu \mathrm{m} / \mu \mathrm{m}^{3}\right)$, the anti-Wnt5a treatment $\left(1.31 \pm 0.02\right.$ nodes $\left./ \mu \mathrm{m} / \mu \mathrm{m}^{3}\right)$, and treatment with $\mathrm{rhWnt5a}((1.06 \pm$ 0.27) $\times 10^{7}$ nodes $\left./ \mu m / \mu m^{3}\right) . N S=$ not significant. Results shown as mean \pm S.E.M. 
ratios of segment (Figure 20C) and node (Figure 20D) numbers to length density. Thus, length density is a measure of assembly, while the segment and node ratios serve to characterize hSVF vascular complexity.

IgG controls exhibited an average length density of $(3.81 \pm 0.1) \times 10^{-5} \mu \mathrm{m} / \mu \mathrm{m}^{3}$. Treatment with anti-Wnt5a significantly reduced the length density $\left((2.25 \pm 0.16) \times 10^{-5}\right.$ $\left.\mu \mathrm{m} / \mu \mathrm{m}^{3} ; p \leq 0.05\right)$, while rhWnt5a significantly increased this ratio $\left((6.17 \pm 0.43) \times 10^{-5}\right.$ $\left.\mu \mathrm{m} / \mu \mathrm{m}^{3} ; p \leq 0.05\right)$. Put a different way, compared to the IgG control, anti-Wnt5a reduced total vascular length by approximately $41 \pm 5.6 \%$, while rhWnt5a increased overall vascular length by approximately $62 \pm 15 \%$.

With regards to vascular complexity, the addition of anti-Wnt5a to $3 \mathrm{D}$ implants did not significantly affect the ratio of segments to length density $\left((1.24 \pm 0.13) \times 10^{7}\right.$ segments $\left./ \mu \mathrm{m} / \mu \mathrm{m}^{3} ; p=0.56\right)$ compared to $\operatorname{IgG}$ controls $\left((1.34 \pm 0.07) \times 10^{7}\right.$ segments $/ \mu \mathrm{m} / \mu \mathrm{m}^{3}$ ). Anti-Wnt5a also bore no significant effect on the ratio of nodes to length density, with $(1.31 \pm 0.02) \times 10^{7}$ nodes $/ \mu \mathrm{m} / \mu \mathrm{m}^{3}(p=0.22)$ compared to the $(1.24 \pm$ 0.07) $\times 10^{7}$ nodes $/ \mu \mathrm{m} / \mu \mathrm{m}^{3}$ seen with the IgG control. Also, rhWnt5a did not significantly influence complexity either $\left((1.28 \pm 0.34) \times 10^{7}\right.$ segments $/ \mu \mathrm{m} / \mu \mathrm{m}^{3}(p=0.86)$ and $(1.06 \pm$ $0.27) \times 10^{7}$ nodes $\left./ \mu \mathrm{m} / \mu \mathrm{m}^{3}(p=0.67)\right)$. These results also correlate with the qualitative observation that 3D implants did not exhibit noticeable EC sheeting.

ANOVA analysis indicated that the anti-Wnt5a and rhWnt5a treatments only imparted significant effects on EC lengths within a 3D construct. Thus, this data demonstrates that in a 3D implant, the supplementation of rhWnt5a in vivo significantly increased the overall length of the hSVF microvasculature, while the specific blockade of Wnt5a with a neutralizing antibody significantly reduced hSVF cells' capability to form a 
microcirculation. Together, these findings support a role for Wnt5a in mediating hSVF microvascular self-assembly in vivo.

\section{DISCUSSION}

The main and novel findings of this report are that (a) human adipose SVF (hSVF) spontaneously forms endothelial networks in vitro, but loses this capacity due to a reduction in system endothelium, (b) pan-inhibition of hSVF-endogenous Wnt isoforms by IWP2 significantly reduces EC networking, (c) the addition of exogenous Wnt5a, but not Wnt3a, to IWP2-treated cultures modulates EC network assembly in vitro, (d) the spontaneous assembly of hSVF microvascular networks in vivo is inhibited by a Wnt5a neutralizing antibody and enhanced by addition of recombinant human Wnt5a protein, and (e) Wnt5a does not influence the complexity of hSVF-derived microvessels.

Adipose SVF is a heterogeneous cell population that contains EC, perivascular cells, macrophage, fibroblasts and stem cells ${ }^{9,369,400}$. This endogenous heterogeneity is likely what imbues the cellular system with the specific capacity to form functional microvasculatures in vivo ${ }^{1,9,370}$. In prior efforts, we leveraged this functionality to support the engraftment of hepatic parenchymal cells ${ }^{1,9}$. Koh and colleagues demonstrated that the formation of vascular networks by mouse SVF (mSVF) depended on the presence of EC and is VEGF-dependent ${ }^{299}$. We reported that the removal of EC from rat SVF cells yielded a significantly altered vascular interaction ${ }^{9}$. These findings support the hypothesis that the heterogeneity of SVF is crucial to its biological functionality. However, the mechanisms regulating this functionality are poorly defined. 
It is a common practice to subculture adipose SVF to isolate the putative ADSC 401. This "pure" population of mesenchymal cells is explicitly void of endothelium (i.e. $\left.\mathrm{CD} 31^{-}\right)^{402}$. In early culture, the endogenous EC population is enriched and capably forms robust EC networks. This capacity diminishes rapidly with each successive passage. The reasons for this decrease are not clear, but could be due to EC depletion by our sequential network assays, differential proliferation rates between EC and stromal cells favoring the latter, or culture-induced loss by EC phenotypic drift. There are multiple reports in the literature of ADSC combined with various EC populations to create a synthetic vascular and stromal cell system ${ }^{298,403,404}$. Our data supports the conclusion that the hSVF cellular system is a complex heterogeneous cell mixture that endogenously retains the functional capacity for microvascular self-assembly, which the artificially generated EC-ADSC coculture may not fully replicate.

To determine the mechanisms that regulate hSVF EC network self-assembly in vivo, we investigated several angiogenesis-associated pathways. Inhibition of receptors for VEGF and PDGF-B significantly reduced hSVF EC networking, while blockade of the TGF $\beta 1$ receptor Alk5 and HGF receptor c-Met did not. This data agrees with Koh, et al. ${ }^{299}$ with regards to the role of VEGF and further suggests that a blockade of PDGFB/PDGFR- $\beta$ signaling may prevent perivascular cell recruitment as demonstrated in other vascular beds ${ }^{405-408}$. The lack of effect from Alk5 inhibition may be due to (a) TGF $1 /$ Alk5 having a role in later stages of vascular stabilization ${ }^{348,409}$, or (b) TGF $\beta 1$ could be signaling through Alk1 on $\mathrm{EC}^{410,411}$. In other model systems, we demonstrated that HGF/c-Met signaling modulates vascular assembly and angiogenesis ${ }^{9,316}$. The lack of effect on hSVF could be due to limitations of our hSVF in vitro assay to mimic the stages 
when $\mathrm{HGF} / \mathrm{c}-\mathrm{Met}$ are active. Our battery of inhibitors also included the Wnt palmitoylation inhibitor IWP $2^{376}$, which showed a significant effect on hSVF network self-assembly. Though Wnt biology is extensively studied in other contexts, its functions in EC biology and angiogenesis have only recently been investigated ${ }^{392,399,412-414}$ with defined roles for canonical and non-canonical Wnt isoforms ${ }^{388,392,412}$ in vascularization.

Since IWP2 inhibits the release of all Wnt isoforms, we tested hSVF for expression of Wnt isoforms previously affiliated with angiogenesis. Since our PCR panel identified Wnt5a as being highly expressed within hSVF, we hypothesized that Wnt5a mediates hSVF EC network assembly. The addition of various concentrations of exogenous rhWnt5a significantly increased the areas of IWP2-inhibited EC networks, though these treatments also significantly reduced the ratios of lengths to EC areas. These effects were not seen when the canonical rhWnt3a was supplemented in place of rhWnt5a. Together, these findings suggest that while Wnt5a is an essential modulator of hSVF EC network assembly in vitro, other Wnt isoforms (alone or in conjunction with Wnt5a) likely modulate vascular complexity (characterized by reduced EC sheeting and increased branching).

To determine if our in vitro findings provided the mechanisms controlling in vivo microvascular self-assembly, we implanted $\mathrm{hSVF}^{1,9,370}$ in $3 \mathrm{D}$ constructs supplemented with a Wnt5a-specific neutralizing antibody ${ }^{378,379,415}$, rhWnt5a, or IgG isotype control. Compared to IgG controls, implants containing anti-Wnt5a exhibited reduced the volumetric total vessel length, whereas the numbers of nodes and segments per unit of length density were essentially unchanged. To contrast, constructs containing rhWnt5a exhibited a significantly increased length density, but like anti-Wnt5a, rhWnt5a had little 
effect on the numbers of segments and nodes per unit of length density. This suggests that Wnt5a does not influence the complexity of the resulting hSVF-derived vascular system. This data agrees with the results from other reports in the literature that Wnt5a signaling modulates EC vascular assembly ${ }^{392}$, but this is the first time that this has been demonstrated in hSVF - a clinically relevant, natively heterogeneous population.

Our in vivo findings also correspond with those seen in vitro, in spite of the fact that in vitro experiments utilized IWP2 (the pan-Wnt release inhibitor) and in vivo experiments did not. It is possible that a bolus delivery of rhWnt5a in a 3D implant could decrease, but not necessarily eliminate, the influence of other Wnt isoforms released by hSVF cells or host tissues, since certain Wnt isoforms can compensate for one another in development $^{416}$. While rhWnt5a did not significantly influence hSVF microvascular complexity in vivo (potentially based on a compensatory, decreased influence of other Wnt isoforms), the incidence of EC sheeting in vivo appeared to have been mitigated (perhaps due to the basal presence of other Wnt isoforms or other biomolecules associated with angiogenesis). Though this seems paradoxical, it is clear that Wnt5a is only one constituent of a complex system that imparts control over hSVF spontaneous microvascular self-assembly.

A key consideration in our experiments is that we obtained our hSVF cells from three different patient sources of discarded human lipoaspirate. Undoubtedly, the characteristics of adipose exhibits variability between patients. The cellular composition of each hSVF batch is likely to be different depending on factors such as gender, age, and social lifestyle (e.g. smoking, alcohol use), all of which likely have significant effects on cell viability and cellular function., Prior studies have indicated that the proportion of 
$\mathrm{CD} 1^{+} \mathrm{EC}$ is decreased with culture SVF cells as opposed to freshly isolated cells ${ }^{9,400}$. In spite of these factors, we observed the same trends with each hSVF isolation, regardless of the patient demographics. This supports our finding that Wnt5a signaling may play a general role in mediating hSVF microvascular assembly in vivo. At this stage, it is unclear how Wnt5a may be involved. We show robust expression of Wnt5a within hSVF, but it is unknown if there is a specific cell lineage within hSVF that is responsible for affecting the EC response. We show robust expression of FZD4 in hSVF and Wnt5a utilizes this receptor in $\mathrm{EC}^{393}$, but Wnt5a also signals through FZD5 ${ }^{388}$ and ROR2 ${ }^{396,417}$. Thus, the downstream signaling events still need to be defined. Though more work is required to understand how the hSVF cellular system functions to self-assemble a microvasculature, our data identifies Wnt5a signaling as a component that potentiates this process.

In this endeavor, our goal was to define the mechanisms controlling hSVF spontaneous EC microvascular network formation in vivo. Our findings are novel, as this is the first report that identifies Wnt5a as a modulator of hSVF vascular assembly. This work underscores the fact that adipose SVF is a potential regenerative cell system. Understanding how the mixture of heterogeneous cell types within hSVF works together could enhance efforts to better vascularize tissues or promote the engraftment of engineered therapeutic cells ${ }^{1,9}$. 


\author{
CHAPTER V \\ RESTORATION OF PHYSIOLOGICALLY RESPONSIVE LOW-DENSITY \\ LIPOPROTEIN RECEPTOR-MEDIATED ENDOCYTOSIS IN GENETICALLY \\ DEFICIENT INDUCED PLURIPOTENT STEM CELLS
}

\title{
OVERVIEW
}

Acquiring sufficient amounts of high-quality cells remains an impediment to cellbased therapies. Induced pluripotent stem cells (iPSC) may be an unparalleled source, but autologous iPSC likely retain deficiencies requiring correction. We present a strategy for restoring physiological function in genetically deficient iPSC utilizing the low-density lipoprotein receptor (LDLR) deficiency Familial Hypercholesterolemia (FH) as our model. FH fibroblasts were reprogrammed into iPSC using synthetic modified mRNA. FH-iPSC exhibited pluripotency and differentiated toward a hepatic lineage. To restore LDLR endocytosis, FH-iPSC were transfected with a $31 \mathrm{~kb}$ plasmid ( $p E H Z-L D L R-L D L R$ ) containing a wild-type $L D L R$ (FH-iPSC-LDLR) controlled by $10 \mathrm{~kb}$ of upstream genomic DNA as well as Epstein-Barr sequences (EBNA1 and OriP) for episomal retention and replication. After six months of selective culture, $p E H Z-L D L R-L D L R$ was recovered from FH-iPSC-LDLR and transfected into Ldlr-deficient CHO-a7 cells, which then exhibited feedback-controlled LDLR-mediated endocytosis. To quantify endocytosis, FHiPSC \pm LDLR were differentiated into mesenchymal cells (MC), pretreated with excess 
free sterols, Lovastatin, or ethanol (control), and exposed to DiI-LDL. FH-MC-LDLR demonstrated a physiological response, with virtually no DiI-LDL internalization with excess sterols and an $\sim 2$-fold increase in DiI-LDL internalization by Lovastatin compared to FH-MC. These findings demonstrate the feasibility of functionalizing genetically deficient iPSC using episomal plasmids to deliver physiologically responsive transgenes.

\section{INTRODUCTION}

Induced pluripotent stem cells (iPSC) derived from patients are a unique, potential alternative for replacing diseased tissue because of their virtually unlimited supply, differentiation potential, and tolerance by the immune system ${ }^{418}$. However, in the context of treating genetic disorders, autologous iPSC are challenged by their genetic homology with patient DNA, as they may retain functional deficiencies. Such shortcomings must be addressed before these cells attain broad therapeutic value.

Familial Hypercholesterolemia (FH) is one example of a monogenic autosomal dominant disorder that affects low-density lipoprotein cholesterol (LDL) metabolism ${ }^{419}$. In FH, LDL receptor (LDLR) deficiency (attributed to at least one of 1200 documented mutations $^{420,421}$ ) impedes receptor-mediated endocytosis of LDL, resulting in pathologically elevated serum LDL levels. Consequently, the formation of atherosclerotic plaques and cardiovascular disease are both accelerated. Because healthy liver hepatocytes contain the body's highest density of functional LDLR ${ }^{82}$, liver transplant is regarded as the only cure for FH. Yet, the number of available livers is far outstripped by significant demand ${ }^{422}$, while long-term transplant issues such as immunomodulation and transplant rejection remain key hurdles. Further, liver hepatocytes culture poorly ${ }^{423}$, 
complicating efforts such as banking and expansion. For such reasons, generating hepatocytes from patient-specific iPSC (FH-iPSC) may be a possible therapeutic alternative.

We used FH as a model system for interrogating the LDLR functionalization of FH-iPSC. We restored FH-iPSC LDLR-mediated endocytosis using a novel $31 \mathrm{~kb}$ plasmid containing (a) wild-type $L D L R$ physiologically controlled by $10 \mathrm{~kb}$ upstream genomic regulatory control sequence and (b) the minimum number of required EpsteinBarr Virus (EBV) replication and retention sequences (Epstein-Barr Nuclear Association

1 (EBNA1) and origin of plasmid replication $($ OriP $))^{424}$. This large plasmid was transfected into iPSC via electroporation and retained as a stable episome. FH-iPSC were differentiated into hepatocyte-like cells (FH-HLC) and mesenchymal cells (FH-MC) to investigate LDL internalization. Transfected FH-iPSC derivatives demonstrated a significant, physiologically sensitive restoration of LDLR-mediated endocytosis compared to non-transfected controls. The technologies and methods discussed herein represent a unique approach for functionalizing genetically deficient iPSC using episomal plasmids that contain genomic transgene control sequences.

\section{MATERIALS \& METHODS}

Ethics Statement. All animal procedures were conducted in compliance with University of Louisville School of Medicine IACUC-approved protocols and NIH guidelines. All recombinant DNA work was approved by the University of Louisville Institutional Biosafety Committee and performed in accordance with NIH guidelines. 
General Cell Culture. Mouse embryonic fibroblasts (MEF) were cultured in MEF media comprised of DMEM-HG, $2 \mathrm{mM}$ L-glutamine, and 10\% standard fetal bovine serum (FBS; Invitrogen). Chinese hamster ovary wild-type (CHO-WT) and LDLR deficient (CHO-a7) cells (generously provided by Prof. Monty Krieger, Massachusetts Institute of Technology) were cultured in media containing DMEM/F12 (Invitrogen), $2 \mathrm{mM} \mathrm{L}$-glutamine, and $10 \%$ standard FBS. All cells were maintained at $37^{\circ} \mathrm{C}$ and $5 \%$ $\mathrm{CO}_{2}$ and passaged at confluence with $0.05 \%$ Trypsin-EDTA (Invitrogen).

Parent Fibroblast Culture. Dermal fibroblasts from an FH patient (GM01355; Coriell Cell Repositories) were cultured in FH growth medium consisting of MEM (Invitrogen), 15\% HyClone ${ }^{\mathrm{TM}}$ defined FBS (Thermo Fisher Scientific), $2 \mathrm{mM}$ Lglutamine, $0.1 \mathrm{mM}$ Non-Essential Amino Acids (MNEAA), 50 units $/ \mathrm{ml}$ penicillin and 50 $\mu \mathrm{g} / \mathrm{ml}$ streptomycin (Invitrogen). WT IMR90 fibroblasts were cultured in MEF media as described above. All cells were maintained in standard cell culture conditions and passaged with $0.05 \%$ Trypsin-EDTA.

iPSC Generation and Culture. Reprogramming was performed in 6-well tissue culture dishes coated with $0.2 \%$ gelatin (Sigma-Aldrich). On Day -2, Mitomycin-Cinactivated human foreskin fibroblast feeder cells (Nuff; GlobalStem) were plated at a density of $2.5 \times 10^{5}$ cells/well and cultured in Nuff growth media, comprised of DMEMHG, 10\% HyClone defined FBS, 1\% GlutaMAX, 50 units $/ \mathrm{ml}$ penicillin and $50 \mu \mathrm{g} / \mathrm{ml}$ streptomycin (Invitrogen). On Day -1, 2.5x10 $0^{4} \mathrm{FH}$ parent fibroblasts were seeded atop the Nuff feeder layer in FH growth medium. After overnight plating, fibroblasts were transfected daily for 20 days in Pluriton reprogramming medium supplemented with 200 $\mathrm{ng} / \mathrm{ml} \mathrm{B18R}$ interferon inhibitor (Stemgent), the mRNA cocktail, and Lipofectamine- 
RNAiMax Transfection Reagent (Invitrogen) per the manufacturers' instructions. Fresh reprogramming medium was substituted after 4 hours of incubation. Pluripotent colonies (FH-iPSC; TRA-1-60 ${ }^{+}$via staining with StainAlive TRA-1-60 (Stemgent)) were identified and manually passaged from transfected cultures onto Mitomycin C-inactivated (Sigma-Aldrich) MEF every three days. FH-iPSC were cultured with human iPSC medium (DMEM/F12 supplemented with 20\% Knockout Serum Replacement (KSR), 2 mM L-glutamine, $0.1 \mathrm{mM}$ MNEAA (Invitrogen), $0.1 \mathrm{mM} \beta$-mercaptoethanol (SigmaAldrich) and $20 \mathrm{ng} / \mathrm{ml}$ fibroblast growth factor-2 (bFGF; Stemgent)) ${ }^{425}$. For feeder-free culture, FH-iPSC were passaged onto hESC-Qualified Matrigel coated plates (BD Biosciences) and gradually adapted to mTeSR1 media (Stemcell Technologies) by serial transition from $20 \%$ KSR culture media over a 10 -day period with daily media changes. Cells in feeder-free culture were used for parenchymal cell generation before the $10^{\text {th }}$ passage $^{426}$.

FH Cell Characterization. DNA karyotyping and fingerprint analysis (Cell Line Genetics) was completed on FH skin fibroblasts and reprogrammed iPSC (P21) to ensure that both populations were karyotypically normal and maintained identical DNA fingerprints. To verify disease phenotype, FH fibroblasts were compared against wildtype IMR90 fetal lung fibroblasts on the basis of their ability to internalize LDL cholesterol. FH-iPSC pluripotence was then assessed via in vitro spontaneous differentiation and in vivo teratoma assays. In vitro, FH-iPSC were plated on glass slides in differentiation medium (human iPSC medium without bFGF) and differentiated for 20 days. 50\% media changes were performed every other day. After 20 days, we assessed the iPSC for pluripotent marker expression via immunocytochemistry. For in vivo 
teratoma generation, we utilized FH-iPSC that were passaged with $0.05 \%$ Trypsin-EDTA at least five times prior ${ }^{427}$. Growth-Factor Reduced Matrigel (BD Biosciences) was mixed with an FH-iPSC cell pellet to a final concentration of $2.5 \times 10^{4}$ cells $/ \mu 1 ; 200 \mu 1$ was loaded into sterile $1 \mathrm{ml}$ syringes with $18 \mathrm{G}$ needles (BD Biosciences). Control syringes containing only Matrigel were also prepared. iPSC and controls were injected on opposing sides into the subcutaneous dorsum of isoflurane-anesthetized NOD/SCID- $\gamma$ mice (Jackson Laboratories). Injected mixtures were allowed 15 minutes to polymerize before the cessation of anesthesia. At 7 weeks post injection, masses were palpable in the flanks of the animals; at 14 weeks, the teratomas were visualized in situ, excised with gross margins and processed for histological examination.

Verification of Plasmid Functionality. To verify plasmid functionality, Ldlrdeficient CHO-a7 cells were first plated to confluence in a 48-well tissue culture dish. Once confluent, CHO-a7 cells were transfected with a complex of plasmid and Lipofectamine 2000 (Invitrogen) in a $1 \mu \mathrm{g}: 1 \mu \mathrm{l}$ ratio in Opti-MEM (Invitrogen) per manufacturer instructions ${ }^{11}$. Cells were cultured overnight in standard culture conditions. The following day, transfection media was removed and replaced with standard CHOculture media (described above). 24 hours later, selection for transfected CHO-a7 (CHOa7-LDLR) took place via exposure to $750 \mu \mathrm{g} / \mathrm{ml}$ Hygromycin B in CHO culture media. Afterwards, cells were starved overnight in media comprised of DMEM-HG, 1X Lglutamine and 5\% lipoprotein-deficient serum (Alfa Aesar). Starvation media was augmented with $2 \mu \mathrm{M}$ of Lovastatin (EMD Millipore), excess sterols (12 $\mu \mathrm{g} / \mathrm{ml}$ cholesterol and $0.6 \mu \mathrm{g} / \mathrm{ml} 25$-hydroxysterol; Sigma-Aldrich), or ethanol (vehicle control; Sigma-Aldrich) $)^{424}$. The following day, the starvation media was removed and replaced 
with $5 \mu \mathrm{g} / \mathrm{ml}$ DiI-LDL (Alfa Aesar) in DMEM-HG for 4.5 hours, after which the cells were thoroughly washed with di-cationic PBS and imaged using an Olympus IX81 fluorescence microscope.

FH-iPSC Electroporation and Selection. We transfected FH-iPSC with the corrective plasmid using both decayed-exponential and square-wave electroporation modalities. For FH-iPSC used in decayed-exponential electroporation (conducted at $4^{\circ} \mathrm{C}$ ), cells grown in feeder-free culture were pretreated with $10 \mu \mathrm{M}$ of Y-27632 (EMD Millipore) two hours prior to electroporation. The cells were treated with $1 \mathrm{mg} / \mathrm{ml}$ Collagenase Type IV (Invitrogen) for 10 minutes, resuspended in DMEM/F12, and counted. We utilized a minimum of $10^{7}$ cells per electroporation. Cells were pelleted and resuspended in $700 \mu \mathrm{l}$ of ice-cold $\left(4^{\circ} \mathrm{C}\right)$ di-cationic PBS or ES-qualified electroporation buffer (MEB; EMD Millipore). $100 \mu \mathrm{g}$ of plasmid DNA (per $10^{7}$ cells) was Q.S. to $50 \mu 1$ with electroporation buffer. Cell and plasmid solutions were combined; the resulting $750 \mu \mathrm{l}$ volume was added to a pre-chilled $\left(4^{\circ} \mathrm{C}\right) 4 \mathrm{~mm}$ gap cuvette (BTX) and gently mixed by flicking. Cells were electroporated using an ECM 630 Electro Cell Manipulator (BTX; generously provided by Dr. Scott Whittemore, University of Louisville; see Table 4). Electroporated cells were incubated on ice for 5 minutes and suspended in $10 \mathrm{ml}$ DMEM/F12 before pelleting. The pellet was resuspended in mTeSR1 with $10 \mu \mathrm{M}$ Y27632 and plated into 3 Matrigel-coated wells of a 6-well tissue culture dish. For FHiPSC used in square wave electroporation (carried out at room temperature), cells were pretreated with $10 \mu \mathrm{M}$ Y-27632 2 hours prior to electroporation. The cells were harvested via a 10-minute application of $1 \mathrm{mg} / \mathrm{ml}$ Collagenase Type IV and washed twice with Opti-MEM. Cells were placed into microcentrifuge tubes at a ratio of $10^{6}: 90 \mu 1$ 


\begin{tabular}{|c|c|c|c|c|c|c|c|c|c|c|c|}
\hline \multicolumn{12}{|c|}{ BTX 630, Decaying Exponential Electroporator, 4mm Gap Cuvette Parameters } \\
\hline $\begin{array}{c}\text { Cells } \\
(\mathbf{x 1 0})\end{array}$ & $\begin{array}{c}\text { Plasmid } \\
(\mu \mathrm{g})\end{array}$ & $\begin{array}{l}\text { Volt. } \\
\text { (V) }\end{array}$ & $\begin{array}{c}\text { Capac. } \\
(\mu \mathrm{F})\end{array}$ & 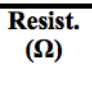 & $\begin{array}{c}\tau \\
(\mathrm{ms})\end{array}$ & $\begin{array}{l}\text { Temp } \\
\left({ }^{\circ} \mathrm{C}\right)\end{array}$ & $\begin{array}{l}\text { Vol } \\
(\mu l)\end{array}$ & Buffer & $\begin{array}{c}\text { Rock } \\
\text { Inhibitor } \\
\text { Pretreatment }\end{array}$ & $\begin{array}{l}\text { Electro. } \\
\text { Survival }\end{array}$ & $\begin{array}{c}\text { Hygro. } \\
\text { Survival }\end{array}$ \\
\hline 10 & 35 & 300 & 960 & 200 & 23.1 & 4 & 300 & MEB & $\mathrm{N}$ & $\mathrm{N}$ & NA \\
\hline 10 & 35 & 320 & 250 & 0 & 8 & 4 & 300 & MEB & $\mathrm{N}$ & $\mathrm{Y}$ & $\mathrm{N}$ \\
\hline 10 & 100 & 300 & 250 & 0 & 6.9 & 4 & 700 & PBS & $\mathrm{Y}$ & $\mathrm{Y}$ & $\mathrm{N}$ \\
\hline 10 & 100 & 300 & 975 & 0 & 16.4 & 4 & 700 & PBS & $\bar{Y}$ & $\mathrm{Y}$ & $\mathrm{Y}$ \\
\hline 10 & 100 & 300 & 975 & 200 & 23.1 & RT & 300 & MEB & $\mathrm{Y}$ & $\mathrm{N}$ & NA \\
\hline 10 & 100 & 300 & 975 & 0 & 18 & 4 & 700 & MEB & $\mathrm{Y}$ & $\mathrm{Y}$ & $\mathrm{Y}$ \\
\hline 3 & 25 & 300 & 975 & 0 & 31.7 & 4 & 350 & MEB & $\mathrm{Y}$ & $\mathrm{N}$ & NA \\
\hline 10 & 25 & 170 & 200 & 1000 & 9 & 4 & 300 & PBS & $\mathrm{N}$ & $\mathrm{Y}$ & $\mathrm{N}$ \\
\hline 10 & 50 & 170 & 200 & 1000 & 10.5 & 4 & 300 & PBS & $\mathrm{N}$ & $\mathrm{Y}$ & $\mathrm{N}$ \\
\hline 10 & 100 & 170 & 200 & 1000 & 11.2 & 4 & 300 & PBS & $\mathrm{N}$ & $\mathrm{Y}$ & $\mathrm{N}$ \\
\hline 10 & 25 & 170 & 250 & 1000 & 12.6 & 4 & 300 & PBS & $\mathrm{N}$ & $\mathrm{Y}$ & $\mathrm{N}$ \\
\hline 10 & 50 & 170 & 250 & 1000 & 12.7 & 4 & 300 & PBS & $\mathrm{N}$ & $\mathrm{Y}$ & $\mathrm{N}$ \\
\hline 10 & 100 & 170 & 250 & 1000 & 13.4 & 4 & 300 & PBS & $\mathrm{N}$ & $\mathrm{Y}$ & $\mathrm{N}$ \\
\hline 10 & 35 & 300 & 960 & 200 & 23.1 & RT & 300 & MEB & $\mathrm{N}$ & $\mathrm{N}$ & NA \\
\hline 10 & 35 & 320 & 250 & 0 & 8 & RT & 300 & MEB & $\mathrm{N}$ & $\mathrm{Y}$ & $\mathrm{N}$ \\
\hline \multicolumn{12}{|c|}{ NEPA-21, Square Wave Electroporator, $2 \mathrm{~mm}$ Gap Cuvette: Poring Pulse Variable Parameters } \\
\hline \multicolumn{2}{|c|}{ Voltage (V) } & \multicolumn{2}{|c|}{ Length (ms) } & \multicolumn{3}{|c|}{$\begin{array}{c}\text { Rock Inhibitor } \\
\text { Pretreatment }\end{array}$} & \multicolumn{2}{|c|}{$\begin{array}{c}\text { Electroporation } \\
\text { Survival }\end{array}$} & \multicolumn{3}{|c|}{ Hygromycin Survival } \\
\hline \multicolumn{2}{|c|}{225} & \multicolumn{2}{|c|}{5} & \multicolumn{3}{|c|}{$\mathrm{Y}$} & \multicolumn{2}{|c|}{$\mathrm{Y}$} & \multicolumn{3}{|c|}{$\mathrm{N}$} \\
\hline \multicolumn{2}{|c|}{275} & \multicolumn{2}{|c|}{2.5} & \multicolumn{3}{|c|}{$\mathrm{Y}$} & \multicolumn{2}{|r|}{$\mathrm{Y}$} & \multicolumn{3}{|c|}{$\mathrm{N}$} \\
\hline \multicolumn{2}{|c|}{275} & \multicolumn{2}{|c|}{5} & \multicolumn{3}{|c|}{$\mathrm{Y}$} & \multicolumn{2}{|r|}{$\mathrm{Y}$} & \multicolumn{3}{|c|}{$\mathrm{N}$} \\
\hline \multicolumn{2}{|c|}{300} & \multicolumn{2}{|c|}{0.5} & \multicolumn{3}{|c|}{$\mathrm{Y}$} & \multicolumn{2}{|r|}{$\mathrm{Y}$} & \multicolumn{3}{|c|}{$\mathrm{Y}$} \\
\hline \multicolumn{2}{|c|}{300} & \multicolumn{2}{|c|}{1} & \multicolumn{3}{|c|}{$\mathrm{Y}$} & \multicolumn{2}{|r|}{$\mathrm{N}$} & & NA & \\
\hline & 00 & & 2.5 & & $\mathrm{Y}$ & & & $\mathrm{N}$ & & NA & \\
\hline
\end{tabular}

Table 4. Greyed rows indicate successful decayed-exponential and square-wave electroporation parameters that yielded surviving, transfected FH-iPSC with pEHZ-

\section{LDLR-LDLR.}


OptiMEM. Plasmid DNA was maintained in a separate tube at $1 \mu \mathrm{g} / \mu \mathrm{l}$ in TE Buffer (Ambion). Recipient 6-well tissue culture dishes were pre-loaded with mTeSR1 and 10 $\mu \mathrm{M}$ Y -27632 and kept at $37^{\circ} \mathrm{C}$. Prior to electroporation, $10 \mu \mathrm{l}$ of plasmid was mixed with $90 \mu \mathrm{l}$ of iPSC (total electroporation volume $=100 \mu \mathrm{l}$ ) and transferred to an electroporation cuvette (NepaGene). The cuvette was gently flicked to ensure adequate mixture. Cells were electroporated using a NEPA21 cell electroporator (NepaGene; generously provided by Dr. Michal Hetman, University of Louisville; parameters are outlined in Table 4). Transfected cells were directly transferred into each well of the 6well tissue culture recipient plate. For both conditions, after electroporation, cells were cultured for 2 days in mTeSR1 before a 5-day selection with $10 \mu \mathrm{g} / \mathrm{mL}$ Hygromycin B and subsequent maintenance in 0.5 to $1 \mu \mathrm{g} / \mathrm{mL}$ Hygromycin $\mathrm{B}$, indicating successful transfection. Media was changed daily.

Hepatocyte-Like Cell Production. FH-iPSC were plated to confluence in Matrigel-pretreated wells of a 6-well tissue culture plate or 6 Permanox chamber slides (Sigma-Aldrich). Each well of the 6-well plate (used for PCR analysis) and each Permanox slide (used for immunocytochemistry) corresponded with one of the six stages of differentiation (including Stage 0, which represented untreated iPSC). To generate HLC, we used a modified version of the protocols and medias proposed by Song et $\mathrm{al}^{428}$. In lieu of Song's Stage 1 media, we utilized the StemDiff definitive endoderm kit (Stem Cell Technologies) per the manufacturer's instructions. Media changes were performed daily and cells were characterized by the end of Stage 5 .

Hepatocyte-Like Cell General Functional Assessment. Indocyanine green (ICG; Sigma-Aldrich; $5 \mathrm{mg} / \mathrm{ml}$ in sterile water) was used to measure overall cell 
metabolic activity. ICG and Stage 5 media were mixed per manufacturer's instructions and applied to FH-HLC for 1 hour. FH-HLC were thoroughly washed with di-cationic PBS to remove residual dye before being imaged on an Olympus IX81 microscope via phase and bright field settings. FH-HLC were then incubated in Stage 5 media overnight before assessment 24 hours later using the same microscope settings. An Oil-Red-O assay was used to evaluate cell lipid accumulation. A different population of FH-HLC was treated with Carnoy's fixative for 2 minutes at RT before being washed with distilled water. Working Oil-Red-O solution (Sigma-Aldrich) was prepared per the manufacturer's instructions and applied to FH-HLC for 30 minutes at RT. Cells were thoroughly washed with distilled water and imaged on an Olympus IX81 microscope with phase and bright field settings.

Imaging of Hepatocyte-Like Cell LDLR-Mediated Endocytosis. FH-HLC derived from transfected FH-iPSC (FH-HLC-LDLR) were starved overnight in 5\% lipoprotein-deficient serum supplemented with $2 \mu \mathrm{M}$ of Lovastatin, excess sterols (12 $\mu \mathrm{g} / \mathrm{ml}$ cholesterol and $0.6 \mu \mathrm{g} / \mathrm{ml} 25$-hydroxysterol), or ethanol (vehicle control). The following day, the starvation media was replaced with $5 \mu \mathrm{g} / \mathrm{mL}$ DiI-LDL in DMEM-HG for 4.5 hours. Cells were thoroughly washed with di-cationic PBS prior to imaging via an Olympus IX81 fluorescence microscope.

Transfected Hepatocyte-Like Cell Qualitative Imaging. FH-HLC derived from transfected FH-iPSC (FH-HLC-LDLR) were grown in 3 wells of a 12-well tissue culture plate. Cells were starved overnight in 5\% lipoprotein-deficient serum supplemented with $2 \mu \mathrm{M}$ of Lovastatin, excess sterols $(12 \mu \mathrm{g} / \mathrm{ml}$ cholesterol and $0.6 \mu \mathrm{g} / \mathrm{ml} 25-$ hydroxysterol), or ethanol (vehicle control). The following day, starvation media was 
removed and replaced with $5 \mu \mathrm{g} / \mathrm{mL}$ DiI-LDL in DMEM high glucose for 4.5 hours. Cells were thoroughly washed with di-cationic PBS prior to imaging via an Olympus IX81 fluorescence microscope.

Hepatocyte-Like Cell In Vivo Assessment. Human SVF (hSVF) was grown to confluence in vitro in a gelatin-coated T75 flask while FH-HLC were grown to confluence through end of Stage 2. On the day of implantation, both hSVF and HLC were prepared using $0.05 \%$ Trypsin-EDTA. Constructs were prepared using $10^{6} / \mathrm{ml} \mathrm{HLC}$ $\pm 10^{6} / \mathrm{ml}$ of SVF in a master mixture of $3 \mathrm{mg} / \mathrm{ml}$ Collagen I (comprised of Rat Tail Collagen I (BD Biosciences), 4X DMEM (Invitrogen; made from 10X powdered concentrate), Sterile Water, and 0.1N NaOH (Ricca Chemical Company)). $250 \mu 1$ of the cellular mixture was pipetted into each well of a 48 -well plate and maintained at $37^{\circ} \mathrm{C}$ to polymerize for 30 minutes. Afterwards, the constructs were immersed in a mixture of 50\% Stage 2 HLC differentiation medium and 50\% Human Complete hSVF culture media (comprised of Medium 199 (Invitrogen), ECGS (derived in-house), HEPES, 1X Lglutamine (Invitrogen), and 10\% heat-inactivated FBS (Thermo Fisher Scientific)). Constructs were implanted in the subcutaneous dorsa of isoflurane-anesthetized $\operatorname{Rag} 1^{-/-} \mathrm{x}$ $\mathrm{LDLR}^{-/}$double knockout mice (generously provided by Dr. Catherine Reardon-Alulis, University of Chicago). Animals were sacrificed at 2 weeks, at which point the implanted constructs were extricated and fixed in 4\% PFA for $1 \mathrm{~h}$. Constructs were then permeabilized in $0.5 \%$ Triton X-100 (MP Biomedicals) for 20m and blocked in 5\% Goat Serum (Invitrogen) for $1 \mathrm{~h}$. The explants were then incubated overnight in Albumin (MP Biomedicals) and UEA-1 biotin (Vector Labs) at 1:200 in 5\% Goat Serum at $4{ }^{\circ} \mathrm{C}$. The following day, the constructs were washed in di-cation-free PBS and exposed to Goat- 
anti-Rabbit 488 (Thermo Fisher Scientific; for Albumin) and Streptavidin-Cy5 (Invitrogen; for UEA-1 biotin) at 1:1000 for $1 \mathrm{~h}$. Constructs were labeled with DAPI at 1:10000 for 10 minutes prior to confocal fluorescence microscopy imaging. All animal procedures were conducted in accordance with University of Louisville School of Medicine IACUC regulations.

Plasmid Rescue and Restriction Digest. Plasmid rescue was performed on transfected FH-iPSC-LDLR using methods described by Lufino and colleagues ${ }^{429}$. The rescued plasmid was characterized using AgeI-HF restriction enzyme (New England BioLabs) per manufacturer instructions. Restriction products were run on a $0.4 \%$ agarose gel at $20 \mathrm{~V}$ for 16 hours. Gels were analyzed via a Typhoon 9400 variable mode imager (GE Healthcare Bio-Sciences). After confirming plasmid isolation, the rescued plasmid was also re-transfected into $L d l r$-deficient $\mathrm{CHO}-\mathrm{a} 7$ cells (generously provided by Prof. Monty Krieger, Massachusetts Institute of Technology) via Lipofectamine 2000 to demonstrate non-integration and preservation of plasmid functionality after rescue.

FH Mesenchymal Cell Generation and Culture. Mesenchymal cells were generated as previously described ${ }^{425}$. FH-iPSC \pm LDLR were passaged onto Matrigelcoated plates using Gentle Cell Dissociation Buffer (Stem Cell Technologies) and introduced to $100 \%$ mTeSR 1 in a graded manner. One day after passaging, transfected cultures received $1 \mu \mathrm{g} / \mathrm{ml}$ Hygromycin B to maintain selective pressure. Once confluent and in $100 \%$ mTeSR1, the media was changed to EGM2-MV (Lonza) for 20 days for differentiation into FH-MC. Once fully differentiated, FH-MC were passaged and used in LDL internalization assays. 
Quantification of $\boldsymbol{p E H Z}-\boldsymbol{L D L R}-\boldsymbol{L D L R}$ Activity. FH-MC \pm LDLR were plated in gelatin-coated wells of a 24-well tissue culture plate. We utilized an established protocol $^{430}$, but used fluorescent DiI-LDL (Alfa Aesar) in place of ${ }^{125}$ Iodinated-LDL. Specific receptor-mediated internalization was determined by subtracting the internalization of [DiI-LDL +50 -fold excess of unlabeled LDL (Alfa Aesar)] from the internalization quantified with DiI-LDL alone. Cells were then lysed with $250 \mu 10.1 \mathrm{~N}$ $\mathrm{NaOH}$ (Ricca Chemical Company). Fluorescence was quantified using a 96-well plate reader (BioTek), with the excitation and emission set to $514 \mathrm{~nm}$ and $550 \mathrm{~nm}$, respectively. Fluorescence units were normalized to total protein content, which was determined via a DC Protein Assay kit (Bio-Rad).

Polymerase Chain Reaction (PCR) and Gel Electrophoresis. Differentiating FH-iPSC were lysed at the end of each stage using $350 \mu \mathrm{l}$ of $0.1 \% \beta$-mercaptoethanol in RLT Buffer (Qiagen). Lysates were purified using Qiashredder and RNeasy kits (Qiagen) according to the manufacturer's instructions. RNA was quantified using a NanoDrop 1000 Spectrophotometer (generously provided by Ronald Gregg, University of Louisville). cDNA was generated from purified RNA via a SuperScript II Reverse Transcriptase kit (Invitrogen). $200 \mathrm{ng}$ of stage-specific cDNA was added to $45 \mu \mathrm{l}$ of PCR SuperMix (Invitrogen) and $1 \mu 1$ each of $10 \mu \mathrm{M}$ forward and reverse primers (Integrated DNA Technologies; primer sequences can be found in Table 5). PCR was performed for 25 cycles, with denaturation at $95^{\circ} \mathrm{C}$, annealing at $58^{\circ} \mathrm{C}$, and extension at $72^{\circ} \mathrm{C}$. Amplicons were assessed via 3\% agarose gels (Bio-Rad). $10 \mu \mathrm{l}$ of amplicons were combined with $2 \mu$ of TrackIt ${ }^{\mathrm{TM}}$ Cyan/Yellow Loading Buffer (Invitrogen) and $2 \mu \mathrm{l}$ of 


\begin{tabular}{|c|c|c|c|}
\hline Gene Name & $\begin{array}{c}\text { Primer Sequence } \\
\left(5^{\prime} \rightarrow 3^{\prime}\right)\end{array}$ & Size (bp) & $\begin{array}{c}\text { Primer- } \\
\text { BLAST } \\
\text { Annealing } \\
\text { Temperature }\end{array}$ \\
\hline POU5F1 & $\begin{array}{c}\text { Forward: } \\
\text { TGATCCTCGGACCTGGCTAA } \\
\text { Reverse: } \\
\text { AACCACACTCGGACCACATC }\end{array}$ & 744 & $\begin{array}{l}\text { Forward: } \\
60.03^{\circ} \mathrm{C} \\
\text { Reverse: } \\
59.97^{\circ} \mathrm{C}\end{array}$ \\
\hline SOX17 & $\begin{array}{c}\text { Forward: } \\
\text { CCGCGGTATATTACTGCAACTA } \\
\text { Reverse: } \\
\text { CACCCAGGACAACATTTCTTTG }\end{array}$ & 371 & $\begin{array}{l}\text { Forward: } \\
58.1^{\circ} \mathrm{C} \\
\text { Reverse: } \\
58.02^{\circ} \mathrm{C}\end{array}$ \\
\hline HNF4a & $\begin{array}{c}\text { Forward: } \\
\text { CCACGGGCAAACACTACGG } \\
\text { Reverse: } \\
\text { GGCAGGCTGCTGTCCTCAT }\end{array}$ & 250 & $\begin{array}{l}\text { Forward: } \\
61.32^{\circ} \mathrm{C} \\
\text { Reverse: } \\
62.34^{\circ} \mathrm{C}\end{array}$ \\
\hline AFP & $\begin{array}{c}\text { Forward: } \\
\text { GCTGACCTGGCTACCATATTT } \\
\text { Reverse: } \\
\text { GGGATGCCTTCTTGCTATCTC }\end{array}$ & 384 & $\begin{array}{l}\text { Forward: } \\
57.8^{\circ} \mathrm{C} \\
\text { Reverse: } \\
58.2^{\circ} \mathrm{C}\end{array}$ \\
\hline ALB & $\begin{array}{c}\text { Forward: } \\
\text { GGTGTTGATTGCCTTTGCTC } \\
\text { Reverse: } \\
\text { CCCTTCATCCCGAAGTTCAT }\end{array}$ & 502 & $\begin{array}{l}\text { Forward: } \\
57.93^{\circ} \mathrm{C} \\
\text { Reverse: } \\
57.28^{\circ} \mathrm{C}\end{array}$ \\
\hline NANOG & $\begin{array}{c}\text { Forward: } \\
\text { TCTGCTGGACTGAGCTGGTT } \\
\text { Reverse: } \\
\text { GTGCACCAGGTCTGAGTGTT }\end{array}$ & 866 & $\begin{array}{l}\text { Forward: } \\
61.12^{\circ} \mathrm{C} \\
\text { Reverse: } \\
60.18^{\circ} \mathrm{C}\end{array}$ \\
\hline$\beta$-ACTIN & $\begin{array}{c}\text { Forward: } \\
\text { CTGTGGCATCCACGAAACTA } \\
\text { Reverse: } \\
\text { AGTACTTGCGCTCAGGAGGA }\end{array}$ & 200 & $\begin{array}{l}\text { Forward: } \\
54.94^{\circ} \mathrm{C} \\
\text { Reverse: } \\
58.03^{\circ} \mathrm{C}\end{array}$ \\
\hline GAPDH & $\begin{array}{c}\text { Forward: } \\
\text { AATCCCATCACCATCTTCC } \\
\text { Reverse: } \\
\text { CATCACGCCACAGTTTCC }\end{array}$ & 382 & $\begin{array}{l}\text { Forward: } \\
54.14^{\circ} \mathrm{C} \\
\text { Reverse: } \\
56.43^{\circ} \mathrm{C}\end{array}$ \\
\hline
\end{tabular}

Table 5. A listing of the primers used for RT-PCR. 
SYBR Green 1 nucleic acid stain (Lonza). Gels were run at $70 \mathrm{~V}$ for 2.5 hours and imaged via a Typhoon 9400 variable mode imager. Gel bands were plot profiled and normalized to GAPDH via densitometry analysis with Image $\mathbf{J}$ software (US National Institutes of Health).

Immunocytochemistry. For immunostaining, cells were fixed in $4 \%$ paraformaldehyde (PFA; Electron Microscopy Sciences) for 15 minutes at RT and washed with di-cation PBS. For intracellular iPSC staining, cells were permeabilized with $0.1 \%$ Triton X-100 (Sigma-Aldrich) and 1\% Polyvinylpyrrolidone (Sigma-Aldrich), for iPSC analysis, in a PBS blocking solution containing 4\% normal goat serum (SigmaAldrich). For extracellular staining, cells were blocked in di-cationic PBS containing 4\% goat serum. Primary antibody solutions (1:200; Table 6) were applied for 1 hour at RT and washed cells with di-cation PBS. Secondary antibody solutions (1:500; Table 6) were applied for 1 hour at RT. Cells were treated with ProLong ${ }^{\circledR}$ Gold Antifade Reagent containing DAPI nuclear stain (Invitrogen). Images were taken using an Olympus IX81 fluorescence microscope using SlideBook Software (Intelligent Imaging Innovations, Inc., Denver, CO). To assess FH-iPSC differentiation into FH-HLC, cells were fixed and washed at the end of each stage as described above. After permeabilization with $0.5 \%$ Triton X-100 for 10 minutes, cells were blocked with 5\% goat serum for 1 hour before being exposed to primary antibody overnight and secondary antibody the following day.

Histology. Explanted teratomas were fixed in paraffin (Leica Biosystems) and sectioned via microtome. Sections were de-paraffinized with xylene (Thermo Fisher Scientific) for 10 minutes and sequentially hydrated with decreasing proportions of ethanol in 2-minute increments. Sections were then immersed in water and exposed to 


\begin{tabular}{|c|c|}
\hline PRIMARY ANTIBODY (1:200) & MANUFACTURER \& LOCATION \\
\hline OCT4 & R\&D Systems, Minneapolis, MN \\
\hline SOX2 & R\&D Systems \\
\hline SSEA4 & $\begin{array}{c}\text { Developmental Studies Hybridoma Bank, } \\
\text { Iowa City, IA }\end{array}$ \\
\hline TRA-1-60 & EMD Millipore, Billerica, MA \\
\hline StainAlive $^{\mathrm{TM}}$ TRA-1-60 DyLight $\left.^{\mathrm{TM}} 488\right)$ & Stemgent, Cambridge, MA \\
\hline TRA-1-81 & EMD Millipore \\
\hline BII-TUBULIN & Neuromics, Edina, MN \\
\hline$\alpha-$ SMOOTH MUSCLE ACTIN & Santa Cruz Biotechnology, Dallas, TX \\
\hline VIMENTIN & BD Bioscience, San Jose, CA \\
\hline
\end{tabular}

\begin{tabular}{|c|c|}
\hline FLUOROPHORE (1:500) & MANUFACTURER \& LOCATION \\
\hline Alexa Fluor® 405 & Invitrogen, Grand Island, NY \\
\hline Alexa Fluor® 594 & Invitrogen \\
\hline
\end{tabular}

End-of-stage visual assessments

\begin{tabular}{|c|c|}
\hline PRIMARY ANTIBODY (1:200) & MANUFACTURER \& LOCATION \\
\hline OCT4 & Santa Cruz Biotechnology \\
\hline SOX17 & R\&D Systems \\
\hline GATA4 & Santa Cruz Biotechnology \\
\hline HNF3 $\beta$ & Santa Cruz Biotechnology \\
\hline AFP (ZSA06) & Invitrogen \\
\hline Albumin & MP Biomedicals, Santa Ana, CA \\
\hline
\end{tabular}

\begin{tabular}{|c|c|}
\hline SECONDARY ANTIBODY (1:1000) & MANUFACTURER \& LOCATION \\
\hline Goat anti-Mouse 488 & Thermo Scientific, Waltham, MA \\
\hline Goat anti-Rabbit 488 & Thermo Scientific \\
\hline Rabbit anti-Goat 594 & Invitrogen \\
\hline
\end{tabular}

Table 6. Primary antibodies were incubated at 1:200, Fluorophores at 1:500, and secondary antibodies at 1:1000. 
Gill's Hematoxylin (Leica Biosystems) for 10 minutes. Water was applied to the sections for 1 minute; sections were then dipped once each in acid ethanol (comprised of $3 \mathrm{ml}$ $37 \% \mathrm{HCl}$ (Thermo Fisher Scientific) and $300 \mathrm{ml} 70 \% \mathrm{EtOH}$ ) and water before 15 immersions in ammonia water $(0.9 \mathrm{ml} 30 \%$ Ammonium Hydroxide; Sigma Aldrich) and exposure to running water for 20 minutes. Working Eosin (Leica Biosystems) was applied to sections for 5 minutes before sequential dehydration with increasing proportions of ethanol in 2-minute increments. After two more 2-minute immersions in xylene, sections were mounted with coverslips and imaged via phase microscopy.

Statistical Analysis. Experiments were conducted in triplicate with replicated data points per experiment. Values are presented as means \pm S.E.M. Significance was determined using a Student's $t$-test. Treatment significance was verified by one-way ANOVA using SigmaPlot (Systat Software).

\section{RESULTS}

FH-iPSC can be generated from FH fibroblasts. Skin fibroblasts from a patient with clinically homozygous FH (Coriell Cell Repositories; \#GM01355) were examined to verify their inherent LDLR dysfunction using an LDL internalization assay (Figure 21). After pretreatment with Lovastatin, excess free sterols, and ethanol (vehicle control) in lipoprotein-deficient serum overnight, the cells were exposed to DiI-LDL the following day and found to be impaired in their ability to internalize LDL when compared against wild-type IMR90 fetal lung fibroblasts. Because concerns with iPSC generation include (a) genomic integration of exogenous reprogramming factors and (b) unwanted spontaneous transcriptional activation post genomic integration, we sought to utilize 


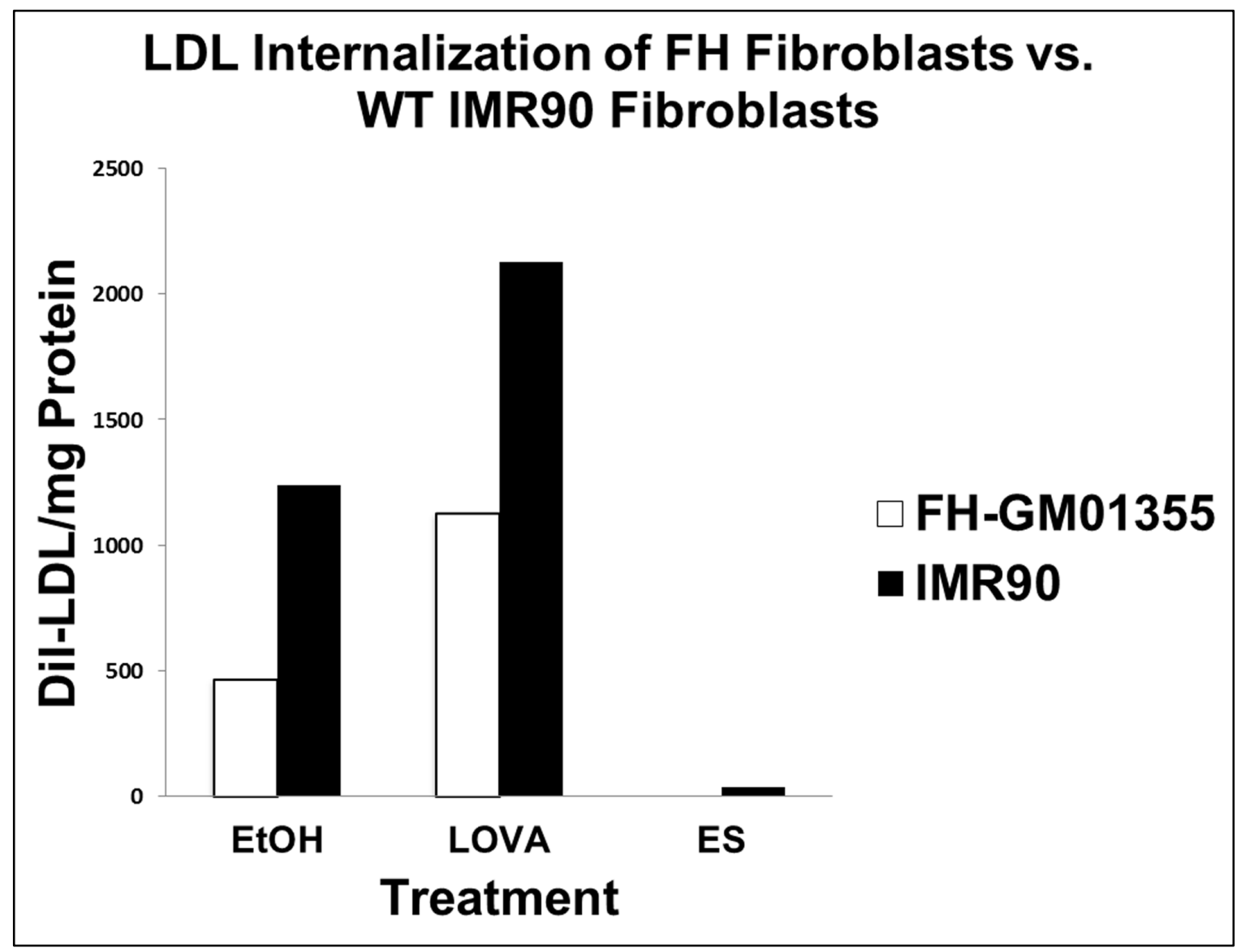

Figure 21. Upon receipt, source FH fibroblasts (GM03155) were compared to wild-type IMR90 fetal lung fibroblasts to determine their ability to internalize fluorescent DiI-LDL after pre-treatment with Lovastatin, excess free sterols, or ethanol (vehicle control) in lipoprotein-deficient media. Reduced internalization of fluorescently labeled LDL indicated a functional reduction in the capacity for receptor-mediated endocytosis. 
mRNA-based reprogramming technologies. FH patient fibroblasts were obtained from Coriell Cell Repositories (\#GM01355; Figure 22A) and transfected with synthetic modified mRNA ${ }^{431}$ (modRNA) (encoding OCT4, SOX2, KLF4, cMYC and LIN28 ${ }^{432}$ ). Within 20 days of continuous transfection, the fibroblasts gradually attained a compacted morphology broadly characteristic of iPSC, with a calculated reprogramming efficiency of $0.012 \%$ (Figure 22B). Live-cell immunolabeling with the extracellular pluripotence marker TRA-1-60 (Figure 22C) suggested successful iPSC reprogramming.

Over the next several days, we observed colonies that possessed increasingly defined borders and cells with high nuclear-to-cytoplasmic ratios and prominent nucleoli. These cell clusters were positive for the intracellular pluripotent markers OCT4 (Figure 22D) and SOX2

(Figure 22E), which co-localized (Figure 22F), giving visual credence to the fact that the source fibroblasts were fully reprogrammed into iPSC $^{433}$. Additionally, the extracellular surface pluripotent markers SSEA4 (Figure 22G), TRA-1-60 (Figure 22H), and TRA-1-81 (Figure 22I) were also visualized. The expression of these intracellular and extracellular pluripotent markers coupled with the aforementioned hPSC-specific morphologic features suggested a successful development of FH patient-specific iPSC from source fibroblasts. To further characterize their lineage and normality, FH fibroblasts and FH-iPSC were subjected to karyotypic analysis (Figure 23) and DNA fingerprinting (not shown), confirming genetic homology between the two cell populations. This validated our production of autologous iPSC and ruled out any experimentally induced genetic abnormalities or variations. 


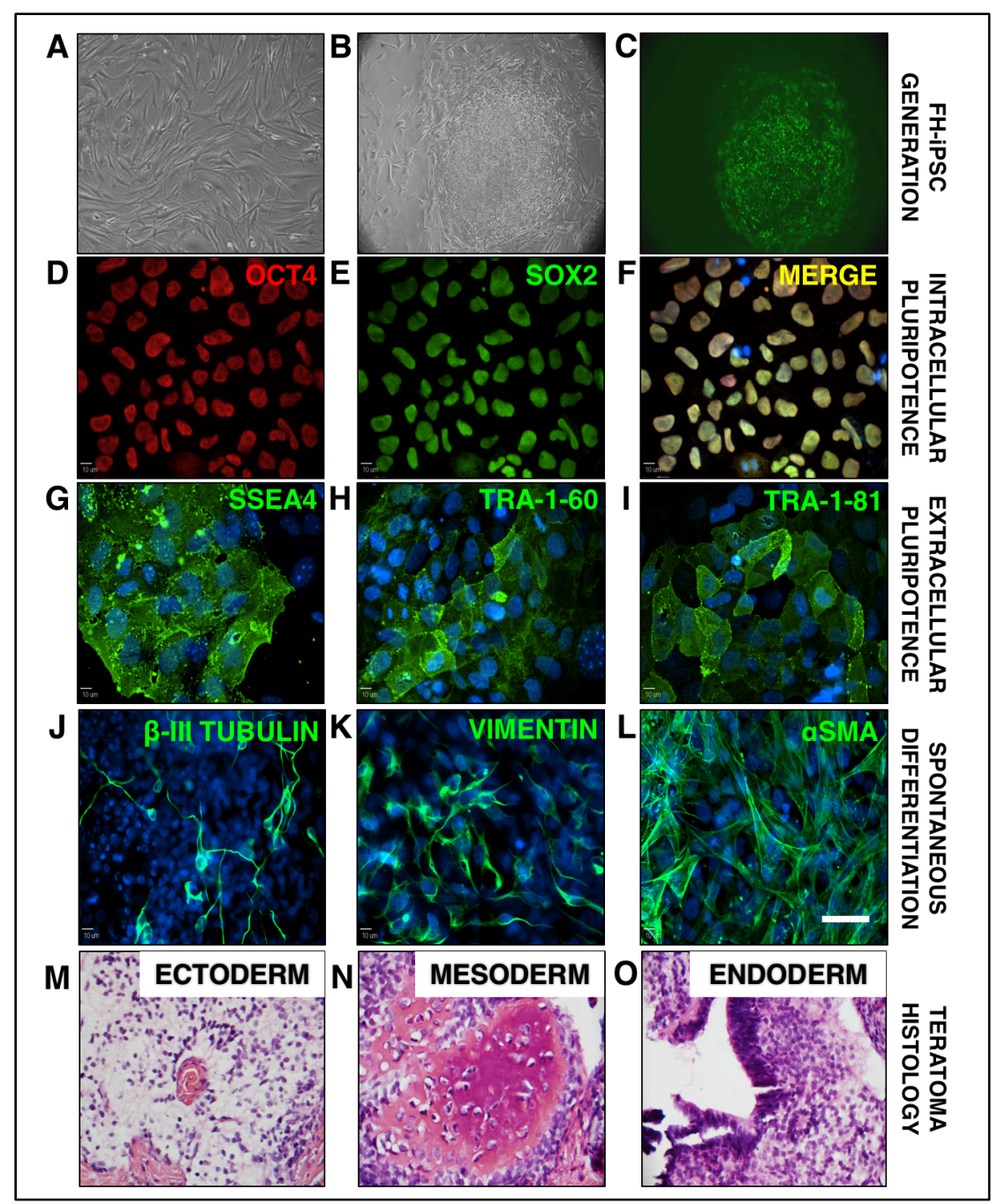

Figure 22. (A) FH parent fibroblasts attained a compacted morphology characteristic of (B) iPSC, which were positive for (C) the pluripotent marker TRA-1-60 during live imaging. FH-iPSC were positive for (D) Oct4 and (E) Sox2, which (F) co-localized to the cell nucleus. FH-iPSC were also positive for the pluripotence markers (G) SSEA4, (H) TRA-1-60, and (I) TRA-1-81. Spontaneous in vitro differentiation yielded cells positive for (J) $\beta$-III TUBULIN (ectoderm), (K) VIMENTIN (endoderm), and (L) $\alpha$-Smooth Muscle Actin (aSMA, mesoderm) (Scale bar $=10 \mu \mathrm{m})$. In vivo differentiation of implanted FH-iPSC yielded teratomas positive for (M) ectoderm, (N) mesoderm, and (O) endoderm. 
We next assessed FH-iPSC pluripotency by spontaneous differentiation in vitro and teratoma formation in vivo. After 20 days of culture without supplemental bFGF, cells expressed the extracellular markers $\beta$-III TUBULIN (ectoderm; Figure 22J), VIMENTIN (endoderm; Figure 22K) and $\alpha$-SMOOTH MUSCLE ACTIN ( $\alpha$-SMA; mesoderm; Figure 22L). For in vivo testing, undifferentiated FH-iPSC were combined with Matrigel and injected into the right subcutaneous dorsum of NOD/SCID- $\gamma^{\text {null }}$ immunocompromised mice, while the left dorsum was injected with Matrigel alone as a negative control. Injected FH-iPSC yielded teratomas with evidence of solid tissue development and displayed a vasculature that anastomosed with that of the host. Histologic assessment of the solid tissue showed clusters of neuroepithelial cells (ectoderm; Figure 22M), regions of cartilage and immature bone formation (mesoderm; Figure 22N), and organized ciliated columnar epithelium (endoderm; Figure 22O). Expectedly, the Matrigel negative control yielded no teratomas (data not shown). This data demonstrates the utility of transient, non-integrating mRNA reprogramming vector systems in generating pluripotent, patient-specific iPSC. The FH-iPSC pluripotent potential was assessed via in vitro spontaneous differentiation and in vivo teratoma formation, with both assays demonstrating formation of the three germ layers (endoderm, mesoderm, and ectoderm). Karyotyping (Figure 23) and DNA fingerprinting (not shown) (a) revealed no reprogramming-attributed chromosomal abnormalities and (b) demonstrated homology between the FH-iPSC and their source fibroblasts. These results demonstrate that the GM01355 FH fibroblasts were successfully reprogrammed into pluripotent FH-iPSC. 


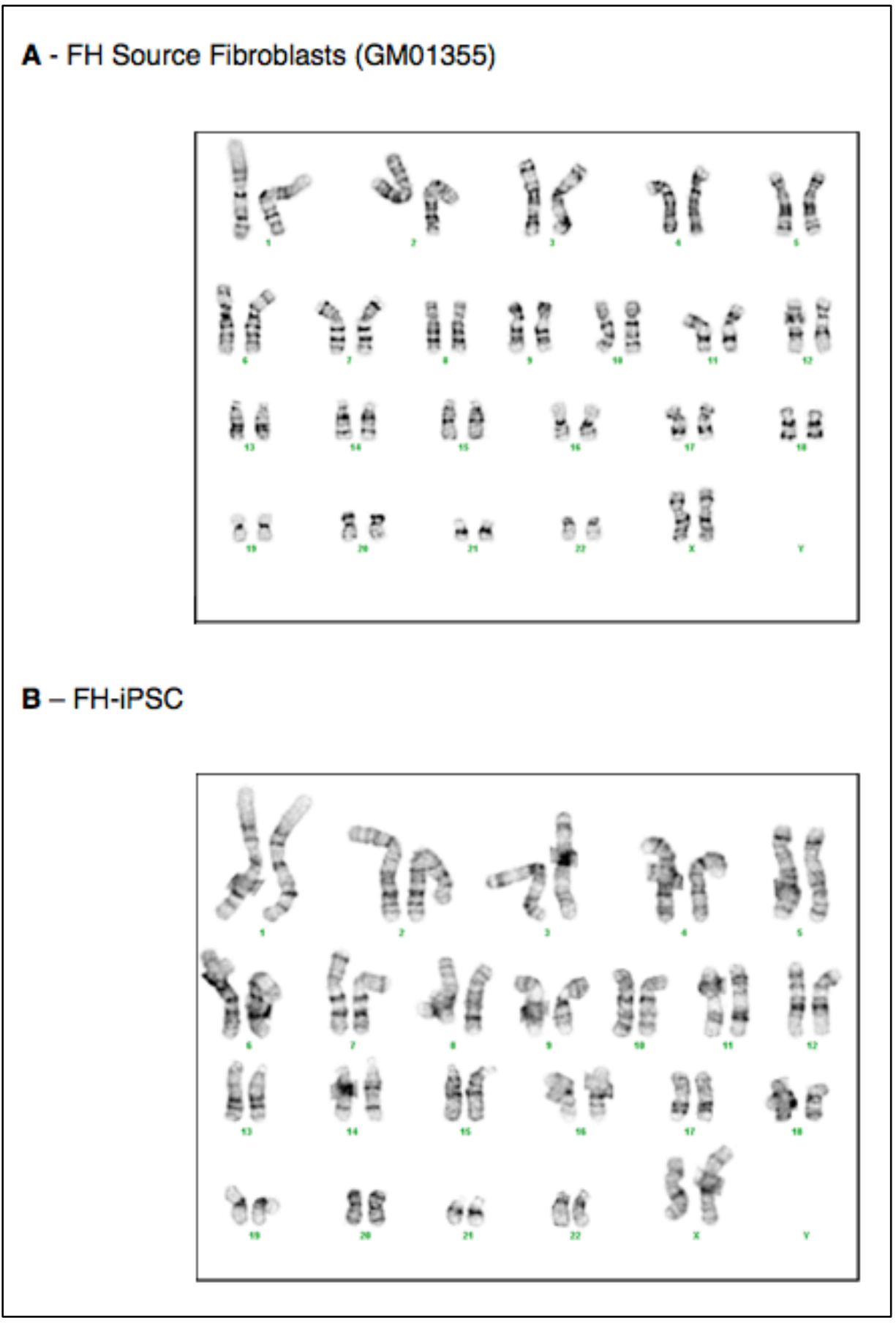

Figure 23. (A) FH parent fibroblasts (GM01355) were tested to determine their karyotype. (B) After reprogramming, FH-iPSC karyotype was also determined. The karyotype of the starting population and reprogrammed daughter cells were both normal. 
FH-iPSC can differentiate towards a hepatic lineage. Since FH patients benefit from a variety of interventions principally targeting hepatocytes, we wanted to determine if FH-iPSC could differentiate towards a hepatic lineage using a modified version of an established hepatic differentiation protocol that mimics developmental embryologic cues (Figure 24A) $)^{428}$. At the end of each stage, we conducted RT-PCR (Figure 24B) and immunocytochemistry (Figure 24C) to follow transcript and protein expression, respectively, throughout the spectrum of HLC derivation. Being undifferentiated, the FHiPSC at Stage 0 expressed OCT4 transcripts and protein that rapidly decreased to undetectable levels in the subsequent stages. In stages 1 and 2, marked cell- specification into definitive endoderm and hepatic-defined lineages was evident from the nuclear expression of SOX17, HNF3 $\beta / 4 \alpha$, and GATA4. Starting in Stage 2, cells were positive for the hepatic markers Alpha-fetoprotein (AFP) and Albumin, which were initially observed as cytoplasmic clumps before attaining a more uniform distribution by Stages 3 and 4. By the end of Stage 5, AFP and Albumin transcript and protein expression predominated, indicating the generation of FH-HLC. 


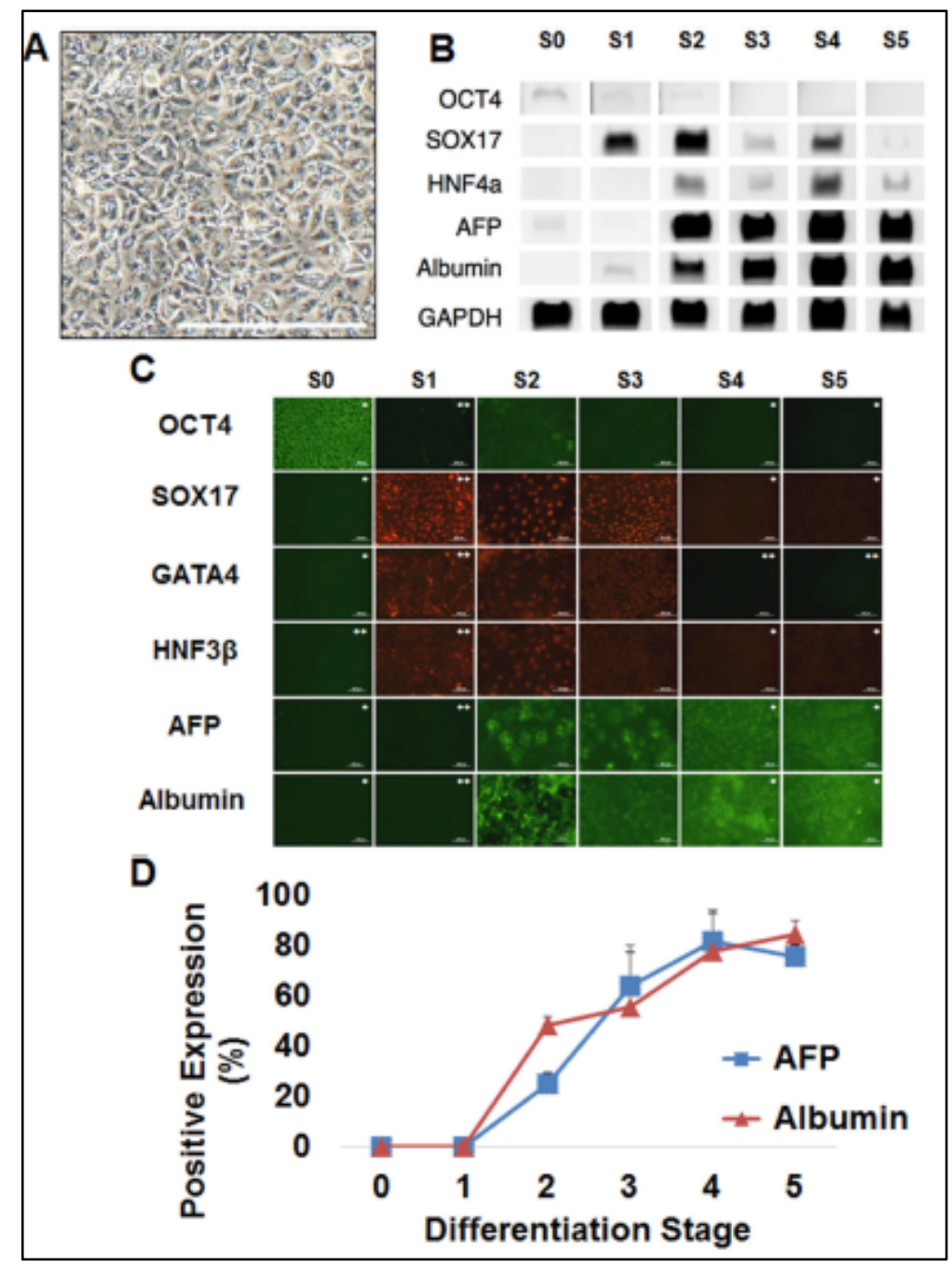

Figure 24. (A) Phase microscopy demonstrates hepatocyte-like cell (HLC) morphology at the end of the five-stage differentiation process (Scale bar $=500 \mu \mathrm{m})$. Representative gel electrophoresis image of PCR transcripts at the end of each differentiation stage. GAPDH was used as a loading control. (C) Immunocytochemistry at the end of each differentiation stage demonstrates a progressive shift in protein expression towards a hepatic phenotype (Scale bars $=50 \mu \mathrm{m}, * 100 \mu \mathrm{m}$, and $* * 200 \mu \mathrm{m}$; images of varying magnifications were chosen for clarity). (D) Quantification of AFP (blue)- and Albumin (red)-positive cells as a fraction of the total number of cells (DAPI), shown for each differentiation stage as mean \pm S.E.M. 
Semi-quantitative PCR densitometry conducted at the end of each stage depicted a progressive change in gene expression, from pluripotence to markers associated with the hepatic lineage (Figure 25). The number of cells expressing AFP and Albumin at each stage was calculated as a percentage of all DAPI-labeled cells (Figure 24D; mean \pm S.E.M.). We noticed the following pattern of AFP expression over the course of the differentiation - Stage 0: 0\%, Stage 1: $0 \%$, Stage 2: $24.74 \pm 4.73 \%$, Stage 3: $63.84 \pm$ 13.25\%, Stage 4: $81.83 \pm 11.99 \%$, and Stage 5: $75.71 \pm 4.08 \%$. We witnessed a similar pattern of Albumin expression as well - Stage 0: 0\%, Stage 1: 0\%, Stage 2: $48.49 \pm$ 3.31\%, Stage 3: $55.3 \pm 24.69 \%$, Stage 4: $78.03 \pm 14.92 \%$, and Stage 5: $84.26 \pm 5.73 \%$. Thus, with respect to differentiation efficiency, most of the cells were positive for the two primary hepatic markers by the end of Stage 5 differentiation. During each round of FHHLC production, we noticed the presence of differentiating cells that took on an increasingly granular, vacuolar appearance (especially present at Stage 3) that looked lipid-like - an observation consistent with that of Fattahi and colleagues ${ }^{434}$. To determine if these vacuoles were in fact accumulating lipids, we subjected the FH-HLC to Oil-Red$\mathrm{O}$ staining and subsequently confirmed the presence of numerous lipid droplets (Figure 26A), suggesting that these cells are not impaired in their ability to store lipids, but perhaps only in their ability to bind and internalize LDL. This corresponds with what is seen clinically, as FH patients often exhibit normal liver function tests ${ }^{435}$. To determine if FH-HLC demonstrated any outward sign of maturation, the cells were treated with Indocyanine green (ICG), a clinically relevant dye that is metabolized and cleared by specific mature cell types including hepatocytes ${ }^{436}$. Though ICG was cleared, this observation was a relatively rare event, suggesting that the 

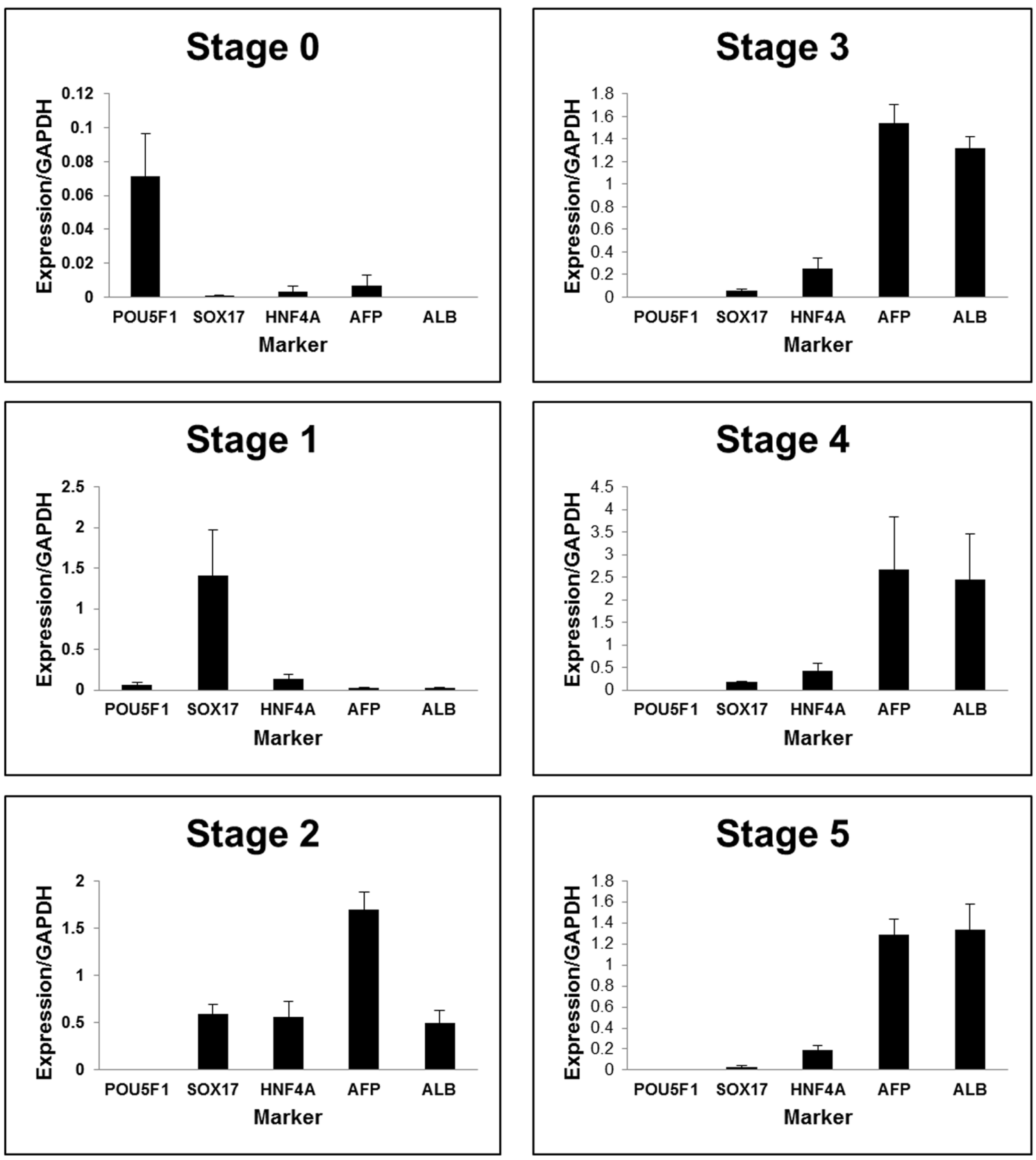

Figure 25. Densitometry quantification of transcript expression was performed for each gene at the end of the differentiation stages. Transcripts were normalized to the GAPDH loading control (shown as mean \pm S.E.M). 


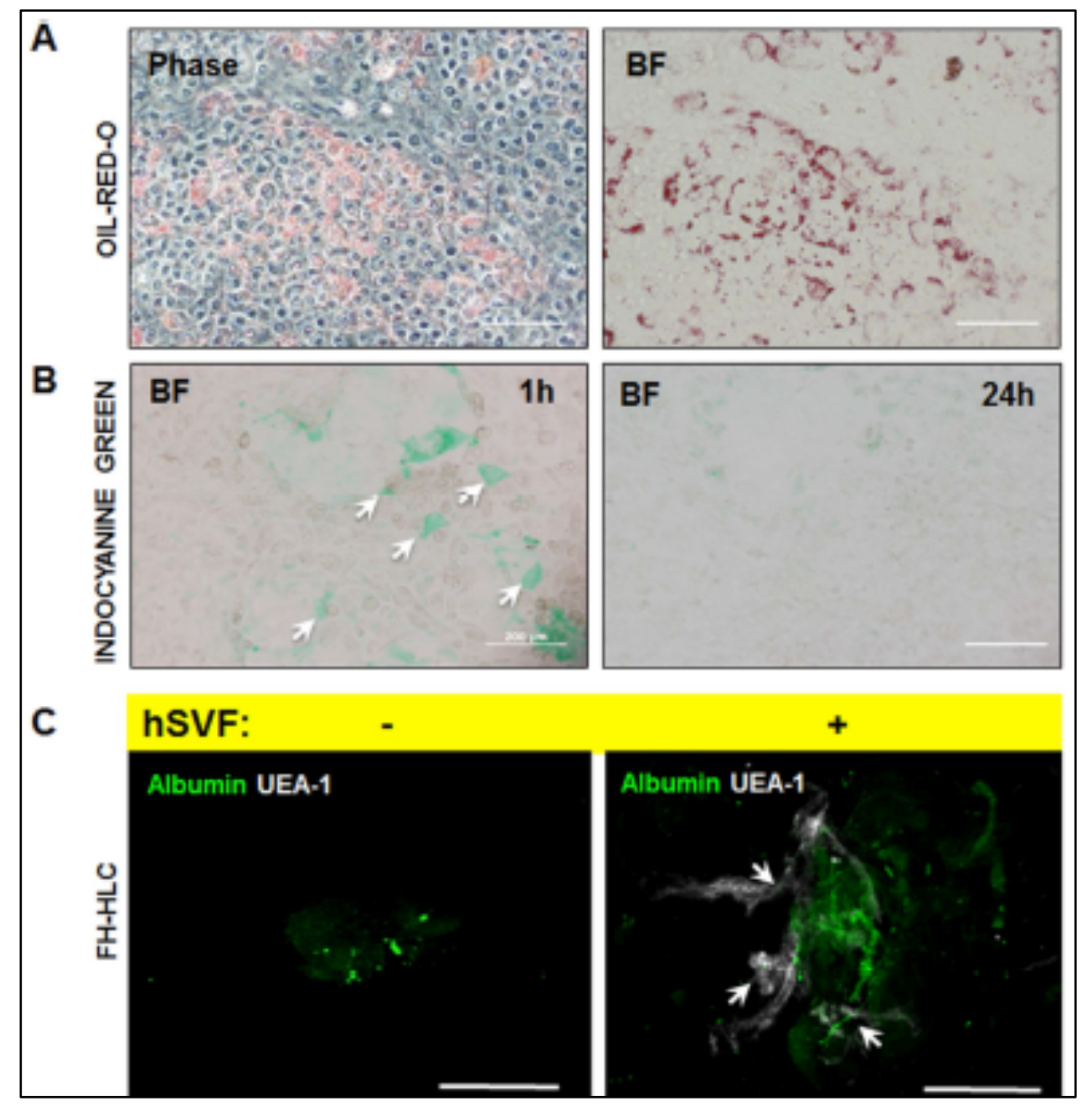

Figure 26. (A) FH-HLC were stained with Oil-Red-O to visualize lipid accumulation with phase and bright field (BF) microscopy. (B) FH-HLC cellular uptake (white arrows) of Indocyanine Green (ICG) was imaged with BF microscopy after 1 hour of treatment. The same site was also assessed for ICG clearance 24 hours later. (Scale bar $=200 \mu \mathrm{m})$. (C) FH-HLC demonstrate survival and engraftment in vivo in Ragl ${ }^{-/} \times \mathrm{LDLR}^{-/}$doubleKO mice when implanted with adipose-derived stromal and vascular support cells (white arrows indicate network formation). FH-HLC without stromal support failed to survive or express Albumin after the two-week implant period. (Scale bar $=50 \mu \mathrm{m})$. 
differentiation protocol yielded a predominantly immature hepatic population (Figure 26B). Still, these results indicate that FH-iPSC can differentiate down a hepatic lineage into FH-HLC. We then asked if FH-HLC could engraft in vivo (Figure 26C). We previously demonstrated the utility of adipose-derived stromal vascular fraction cells (SVF) in providing vital stromal and vascular support ${ }^{437}$ to implanted parenchymal hepatocytes within a liver-tissue mimic ${ }^{438}$. Consequently, we combined human SVF with FH-HLC in collagen-I constructs and implanted them subcutaneously in Rag $1^{-/} \mathrm{x} \mathrm{LDLR}^{-}$ I- double-knockout mice for 2 weeks. We labeled the explanted constructs with Albumin488 and UEA-1-Cy5 (binds to human endothelium). Fluorescence confocal microscopy showed that FH-HLC had engrafted and expressed Albumin in the presence of SVF, whereas FH-HLC did not survive in the absence of SVF. Together, these findings suggest that FH-iPSC can differentiate down a hepatic lineage and, given the proper stromal and vascular support, survive and engraft in vivo.

Verification of $p E H Z-L D L R-L D L R$ functionality. To verify the functionality of pEHZ-LDLR-LDLR, we utilized CHO-a7, an Ldlr-deficient cell line ${ }^{424,439}$ (CHO-a7LDLR) (Figure 27). Transfected $\mathrm{CHO}-\mathrm{a} 7$ were selected with Hygromycin-B then compared against wild-type $\mathrm{CHO}(\mathrm{CHO}-\mathrm{WT})$ and non-transfected $\mathrm{CHO}-\mathrm{a} 7$ on the basis of their ability to bind and internalize fluorescent DiI-LDL cholesterol. Each CHO-a7 was treated overnight with Lovastatin, excess free sterols, or ethanol (a vehicle control) in lipoprotein-deficient serum. Lovastatin is an HMG-CoA reductase inhibitor that blocks the rate-limiting step in cholesterol synthesis ${ }^{424,440}$. By impeding the cells' endogenous 


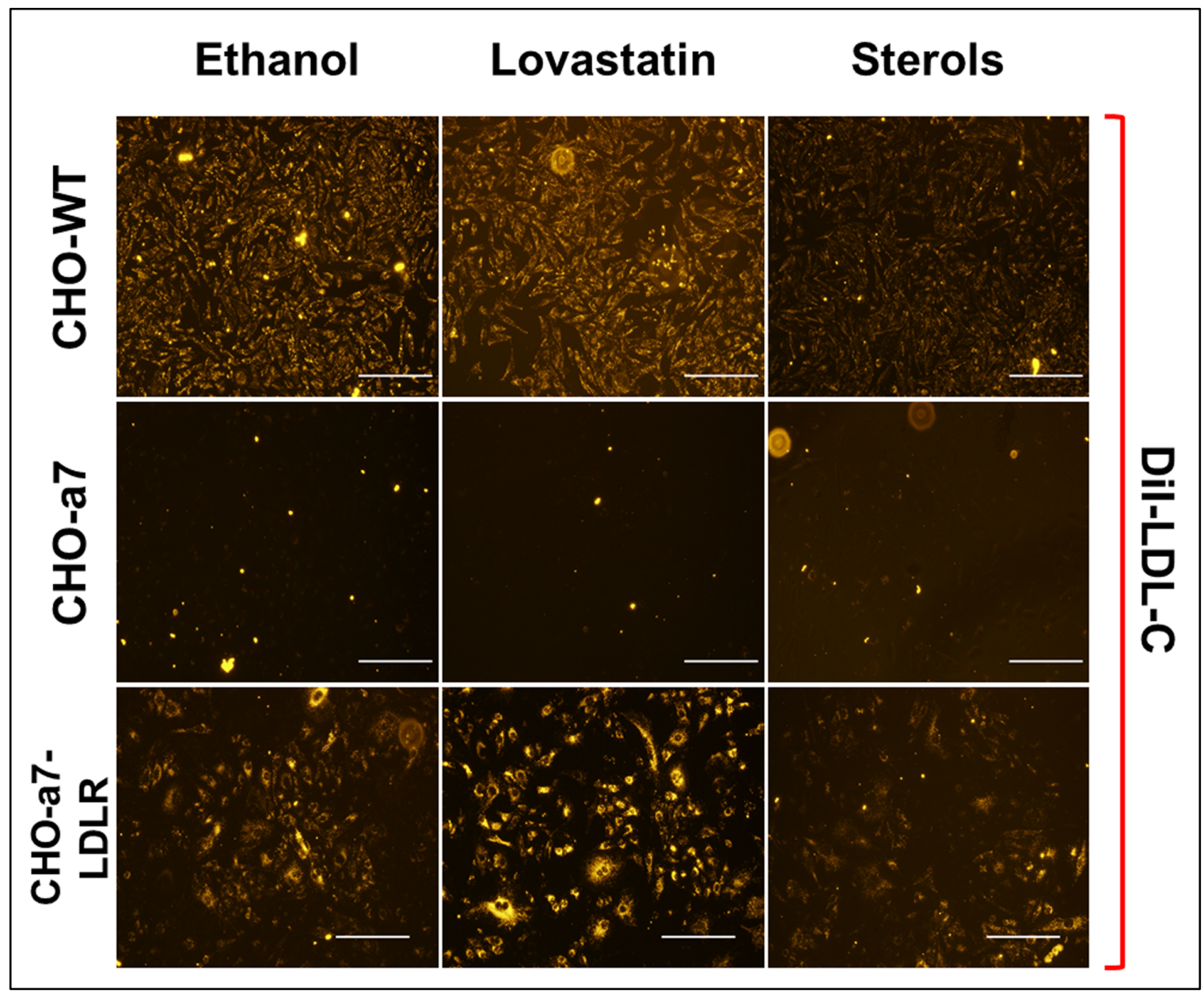

Figure 27. Transfected CHO-a7-LDLR exhibited LDL internalization sensitive to Lovastatin, excess sterols, or ethanol (vehicle control). This behavior paralleled that of CHO-WT under the same media conditions, and both were in stark contrast to the lack of LDL-C uptake seen in Ldlr-dysfunctional CHO-a7. 
production of LDL-C, Lovastatin facilitates exogenous cholesterol internalization via surface up-regulation of the LDLR, allowing the cell to still meet its metabolic requirements while having an overall therapeutic effect on serum cholesterol levels. Excess free sterols are membrane permeable. Consequently, they can be internalized by receptor-independent mechanisms that secondarily result in LDLR down-regulation ${ }^{441}$. Under such conditions, CHO-WT showed an expected pattern of maximum DiI-LDL internalization with Lovastatin treatment over the ethanol control, and almost complete abrogation of DiI-LDL uptake when pre-treated with excess free sterols. In contrast, CHO-a7 expectedly demonstrated virtually no DiI-LDL internalization regardless of the treatment condition. The transfected CHO-a7-LDLR exhibited DiI-LDL internalization that was appropriately sensitive to exogenous Lovastatin and demonstrated functional restoration of LDL receptor-mediated endocytosis. Exposure to excess sterols yielded a decrease in LDL-C internalization, while ethanol vehicle treatment yielded a slight increase. This behavior parallels that seen in $L d l r$-competent CHO-WT under the same experimental conditions, validating the $L D L R$ functionality and physiologic sensitivity of the episomal plasmid.

FH-iPSC transfection with $p E H Z-L D L R-L D L R$. FH-iPSC retain the same genetic dysfunction as their source patient fibroblasts and therefore offer limited therapeutic value with regards to restoring LDLR-mediated endocytosis. Since constitutive $L D L R$ transgene expression for treating FH has not yet achieved a cure ${ }^{442,443}$ and may even be cytotoxic ${ }^{444,445}$ we elected to use the plasmid $p E H Z-L D L R-L D L R$ to 
impart physiological responsiveness by $10 \mathrm{~kb}$ of up-stream genomic control sequences $^{424,446,447}$.

We initially confirmed $p E H Z-L D L R-L D L R$ physiological restoration of receptormediated endocytosis in the $L d l r$-deficient cell line CHO-a7 (Figure 27) ${ }^{424,439}$. Because of the plasmid's large $31 \mathrm{~kb}$ size, we utilized electroporation transfection ${ }^{448}$ and tested a wide range of parameters using both decaying exponential and square-wave electroporators (Table 4).

Using a BTX 630ECM decaying exponential electroporator with a $4 \mathrm{~mm}$ cuvette $^{448}$, we found that settings achieving time constants $(\tau)$ between 16 and $18 \mathrm{~ms}$ were optimal for achieving iPSC survival and transfection. Smaller $\tau$ were associated with increased iPSC survival but inadequate plasmid delivery, while $\tau$ greater than $18 \mathrm{~ms}$ resulted in cell death. We also tested the effects of temperature and buffer on electroporation success. For the conditions tested, pre-chilling all components was critical. Moreover, we found no difference with using either phosphate-buffered saline (PBS) or a commercially available electroporation buffer.

We then tested the NEPA21 square-wave electroporator using a range of parameters for plasmid delivery to human pluripotent stem cells. From the range of settings tested, a voltage of $300 \mathrm{~V}$ and pulse length time of $0.5 \mathrm{~ms}$ facilitated iPSC survival and successful delivery of the $31 \mathrm{~kb}$ plasmid. We then conducted all square-wave electroporations at room temperature in Opti-MEM media. 48 hours after electroporation, Hygromycin-B selection was initiated at $10 \mu \mathrm{g} / \mathrm{ml}$ for at least 5 days, followed by $0.5-1 \mu \mathrm{g} / \mathrm{ml}$ thereafter as a maintenance dose. 
pEHZ-LDLR-LDLR retention and functional restoration. $p E H Z-L D L R-L D L R$ is stably maintained as an episome and does not integrate into the host genome ${ }^{424}$. After culturing FH-iPSC-LDLR under continuous Hygromycin-B selection ${ }^{429}$ for over 6 months, we subjected the cells to alkaline lysis and recovered the plasmid via ethanol precipitation. The isolated plasmid was purified and digested with the restriction enzyme AgeI, yielding two appropriately sized fragments measuring $18.6 \mathrm{~kb}$ and $12.1 \mathrm{~kb}$ (Figure 28Ai). We then validated the functionality of the recovered plasmid by transfecting it into Ldlr-deficient CHO-a7 (CHO-a7-LDLR; Figure 28Aii). The recovered plasmid restored the physiological feedback control of LDLR-mediated endocytosis in the CHO-a7LDLR. These findings collectively demonstrate that (a) $p E H Z-L D L R-L D L R$ is retained as an episome in human iPSC and (b) its function is independent of genomic integration.

We next wanted to determine if $p E H Z-L D L R-L D L R$ had an effect on the inherent pluripotence of our FH-iPSC. Therefore, we assessed expression of TRA-1-60 on transfected cells cultured for over a year under Hygromycin-B antibiotic selection pressure. Like their non-transfected FH-iPSC counterparts (Figure 22C), FH-iPSCLDLR retained a robust expression of TRA-1-60 (Figure 28Bi). Expression of NANOG in $\mathrm{FH}-\mathrm{iPSC} \pm \mathrm{LDLR}$ was also confirmed by PCR (Figure 28Bii). These results suggest that transfection with the episomal plasmid $p E H Z-L D L R-L D L R$ does not diminish iPSC pluripotence.

To determine if FH-iPSC-LDLR derivatives also retained the plasmid and displayed functional restoration of LDLR-mediated endocytosis, we differentiated $\mathrm{FH}-\mathrm{iPSC} \pm \mathrm{LDLR}$ into FH-HLC \pm LDLR. The derived HLC were cultured with Lovastatin, excess free sterols, or ethanol vehicle control overnight in lipoprotein-deficient media prior to 


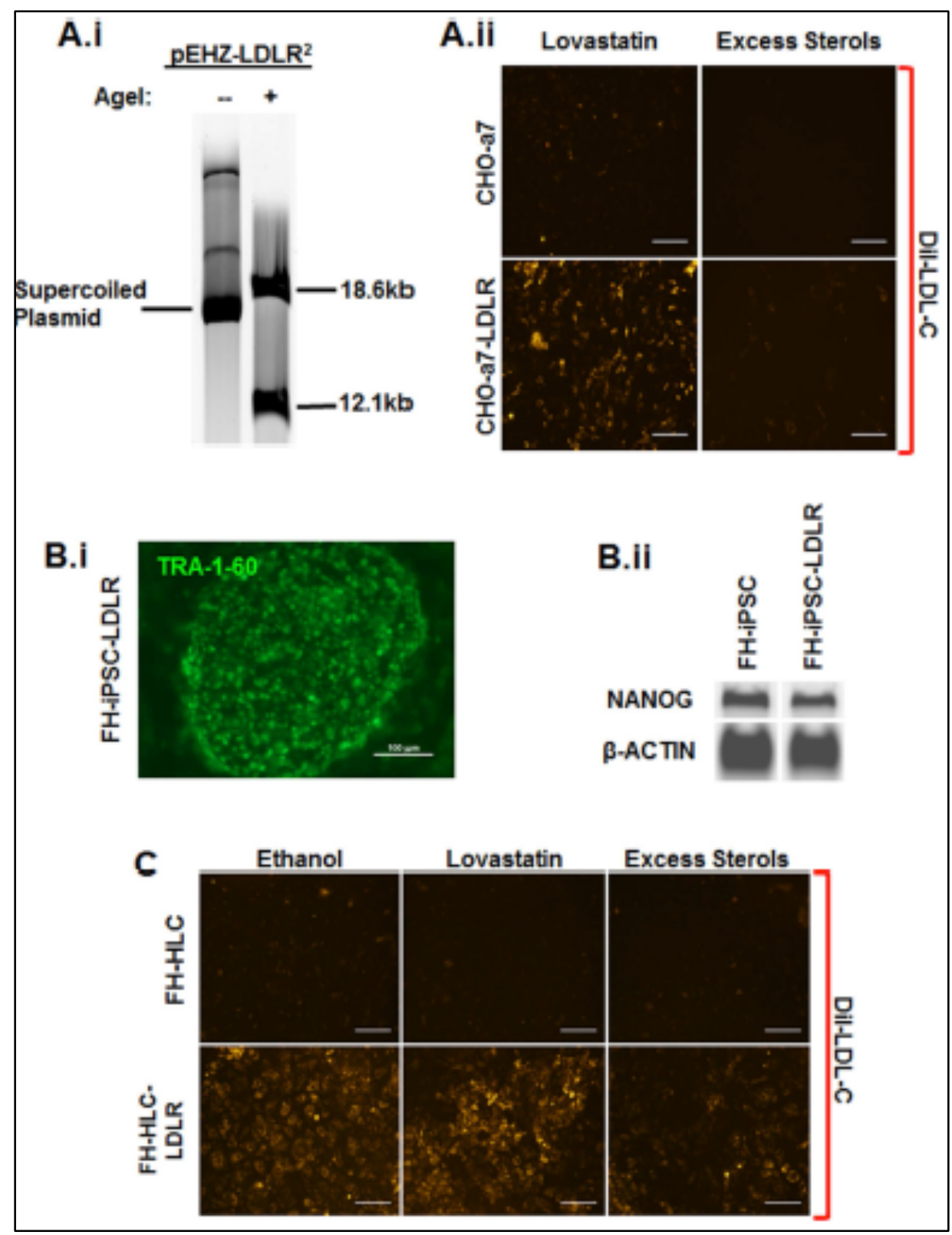

Figure 28. (A.i) $p E H Z-L D L R-L D L R$ episome was recovered from FH-iPSC-LDLR and cleaves into expected fragment sizes after digestion by AgeI-HF restriction enzyme. (A.ii) Recovered plasmid transfected into Ldlr-deficient CHO-a7 demonstrates restored physiologic receptor-mediated endocytosis via DiI-LDL internalization. (B.i) FH-iPSCLDLR colonies cultured for over 12 months still exhibit iPSC-characteristic well-defined borders and express TRA-1-60-488 via live-labeling (Scale bar $=100 \mu \mathrm{m})$. (B.ii) 
Pluripotence of long-term cultured FH-iPSC $\pm L D L R$ was verified by $P C R$, which demonstrated NANOG expression. $\beta$-actin was used as a loading control. (C) FH-HLCLDLR displayed differential physiologic sensitivity, with highest DiI-LDL internalization with exposure to Lovastatin and almost complete internalization abrogation with exposure to excess sterols. Little internalization of DiI-LDL was detected in FH-HLC. Ethanol was used as a vehicle control (Scale bar $=200 \mu \mathrm{m})$. 
incubation with DiI-LDL (Figure 28C). The FH-HLC demonstrated little internalization of DiI-LDL, indicating that without any transgene intervention, LDLR-mediated endocytosis of LDL remained dysfunctional. However, FH-HLC-LDLR exhibited markedly elevated LDL endocytosis that demonstrated the same physiological sensitivity to peripherally applied statin, sterols, or vehicle control as seen with the CHO-a7-LDLR (Figure 27). These findings indicate that $p E H Z-L D L R-L D L R$ effectively restores physiological feedback control of LDLR activity in FH-iPSC derivatives.

Restored receptor-mediated LDL endocytosis quantification. To facilitate the quantification of $p E H Z-L D L R-L D L R$-driven specific LDL internalization, we differentiated FH-iPSC \pm LDLR into mesenchymal cells (FH-MC \pm LDLR; Figure 29A) utilizing an established protocol from our lab ${ }^{425}$. These cells grow in a monolayer and are contact-inhibited, allowing quantification of specific LDL receptor-mediated endocytosis. FH-MC and FH-MC-LDLR were cultured in lipoprotein-deficient serum media supplemented with Lovastatin, excess sterols or ethanol control before exposure to DiILDL (Figure 29B). The ethanol control yielded appreciable, though not significant $(p=$ 0.27), differences in internalization between the two groups (FH-MC: $188.57 \pm 74.33$ vs. FH-MC-LDLR: $438.82 \pm 180.57$ fluorescence units/mg protein $(\mathrm{FU} / \mathrm{mg})$; mean $\pm \mathrm{SEM})$ reflecting the cellular lipid requirement due to the lipoprotein-deficient media environment. This difference was amplified when the cells were exposed to the additional stimulus of Lovastatin. Transgene-expressing cells displayed a statistically significant increase $(p=0.035)$ in specific internalization versus their non-transfected counterparts (FH-MC: $321 \pm 61.7$ FU/mg vs. FH-MC-LDLR: $526.15 \pm 21.18$ FU/mg). As expected, 


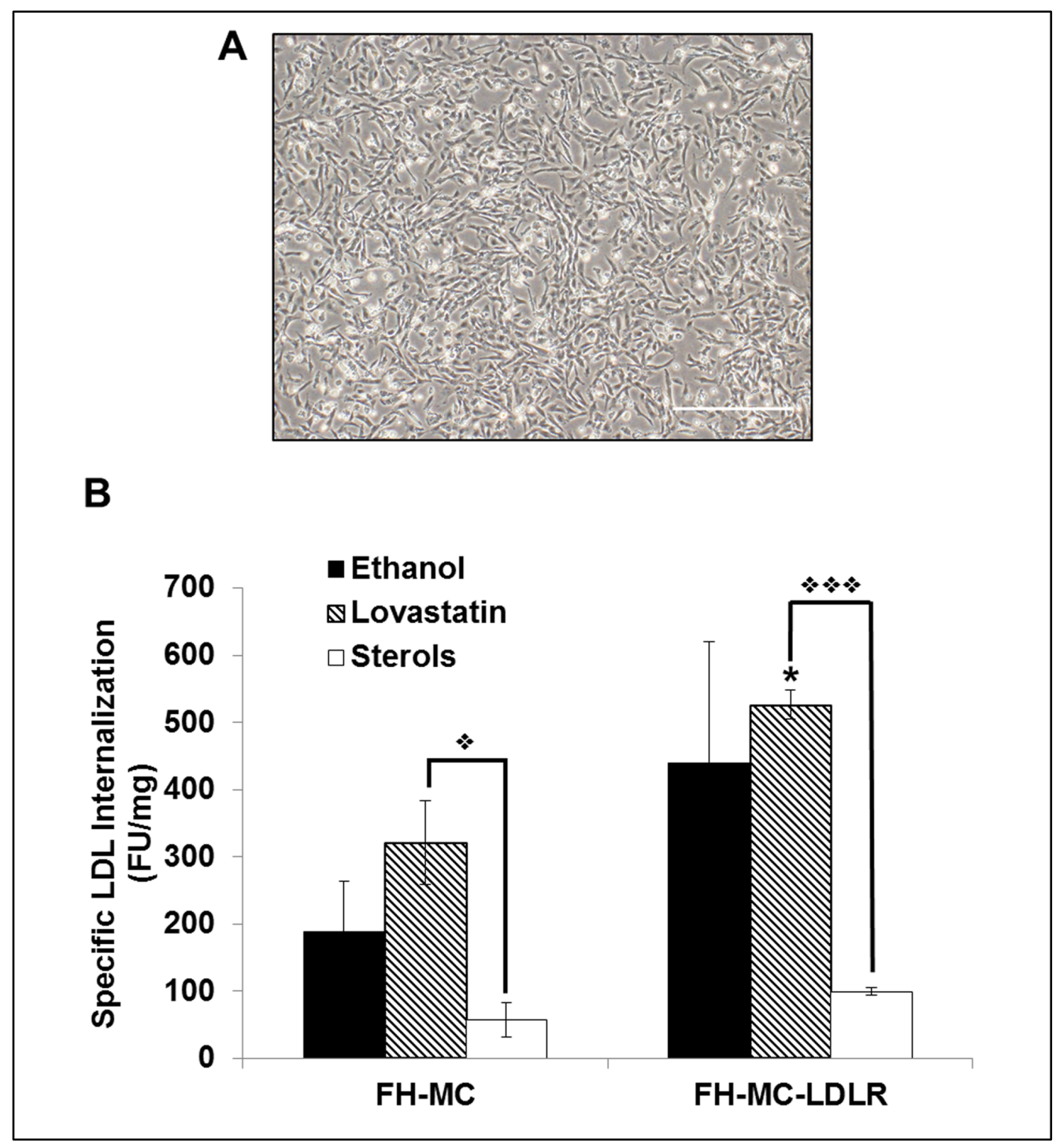

Figure 29. (A) Phase microscopy image of transfected FH-iPSC-derived mesenchymal cells $(F H-M C \pm L D L R)($ Scale bar $=500 \mu \mathrm{m})$ depicts a fibroblastic monolayer suitable for quantification studies. (B) Quantification of specific DiI-LDL internalization by FH-MC displayed impaired LDL internalization. Transfected FH-MC-LDLR demonstrated significantly greater ability to internalize DiI-LDL. Bars are depicted as means \pm S.E.M., and these graphs characterize experiments conducted in triplicate $\left(^{*},=p<0.05\right.$ and $* *=p<0.001 ; *=$ significance between cell types and similar treatments, while = significance within a cell type). 
free sterols suppressed DiI-LDL internalization to a statistically equivalent level regardless of transgene presence (FH-MC: $56.90 \pm 25.93$ vs. FH-MC-LDLR: $99.15 \pm$ $5.80 \mathrm{FU} / \mathrm{mg}, p=0.19)$ since free sterol internalization is receptor independent ${ }^{449}$. Within the transfected FH-MC-LDLR population, the suppression elicited by free sterols was extremely significant when compared to Lovastatin $(p=0.00004)$ and significant with regards to the ethanol control $(p=0.02)$. Presenting these differences another way, transfected FH-MC-LDLR demonstrated 1.63- and 2.33-fold increases in specific DiILDL internalization over FH-MC in the presence of Lovastatin and ethanol, respectively. These findings suggest that the $p E H Z-L D L R-L D L R$ plasmid functionally restores LDL receptor-mediated endocytosis without direct genetic manipulation in FH-iPSC-derived progeny.

\section{DISCUSSION}

Familial Hypercholesterolemia (FH) is a heritable disease of LDL receptor (LDLR) dysfunction. While the only known cure for FH is liver transplant, unfortunately, the availability of donor livers pales in comparison to a high demand. Furthermore, there are long-term issues to consider with transplant, such as compatibility, cost, quality-of-life, and possibly death. Because the availability of quality hepatocytes is a key issue, induced pluripotent stem cells (iPSC) presented us with a means of generating a sufficient quantity of cells that we could then differentiate into hepatocyte-like cells (HLC). In addition to developing a large quantity of cells, iPSC have the added benefit of being obtained in an autologous manner, potentially mitigating issues with rejection and obviating the need for immunosuppressive agents. However, a key hurdle with this 
approach is that if we were to use FH patient-derived iPSC (FH-iPSC), these cells and their derivatives would contain the same LDLR dysfunction as their parent cells, and would therefore provide little to no therapeutic benefit.

Thus, we developed a proof-of-concept strategy that carries broad implications for generating and functionally restoring genetically deficient iPSC. To do so, we utilized a transient reprogramming vector as well as non-viral, episomal vectors that confer genomic LDLR transgene control. Based on these methods, we made the following significant findings:

(a) An episomal, transgene plasmid can be maintained extra-chromosomally within iPSC.

(b) This plasmid does not impair the pluripotency of iPSC.

(c) Genomic control of plasmid LDLR transgene expression restores physiologic functionality to genetically deficient cells.

We used synthetic transient RNA (i.e., modRNA) to reprogram FH-fibroblasts into FH-iPSC. Our relatively low reprogramming efficiency with modRNA was considerably lower than published figures (approaching $2 \%{ }^{234}$ ). This efficiency could result from the LDLR genetic deficiency of our cells, though several other reports describing FH-iPSC generation with viral vectors fail to mention reprogramming efficiencies ${ }^{89,270,450}$. Therefore, our efficiency of $0.016 \%$ could potentially be attributed to experimental variation. Furthermore, as iPSC are highly proliferative, overall efficiency is not a significant concern since, theoretically, only one iPS cell is required to generate a whole colony of cells. 
Approaches to genetically correct the LDLR deficiency in FH via gene therapies have traditionally utilized viral vectors ${ }^{127,451-453}$. As an example, some have used the portal vein to dispense infectious viral particles directly to the liver. In animals, two distinct outcomes were observed:

(1) Circulating LDL cholesterol levels were significantly, but transiently, reduced ${ }^{451}$.

(2) LDL cholesterol levels did not reach targeted goals due to potential plasmid $\operatorname{loss}^{454}$, immune detection of transduced cells ${ }^{453}$, inactivation of the LDLR transgene $^{455}$, or a suboptimal transduction of host hepatocytes ${ }^{451,452}$.

Viral vectors such as adenoviruses or retroviruses have insert capacities typically less than $10 \mathrm{~kb}$. Because of this relatively small size, the options for controlling transgene expression are limited ${ }^{456}$. For instance, the use of Cytomegalovirus (CMV) or Rous Sarcoma Virus (RSV) promoters to control LDLR transgene expression led to excessive lipid internalization, causing cell death ${ }^{130,131}$. Lipid accumulation was observed in the livers of Watanabe heritable hyperlipidemia (WHHL) rabbits given a CMV-driven $L D L R$ in E1-deleted adenovirus ${ }^{457}$. To contrast, recent reports have described the use of hepatocyte-specific promoters, such as thyroid-binding globiulin or alpha-1 anti-trypsin (A1AT), to constitutively drive $L D L R$ transgene expression. In these instances, cytotoxic lipid accumulations were not seen ${ }^{127,451}$. Therefore, hepatocyte-specific promoters may ultimately protect against lipid accumulation, though their mechanism of action has not been specified ${ }^{458}$. However, given that functional LDL receptors are physiologically regulated via a feedback loop, the constitutive expression of $L D L R$ may have long-term, detrimental consequences. 
Alternatively, episomal plasmids containing genomic DNA expression control sequences can be used to address the $L D L R$ deficiency in FH. These genomic sequences can confer precise physiological control of the $L D L R$ transgene while enhancing longterm $L D L R$ expression ${ }^{459}$. We utilized the episomal plasmid $p E H Z-L D L R-L D L R$, which contains the EBV retention and replication sequences EBNA1 and oriP. These sequences allow $p E H Z-L D L R-L D L R$ to be maintained as an episome ${ }^{6}$. The EBNA1 trans regulatory protein, in particular, is unique for two reasons. First, it is the only EBV protein required for retained episomal function ${ }^{460}$. Second, unlike the other EBV proteins, EBNA1 is not immunogenic ${ }^{461}$. Thus, host immune response to the plasmid is mitigated.

The expression of the LDLR is tightly controlled by an endogenous feedback $\operatorname{loop}^{462}$. Cells regulate surface LDLR expression based on circulating LDL levels and membrane cholesterol content ${ }^{462}$. Prior studies demonstrate that $p E H Z-L D L R-L D L R$ reestablished receptor-mediated endocytosis in FH fibroblasts and persisted long-term in non-dividing hepatocytes in vivo ${ }^{6}$, although the transfection efficiency was unclear. In our application, $p E H Z-L D L R-L D L R$ restored physiologically sensitive receptor-mediated endocytosis in FH-iPSC. Additionally, this function was retained in various FH-iPSCderived progeny.

To our knowledge, this is the first report demonstrating the delivery and stable transfection of a relatively large $(38 \mathrm{~kb})$ episomal plasmid into human iPSC using electroporation. Though we could have transfected the source FH fibroblasts prior to reprogramming, the effect of a large $31-\mathrm{kb}$ plasmid on reprogramming is unknown, as is the viability of the plasmid in the long term. Transfection of FH-iPSC-derived cells was 
also an option. However, these cells would still require long-term antibiotic selection, which is problematic with hepatocytes considering that they culture poorly ${ }^{423}$.

Electroporation provided us with another alternative transfection modality. Because others successfully delivered plasmids to hPSC using electroporation ${ }^{463,464}$, we sought to do the same with FH-iPSC. Electroporation generally presents several challenges. There are several parameters reported in the literature, many of which depend on buffers utilized, temperature, cuvette size, and even the type of machine used. For instance, decayed-exponential electroporation has been shown to be effective for prokaryote transfection, while square-wave electroporation is typically used for transfection of mammalian cells ${ }^{465}$. We used both decayed-exponential and square-wave electroporation, and produced viable, transfected FH-iPSC (FH-iPSC-LDLR) that were resistant to Hygromycin B selection. Though we observed relatively low transfection efficiencies, this phenomenon may be attributable to the sensitivity of pluripotent stem cells, which are difficult to transfect ${ }^{465}$, the large size of the plasmid, and the physical and chemical stresses of electroporation ${ }^{459}$. Further, it has been shown that the EBV plasmid transfection event partly destabilizes mechanisms of replication in the days following transfection taking about two weeks to achieve stability ${ }^{466}$. Though we were unable to precisely quantify transfection efficiency, an advantage of utilizing pluripotent stem cells is that even with low efficiency, a potentially unlimited number of transfected cells can be generated. Furthermore, if a sufficient quantity of cells carrying the genomic DNAcontrolled transgene could be delivered, then host homeostatic control of LDL metabolism could be restored without the need for constitutive $L D L R$ expression. 
Another issue to address with our approach is plasmid retention, a common problem seen with using episomal viral vectors for direct gene therapy to the liver ${ }^{467}$. EBV-based plasmids replicate once per cell cycle ${ }^{460}$ and, without antibiotic selection, are lost at a rate of 2 to $5 \%$ per cell division ${ }^{446}$. Therefore, using iPSC as a therapeutic cell source may require continuous selection until a stable phenotype (such as hepatocytes) can be derived. To that end, two approaches could be used to achieve this goal: (a) differentiate FH-iPSC-LDLR in vitro with antibiotic selection until a minimally proliferative state is reached prior to implantation, or (b) generate a liver-tissue mimic with stromal and vascular support that facilitates selection and phenotype stabilization prior to implantation.

Secondly, it is important to recognize that our strategy represents a functional, and not a genetic, correction and does not impede expression of the endogenous defective LDLR. There is potential for dysfunctional endogenous LDLR to interfere with the function of the corrected exogenous gene product. As most FH patients do not know exactly what their genetic dysfunction is and since there are over 1200 reported LDLR mutations affecting function ${ }^{57}$, it will be necessary to determine if specific mutations or classes of mutations prevent phenotype recovery by $p E H Z-L D L R-L D L R$. Though clinically classified as "homozygote" (GM01355; the source patient exhibited a maximum LDL of $700 \mathrm{mg} / \mathrm{dl}$ and presented with xanthomas), the FH cells used here demonstrated some LDLR activity and responsiveness to Lovastatin, suggesting that the patient may actually be a compound heterozygote ${ }^{468,469}$. Transfected cells showed an approximate 2-fold increase in DiI-LDL internalization and no inhibitory effect from the defective endogenous LDLR was detected ${ }^{468}$. This 2 -fold increase in internalization is 
potentially significant for three reasons: first, it demonstrates that internalization is being effected by the exogenous gene product and not just stimulation of the endogenous $\operatorname{LDLR}^{468}$. Second, it coincides with the observation that LDL-C internalization is $L D L R$ allelic in a gene-dose dependent manner ${ }^{462}$ and third, it demonstrates physiological control as having an upper limit ${ }^{130}$. The internalization data normalized to the ethanol control suggests a pattern of normal feedback regulation ${ }^{462}$. Put another way, though the non-transfected cells have a reduced capacity for LDL-C internalization, they display an expected physiological responsiveness paralleled by the transfected cells. The long-term viability of a cell-based therapy for FH may hinge on preventing excessive LDL-C uptake by any one cell.

Though our approach specifically targets the LDLR dysfunction in FH, a strategy using episomes with genomic control of transgenes can be tailored to other biological conditions or clinical deficiency with potential for amelioration by iPSC intervention, further enhancing its clinical potential in tissue engineering applications. 


\section{CHAPTER VI}

\section{SUMMARY AND CONCLUSIONS}

Each hepatocyte is capable of carrying out over 500 metabolic processes, making the liver one of the key metabolic regulatory organs in the body ${ }^{145}$. Therefore, it is not surprising that liver dysfunction can be detrimental to various systemic processes, inducing cardiovascular disease to neurological diseases, to even multi-organ failure and death. In several pathologies, liver transplant remains the only curative option, though the availability of high-quality, compatible organs for transplant is impeded by a severely restricted supply. Recent research emphases now include tissue-engineered solutions that aim to assist or replace ailing liver organs.

In this project, we used the heritable disease Familial Hypercholesterolemia (FH) as a model for which we could develop a functional liver-tissue mimic device. Over the span of this dissertation project, we accomplished three goals. First, we were the first to develop a proof-of-concept liver tissue mimic device by combining HepG2 model hepatocyte cells with parenchymal support provided by adipose-derived stromal vascular fraction (SVF) cells. This liver-tissue mimic was able to engraft with the host vasculature and clear peripherally injected, circulating LDL cholesterol ${ }^{9}$.

Second, one of the chief problems with liver transplantation (in addition to the inadequate supply of quality hepatocytes), and transplantation in general, is donor-host 
compatibility. To this end, we tailored our liver-tissue mimic device to FH patients by (a) using patient cells to develop induced pluripotent stem cells (iPSC), potentially mitigating issues with parenchymal supply; (b) functionally correcting the LDL receptor (LDL-R) deficiency in patient-derived iPSC, allowing patient-derived cells to be restored in their LDL-R functionality; (c) differentiating corrected iPSC into hepatocyte-like cells (HLC), as hepatocytes traditionally express the highest levels of LDL-R in the body; and (d) promoting the engraftment of HLC in vivo by using SVF, since hepatocytes require vascular support for survival and function. Most importantly, functionalized HLC were able to internalize peripheral LDL, unlike their genetically deficient counterparts, which retain the LDL-R dysfunction. Here, we were the first to use entirely non-viral, nonintegrating modalities for generating and functionally correcting these cells, potentially mitigating adverse future outcomes associated with viral-vector usage ${ }^{1}$. Moreover, with further development, functionalized HLC could serve as a bridge-to-transplantation or mitigate transplantation entirely, since FH patients exhibit otherwise entirely normal liver function.

Third, the vasculature is essential to liver-tissue mimic functionality. In our proofof-concept liver-tissue mimic, we observed that 3D constructs with HepG2 model hepatocytes lacking SVF were unable to internalize circulating LDL cholesterol. In our FH-specific iPSC-derived liver-tissue mimic, 3D HLC constructs lacking SVF support expressed significantly less albumin, suggesting that the HLC were either less functional (i.e., albumin output was reduced) or did not survive. Thus, both of these findings suggest that SVF is essential for parenchymal cell functionality, phenotype maintenance, and likely even survival. Thus, we wished to examine the means by which SVF 
spontaneously assembled into a functional vasculature, allowing it to critically support metabolically active cells such as hepatocytes. Here, we were the first to show that the Wnt signaling pathway is broadly implicated in hSVF endothelial cell (EC) vascular networking, and that the Wnt5a isoform in particular acts to modulate the assembly, not complexity, of hSVF vascular networks.

These efforts have resulted in a methodology that can be used to create organtissue autologous, iPSC-derived parenchyma supported by adipose-derived SVF. We also have the means to correct inherent genetic deficiency to restore functionality to these cells, such that these organ-tissue mimics impart a therapeutic value. Because SVF is critical to the ultimate functionality of these constructs (i.e., to produce a lasting, functional tissue-engineered device), our discovery of one regulatory mechanism in SVF vascular assembly provides us with a means of modulating the vasculature to meet different needs. Obviously, other mechanistic components are of interest, and will be discussed in greater detail later on.

With respect to future efforts, it is academically reassuring to see that our findings raise more questions than they have answered. Several issues to examine revolve around our generation of a liver-tissue mimic. One topic of interest is the intricate, intimate relationship between normal liver hepatocytes and the sinusoidal vasculature within the liver. The liver vasculature not only promotes hepatocyte survival, but also plays a critical role in the exchange between systemic circulation and hepatocytes. Normal hepatocytes modulate their microenvironment to promote liver sinusoidal endothelial cell network formation, facilitating vital bulk transport. Our findings suggest that implanted hepatocytes also influence their microenvironment as demonstrated by their intimate 
interaction with adipose-derived SVF - an interaction that was seen in $3 \mathrm{D}$ constructs using either HepG2 or iPSC-derived HLC. Thus, one logical question is how do hepatocytes recruit an adipose SVF-derived vasculature? To this end, we could investigate the patterns in vascular assembly of SVF in isolation compared to SVF in direct or indirect contact with hepatocytes. Several assays (such as PCR, western blot, ELISA, as well as proteomics to name a few) would allow us to identify the proteins that mediate this interaction.

From this, a second topic to investigate would be the adaptation of the vasculature towards a hepatic phenotype. As mentioned in the background, embryologic development of the liver is actually made possible through intimate interactions with the cardiac mesoderm as well as the septum transversum mesenchyme. In this process, angioblasts (precursors to the liver sinusoids) are interspersed amongst cords of hepatoblasts (the precursors to hepatocytes and cholangiocytes). Thus, these two precursors are in close interaction throughout the liver's development. Since HLC are regarded to be immature (they express a significant proportion of AFP even by the end of directed differentiation), their combination with SVF may ultimately form a vasculature that represents a sinusoidal, fenestrated system as opposed to a vascular system comprised of tight junctions and a continuous basement membrane. Here, we could look for liver sinusoidal endothelial cell (LSEC) markers (such as LYVE-1 or SE-1, for example) as well as use various microscopy techniques to identify a sinusoidal ultrastructure.

A third issue lies with the development of the HLC themselves. In this regard, we have just stated that the HLC are, to a degree, immature. The presence of AFP and Albumin (in terms of gene and protein expression) suggests that our directed 
differentiation protocols do not yield a population of cells that are uniform in their maturity. Thus, one question that stems from this is: do HLC need to be mature to impart a functional, therapeutic effect? Related to this, in the context of $\mathrm{FH}$, it would be interesting to see if immature and mature HLC express LDL-R to varying degrees, or experience differences in LDL-R-mediated uptake and metabolism of LDL cholesterol. To this end, we have shown that HLC can perform this task, but have yet to demonstrate differences with respect to functional primary hepatocytes. Furthermore, if mature cells were desired over immature cells, would having HLC interspersed with SVF cells in 2D or $3 \mathrm{D}$ environments accelerate their maturity?

With regards to the vasculature, an issue requiring further examination is Wnt5a's role in SVF vascular assembly. For instance, with Wnt5a, we are still unaware of precisely which cells endogenously produce this ligand. Our data indicates that Wnt5a is common in hSVF cultures. As previously discussed, hSVF represents a heterogeneous cell population that includes endothelial cells, smooth muscle cells, fibroblasts, macrophages, pericytes, and various types of stem cells. Others have suggested that Wnt5a from macrophages, pericytes, or endothelial cells plays a role in modulating angiogenesis, but many of these efforts combine pure endothelial populations with transformed or immortalized stromal support cells. Our findings provide a basis for further investigation in heterogeneous populations such as SVF, but to date, the mechanisms by which SVF self-assembles still remains to be fully elucidated. Though our initial experiments with SVF show varying proportions of endothelial, perivascular, and stem cell populations between fresh and cultured SVF isolates, we could isolate EC alone using flow cytometry or MACS and see if these cells form networks of similar 
complexity as those seen in full, heterogeneous populations. In a reductionist approach, we could then supplement FACS- or MACS-isolated SVF subpopulations to attempt to restore the extent of vascular self-assembly seen with the complete isolation. In all of these experiments, Wnt5a expression at the gene and protein level could be assessed to determine (a) which cells express the genes for Wnt5a production and/or (b) if Wnt5a production requires two or more cells to communicate with one another. From (b), the modality of communication (juxtacrine or paracrine) could also be determined.

Our work also suggests a potential signaling axis via the prominent expression of the Wnt5a receptor Frizzled-4 (Fzd4). However, we do not yet know if the binding of Wnt5a to Fzd4 is precisely what drives changes in SVF vascular complexity. One simple experiment to establish the role of Fzd4 in this phenomenon would be to use a Fzd4 blocking peptide or to knockdown Fzd4 expression via RNA interference. We could then determine if the resulting vascular phenotype is comparable to that seen when Wnt5a neutralizing antibody was utilized. If the resulting vasculature were the same phenotype as untreated cultures, this would indicate that Fzd4 signaling may not play a role in vascular patterning. If a completely different vascular assembly outcome were seen, different from the outcomes seen with untreated and Anti-Wnt5a-treated cultures, this finding would provide us with yet another mechanistic axis to pursue later on. Further, in this scenario, it is possible that this axis may involve other Wnt isoforms, as Fzd4 can also bind $\mathrm{Wnt}^{470}$ as well as Norrin (a protein associated with retinal vascular development $)^{471}$. Since our experimental data showed that FZD4 was expressed in hSVF cultures, one hypothesis could be that Wnt5a binding of Fzd4 initiates a signaling cascade that leads to the modulation of hSVF vascular self-assembly. To this end, we could 
examine the expression of downstream markers from Wnt5a-Fzd4 binding. For example, Umbhauer and colleagues demonstrated that Wnt5a-Fzd4 signaling activates $\beta$-catenin ${ }^{472}$. Another downstream product of Fzd4 signaling (not yet shown with Wnt5a as the ligand) is Protein Kinase $\mathrm{C}^{473}$. These downstream markers could be used to verify upstream activity, such as the addition or removal of the Wnt5a ligand or receptor.

Lastly, our results indicate that adding rhWnt5a to cultures devoid of other Wnt isoforms, stimulates an increase in EC sheeting. This level of sheeting was absent in cultures not treated with the Wnt release inhibitor IWP2. One possibility here is that other Wnt isoforms work with Wnt5a to promote hierarchy, branching, and overall organization and complexity. Wnt5a is but one mediator. Furthermore, the blockage of Wnt5a via a neutralizing antibody does not completely abrogate network formation, though this antibody may not completely block the activity of Wnt5a. Blockade of all Wnt isoforms via IWP2 produced a greater decrease in vascular network formation as opposed to blockade of just Wnt5a, though Wnt5a does play a significant role in this process. These observations further suggest that Wnt5a modulates vascular networking (Figure 30) along with other Wnt isoforms, or that Wnt5a affects the vascular complexity but other (non-Wnt) factors (such as VEGF, PDGF-B, etc.) mediate vascular selfassembly. Future research efforts will likely uncover a constellation of Wnt isoforms and related molecules (such as Norrin, as an example) that participate in SVF self-assembly.

Broadly speaking, we have established the methods for a complex solution. In addition to demonstrating that SVF-derived vasculatures support the engraftment and functionality of implanted hepatocytes, we provide a platform for the engraftment of other parenchymal cell types. In addition to showing that Wnt5a mediates SVF vascular 


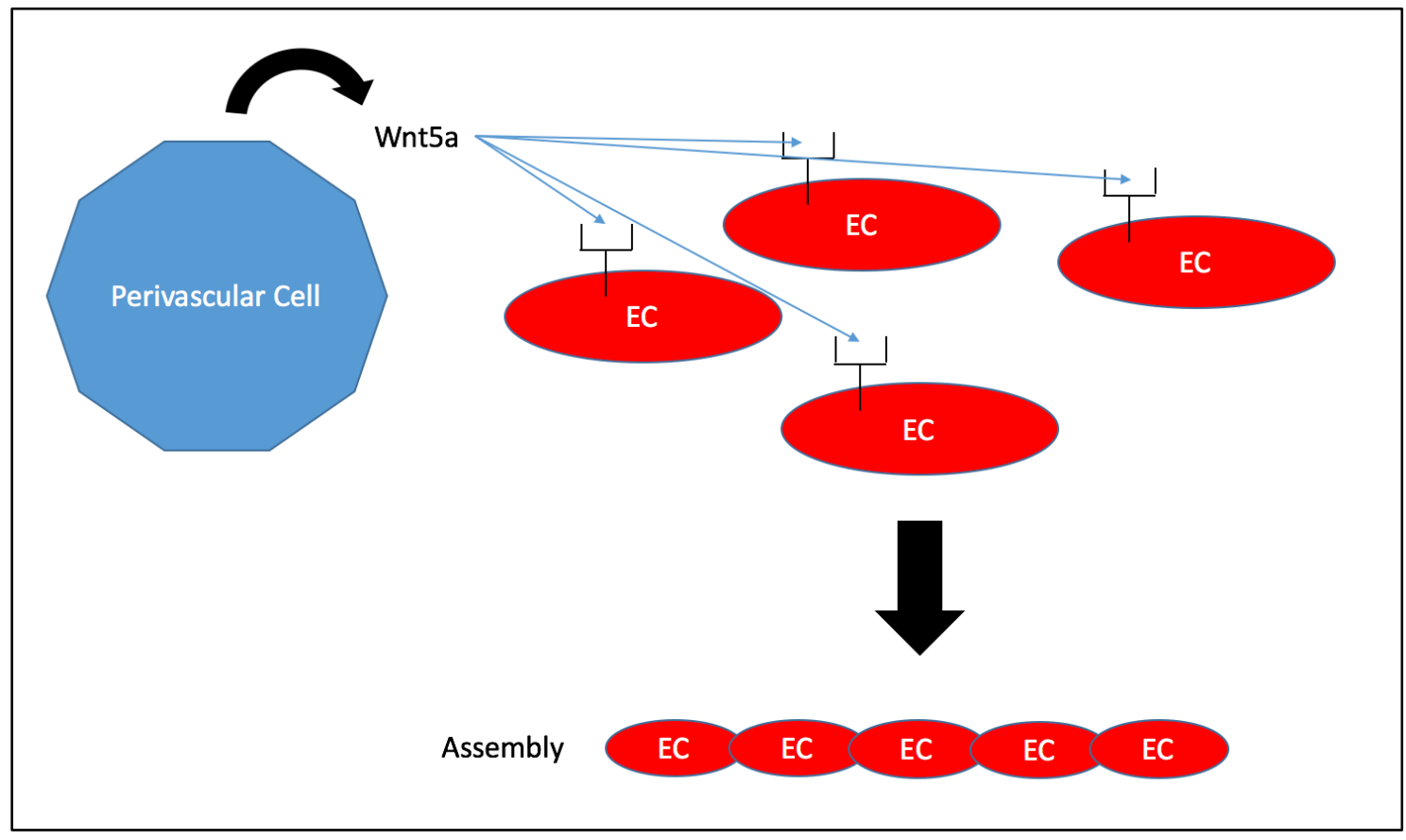

Figure 30. We have demonstrated that Wnt5a modulates hSVF EC self-assembly into vascular networks. However, there are several unknowns: (1) Which cells release Wnt5a? (2) Which receptor(s) is required? (3) Where is this receptor located? (We hypothesize that the Wnt5a receptor that has a role in mediating self-assembly is found on endothelial cells, as shown above). (4) Does Wnt5a modulate extravascular activity, such as connective tissue phenotype? These are just a few examples of future research avenues to consider. 
self-assembly, we also suggest that Wnt5a can be a target for manipulating derived vasculatures. And with respect to our final aim, in addition to generating and functionally restoring the LDL-R activity of autologous FH-iPSC, we provide a general platform for the use of non-viral, non-integrating modalities. Our efforts also provide a platform to generate tissue mimics for a variety of therapies. SVF cells could be utilized for vascular support, while iPSC-derived parenchyma could be generated and genetically manipulated. Lastly, we provide a strategy for gene therapy for any derived cell type, since iPSC can theoretically generate all cell types and can be obtained autologously. In sum, the data presented in this dissertation provides us with the necessary components for the generation and testing of a functional, therapeutic, autologous liver-tissue mimic.

In several of these cases, we would be afforded the opportunity not only to study the functionalization of various components of a liver-tissue mimic, but also the mechanisms behind how these components interact with one another. Such a mimic would potentially allow us to better understand how native liver processes take place without having to rely on primary cell isolation. This would also facilitate the development and screening of various therapeutic agents. Not only would these investigations provide us with further insight into how a tissue-engineered liver-tissue mimic might function in a therapeutic, translational capacity, but it would lay the groundwork for the rapid development and modulation of other organ-tissue mimics as well. This could have a profound impact on traditionally challenged medical fields such as transplantation. 


\section{REFERENCES}

1 Ramakrishnan, V. M. et al. Restoration of Physiologically Responsive LowDensity Lipoprotein Receptor-Mediated Endocytosis in Genetically Deficient Induced Pluripotent Stem Cells. Sci Rep 5, 13231, doi:10.1038/srep13231 (2015).

2 Al-Allaf, F. A. et al. LDLR-Gene therapy for familial hypercholesterolaemia: problems, progress, and perspectives. Int Arch Med 3, 36, doi:10.1186/17557682-3-36 (2010).

3 Grossman, M. R., SE; Kozarsky, K; Stein, EA; Engelhardt, JF; Muller, D; Lupien, PJ; Wilson, JM. Successful ex vivo gene therapy directed to liver in a patient with familial hypercholesterolemia. Nature genetics 6, 335-341 (1994).

4 Yamanaka, S. A fresh look at iPS cells. Cell 137, 13-17, doi:10.1016/j.cell.2009.03.034 (2009).

$5 \quad$ SoRelle, R. Human Gene Therapy: Science Under Fire. Circulation 101, e90239024 (2000).

6 Hibbitt, O. C. et al. Long-term physiologically regulated expression of the lowdensity lipoprotein receptor in vivo using genomic DNA mini-gene constructs. Mol Ther 18, 317-326, doi:10.1038/mt.2009.249 (2010). 
7 Tabas, I. Consequences of cellular cholesterol accumulation: basic concepts and physiological implications. Journal of Clinical Investigation 110, 905-911, doi:10.1172/jci200216452 (2002).

8 Chen, A. A. et al. Humanized mice with ectopic artificial liver tissues. Proc Natl Acad Sci USA 108, 11842-11847, doi:10.1073/pnas.1101791108//DCSupplemental (2011).

9 Nunes, S. S. et al. Generation of a functional liver tissue mimic using adipose stromal vascular fraction cell-derived vasculatures. Sci Rep 3, 2141, doi:10.1038/srep02141 (2013).

10 Berg, J. M., Tymoczko, J. L., Gatto Jr., G. J. \& Stryer, L. Biochemistry 8th Edition. (Freeman \& Company, W. H., 2015).

11 Trapani, L., Segatto, M. \& Pallottini, V. Regulation and deregulation of cholesterol homeostasis: The liver as a metabolic "power station". World $J$ Hepatol 4, 184-190, doi:10.4254/wjh.v4.i6.184 (2012).

12 Lehninger, A. L., Nelson, D. L. \& Cox, M. M. Lipid Biosynthesis. (Freeman, W. H., 2010).

13 Liscum, L. in Biochemistry of Lipids, Lipoproteins, and Membranes, 5th Ed. (eds D. E. Vance \& J. E. Vance) Ch. 14, 399-421 (Elsevier, 2002).

14 Gropper, S. S. \& Smith, J. L. Advanced nutrition and human metabolism, 6th ed., (Wadsworth/Cengage Learning, 2013).

15 Lee, T. H. By the way, doctor. I just got my cholesterol checked, and I'm confused. LDL is bad and HDL is good, so how can they be combined into an overall number that makes any sense? Isn't that like an accountant mixing debits 
with credits? Wouldn't it make more sense to have a net cholesterol number (HDL minus LDL), or perhaps a ratio? Harv Health Lett 28, 8 (2003).

16 Goldstein, J. L. \& Brown, M. S. A century of cholesterol and coronaries: from plaques to genes to statins. Cell 161, 161-172, doi:10.1016/j.cell.2015.01.036 (2015).

17 El Harchaoui, K. E. et al. High-Density Lipoprotein Particle Size and Concentration and Coronary Risk. Ann Intern Med 150, 84-93 (2009).

18 Rifai, N., Warnick, G. R. \& Dominiczak, M. H. Handbook of Lipoprotein Testing. (Amer. Assoc. for Clinical Chemistry, 2000).

19 Asztalos, B. F., Tani, M. \& Ishida, B. in The HDL Handbook: Biological Functions and Clinical Implications (ed T. Komoda) Ch. 3, 37-64 (Elsevier, 2014).

20 Takata, K., Horiuchi, S., Rahim, A. T. M. A. \& Morino, Y. Receptor-mediated internalization of high-density lipoprotein by rat sinusoidal liver cells: identification of a nonlysosomal endocytic pathways by fluorescence-labeled ligand. J Lipid Res 29, 1117-1126 (1988).

21 Anderson, R. G. W., Brown, M. S. \& Goldstein, J. L. Role of the Coated Endocytic Vesicle in the Uptake of Receptor-Bound Low Density Lipoprotein in Human Fibroblasts. Cell 10, 351-364 (1977).

22 Schoenheimer, R. \& Breusch, F. Synthesis and destruction of cholesterol in the organism. J Biol Chem 103, 439-448 (1933).

23 Brown, M. S. \& Goldstein, J. L. Regulation of the Activity of the Low Density Lipoprotein Receptor in Human Fibroblasts. Cell 6, 307-316 (1975). 
24 Brown, M. S. \& Goldstein, J. L. A receptor-mediated pathway for cholesterol homeostasis. Science 232, 34-47 (1986).

25 Yokoyama, C. et al. SREBP-1, a basic-helix-loop-helix-leucine zipper protein that controls transcription of the low density lipoprotein receptor gene. Cell $\mathbf{7 5}$, 187-197 (1993).

26 Hua, X., Nohturfft, A., Goldstein, J. L. \& Brown, M. S. Sterol resistance in CHO cells traced to point mutation in SREBP cleavage-activating protein. Cell 87, 415426 (1996).

27 Sakai, J., Nohturfft, A., Goldstein, J. L. \& Brown, M. S. Cleavage of sterol regulatory element-binding proteins (SREBPs) at site-1 requires interaction with SREBP cleavage-activating protein. Evidence from in vivo competition studies. $J$ Biol Chem 273, 5785-5793 (1998).

28 Sakai, J. et al. Molecular identification of the sterol-regulated luminal protease that cleaves SREBPs and controls lipid composition of animal cells. Molecular Cell 2, 505-514, doi:Doi 10.1016/S1097-2765(00)80150-1 (1998).

29 Radhakrishnan, A., Sun, L. P., Kwon, H. J., Brown, M. S. \& Goldstein, J. L. Direct binding of cholesterol to the purified membrane region of SCAP: mechanism for a sterol-sensing domain. Mol Cell 15, 259-268 (2004).

30 Motamed, M. et al. Identification of luminal Loop 1 of Scap protein as the sterol sensor that maintains cholesterol homeostasis. J Biol Chem 286, 18002-18012, doi:10.1074/jbc.M111.238311 (2011).

31 Sun, L. P., Seemann, J., Goldstein, J. L. \& Brown, M. S. Sterol-regulated transport of SREBPs from endoplasmic reticulum to Golgi: Insig renders sorting 
signal in Scap inaccessible to COPII proteins. Proc Natl Acad Sci U S A 104, 6519-6526 (2007).

32 Rawson, R. B. et al. Complementation cloning of S2P, a gene encoding a putative metalloprotease required for intramembrane cleavage of SREBPs. Mol Cell 1, 4757 (1997).

33 Shimano, H. et al. Overproduction of cholesterol and fatty acids causes massive liver enlargement in transgenic mice expressing truncated SREBP-1a. J Clin Invest 98, 1575-1584 (1996).

34 Horton, J. D. et al. Combined analysis of oligonucleotide microarray data from transgenic and knockout mice identifies direct SREBP target genes. Proc Natl Acad Sci U S A 100, 12027-12032 (2003).

35 Brown, A. J., Sun, L. P., Feramisco, J. D., Brown, M. S. \& Goldstein, J. L. Cholesterol addition to ER membranes alters conformation of SCAP, the SREBP escort protein that regulates cholesterol metabolism. Mol Cell 10, 237-245 (2002).

36 Yang, T. et al. Crucial step in cholesterol homeostasis: sterols promote binding of SCAP to INSIG-1, a membrane protein that facilitates retention of SREBPs in ER. Cell 110, 489-500 (2002).

37 Ignatowski, A. I. Ueber die Wirkung der tierschen Einweisse auf der Aorta. Virchow's Arch Pathol Anat 198, 248 (1909).

38 Anitschkow, N. \& Chalatow, S. Ueber experimentelle Cholester-insteatose und ihre Bedeutung fuer die Entstehung einiger pathologischer Prozesse. Zentrbl Allg Pathol Pathol Anat 24, 1-9 (1913). 
39 Keys, A. Seven Countries. A Multivariate Analysis of Death and Coronary Heart Disease. (Harvard Univ. Press., 1980).

40 Gofman, J. W. et al. Blood Lipids and Human Atherosclerosis. Circulation 2, $161-178(1950)$.

41 Dobbin, V., Gofman, J. W. \& Nichols, A. V. Influence of dietary factors upon human serum lipoprotein concentrations. . Geriatrics 12, 7-17 (1957).

42 Study, F. H. Research Milestones, $<$ https://www.framinghamheartstudy.org/about-fhs/research-milestones.php $>$ (2015).

43 Mackness, M. I., Arrol, S. \& Durrington, P. N. Paraoxonase prevents accumulation of lipoperoxides in low-density lipoprotein. FEBS Lett 286, 152154 (1991).

44 Hessler, J. R., Robertson, A. L., Jr. \& Chisolm, G. M., 3rd. LDL-induced cytotoxicity and its inhibition by HDL in human vascular smooth muscle and endothelial cells in culture. Atherosclerosis 32, 213-229 (1979).

45 Parthasarathy, S., Barnett, J. \& Fong, L. G. High-density lipoprotein inhibits the oxidative modification of low-density lipoprotein. Biochim Biophys Acta 1044, $275-283$ (1990).

46 Barter, P. et al. HDL cholesterol, very low levels of LDL cholesterol, and cardiovascular events. New England Journal of Medicine 357, 1301-1310, doi:DOI 10.1056/NEJMoa064278 (2007). 
47 Voight, B. F. et al. Plasma HDL cholesterol and risk of myocardial infarction: a mendelian randomisation study. The Lancet 380, 572-580, doi:10.1016/s01406736(12)60312-2 (2012).

48 Gotto, A. M., Jr. \& Brinton, E. A. Assessing low levels of high-density lipoprotein cholesterol as a risk factor in coronary heart disease: a working group report and update. J Am Coll Cardiol 43, 717-724, doi:10.1016/j.jacc.2003.08.061 (2004).

49 Berliner, J. A. et al. Atherosclerosis: basic mechanisms. Oxidation, inflammation, and genetics. Circulation 91, 2488-2496 (1995).

50 Libby, P., Ridker, P. M. \& Hansson, G. K. Progress and challenges in translating the biology of atherosclerosis. Nature 473, 317-325, doi:10.1038/nature10146 (2011).

51 Tabas, I., Williams, K. J. \& Boren, J. Subendothelial lipoprotein retention as the initiating process in atherosclerosis: update and therapeutic implications. Circulation 116, 1832-1844, doi:10.1161/CIRCULATIONAHA.106.676890 (2007).

52 Goldstein, J. B., MS. Binding and Degradation of Low Density Lipoproteins by Cultured Human Fibroblasts. JBC 249, 5153-5162 (1974).

53 Brown, M. S., Dana, S. E. \& Goldstein, J. L. Receptor-dependent hydrolysis of cholesteryl esters contained in plasma low density lipoprotein. Proc Natl Acad Sci US A 72, 2925-2929 (1975). 
54 Goldstein, J. L., Basu, S. K., Brunschede, G. Y. \& Brown, M. S. Release of low density lipoprotein from its cell surface receptor by sulfated glycosaminoglycans. Cell 7, 85-95 (1976).

55 Müller, C. Xanthomata, hypercholestero-lemia, angina pectoris. Acta Med Scand 89, $75-84$ (1938).

56 Khachadurian, A. K. THE INHERITANCE OF ESSENTIAL FAMILIAL HYPERCHOLESTEROLEMIA. Am J Med 37, 402-407 (1964).

57 Nordestgaard, B. G. et al. Familial hypercholesterolaemia is underdiagnosed and undertreated in the general population: guidance for clinicians to prevent coronary heart disease: consensus statement of the European Atherosclerosis Society. Eur Heart J 34, 3478-3490a, doi:10.1093/eurheartj/eht273 (2013).

58 Goldstein, J. L., Hobbs, H. H. \& Brown, M. S. in The Metabolic and Molecular Bases of Inherited Disease, 8th Ed. (eds C. R. Scriver, A. L. Beaudet, W. S. Sly, \& D. Valle) 2863-2914 (McGraw-Hill, Inc., 2001).

59 Nabel, E. G. Cardiovascular disease. New Engl J Med 349, 60-72 (2003).

60 Usifo, E. et al. Low-density lipoprotein receptor gene familial hypercholesterolemia variant database: update and pathological assessment. Ann Hum Genet 76, 387-401, doi:10.1111/j.1469-1809.2012.00724.x (2012).

61 Defesche, J. C. Low-Density Lipoprotein Receptor - Its Structure, Function, and Mutations. Seminars in Vascular Medicine 4, 5-11 (2004).

62 Austin, M. A., Hutter, C. M., Zimmern, R. L. \& Humphries, S. E. Familial hypercholesterolemia and coronary heart disease: a HuGE association review. Am J Epidemiol 160, 421-429 (2004). 
63 Horton, J. D., Cohen, J. C. \& Hobbs, H. H. PCSK9: a convertase that coordinates LDL catabolism. J Lipid Res Suppl, S172-177 (2009).

64 Cohen, J. C., Boerwinkle, E., Mosley, T. H. \& Hobbs, H. H. Sequence variations in PCSK9, low LDL, and protection against coronary heart disease. New Engl J Med 354, 1264-1272 (2006).

65 Brown, M. S., Faust, J. R., Goldstein, J. L., Kaneko, I. \& Endo, A. Induction of 3hydroxy-3-methylglutaryl coenzyme A reductase activity in human fibroblasts incubated with compactin (ML-236B), a competitive inhibitor of the reductase. $J$ Biol Chem 253, 1121-1128 (1978).

66 Informatics, I. I. f. H. (2011).

67 Kovanen, P. T., Bilheimer, D. W., Goldstein, J. L., Jaramillo, J. J. \& Brown, M. S. Regulatory role for hepatic low density lipoprotein receptors in vivo in the dog. Proc Natl Acad Sci U S A 78, 1194-1198 (1981).

68 Vega, G. L. \& Grundy, S. M. Influence of lovastatin therapy on metabolism of low density lipoproteins in mixed hyperlipidaemia. J Intern Med 230, 341-350 (1991).

69 Mabuchi, H. et al. Reduction of serum cholesterol in heterozygous patients with familial hypercholesterolemia. Additive effects of compactin and cholestyramine. New Engl J Med 308, 609-613 (1983).

70 Uauy, R., Vega, G. L., Grundy, S. M. \& Bilheimer, D. M. Lovastatin therapy in receptor-negative homozygous familial hypercholesterolemia: lack of effect on low-density lipoprotein concentrations or turnover. J Pediatr 113, 387-392 (1988). 
71 Stein, E. A. Management of dyslipidemia in the high-risk patient. Am Heart J 144, S43-50 (2002).

72 Robinson, J. G. et al. Efficacy and safety of alirocumab in reducing lipids and cardiovascular events. $\quad N$ Engl J Med 372, 1489-1499, doi:10.1056/NEJMoa1501031 (2015).

73 Marenah, C. in Clinical biochemistry: metabolic and clinical aspects Vol. 35 (eds W. Marshall \& S. Bangert) 621-640 (1995).

74 Burneis, K. et al. Ezetimibe alone or in combination with simvastatin increases small dense low-density lipoproteins in healthy men: a randomized trial. Eur Heart J 31, 1633-1639 (2010).

75 Schuff-Werner, P., Fenger, S. \& Kohlschein, P. Role of lipid apheresis in changing times. Clin Res Cardiol Suppl 7, 7-14, doi:10.1007/s11789-012-0049-3 (2012).

76 Goldstein, J. L. \& Brown, M. S. Regulation of low-density lipoprotein receptors: implications for pathogenesis and therapy of hypercholesterolemia and atherosclerosis. Circulation 76, 504-507 (1987).

77 Revell, S. P. et al. Liver transplantation for homozygous familial hypercholesterolaemia. Arch Dis Child 73, 456-458 (1995).

78 Wierzbicki, A. S. The role of lipid lowering in transplantation. Int J Clin Pract 53, 54-59 (1999).

79 Youngblom, E. \& Knowles, J. W. Familial Hypercholesterolemia. Gene Reviews (2014). 
80 Mansoorian, M. et al. Liver transplantation as a definitive treatment for familial hypercholesterolemia: A series of 36 cases. Pediatr Transplant 19, 605-611, doi:10.1111/petr.12562 (2015).

81 Starzl, T. E. et al. Heart-liver transplantation in a patient with familial hypercholesterolaemia. Lancet 1, 1382-1383 (1984).

82 Bilheimer, D. W., Goldstein, J. L., Grundy, S. M., Starzl, T. E. \& Brown, M. S. Liver transplantation to provide low-density-lipoprotein receptors and lower plasma cholesterol in a child with homozygous familial hypercholesterolemia. $N$ Engl J Med. 311, 1658-1664, doi:10.1056/NEJM198412273112603 (1984).

83 Figuera, D. et al. Combined transplantation of heart and liver from two different donors in a patient with familial type IIa hypercholesterolemia. J Heart Transplant 5, 327-329 (1986).

84 Hibbitt, O. C. et al. Delivery and long-term expression of a $135 \mathrm{~kb}$ LDLR genomic DNA locus in vivo by hydrodynamic tail vein injection. $J$ Gene Med $\mathbf{9}$, 488-497, doi:10.1002/jgm.1041 (2007).

85 Oka, K. et al. Long-term stable correction of low-density lipoprotein receptordeficient mice with a helper-dependent adenoviral vector expressing the very lowdensity lipoprotein receptor. Circulation 103, 1274-1281 (2001).

86 Takahashi, S., Ohsugi, K., Yamamoto, T., Shiomi, M. \& Sakuragawa, N. A novel approach to ex vivo gene therapy for familial hypercholesterolemia using human amniotic epithelial cells as a transgene carrier. Tohoku J Exp Med 193, 279-292 (2001). 
87 Chowdhury, J. R. et al. Long-term improvement of hypercholesterolemia after ex vivo gene therapy in LDLR-deficient rabbits. Science 254, 1802-1805 (1991).

88 Okura, H. et al. Transplantation of human adipose tissue-derived multilineage progenitor cells reduces serum cholesterol in hyperlipidemic Watanabe rabbits. Tissue Eng Part C Methods 17, 145-154, doi:10.1089/ten.TEC.2010.0139 (2011).

89 Fattahi, F. et al. Disease-corrected hepatocyte-like cells from familial hypercholesterolemia-induced pluripotent stem cells. Mol Biotechnol 54, 863-873, doi:10.1007/s12033-012-9635-3 (2013).

90 Heyde, M. et al. Gene therapy used for tissue engineering applications. J Pharm Pharmacol 59, 329-350, doi:10.1211/jpp.59.3.0002 (2007).

91 Zhang, X. \& Godbey, W. T. Viral vectors for gene delivery in tissue engineering. Adv Drug Deliv Rev 58, 515-534, doi:10.1016/j.addr.2006.03.006 (2006).

92 Savulescu, J. Harm, ethics committees and the gene therapy death. J Med Ethics 27, 148-150 (2001).

93 Buchschacher, G. L. Introduction to retroviruses and retroviral vectors. Somat Cell Mol Genet 26, 1-11 (2001).

$94 \mathrm{Yu}, \mathrm{S}$. S. et al. Transient gene expression mediated by integrase-defective retroviral vectors. Biochem Biophys Res Commun 368, 942-947, doi:10.1016/j.bbrc.2008.02.012 (2008).

95 Hacein-Bey-Abina, S. et al. A serious adverse event after successful gene therapy for X-linked severe combined immunodeficiency. $N$ Engl $J$ Med 348, 255-256 (2003). 
96 Katz, R. A., Greger, J. G. \& Skalka, A. M. Effects of cell cycle status on early events in retroviral replication. J Cell Biochem 94, 880-889 (2005).

97 Caron, M. C. \& Caruso, M. A nuclear localization signal in the matrix of spleen necrosis virus (SNV) does not allow efficient gene transfer into quiescent cells with SNV-derived vectors. Virology 338, 292-296 (2005).

98 Roe, T., Reynolds, T. C., Yu, G. \& Brown, P. O. Integration of murine leukemia virus DNA depends on mitosis. EMBO J 12, 2099-2108. (1993).

99 Lewis, P., Hensel, M. \& Emerman, M. Human immunodeficiency virus infection of cells arrested in the cell cycle. EMBO J 11, 3053-3058 (1992).

100 Yamashita, M. \& Emerman, M. Retroviral infection of non-dividing cells: old and new perspectives. Virology 344, 88-93 (2006).

101 Grossman, M. et al. A pilot study of ex vivo gene therapy for homozygous familial hypercholesterolaemia. Nat Med 1, 1148-1154 (1995).

102 Khoo, B. Genetic therapies to lower cholesterol. Vasc Pharmacol 64, 11-15 (2015).

103 Snoeys, J. et al. Species differences in transgene DNA uptake in hepatocytes after adenoviral transfer correlate with the size of endothelial fenestrae. Gene Ther 14, 604-612, doi:10.1038/sj.gt.3302899 (2007).

104 Wisse, E. An electron microscopic study of the fenestrated endothelial lining of rat liver sinusoids. J Ultrastruct Res 31, 125-150 (1970).

105 Wisse, E. An ultrastructural characterization of the endothelial cell in the rat liver sinusoid under normal and various experimental conditions, as a contribution to 
the distinction between endothelial and Kupffer cells. J Ultrastruct Res 38, 528562 (1972).

106 Kuzmin, A. I., Finegold, M. \& Eisensmith, R. C. Macrophage depletion increases the safety, efficacy and persistence of adenovirus-mediated gene transfer in vivo. Gene Ther 4, 309-316 (1997).

107 Wolff, G. et al. Enhancement of in vivo adenovirus-mediated gene transfer and expression by prior depletion of tissue macrophages in the target organ. $J$ Virol 71, 624-629 (1997).

108 Snoeys, J. et al. Lipid emulsions potently increase transgene expression in hepatocytes after adenoviral transfer. Mol Ther 13, 98-107 (2006).

109 Verma, I. M. \& Somia, N. Gene therapy -- promises, problems and prospects. Nature 389, 239-242 (1997).

110 Smith, A. E. Viral vectors in gene therapy. Annu Rev Microbiol. 49, 807-838 (1995).

111 Khan, T. A., Sellke, F. W. \& Laham, R. J. Gene therapy progress and prospects: therapeutic angiogenesis for limb and myocardial ischemia. Gene Ther 10, 285291 (2003).

112 Qian, H. S. et al. Improved adenoviral vector for vascular gene therapy : beneficial effects on vascular function and inflammation. Circ Res 88, 911-917 (2001).

113 Wen, S., Graf, S., Massey, P. G. \& Dichek, D. A. Improved vascular gene transfer with a helper-dependent adenoviral vector. Circulation 110, 1484-1491 (2004). 
114 Fleury, S. et al. Helper-dependent adenovirus vectors devoid of all viral genes cause less myocardial inflammation compared with first-generation adenovirus vectors. Basic Res Cardiol 99, 247-256 (2004).

115 Pastore, L. et al. Helper-dependent adenoviral vector-mediated long-term expression of human apolipoprotein A-I reduces atherosclerosis in apo E-deficient mice. Gene 327, 153-160 (2004).

116 Hollinger, K. \& Chamberlain, J. S. Viral vector-mediated gene therapies. Curr Opin Neurol. 28, 522-527 (2015).

117 Chen, H. Comparative observation of the recombinant adeno-associated virus 2 using transmission electron microscopy and atomic force microscopy. Microsc Microanal 13, 384-389 (2007).

118 Odom, G. L., Gregorevic, P., Allen, J. M. \& Chamberlain, J. S. Gene therapy of mdx mice with large truncated dystrophins generated by recombination using rAAV6. Mol Ther 19, 36-45, doi:10.1038/mt.2010.205 (2011).

119 Lostal, W., Kodippili, K., Yue, Y. \& Duan, D. Full-length dystrophin reconstitution with adeno-associated viral vectors. Hum Gene Ther 25, 552-562, doi:10.1089/hum.2013.210 (2014).

120 Koo, T., Popplewell, L., Athanasopoulos, T. \& Dickson, G. Triple trans-splicing adeno-associated virus vectors capable of transferring the coding sequence for full-length dystrophin protein into dystrophic mice. Hum Gene Ther 25, 98-108, doi:10.1089/hum.2013.164 (2014). 
121 Chicoine, L. G. et al. Plasmapheresis eliminates the negative impact of AAV antibodies on microdystrophin gene expression following vascular delivery. $\mathrm{Mol}$ Ther 22, 338-347, doi:10.1038/mt.2013.244 (2014).

122 Hareendran, S. et al. Adeno-associated virus (AAV) vectors in gene therapy: immune challenges and strategies to circumvent them. Rev Med Virol 23, 399413, doi:10.1002/rmv.1762 (2013).

123 Yla-Herttuala, S. Endgame: glybera finally recommended for approval as the first gene therapy drug in the European union. Mol Ther 20, 1831-1832, doi:10.1038/mt.2012.194 (2012).

124 Manno, C. S. et al. Successful transduction of liver in hemophilia by AAV-Factor IX and limitations imposed by the host immune response. Nat Med 12, 342-347, doi:10.1038/nm1358 (2006).

125 Mingozzi, F. et al. CD8(+) T-cell responses to adeno-associated virus capsid in humans. Nat Med 13, 419-422, doi:10.1038/nm1549 (2007).

126 Rogers, G. L. et al. Innate Immune Responses to AAV Vectors. Front Microbiol 2, 194, doi:10.3389/fmicb.2011.00194 (2011).

127 Kassim, S. H. et al. Adeno-associated virus serotype 8 gene therapy leads to significant lowering of plasma cholesterol levels in humanized mouse models of homozygous and heterozygous familial hypercholesterolemia. Hum Gene Ther 24, 19-26, doi:10.1089/hum.2012.108 (2013).

128 Narsinh, K. H. \& Wu, J. C. Gene correction in human embryonic and induced pluripotent stem cells: promises and challenges ahead. Mol Ther 18, 1061-1063, doi:10.1038/mt.2010.92 (2010). 
129 Kozarsky, K. F., Jooss, K., Donahee, M., Strauss III, J. F. \& Wilson, J. M. Effective treatment of familial hypercholesterolemia in the mouse model using adenovirus-mediated transfer of the VLDL receptor gene. Nature genetics 13, 5462 (1996).

130 Heeren, J., Weber, W. \& Beisiegel, U. Intracellular processing of endocytosed triglyceride-rich lipoproteins comprises both recycling and degradation. Journal of Cell Science 112, 349-359 (1999).

131 Cichon, G. et al. Non-physiological overexpression of the low density lipoprotein receptor (LDLr) gene in the liver induces pathological intracellular lipid and cholesterol storage. J Gene Med 6, 166-175, doi:10.1002/jgm.473 (2004).

132 Krieger, M., Brown, M. S. \& Goldstein, J. L. Isolation of Chinese Hamster Cell Mutants Defective in the Receptor-mediated Endocytosis of Low Density Lipoprotein. J Mol Biol 150, 167-184 (1981).

133 Le Douarin, G., Le Douarin, N. \& Cuminge, D. Ultrastructural study of the autodifferentiation capabilities of the mesoderm of the cardiac area in the chick embryo. C R Acad Sci Hebd Seances Acad Sci D 260, 6998-7001 (1965).

134 Le Douarin, N. An experimental analysis of liver development. Med Biol. 53, 427-455 (1975).

135 Stark, K. L., McMahon, J. A. \& McMahon, A. P. FGFR-4, a new member of the fibroblast growth factor receptor family, expressed in the definitive endoderm and skeletal muscle lineages of the mouse. Development 113, 641-651 (1991). 
136 Sugi, Y., Sasse, J., Barron, M. \& Lough, J. Developmental expression of fibroblast growth factor receptor-1 (cek-1; flg) during heart development. Dev Dyn 202, 115-125 (1995).

137 Ciruna, B. G., Schwartz, L., Harpal, K., Yamaguchi, T. P. \& Rossant, J. Chimeric analysis of fibroblast growth factor receptor-1 (Fgfr1) function: a role for FGFR1 in morphogenetic movement through the primitive streak. Development 124, 2829-2841 (1997).

138 Jung, J., Zheng, M., Goldfarb, M. \& Zaret, K. S. Initiation of mammalian liver development from endoderm by fibroblast growth factors. Science 284, 19982003 (1999).

139 Crossley, P. H. \& Martin, G. R. The mouse Fgf8 gene encodes a family of polypeptides and is expressed in regions that direct outgrowth and patterning in the developing embryo. Development 121, 439-451 (1995).

140 Duncan, S. A. Mechanisms controlling early development of the liver. Mech Dev. 120, 19-33 (2003).

141 Deutsch, G., Jung, J., Zheng, M., Lóra, J. \& Zaret, K. S. A bipotential precursor population for pancreas and liver within the embryonic endoderm. Development 128, 871-881 (2001).

142 Rossi, J. M., Dunn, N. R., Hogan, B. L. \& Zaret, K. S. Distinct mesodermal signals, including BMPs from the septum transversum mesenchyme, are required in combination for hepatogenesis from the endoderm. Genes Dev 15, 1998-2009 (2001). 
143 Singh, V. Textbook of Clinical Embryology. 140-168 (Reed Elsevier India Private Limited, 2012).

144 Carlson, B. M. Human Embryology \& Developmental Biology, 5th Ed., (Saunders, 2013).

145 Kuntz, E. \& Kuntz, H.-D. Hepatology, Principles and Practice: History, Morphology, Diagnostics, Clinic, Therapy, 3rd Ed. 3 edn, (Springer, 2005).

146 Lalor, P. F., Lai, W. K., Curbishley, S. M., Shetty, S. \& Adams, D. H. Human hepatic sinusoidal endothelial cells can be distinguished by expression of phenotypic markers related to their specialised functions in vivo. World $J$ Gastroenterol 12, 5429-5439 (2006).

147 Jenne, C. N. \& Kubes, P. Immune surveillance by the liver. Nat Immunol 14, 9961006, doi:10.1038/ni.2691 (2013).

148 Straub, A. C. et al. Arsenic-stimulated liver sinusoidal capillarization in mice requires NADPH oxidase-generated superoxide. J Clin Invest 118, 3980-3989, doi:10.1172/JCI35092 (2008).

149 Huet, P.-M., Goresky, C. A., Villenueve, J.-P., Marleau, D. \& Lough, J. O. Assessment of Liver Microcirculation in Human Cirrhosis. J Clin Invest 70, 12341244 (1982).

150 Kordes, C. et al. CD133+ hepatic stellate cells are progenitor cells. Biochem Biophys Res Commun 352, 410-417, doi:10.1016/j.bbrc.2006.11.029 (2007).

151 Hindley, C. J., Mastrogiovanni, G. \& Huch, M. The plastic liver: differentiated cells, stem cells, every cell? J Clin Invest 124, 5099-5102 (2014). 
152 Yang, L. et al. Fate-mapping evidence that hepatic stellate cells are epithelial progenitors in adult mouse livers. Stem Cells 26, 2104-2113, doi:10.1634/stemcells.2008-0115 (2008).

153 CDC. Chronic Liver Disease and Cirrhosis, $<$ http://www.cdc.gov/nchs/fastats/liver-disease.htm> (2015).

154 ALF. Liver

Transplant, $<$ http://www.liverfoundation.org/abouttheliver/info/transplant/> (2015).

155 Allen, J. W., Hassanein, T. \& Bhatia, S. N. Advances in bioartificial liver devices. Hepatology 34, 447-455 (2001).

156 Mitaka, T. The current status of primary hepatocyte culture. Int J Exp Pathol 79, 393-409 (1998).

157 Shulman, M. \& Nahmias, Y. Long-term culture and coculture of primary rat and human hepatocytes. Methods Mol Biol 945, 287-302, doi:10.1007/978-1-62703125-7_17 (2013).

158 Kubota, H. \& Reid, L. M. Clonogenic hepatoblasts, common precursors for hepatocytic and biliary lineages, are lacking classical major histocompatibility complex class I antigen. Proc Natl Acad Sci U S A 97, 12132-12137 (2000).

159 Isom, H. C., Secott, T., Georgoff, I., Woodworth, C. \& Mummaw, J. Maintenance of differentiated rat hepatocytes in primary culture. Proc Natl Acad Sci U S A 82, 3252-3256 (1985).

160 Dich, J., Vind, C. \& Grunnet, N. Long-term culture of hepatocytes: effect of hormones on enzyme activities and metabolic capacity. Hepatology 8, 39-45 (1988). 
161 Kelly, J. H. \& Darlington, G. J. Modulation of the liver specific phenotype in the human hepatoblastoma line Hep G2. In Vitro Cell Dev Biol 25, 217-222 (1989).

162 Nyberg, S. L. \& Misra, S. P. Hepatocyte liver-assist systems--a clinical update. Mayo Clin Proc 73, 765-771 (1998).

163 Werner, A. et al. Cultivation of immortalized human hepatocytes HepZ on macroporous CultiSpher G microcarriers. Biotechnol Bioeng 68, 59-70 (2000).

164 Kono, Y., Yang, S., Letarte, M. \& Roberts, E. A. Establishment of a human hepatocyte line derived from primary culture in a collagen gel sandwich culture system. Exp Cell Res 221, 478-485 (1995).

165 Gripon, P. et al. Infection of a human hepatoma cell line by hepatitis B virus. Proc Natl Acad Sci U S A 99, 15655-15660 (2002).

166 Parent, R., Marion, M. J., Furio, L., Trepo, C. \& Petit, M. A. Origin and characterization of a human bipotent liver progenitor cell line. Gastroenterology 126, 1147-1156 (2004).

167 Chen, S. C. et al. Clinical experience with a porcine hepatocyte-based liver support system. Int J Artif Organs 19, 664-669 (1996).

168 Morsiani, E. et al. Early experiences with a porcine hepatocyte-based bioartificial liver in acute hepatic failure patients. Int J Artif Organs 25, 192-202 (2002).

169 van de Kirkhove, M. P., Hoekstra, R., Chamuleau, R. A. \& van Gulik, T. M. Clinical application of bioartificial liver support systems. Ann Surg 240, 216-230 (2004). 
170 Demetriou, A. A. et al. Prospective, Randomized, Multicenter, Controlled Trial of a Bioartificial Liver in Treating Acute Liver Failure. Annals of Surgery 239, 660670, doi:10.1097/01.sla.0000124298.74199.e5 (2004).

171 Vannier, P. \& Carriolet, R. Xenografts and the probability of viral zoonotic risk: reflections based on the sanitary control of a pig population free from specific pathogenic organisms and risk analysis. Pathol Biol 48, 389-394 (2000).

172 Boneva, R. S., Folks, T. M. \& Chapman, L. E. Infectious disease issues in xenotransplantation. Clin Microbiol Rev 14, 1-14, doi:10.1128/CMR.14.1.1$14.2001(2001)$.

173 Schrem, H., Kleine, M., Borlak, J. \& Klempnauer, J. Physiological incompatibilities of porcine hepatocytes for clinical liver support. Liver Transpl 12, 1832-1840, doi:10.1002/1t.20918 (2006).

174 Kelly, S. J. Studies of the developmental potential of four- and eight-cell stage mouse blastomeres. $J$ Exp Zool 200, 365-376 (1977).

175 Stadtfeld, M. \& Hochedlinger, K. Induced pluripotency: history, mechanisms, and applications. Genes Dev 24, 2239-2263, doi:10.1101/gad.1963910 (2010).

176 Briggs, R. \& King, T. J. Transplantation of living nuclei from blastula cells into enucleated frogs' eggs. Proc Natl Acad Sci U S A 38, 455-463 (1952).

177 King, T. J. \& Briggs, R. Changes in the nuclei of differentiating gastrula cells, as demonstrated by nuclear transplantation. Proc Natl Acad Sci U S A 41 (1955).

178 Gurdon, J. B., Byrne, J. A. \& Simonsson, S. Nuclear reprogramming and stem cell creation. Proc Natl Acad Sci U S A 100, 11819-11822 (2003). 
179 Wakayama, T. \& Yanagimachi, R. Cloning of male mice from adult tail-tip cells. Nat Genet 22, 127-128 (1999).

180 Tamashiro, K. L. et al. Cloned mice have an obese phenotype not transmitted to their offspring. Nat Med 8, 262-267 (2002).

181 Stevens, L. C. \& Little, C. C. Spontaneous testicular teratomas in an inbred strain of mice. Proc Natl Acad Sci US A 40, 1080-1087 (1954).

182 Kleinsmith, L. J. \& Pierce, G. B. Multipotentiality of single embryonal carcinoma cells. Cancer Res 24, 1544-1551 (1964).

183 Kahan, B. W. \& Ephrussi, B. Developmental potentialities of clonal in vitro cultures of mouse testicular teratoma. J Natl Cancer Inst 44 (1970).

184 Miller, R. A. \& Ruddle, F. H. Pluripotent teratocarcinoma-thymus somatic cell hybrids. Cell 9, 45-55 (1976).

185 Miller, R. A. \& Ruddle, F. H. Properties of teratocarcinoma-thymus somatic cell hybrids. Somat Cell Genet 3, 247-261 (1977).

186 Solter, D., Skreb, N. \& Damjanov, I. Extrauterine growth of mouse egg-cylinders results in malignant teratoma. Nature 227, 503-504 (1970).

187 Evans, M. J. \& Kaufman, M. H. Establishment in culture of pluripotential cells from mouse embryos. Nature 292, 154-156 (1981).

188 Martin, G. R. Isolation of a pluripotent cell line from early mouse embryos cultured in medium conditioned by terato-carcinoma stem cells. Proc Natl Acad Sci U S A 78, 7634-7638 (1981). 
189 Andrews, P. W. et al. Pluripotent embryonal carcinoma clones derived from the human teratocarcinoma cell line Tera-2. Differentiation in vivo and in vitro. Lab Invest 50, 147-162 (1984).

190 Andrews, P. W. Retinoic acid induces neuronal differentiation of a cloned human embryonal carcinoma cell line in vitro. Dev Biol 103, 285-293 (1984).

191 Pera, M. F., Cooper, S., Mills, J. \& Parrington, J. M. Isolation and characterization of a multipotent clone of human embryonal carcinoma cells. Differentiation 42, 10-23 (1989).

192 Bongso, T. A., Fong, C. Y., Ng, C. Y. \& Ratnam, S. S. Blastocyst transfer in human in vitro fertilization: the use of embryo co-culture. Cell Biol Int 18, 11811189 (1994).

193 Thomson, J. A. et al. Isolation of a primate embryonic stem cell line. Proc Natl Acad Sci U S A 92, 7844-7848 (1995).

194 Thomson, J. A. et al. Pluripotent cell lines derived from common marmoset (Callithrix jacchus) blastocysts. Biol Reprod 55, 254-259 (1996).

195 Thomson, J. A. et al. Embryonic stem cell lines derived from human blastocysts. Science 282, 1145-1147 (1998).

196 Stice, S. L. et al. Human embryonic stem cells: challenges and opportunities. Repro Fert Dev 18, 839-846 (2006).

197 EuroStemCell. Embryonic stem cell research: an ethical dilemma, $<$ http://www.eurostemcell.org/factsheet/embyronic-stem-cell-research-ethicaldilemma> (2011). 
198 Davis, R. L., Weintraub, H. \& Lassar, A. B. Expression of a single transfected cDNA converts fibroblasts to myoblasts. Cell 51, 987-1000 (1987).

199 Xie, H., Ye, M., Feng, R. \& Graf, T. Stepwise reprogramming of B cells into macrophages. Cell 117, 663-676 (2004).

200 Laiosa, C. V., Stadtfeld, M., Xie, H., de Andres-Aguayo, L. \& Graf, T. Reprogramming of committed $\mathrm{T}$ cell progenitors to macrophages and dendritic cells by C/EBP alpha and PU.1 transcription factors. Immunity 25, 731-744 (2006).

201 Tada, M., Tada, T., Lefebvre, L., Barton, S. C. \& Surani, M. A. Embryonic germ cells induce epigenetic reprogramming of somatic nucleus in hybrid cells. EMBO $J 16,6510-6520$ (1997).

202 Tada, M., Takahama, Y., Abe, K., Nakatsuji, N. \& Tada, T. Nuclear reprogramming of somatic cells by in vitro hybridization with ES cells. Curr Biol. 11, 1553-1558 (2001).

203 Tokuzawa, Y. et al. Fbx15 is a novel target of Oct3/4 but is dispensable for embryonic stem cell self-renewal and mouse development. Mol Cell Biol 23, 2699-2708 (2003).

204 Takahashi, K. \& Yamanaka, S. Induction of pluripotent stem cells from mouse embryonic and adult fibroblast cultures by defined factors. Cell 126, 663-676, doi:10.1016/j.cell.2006.07.024 (2006).

205 Takahashi, K. et al. Induction of pluripotent stem cells from adult human fibroblasts by defined factors. Cell 131, 861-872, doi:10.1016/j.cell.2007.11.019 (2007). 
206 Giorgetti, A. et al. Generation of induced pluripotent stem cells from human cord blood using OCT4 and SOX2. Cell Stem Cell 5, 353-357, doi:10.1016/j.stem.2009.09.008 (2009).

207 Kim, J. B. et al. Pluripotent stem cells induced from adult neural stem cells by reprogramming with two factors. Nature 454, 646-650, doi:10.1038/nature07061 (2008).

208 Tsai, S. Y. et al. Oct4 and klf4 reprogram dermal papilla cells into induced pluripotent stem cells. Stem Cells 28, 221-228, doi:10.1002/stem.281 (2010).

209 Utikal, J., Maherali, N., Kulalert, W. \& Hochedlinger, K. Sox2 is dispensable for the reprogramming of melanocytes and melanoma cells into induced pluripotent stem cells. J Cell Sci 122, 3502-3510 (2009).

210 Yu, J. et al. Induced Pluripotent Stem Cell Lines Derived from Human Somatic Cells. Sciencexpress, 1-4 (2007).

211 Lei, H. et al. De novo DNA cytosine methyltransferase activities in mouse embryonic stem cells. Development 122, 3195-3205 (1996).

212 Matsui, T. et al. Proviral silencing in embryonic stem cells requires the histone methyltransferase ESET. Nature 464, 927-931 (2010).

213 Okita, K., Ichisaka, T. \& Yamanaka, S. Generation of germline-competnet induced pluripotent stem cells. Nature 448, 313-317 (2007).

214 Brambrink, T. et al. Sequential expression of pluripotency markers during direct reprogramming of mouse somatic cells. Cell Stem Cell 2, 151-159 (2008). 
215 Sommer, C. A. et al. Excision of reprogramming transgenes improves the differentiation potential of iPS cells generated with a single excisable vector. . Stem Cells 28, 64-74 (2010).

216 Stadtfeld, M., Maherali, N., Breault, D. T. \& Hochedlinger, K. Defining molecular cornerstones during fibroblast to iPS cell reprogramming in mouse. Cell Stem Cell 2, 230-240 (2008).

217 Hockemeyer, D. et al. Efficient targeting of expressed and silent genes in human ESCs and iPSCs using zinc-finger nucleases. Nat Biotechnol 27, 851-857, doi:10.1038/nbt.1562 (2009).

218 Maherali, N. et al. A high-efficiency system for the generation and study of human induced pluripotent stem cells. Cell Stem Cell 3, 340-345 (2008).

219 Wernig, M. et al. A drug- inducible transgenic system for direct reprogramming of mul- tiple somatic cell types. Nat Biotechnol 26, 916-924 (2008).

220 Woltjen, K. et al. piggyBac transposition reprograms fibroblasts to induced pluripotent stem cells. . Nature 458, 766-770 (2009).

221 Zhou, W. \& Freed, C. R. Adenoviral gene delivery can repro- gram human fibroblasts to induced pluripotent stem cells. Stem Cells 27, 2667-2674 (2009).

222 Fusaki, N., Ban, H., Nishiyama, A., Saeki, K. \& Hasegawa, M. Efficient induction of transgene-free human pluripotent stem cells using a vector based on Sendai virus, an RNA virus that does not integrate into the host genome. . Proc Jpn Acad 85, 348-362 (2009).

223 Jia, F. et al. A nonviral minicircle vector for deriving human iPS cells. Nat Methods 7, 197-199, doi:10.1038/nmeth.1426 (2010). 
$224 \mathrm{Yu}$, J. et al. Human induced pluripotent stem cells free of vector and transgene sequences. . Science 324, 797-801 (2009).

225 Soldner, F. et al. Parkinson's disease patient-derived induced pluripotent stem cells free of viral reprogramming factors. Cell 136, 964-977, doi:10.1016/j.cell.2009.02.013 (2009).

226 Chang, C. W. et al. Polycistronic lentiviral vector for 'hit and run' reprogramming of adult skin fibroblasts to induced pluripotent stem cells. . Stem Cells 27, 10421049 (2009).

227 Wilson, M. H., Coates, C. J. \& George, A. L. PiggyBac Transposon-mediated Gene Transfer in Human Cells. Mol Ther 15, 139-145 (2007).

228 Kaji, K. et al. Virus-free induction of pluripotency and subsequent excision of reprogramming factors. Nature $\mathbf{4 5 8}, 771-775$ (2009).

229 Cho, H. J. et al. Induction of pluripotent stem cells from adult somatic cells by protein-based repro- gramming without genetic manipulation. Blood 116, 386-395 (2010).

230 Warren, L. et al. Highly efficient reprogramming to pluripotency and directed differentiation of human cells with synthetic modified mRNA. Cell Stem Cell 7, 618-630, doi:10.1016/j.stem.2010.08.012 (2010).

231 Yakubov, E., Rechavi, G., Rozenblatt, S. \& Givol, D. Reprogramming of human fibroblasts to pluripotent stem cells using mRNA of four transcription factors. Biochem Biophys Res Commun 394, 189-193, doi:10.1016/j.bbrc.2010.02.150 (2010). 
232 Bernal, J. A. RNA-based tools for nuclear reprogramming and lineageconversion: towards clinical applications. J Cardiovasc Transl Res 6, 956-968, doi:10.1007/s12265-013-9494-8 (2013).

233 Hussein, S. M. \& Nagy, A. A. Progress made in the reprogramming field: new factors, new strategies and a new outlook. Curr Opin Genet Dev 22, 435-443, doi:10.1016/j.gde.2012.08.007 (2012).

234 Gonzalez, F., Boue, S. \& Izpisua Belmonte, J. C. Methods for making induced pluripotent stem cells: reprogramming a la carte. Nat Rev Genet 12, 231-242, doi:10.1038/nrg2937 (2011).

235 Sullivan, G. J., Bai, Y., Fletcher, J. \& Wilmut, I. Induced pluripotent stem cells: epigenetic memories and practical implications. Mol Hum Reprod 16, 880-885, doi:10.1093/molehr/gaq091 (2010).

236 Bilic, J. \& Izpisua Belmonte, J. C. Concise review: Induced pluripotent stem cells versus embryonic stem cells: close enough or yet too far apart? Stem Cells 30, 3341 (2012).

237 Maherali, N. et al. Directly reprogrammed fibroblasts show global epigenetic remodeling and widespread tissue contribution. Cell Stem Cell 1, 55-70 (2007).

238 Wernig, M. et al. In vitro reprogramming of fibroblasts into a pluripotent ES-celllike state. Nature 448, 318-324 (2007).

239 Mikkelsen, T. S. et al. Dissecting direct reprogramming through integrative genomic analysis. Nature 454, 49-55, doi:10.1038/nature07056 (2008). 
240 Feng, Q. et al. Hemangioblastic derivatives from human induced pluripotent stem cells exhibit limited expansion and early senescence. Stem Cells 28, 704-712, doi:10.1002/stem.321 (2010).

241 Chin, M. H. et al. Induced pluripotent stem cells and embryonic stem cells are distinguished by gene expression signatures. Cell Stem Cell 5, 111-123, doi:10.1016/j.stem.2009.06.008 (2009).

242 Wilson, K. D. et al. MicroRNA profiling of human-induced pluripotent stem cells. Stem Cells Dev 18, 749-758, doi:10.1089/scd.2008.0247 (2009).

$243 \mathrm{Hu}, \mathrm{B}$. Y. et al. Neural differentiation of human induced pluripotent stem cells follows developmental principles but with variable potency. Proc Natl Acad Sci U S A 107, 4335-4340, doi:10.1073/pnas.0910012107 (2010).

244 Kim, K. et al. Epigenetic memory in induced pluripotent stem cells. Nature 467, 285-290, doi:10.1038/nature09342 (2010).

$245 \mathrm{Ji}, \mathrm{H}$. et al. Comprehensive methylome map of lineage commitment from haematopoietic progenitors. Nature 467, 338-342, doi:10.1038/nature09367 (2010).

246 Polo, J. M. et al. Cell type of origin influences the molecular and functional properties of mouse induced pluripotent stem cells. Nat Biotechnol 28, 848-855, doi:10.1038/nbt.1667 (2010).

247 Newman, A. M. \& Cooper, J. B. Lab-specific gene expression signatures in pluripotent stem cells. Cell Stem Cell 7, 258-262, doi:10.1016/j.stem.2010.06.016 (2010). 
248 Osafune, K. et al. Marked differences in differentiation propensity among human embryonic stem cell lines. Nat Biotechnol 26, 313-315, doi:10.1038/nbt1383 (2008).

249 Tavakoli, T. et al. Self-renewal and differentiation capabilities are variable between human embryonic stem cell lines I3, I6 and BG01V. BMC Cell Biol 10, 44, doi:10.1186/1471-2121-10-44 (2009).

250 Gutierrez-Aranda, I. et al. Human induced pluripotent stem cells develop teratoma more efficiently and faster than human embryonic stem cells regardless the site of injection. Stem Cells 28, 1568-1570, doi:10.1002/stem.471 (2010).

251 Lowry, W. E. \& Quan, W. L. Roadblocks en route to the clinical application of induced pluripotent stem cells. J Cell Sci 123, 643-651, doi:10.1242/jcs.054304 (2010).

252 Ben-David, U. \& Benvenisty, N. Chemical ablation of tumor-initiating human pluripotent stem cells. Nat Protoc 9, 729-740, doi:10.1038/nprot.2014.050 (2014).

253 Ben-David, U. et al. Selective elimination of human pluripotent stem cells by an oleate synthesis inhibitor discovered in a high-throughput screen. Cell Stem Cell 12, 167-179, doi:10.1016/j.stem.2012.11.015 (2013).

254 Wu, C. et al. Development of an inducible caspase-9 safety switch for pluripotent stem cell-based therapies. Mol Ther Methods Clin Dev 1, 14053, doi:10.1038/mtm.2014.53 (2014).

255 Nakagawa, M. et al. Generation of induced pluripotent stem cells without Myc from mouse and human fibroblasts. Nat Biotechnol 26, 101-106, doi:10.1038/nbt1374 (2008). 
256 Judson, R. L., Babiarz, J. E., Venere, M. \& Blelloch, R. Embryonic stem cellspecific microRNAs promote induced pluripotency. Nat Biotechnol 27, 459-461, doi:10.1038/nbt.1535 (2009).

257 Uren, A. G., Kool, J., Berns, A. \& van Lohuizen, M. Retroviral insertional mutagenesis: past, present and future. Oncogene 24, 7656-7672 (2005).

258 Okita, K. et al. A more efficient method to generate integration-free human iPS cells. Nat Methods 8, 409-412 (2011).

259 Okita, K., Hong, H., Takahashi, K. \& Yamanaka, S. Generation of mouse-induced pluripotent stem cells with plasmid vectors. Nat Protoc 5, 418-428, doi:10.1038/nprot.2009.231 (2010).

260 Huang, B. et al. A virus-free poly-promoter vector induces pluripotency in quiescent bovine cells under chemically defined conditions of dual kinase inhibition. PLoS One 6 (2011).

261 Yu, J., Chau, K. F., Vodyanik, M. A., Jiang, J. \& Jiang, Y. Efficient feeder-free episomal reprogramming with small molecules. PLoS One 6, e17557, doi:10.1371/journal.pone.0017557 (2011).

262 Puppi, J. et al. Improving the techniques for human hepatocyte transplantation: report from a consensus meeting in London. Cell Transplant 21, 1-10, doi:10.3727/096368911X566208 (2012).

263 Liu, L. et al. The microenvironment in hepatocyte regeneration and function in rats with advanced cirrhosis. Hepatology 55, 1529-1539, doi:10.1002/hep.24815 (2012). 
264 Hoppo, T., Komori, J., Manohar, R., Stolz, D. B. \& Lagasse, E. Rescue of lethal hepatic failure by hepatized lymph nodes in mice. Gastroenterology 140, 656-666 e652, doi:10.1053/j.gastro.2010.11.006 (2011).

265 Song, Z. et al. Efficient generation of hepatocyte-like cells from human induced pluripotent stem cells. Cell Res 19, 1233-1242, doi:10.1038/cr.2009.107 (2009).

266 Si-Tayeb, K. et al. Highly efficient generation of human hepatocyte-like cells from induced pluripotent stem cells. Hepatology 51, 297-305, doi:10.1002/hep.23354 (2010).

267 Ishikawa, T. et al. Human induced hepatic lineage-oriented stem cells: autonomous specification of human iPS cells toward hepatocyte-like cells without any exogenous differentiation factors. PLoS One 10, e0123193, doi:10.1371/journal.pone.0123193 (2015).

268 Takebe, T. et al. Vascularized and functional human liver from an iPSC-derived organ bud transplant. Nature 499, 481-484, doi:10.1038/nature12271 (2013).

269 Zaret, K. S. \& Grompe, M. Generation and regeneration of cells of the liver and pancreas. Science 322, 1490-1494, doi:10.1126/science.1161431 (2008).

270 Cayo, M. A. et al. JD induced pluripotent stem cell-derived hepatocytes faithfully recapitulate the pathophysiology of familial hypercholesterolemia. Hepatology 56, 2163-2171, doi:10.1002/hep.25871 (2012).

271 Basma, H. et al. Differentiation and transplantation of human embryonic stem cell-derived hepatocytes. Gastroenterology 136, 990-999, doi:10.1053/j.gastro.2008.10.047 (2009). 
272 Schwartz, R. E., Fleming, H. E., Khetani, S. R. \& Bhatia, S. N. Pluripotent stem cell-derived hepatocyte-like cells. Biotechnol Adv 32, 504-513, doi:10.1016/j.biotechadv.2014.01.003 (2014).

273 Purves, W. K., Orians, G. H. \& Heller, H. C. Life: The Science of Biology, 4th Ed., (Sinauer Associates, 1994).

274 Schmidt-Lademann, S., Stoeter, P. \& Voigt, K. Embryonal and fetal development of capillaries: microangiographic investigations. Diagn Imaging 49, 177-187 (1980).

275 Stoeter, P., Schmidt-Lademann, S. \& Voigt, K. Embryonal and fetal development of capillaries: microangiographic investigations. Diagn Imaging 49, 131-140 (1980).

276 Penn, J. Retinal and Choroidal Angiogenesis. 1 edn, 119 (Springer, 2008).

277 Pavelka, M. \& Roth, J. Functional Ultrastructure. 1 edn, 232 (Springer-Verlag, 2005).

278 Aschner, J. L., Lum, H., Fletcher, P. W. \& Malik, P. B. Bradykinin- and thrombin-induced increases in endothelial permeability occur independently of phospholipase $\mathrm{C}$ but require protein kinase C activation. J Cell Physiol 173, 387396 (1997).

279 Corson, M. A. et al. Phosphorylation of endothelial nitric oxide synthase in response to fluid shear stress. Circ Res 79, 984-991 (1996).

280 Govers, R. \& Rabelink, T. J. Cellular regulation of endothelial nitric oxide synthase. Am J Physiol Renal Physiol 280, F193-206 (2001). 
281 Moncada, S. \& Erusalimsky, J. D. Does nitric oxide modulate mitochondrial energy generation and apoptosis? Nat Rev Mol Cell Biol 3, 214-220 (2002).

282 Gimbrone, M. A. Vascular endothelium, hemodynamic forces, and atherogenesis. Am J Pathol 155, 1-5 (1999).

283 Deanfield, J. E., Halcox, J. P. \& Rabelink, T. J. Endothelial function and dysfunction: testing and clinical relevance. Circulation 115, 1285-1295 (2007).

284 Ghosh, S. \& Karin, M. Missing pieces in the NF-kappaB puzzle. Cell 109, S81-96 (2002).

285 Kinlay, S. et al. Role of endothelin-1 in the active constriction of human atherosclerotic coronary arteries. Circulation 104, 1114-1118 (2001).

286 Moncada, S., Higgs, E. A. \& Vane, J. R. Human arterial and venous tissues generate prostacyclin (prostaglandin $\mathrm{x}$ ), a potent inhibitor of platelet aggregation. Lancet 1, 18-20 (1977).

287 Alberts, B. et al. Molecular Biology of the Cell. 6 edn, 1464 (Garland Science, 2014).

288 Folkman, J. \& Haudenschild, C. Angiogenesis in vitro. Nature 288, 551-556 (1980).

289 Madri, J. A. \& Williams, S. K. Capillary Endothelial Cell Cultures: Phenotypic Modulation by Matrix Components. J Cell Biol 97, 153-165 (1983).

290 Montesano, R., Orci, L. \& Vassalli, P. In vitro rapid organization of endothelial cells into capillary-like networks is promoted by collagen matrices. J Cell Biol $\mathbf{9 7}$, 1648-1652 (1983). 
291 Vogt, M. T. \& Farber, E. On the molecular pathology of ischemic renal cell death. Reversible and irreversible cellular and mitochondrial metabolic alterations. $A m J$ Pathol 53, 1-26 (1968).

292 Cammermeyer, J. "Ischemic neuronal disease" of Spielmeyer. A reevaluation. Arch Neurol 29, 391-393 (1973).

293 Jennings, R. B. \& Ganote, C. E. Structural changes in myocardium during acute ischemia. Circ Res 35, 156-172 (1974).

294 Jones, R. T. \& Trump, B. F. Cellular and subcellular effects of ischemia on the pancreatic acinar cell: in vitro studies of rat tissue. Virchow's Arch B Cell Pathol 19, 325-336 (1975).

295 Delva, E. et al. Vascular occlusions for liver resections. Operative management and tolerance to hepatic ischemia: 142 cases. Ann Surg 209, 211-218 (1989).

296 Griffith, L. G. \& Naughton, G. Tissue engineering--current challenges and expanding opportunities. Science 295, 1009-1014 (2002).

297 Cardinal, K. O., Bonnema, G. T., Hofer, H., Kehlet Barton, J. \& Williams, S. K. Tissue-Engineered Vascular Grafts as In Vitro Blood Vessel Mimics for the Evaluations of Endothelialization of Intravascular Devices. Tissue Eng Part A 12, 3431-3438 (2006).

298 Traktuev, D. O. et al. A population of multipotent CD34-positive adipose stromal cells share pericyte and mesenchymal surface markers, reside in a periendothelial location, and stabilize endothelial networks. Circ Res 102, 77-85, doi:10.1161/CIRCRESAHA.107.159475 (2008). 
299 Koh, Y. J. et al. Stromal vascular fraction from adipose tissue forms profound vascular network through the dynamic reassembly of blood endothelial cells. Arterioscler Thromb Vasc Biol 31, 1141-1150, doi:10.1161/ATVBAHA.110.218206 (2011).

300 Merfeld-Clauss, S. et al. Adipose stromal cells differentiate along a smooth muscle lineage pathway upon endothelial cell contact via induction of activin A. Circ Res 115, 800-809, doi:10.1161/CIRCRESAHA.115.304026 (2014).

301 Leblanc, A. J., Touroo, J. S., Hoying, J. B. \& Williams, S. K. Adipose stromal vascular fraction cell construct sustains coronary microvascular function after acute myocardial infarction. Am J Physiol Heart Circ Physiol 302, H973-982, doi:10.1152/ajpheart.00735.2011 (2012).

302 Premaratne, G. U. et al. Stromal vascular fraction transplantation as an alternative therapy for ischemic heart failure: anti-inflammatory role. J Cardiothorac Surg 6, 43, doi:10.1186/1749-8090-6-43 (2011).

303 Rehman, J. et al. Secretion of angiogenic and antiapoptotic factors by human adipose stromal cells. Circulation 109, 1292-1298 (2004).

304 Cervelli, V. et al. Application of enhanced stromal vascular fraction and fat grafting mixed with PRP in post-traumatic lower extremity ulcers. Stem Cell Res 6, 103-111, doi:10.1016/j.scr.2010.11.003 (2011).

305 Rodbell, M. Metabolism of Isolated Fat Cells. J Biol Chem 239, 375-380 (1964).

306 Rodbell, M. \& Jones, A. B. Metabolism of Isolated Fat Cells. J Biol Chem 241, 140-142 (1966). 
307 Rodbell, M. The Metabolism of Isolated Fat Cells. J Biol Chem 241, 3909-3917 (1966).

308 Wagner, R. C. \& Matthews, M. A. The isolation and culture of capillary endothelium from epididymal fat. Microvasc Res 10, 286-297 (1975).

309 Van, R. L., Bayliss, C. E. \& Roncari, D. A. Cytological and enzymological characterization of adult human adipocyte precursors in culture. $J$ Clin Invest 58, 699-704 (1976).

310 Takahashi, K., Goto, T., Mukai, K., Sawasaki, Y. \& Hata, J. Cobblestone monolayer cells from human omental adipose tissue are possibly mesothelial, not endothelial. In Vitro Cell Dev Biol 25, 109-111 (1989).

311 Williams, S. K., Wang, T. F., Castrillo, R. \& Jarrell, B. E. Liposuction-derived human fat used for vascular graft sodding contains endothelial cells and not mesothelial cells as the major cell type. J Vasc Surg. 19, 916-923 (1994).

312 Riordan, N. H. et al. Non-expanded adipose stromal vascular fraction cell therapy for multiple sclerosis. J Transl Med 7, 29, doi:10.1186/1479-5876-7-29 (2009).

313 Zimmerlin, L. et al. Stromal vascular progenitors in adult human adipose tissue. Cytometry A 77, 22-30, doi:10.1002/cyto.a.20813 (2010).

314 Gimble, J. M., Bunnell, B. A., Chiu, E. S. \& Guilak, F. Concise review: Adiposederived stromal vascular fraction cells and stem cells: let's not get lost in translation. Stem Cells 29, 749-754, doi:10.1002/stem.629 (2011).

315 Goodwin, A. M. \& D'Amore, P. A. in Tumor Angiogenesis (eds D. Marmé \& N. Fusenig) Ch. 15, (Springer-Verlag, 2008). 
316 Boyd, N. L. et al. Dissecting the role of human embryonic stem cell-derived mesenchymal cells in human umbilical vein endothelial cell network stabilization in three-dimensional environments. Tissue Eng Part A 19, 211-223, doi:10.1089/ten.tea.2011.0408 (2013).

317 Koike, N. et al. Tissue engineering: creation of long-lasting blood vessels. Nature 428, 138-139 (2004).

318 Stratman, A. N., Malotte, K. M., Mahan, R. D., Davis, M. J. \& Davis, G. E. Pericyte recruitment during vasculogenic tube assembly stimulates endothelial basement membrane matrix formation. Blood 114, 5091-5101, doi:10.1182/blood2009-05- (2009).

319 Yoshimura, K. et al. Characterization of freshly isolated and cultured cells derived from the fatty and fluid portions of liposuction aspirates. J Cell Physiol 208, 64-76, doi:10.1002/jcp.20636 (2006).

320 Eto, H. et al. Characterization of human adipose tissue-resident hematopoietic cell populations reveals a novel macrophage subpopulation with CD34 expression and mesenchymal multipotency. Stem Cells Dev 22, 985-997, doi:10.1089/scd.2012.0442 (2013).

321 Rupnick, M. A. et al. Adipose tissue mass can be regulated through the vasculature. Proc Natl Acad Sci U S A 99, 10730-10735 (2002).

322 Prunet-Marcassus, B. et al. From heterogeneity to plasticity in adipose tissues: site-specific differences. Exp Cell Res 312, 727-736 (2006).

323 Ong, W. K. \& Sugii, S. Adipose-derived stem cells: fatty potentials for therapy. Int J Biochem Cell Biol 45, 1083-1086, doi:10.1016/j.biocel.2013.02.013 (2013). 
324 Rajashekhar, G. et al. Regenerative therapeutic potential of adipose stromal cells in early stage diabetic retinopathy. PLoS One 9, e84671, doi:10.1371/journal.pone.0084671 (2014).

325 Kono, T. M. et al. Human adipose-derived stromal/stem cells protect against STZ-induced hyperglycemia: analysis of hASC-derived paracrine effectors. Stem Cells 32, 1831-1842, doi:10.1002/stem.1676 (2014).

326 Cai, L. et al. Suppression of hepatocyte growth factor production impairs the ability of adipose-derived stem cells to promote ischemic tissue revascularization. Stem Cells 25, 3234-3243, doi:10.1634/stemcells.2007-0388 (2007).

327 Crisan, M. et al. A perivascular origin for mesenchymal stem cells in multiple human organs. Cell Stem Cell 3, 301-313, doi:10.1016/j.stem.2008.07.003 (2008).

328 Amos, P. J. et al. IFATS collection: The role of human adipose-derived stromal cells in inflammatory microvascular remodeling and evidence of a perivascular phenotype. Stem Cells 26, 2682-2690, doi:10.1634/stemcells.2008-0030 (2008).

329 Zannettino, A. C. et al. Multipotential human adipose-derived stromal stem cells exhibit a perivascular phenotype in vitro and in vivo. $J$ Cell Physiol 214, 413-421 (2008).

330 Smas, C. M. \& Sul, H. S. Pref-1, a protein containing EGF-like repeats, inhibits adipocyte differentiation. Cell 73, 725-734 (1993).

331 Merfeld-Clauss, S., Gollahalli, N., March, K. L. \& Traktuev, D. O. Adipose tissue progenitor cells directly interact with endothelial cells to induce vascular network formation. Tissue Eng Part A 16, 2953-2966, doi:10.1089/ten.TEA.2009.0635 (2010). 
332 Merfeld-Clauss, S., Lupov, I. P., Lu, H., March, K. L. \& Traktuev, D. O. Adipose Stromal Cell Contact with Endothelial Cells Results in Loss of Complementary Vasculogenic Activity Mediated by Induction of Activin A. Stem Cells 33, 30393051 (2015).

333 Williams, S. K. et al. Adipose Stromal Vascular Fraction Cells Isolated Using an Automated Point of Care System Improve the Patency of Expanded Polytetrafluoroethylene Vascular Grafts. Tissue Eng Part A 19, 1295-1302 (2013).

334 Katz, A. J., Hedrick, M. H., Llull, R. \& Futrell, J. W. A novel device for the simple and efficient refinement of liposuctioned tissue. Plast Reconstr Surg 107, 595-597 (2001).

335 Brey, E. M. Vascularization: Regenerative Medicine and Tissue Engineering. (2014).

336 Fox, I. J. \& Roy-Chowdhury, J. Hepatocyte Transplantation. J Hepatol 40, 878$886(2004)$.

337 Strom, S. C. et al. Transplantation of human hepatocytes. Transplant Proc 29, 2103-2106 (1997).

338 Nagata, H. et al. Prolonged survival of porcine hepatocytes in cynomolgus monkeys. Gastroenterology 132, 321-329, doi:10.1053/j.gastro.2006.10.013 (2007).

339 Habibullah, C. M., Syed, I. H., Qamar, A. \& Taher-Uz, Z. Human fetal hepatocyte transplantation in patients with fulminant hepatic failure. Transplantation 58, 951-952 (1994). 
340 Kaully, T., Kaufman-Francis, K., Lesman, A. \& Levenberg, S. Vascularization The Conduit to Viable Engineered Tissues. Tissue Eng Part B 15, 159-169 (2009).

341 Chiu, L. L., Iyer, R. K., Reis, L. A., Nunes, S. S. \& Radisic, M. Cardiac tissue engineering: current state and perspectives. Front Biosci 17, 1533-1550 (2012).

342 Jain, R. K. Molecular regulation of vessel maturations. Nat Med 9, 685-693 (2003).

343 Markkanen, J. E., Rissanen, T. T., Kivela, A. \& Yla-Herttuala, S. Growth factorinduced therapeutic angiogenesis and arteriogenesis in the heart--gene therapy. Cardiovasc Res 65, 656-664 (2005).

344 Han, J. et al. Adipose tissue is an extramedullary reservoir of functional hematopoetic stem and progenitor cells. Blood 115, 957-964, doi:10.1182/blood2009- (2010).

345 Hoying, J. B. \& Williams, S. K. Effects of basic fibroblast growth factor on human microvessel endothelial cell migration on collagen I correlates inversely with adhesion and is cell density dependent. J Cell Physiol 168, 294-304 (1996).

346 Chang, C. C. et al. Angiogenesis in a microvascular construct for transplantation depends on the method of chamber circulation. Tissue Eng Part A 16, 795-805 (2010).

347 Nunes, S. S., Rekapally, H., Chang, C. C. \& Hoying, J. B. Vessel arterial-venous plasticity in adult neovascularization phenotypes. Microvasc Res 79, 10-20 (2009). 
348 Boyd, N. L. et al. Microvascular mural cell functionality of human embryonic stem cell-derived mesenchymal cells. Tissue Eng Part A 17, 1537-1548, doi:10.1089/ten.TEA.2010.0397 (2011).

349 Carmona, R. et al. Immunolocalization of the transcription factor Slug in the developing avian heart. Anat Embryol (Berl) 201, 103-109 (2000).

350 Gao, Y., Hu, H. Z., Chen, K. \& Yang, J. Z. Primary porcine hepatocytes with portal vein serum cultured on microcarriers or in spheroidal aggregates. World $J$ Gastroenterol 6 (2000).

351 Nunes, S. S. et al. Angiogenic potential of microvessel fragments is independent of the tissue of origin and can be influenced by the cellular composition of the implants. Microcirculation 17, 557-567 (2010).

352 Nunes, S. S. et al. Implanted microvessels progress through distinct neovascularization phenotypes. Microvasc Res 79, 10-20, doi:10.1016/j.mvr.2009.10.001 (2010).

353 Langer, R. Tissue engineering: perspectives, challenges, and future directions. Tissue Eng Part A 13, 1-2 (2007).

354 Johnson, P. C., Mikos, A. G., Fisher, J. P. \& Jansen, J. A. Strategic directions in tissue engineering. Tissue Eng Part A 13, $2827-2837$ (2007).

$355 \mathrm{Au}, \mathrm{P}$. et al. Paradoxical effects of PDGF-BB overexpression in endothelial cells on engineered blood vessels in vivo. Am J Pathol 175, 294-302 (2009).

356 Folkman, J. \& Hochberg, M. Self-regulation of growth in three dimensions. J Exp Med 138, 745-753 (1973). 
357 Davis, G. E., Koh, W. \& Stratman, A. N. Mechanisms controlling human endothelial lumen formation and tube assembly in three-dimensional extracellular matrices. Birth Defects Res C Embryo Today 81, 270-285 (2007).

358 Gersh, I. \& Still, M. A. Blood vessels in fat tissue. Relation to problems of gas exchange. $J$ Exp Med 81, 219-232 (1945).

359 Pinheiro, C. H. et al. Local injections of adipose-derived mesenchymal stem cells modulate inflammation and increase angiogenesis ameliorating the dystrophic phenotype in dystrophin-deficient skeletal muscle. Stem Cell Rev 8, 363-374, doi:10.1007/s12015-011-9304-0 (2012).

360 Cai, J. et al. Directed differentiation of human embryonic stem cells into functional hepatic cells. Hepatology 45, 1229-1239, doi:10.1002/hep.21582 (2007).

361 Kedem, A. et al. Vascular endothelial growth factor-releasing scaffolds enhance vascularization and engraftment of hepatocytes transplanted on liver lobes. Tissue Eng Part A 11, 715-722 (2005).

362 Mizuno, H., Tobita, M. \& Uysal, A. C. Concise review: Adipose-derived stem cells as a novel tool for future regenerative medicine. Stem Cells 30, 804-810 (2012).

363 Broudy, V. C. et al. Human umbilical vein endothelial cells display high-affinity c-kit receptors and produce a soluble form of the c-kit receptor. Blood $\mathbf{8 3}, 2145$ 2152 (1994).

364 Lammie, A. et al. Expression of c-kit and kit ligand proteins in normal human tissues. J Histochem Cytochem 42, 1417-1425 (1994). 
365 Majumder, S., Brown, K., Qiu, F. H. \& Besmer, P. c-kit protein, a transmembrane kinase: identification in tissues and characterization. Mol Cell Biol 8, 4896-4903 (1988).

366 Abramsson, A., Lindblom, P. \& Betsholtz, C. Endothelial and nonendothelial sources of PDGF-B regulate pericyte recruitment and influence vascular pattern formation in tumors. J Clin Invest 112, 1142-1151 (2003).

367 Bjarnegard, M. et al. Endothelium-specific ablation of PDGFB leads to pericyte loss and glomerular, cardiac and placental abnormalities. Development 131, 18471857 (2004).

368 Zhao, Y., Waldman, S. D. \& Flynn, L. E. Multilineage co-culture of adiposederived stem cells for tissue engineering. J Tissue Eng Regen Med 9, 826-837 (2012).

369 Williams, S. K., Wang, T. F., Castrillo, R. \& Jarrell, B. E. Liposuction-derived human fat used for vascular graft sodding contains endothelial cells and not mesothelial cells as the major cell type. J Vasc Surg 19, 916-923 (1994).

370 Maijub, J. G. et al. Concentration Dependent Vascularization of Adipose Stromal Vascular Fraction Cells. Cell Transplant, doi:10.3727/096368914X685401 (2014).

371 Madri, J. A. \& Williams, S. K. Capillary endothelial cell cultures: phenotypic modulation by matrix components. Journal of Cell Biology 97, 153-165 (1983).

372 Madri, J. A., Pratt, B. M. \& Tucker, A. M. Phenotypic modulation of endothelial cells by transforming growth factor-beta depends upon the composition and organization of the extracellular matrix. J.Cell Biol. 106, 1375-1384 (1988). 
373 Wiley, D. M. et al. Distinct signalling pathways regulate sprouting angiogenesis from the dorsal aorta and the axial vein. Nat Cell Biol 13, 686-692, doi:10.1038/ncb2232 (2011).

374 Ladwein, M. \& Rottner, K. On the Rho'd: the regulation of membrane protrusions by Rho-GTPases. FEBS Lett 582, 2066-2074, doi:10.1016/j.febslet.2008.04.033 (2008).

375 Hasan, S. S. \& Siekmann, A. F. The same but different: signaling pathways in control of endothelial cell migration. Curr Opin Cell Biol 36, 86-92, doi:10.1016/j.ceb.2015.07.009 (2015).

376 Chen, B. et al. Small molecule-mediated disruption of Wnt-dependent signaling in tissue regeneration and cancer. Nat Chem Biol 5, 100-107, doi:10.1038/nchembio.137 (2009).

377 Murdoch, B. et al. Wnt-5A augments repopulating capacity and primitive hematopoietic development of human blood stem cells in vivo. Proc Natl Acad Sci U S A 100, 3422-3427, doi:10.1073/pnas.0130233100 (2003).

378 Blumenthal, A. et al. The Wingless homolog WNT5A and its receptor Frizzled-5 regulate inflammatory responses of human mononuclear cells induced by microbial stimulation. Blood 108, 965-973, doi:10.1182/blood-2005-12-5046 (2006).

379 Cheng, C. W., Yeh, J. C., Fan, T. P., Smith, S. K. \& Charnock-Jones, D. S. Wnt5a-mediated non-canonical Wnt signalling regulates human endothelial cell proliferation and migration. Biochem Biophys Res Commun 365, 285-290, doi:10.1016/j.bbrc.2007.10.166 (2008). 
380 Lin, L. \& Sherman, P. D. Cleansing Data the Chauvinet Way. SESUG Proceedings, 1-11 (2007).

381 Montesano, R., Orci, L. \& Vassalli, P. In vitro rapid organization of endothelial cells into capillary-like networks is promoted by collagen matrices. J Cell Biol 97, 1648-1652 (1983).

382 Willert, K. et al. Wnt proteins are lipid-modified and can act as stem cell growth factors. Nature 423, 448-452 (2003).

383 Reis, M. et al. Endothelial Wnt/beta-catenin signaling inhibits glioma angiogenesis and normalizes tumor blood vessels by inducing PDGF-B expression. $J$ Exp Med 209, 1611-1627, doi:10.1084/jem.20111580 (2012).

384 Gherghe, C. M. et al. Wnt1 is a proangiogenic molecule, enhances human endothelial progenitor function, and increases blood flow to ischemic limbs in a HGF-dependent manner. FASEB $J$ 25, 1836-1843, doi:10.1096/fj.10-172981 (2011).

385 Klein, D. et al. Wnt2 acts as an angiogenic growth factor for non-sinusoidal endothelial cells and inhibits expression of stanniocalcin-1. Angiogenesis 12, 251265, doi:10.1007/s10456-009-9145-5 (2009).

386 Daneman, R. et al. Wnt/B-catenin signaling is required for CNS, but not nonCNS, angiogenesis. Proceedings of the National Academy of Sciences 106, 641646, doi:10.1073/pnas.0901563106 (2009).

387 Jover, B., Girardot, D., de Courtois Roy de Vacquieres, F., Casellas, D. \& Moles, J. P. Wnt-4 potently inhibits capillary outgrowth from rat aorta in 3D culture. 
Fundam Clin Pharmacol 27, 465-470, doi:10.1111/j.1472-8206.2012.01044.x (2013).

388 Arderiu, G., Espinosa, S., Peña, E., Aledo, R. \& Badimon, L. Monocyte-secreted Wnt5a interacts with FZD5 in microvascular endothelial cells and induces angiogenesis through tissue factor signaling. J Mol Cell Biol 6, 380-393, doi:10.1093/jmcb/mju036 (2014).

389 Murdoch, C. E., Bachschmid, M. M. \& Matsui, R. Regulation of neovascularization by S-glutathionylation via the Wnt5a/sFlt-1 pathway. Biochem Soc Trans 42, 1665-1670, doi:10.1042/BST20140213 (2014).

390 Melgar-Lesmes, P. \& Edelman, E. R. Monocyte-endothelial cell interactions in the regulation of vascular sprouting and liver regeneration in mouse. J Hepatol, doi:10.1016/j.jhep.2015.05.011 (2015).

391 Ekström, E. J. et al. WNT5A induces release of exosomes containing proangiogenic and immunosuppressive factors from malignant melanoma cells. Molecular Cancer 13, 1-15 (2014).

392 Korn, C. et al. Endothelial cell-derived non-canonical Wnt ligands control vascular pruning in angiogenesis. Development 141, 1757-1766, doi:10.1242/dev.104422 (2014).

393 Bian, W. J. et al. A novel Wnt5a-Frizzled4 signaling pathway mediates activityindependent dendrite morphogenesis via the distal PDZ motif of Frizzled 4. Dev Neurobiol 75, 805-822, doi:10.1002/dneu.22250 (2015). 
394 Sen, M., Chamorro, M., Reifert, J., Corr, M. \& Carson, D. A. Blockade of Wnt5A/frizzled 5 signaling inhibits rheumatoid synoviocyte activation. Arthritis Rheum 44, $772-781$ (2001).

395 Oishi, I. et al. The receptor tyrosine kinase Ror2 is involved in non-canonical Wnt5a/JNK signalling pathway. Genes to Cells 8, 645-654 (2003).

396 Liu, Y., Rubin, B., Bodine, P. V. \& Billiard, J. Wnt5a induces homodimerization and activation of Ror2 receptor tyrosine kinase. J Cell Biochem 105, 497-502, doi:10.1002/jcb.21848 (2008).

397 Angers, S. \& Moon, R. T. Proximal events in Wnt signal transduction. Nat Rev Mol Cell Biol 10, 468-477, doi:10.1038/nrm2717 (2009).

398 Catalan, V. et al. Activation of noncanonical Wnt signaling through WNT5A in visceral adipose tissue of obese subjects is related to inflammation. $J$ Clin Endocrinol Metab 99, E1407-1417, doi:10.1210/jc.2014-1191 (2014).

399 Maschauchán, T. N. H. et al. Wnt5a signaling induces proliferation and survival of endothelial cells in vitro and expression of MMP-1 and Tie-2. Molecular Biology of the Cell 17, 5163-5172, doi:10.1091/mbc.E06 (2006).

400 Morris, M. E. et al. Systemically delivered adipose stromal vascular fraction cells disseminate to peripheral artery walls and reduce vasomotor tone through a CD11b+ cell-dependent mechanism. Stem Cells Transl Med 4, 369-380, doi:10.5966/sctm.2014-0252 (2015).

401 Zuk, P. A. et al. Multilineage cells from human adipose tissue: implications for cell-based therapies. Tissue Eng 7, 211-228 (2001). 
402 Lin, G. et al. Defining stem and progenitor cells within adipose tissue. Stem Cells Dev 17, 1053-1063, doi:10.1089/scd.2008.0117 (2008).

403 Butler, M. J. \& Sefton, M. V. Cotransplantation of adipose-derived mesenchymal stromal cells and endothelial cells in a modular construct drives vascularization in SCID/bg mice. Tissue Eng Part A 18, 1628-1641, doi:10.1089/ten.TEA.2011.0467 (2012).

404 Frerich, B., Zuckmantel, K. \& Hemprich, A. Microvascular engineering in perfusion culture: immunohistochemistry and CLSM findings. Head Face Med 2 , 26, doi:10.1186/1746-160X-2-26 (2006).

405 Olson, L. E. \& Soriano, P. PDGFRbeta signaling regulates mural cell plasticity and inhibits fat development. Dev Cell 20, 815-826, doi:10.1016/j.devcel.2011.04.019 (2011).

406 Tallquist, M. D., Soriano, P. \& Klinghoffer, R. A. Growth factor signaling pathways in vascular development. Oncogene 18, 7917-7932 (1999).

407 Lindahl, P., Johansson, B. R., Levéen, P. \& Betsholtz, C. Pericyte Loss and Microaneurysm Formation in PDGF-B-Deficient Mice. Science 277, 242-245 (1997).

408 Lindblom, P. et al. Endothelial PDGF-B retention is required for proper investment of pericytes in the microvessel wall. Genes \& Dev 17, 1835-1840, doi:10.1101/ (2003).

409 Hirschi, K. K., Robovsky, S. A. \& D'Amore, P. A. PDGF, TGF-beta, and Heterotypic Cell-Cell Interactions Mediate Endothelial Cell-induced Recruitment 
of 10T1/2 Cells and Their Differentiation to a Smooth Muscle Fate. J Cell Biol 141, 805-814 (1998).

410 Goumans, M. J. et al. Balancing the activation state of the endothelium via two distinct TGF-beta type I receptors. The EMBO Journal 21, 1743-1753 (2002).

411 Lebrin, F. et al. Endoglin promotes endothelial cell proliferation and TGFbeta/ALK1 signal transduction. The EMBO Journal 23, 4018-4028, doi:10.1038/ (2004).

412 Dejana, E. The role of wnt signaling in physiological and pathological angiogenesis. Circ Res 107, 943-952, doi:10.1161/CIRCRESAHA.110.223750 (2010).

413 Goodwin, A. M., Sullivan, K. M. \& D'Amore, P. A. Cultured endothelial cells display endogenous activation of the canonical Wnt signaling pathway and express multiple ligands, receptors, and secreted modulators of Wnt signaling. Dev Dyn 235, 3110-3120, doi:10.1002/dvdy.20939 (2006).

414 Newman, A. C. \& Hughes, C. C. W. Macrophages and angiogenesis: a role for Wnt signaling. Vascular Cell 4, 1-7 (2012).

415 Parish, C. L. et al. Wnt5a-treated midbrain neural stem cells improve dopamine cell replacement therapy in parkinsonian mice. J Clin Invest 118, 149-160, doi:10.1172/JCI32273 (2008).

416 Potok, M. A. et al. WNT signaling affects gene expression in the ventral diencephalon and pituitary gland growth. Dev Dyn 237, 1006-1020, doi:10.1002/dvdy.21511 (2008). 
417 Himmelreich, N., Kaufmann, L. T., Steinbeisser, H., Korner, C. \& Thiel, C. Lack of phosphomannomutase 2 affects Xenopus laevis morphogenesis and the noncanonical Wnt5a/Ror2 signalling. $J$ Inherit Metab Dis 38, 1137-1146, doi:10.1007/s10545-015-9874-0 (2015).

418 Takahashi, K. \& Yamanaka, S. Induction of pluripotent stem cells from mouse embryonic and adult fibroblast cultures by defined factors. Cell. 126, 663-676, doi:10.1016/j.cell.2006.07.024 (2006).

419 Rader, D. J., Cohen, J. \& Hobbs, H. H. Monogenic hypercholesterolemia: new insights in pathogenesis and treatment. J Clin Invest. 111, 1795-1803, doi:10.1172/jci200318925 (2003).

420 Benn, M., Watts, G. F., Tybjaerg-Hansen, A. \& Nordestgaard, B. G. Familial hypercholesterolemia in the danish general population: prevalence, coronary artery disease, and cholesterol-lowering medication. J Clin Endocrinol Metab. 97, 3956-3964, doi:10.1210/jc.2012-1563 (2012).

421 Nordestgaard, B. G. et al. Familial hypercholesterolaemia is underdiagnosed and undertreated in the general population: guidance for clinicians to prevent coronary heart disease: consensus statement of the European Atherosclerosis Society. Eur Heart J. 34, 3478-3490a, doi:10.1093/eurheartj/eht273 (2013).

422 Kim, W. R. et al. OPTN/SRTR 2012 Annual Data Report: liver. Am J Transplant. 14, 69-96, doi:10.1111/ajt.12581 (2014).

423 Treyer, A. \& Müsch, A. Hepatocyte polarity. Compr Physiol. 3, 243-287, doi:10.1002/cphy.c120009. (2013). 
424 Hibbitt, O. C. et al. Long-term physiologically regulated expression of the lowdensity lipoprotein receptor in vivo using genomic DNA mini-gene constructs. Mol Ther. 18, 317-326, doi:10.1038/mt.2009.249 (2010).

425 Boyd, N. L., Robbins, K. R., Dhara, S. K., West, F. D. \& Stice, S. L. Human Embryonic Stem Cell-Derived Mesoderm-Like Epithelium Transitions to Mesenchymal Progenitor Cells. Tissue Eng Part A. 15, 1897-1907, doi:10.1089/ten.tea.2008.0351 (2009).

426 Mitalipova, M. M. et al. Preserving the genetic integrity of human embryonic stem cells. Nat Biotechnol. 23, 19-20, doi:10.1038/nbt0105-19 (2005).

427 Hentze, H. et al. Teratoma formation by human embryonic stem cells: evaluation of essential parameters for future safety studies. Stem Cell Res. 2, 198-210, doi:10.1016/j.scr.2009.02.002 (2009).

428 Song, Z. et al. Efficient generation of hepatocyte-like cells from human induced pluripotent stem cells. Cell Res. 19, 1233-1242, doi:10.1038/cr.2009.107 (2009).

429 Lufino, M. M., Manservigi, R. \& Wade-Martins, R. An S/MAR-based infectious episomal genomic DNA expression vector provides long-term regulated functional complementation of LDLR deficiency. Nucleic Acids Res. 35, e98, doi:10.1093/nar/gkm570 (2007).

430 Goldstein, J. L. \& Brown, M. S. Binding and Degradation of Low Density Lipoproteins by Cultured Human Fibroblasts. J Biol Chem. 249, 5153-5162 (1974). 
431 Warren, L. et al. Highly efficient reprogramming to pluripotency and directed differentiation of human cells with synthetic modified mRNA. Cell Stem Cell. 7, 618-630, doi:10.1016/j.stem.2010.08.012 (2010).

432 Takahashi, K. et al. Induction of pluripotent stem cells from adult human fibroblasts by defined factors. Cell. 131, 861-872, doi:10.1016/j.cell.2007.11.019 (2007).

433 Boyer, L. A. et al. Core transcriptional regulatory circuitry in human embryonic stem cells. Cell. 122, 947-956, doi:10.1016/j.cell.2005.08.020 (2005).

434 Fattahi, F. et al. Disease-corrected hepatocyte-like cells from familial hypercholesterolemia-induced pluripotent stem cells. Mol Biotechnol. 54, 863873, doi:10.1007/s12033-012-9635-3 (2013).

435 Kakaei, F. et al. Liver transplantation for homozygous familial hypercholesterolemia: two case reports. Transplant Proc. 41, 2939-2941, doi:10.1016/j.transproceed.2009.07.028 (2009).

436 Wiegand, B. D., Ketterer, S. G. \& Rapaport, E. The use of indocyanine green for the evaluation of hepatic function and blood flow in man. Am J Dig Dis. 5, 427436 (1960).

437 Maijub, J. G. et al. Concentration Dependent Vascularization of Adipose Stromal Vascular Fraction Cells. Cell Transplant. In press, doi:10.3727/096368914X685401 (2014).

438 Nunes, S. S. et al. Generation of a functional liver tissue mimic using adipose stromal vascular fraction cell-derived vasculatures. Sci Rep. 3, 2141, doi:10.1038/srep02141 (2013). 
439 Krieger, M., Brown, M. S. \& Goldstein, J. L. Isolation of Chinese Hamster Cell Mutants Defective in the Receptor-mediated Endocytosis of Low Density Lipoprotein. J Mol Biol. 150, 167-184 (1981).

440 Ma, P. T. S. et al. Mevinolin, an inhibitor of cholesterol synthesis, induces mRNA for low density lipoprotein receptor in livers of hamsters and rabbits. Proc Natl Acad Sci USA. 83, 8370-8374 (1986).

441 Ho, Y. K., Brown, M. S., Bilheimer, D. W. \& Goldstein, J. L. Regulation of Low Density Lipoprotein Receptor Activity in Freshly Isolated Human Lymphocytes. $J$ Clin Invest. 58, 1465-1474 (1976).

442 Grossman, M. et al. A pilot study of ex vivo gene therapy for homozygous familial hypecholesterolemia. Nat Med. 1, 1148-1154, doi:10.1038/nm1195-1148 (1995).

443 Grossman, M. et al. Successful ex vivo gene therapy directed to liver in a patient with familial hypercholesterolemia. Nat Genet. 6, 335-341, doi:10.1038/ng0494335 (1994).

444 Cichon, G. et al. Non-physiological overexpression of the low density lipoprotein receptor (LDLr) gene in the liver induces pathological intracellular lipid and cholesterol storage. J Gene Med. 6, 166-175, doi:10.1002/jgm.473 (2004).

445 Heeren, J., Weber, W. \& Beisiegel, U. Intracellular processing of endocytosed triglyceride-rich lipoproteins comprises both recycling and degradation. $J$ Cell Sci. 112, 349-359 (1999). 
446 Leight, E. R. \& Sugden, B. The cis-acting family of repeats can inhibit as well as stimulate establishment of an oriP replicon. J Virol. 75, 10709-10720, doi:10.1128/JVI.75.22.10709-10720.2001 (2001).

447 Leight, E. R. \& Sugden, B. Establishment of an oriP replicon is dependent upon an infrequent, epigenetic event. Mol Cell Biol. 21, 4149-4161, doi:10.1128/MCB.21.13.4149-4161.2001 (2001).

448 Costa, M. et al. A method for genetic modification of human embryonic stem cells using electroporation. Nat Protoc. 2, 792-796, doi:10.1038/nprot.2007.105 (2007).

449 Maxfield, F. R. \& Menon, A. K. Intracellular sterol transport and distribution. Curr Opin Cell Biol. 18, 379-385, doi:10.1016/j.ceb.2006.06.012 (2006).

450 Rachid, S. C., S; Hannan, N; Marcinak, SJ; Miranda, E; Alexander, G; HuangDoran, I; Griffin, J; Ahrlund-Richter, L; Skepper, J; Semple, R; Weber, A; Lomas, DA; Valler, L. Modeling inherited metabolic disorders of the liver using human induced pluripotent stem cells. JCI 120, 3127-3136, doi:10.1172/jci43122ds1 (2010).

451 Jacobs, F., Van Craeyveld, E., Feng, Y., Snoeys, J. \& De Geest, B. Adenoviral low density lipoprotein receptor attenuates progression of atherosclerosis and decreases tissue cholesterol levels in a murine model of familial hypercholesterolemia. Atherosclerosis 201, 289-297 (2008).

452 Kassim, S. H. et al. Gene therapy in a humanized mouse model of familial hypercholesterolemia leads to marked regression of atherosclerosis. PLoS One $\mathbf{5}$ (2010). 
453 Stein, C. S., Martins, I. \& Davidson, B. L. Long-term reversal of hypercholesterolemia in low density lipoprotein receptor (LDLR)-deficient mice by adenovirus-mediated LDLR gene transfer combined with CD154 blockade. $J$ Gene Med 2, 41-51 (2000).

$454 \mathrm{Li}, \mathrm{C}$. Y. et al. Micropatterned cell-cell interactions enable functional encapsulation of primary hepatocytes in hydrogel microtissues. Tissue Eng Part A 20, 2200-2212 (2014).

455 Löser, P., Jennings, G. S., Strauss, M. \& Sandig, V. Reactivation of the Previously Silenced Cytomegalovirus Major Immediate-Early Promoter in the Mouse Live: Involvement of NFkB. J Virol 72, 180-190 (1998).

456 Thomas, C. E., Ehrhardt, A. \& Kay, M. A. Progress and problems with the use of viral vectors for gene therapy. Nat Rev Genet 4, 346-358 (2003).

457 Kozarsky, K. F. et al. In vivo correction of low density lipoprotein receptor deficiency in the Watanabe heritable hyperlipidemic rabbit with recombinant adenoviruses. J Biol Chem 269, 13695-13702 (1994).

458 Van Craeyveld, E., Jacobs, F., Gordts, S. C. \& De Geest, B. Gene therapy for Familial Hypercholesterolemia. Curr Pharm Des 17, 2575-2591 (2011).

459 Costa, M. et al. A method for genetic modification of human embryonic stem cells using electroporation. Nat Protoc 2, 792-796, doi:10.1038/nprot.2007.105 (2007).

460 Yates, J. L. \& Guan, N. Epstein-Barr virus-derived plasmids replicate only once per cell cycle and are not amplified after entry into cells. $J$ Virol. $\mathbf{6 5}, 483-488$ (1991). 
461 Levitskaya, J., Sharipo, A., Leonchiks, A., Ciechanover, A. \& Masucci, M. G. Inhibition of ubiquitin/proteasome-dependent protein degradation by the Gly-Ala repeat domain of the Epstein-Barr virus nuclear antigen 1. Proc Natl Acad Sci U S A 94, 12616-12621 (1997).

462 Goldstein, J. L. \& Brown, M. S. The LDL receptor. Arterioscler Thromb Vasc Biol 29, 431-438, doi:10.1161/ATVBAHA.108.179564 (2009).

463 Song, H., Chung, S. K. \& Xu, Y. Modeling disease in human ESCs using an efficient BAC-based homologous recombination system. Cell Stem Cell 6, 80-89, doi:10.1016/j.stem.2009.11.016 (2010).

464 Irion, S. et al. Identification and targeting of the ROSA26 locus in human embryonic stem cells. Nat Biotechnol 25, 1477-1482, doi:10.1038/nbt1362 (2007).

465 Mohr, J. C., de Pablo, J. J. \& Palacek, S. P. Electroporation of Human Embryonic Stem Cells: Small and Macromolecule Loading and DNA Transfection. Biotechnol Prog 22, 825-834 (2006).

466 Leight, E. R. \& Sugden, B. Establishment of an oriP replicon is dependent upon an infrequent, epigenetic event. Mol Cell Biol 21, 4149-4161, doi:10.1128/MCB.21.13.4149-4161.2001 (2001).

$467 \mathrm{Li}$, J. et al. In vivo gene therapy for hyperlipidemia: phenotypic correction in Watanabe rabbits by hepatic delivery of the rabbit LDL receptor gene. $J$ Clin Invest. 95, 768-773, doi:10.1172/JCI117725 (1995).

468 Brown, M. S., Goldstein, J. L., Havel, R. J. \& Steinberg, D. Gene therapy for cholesterol. Nature genetics 7, 349-350 (1994). 
469 Hobbs, H. H., Brown, M. S. \& Goldstein, J. L. Molecular Genetics of the LDL Receptor Gene in Familial Hypercholesterolemia. Human Mutation 1, 445-466 (1992).

470 Hunter, D. D., Zhang, M., Ferguson, J. W., Koch, M. \& Brunken, W. J. The extracellular matrix component WIF-1 is expressed during, and can modulate, retinal development. Mol Cell Neurosci 27, 477-488 (2004).

471 Shen, G. et al. Structural basis of the Norrin-Frizzled 4 interaction. Cell Res $\mathbf{2 5}$ (2015).

472 Umbhauer, M. et al. The C-terminal cytoplasmic Lys-thr-X-X-X-Trp motif in frizzled receptors mediates Wnt/beta-catenin signalling. EMBO J 19, 4944-4954 (2000).

473 Sheldahl, L. C., Park, M., Malbon, C. C. \& Moon, R. T. Protein kinase C is differentially stimulated by Wnt and Frizzled homologs in a G-protein-dependent manner. Curr Biol. 9, 695-698 (1999). 


\section{CURRICULUM VITA}

NAME:

ADDRESS:

DOB:

EDUCATION

\& TRAINING:
Venkat Madhav Ramakrishnan

Cardiovascular Innovation Institute

University of Louisville School of Medicine

302 E. Muhammad Ali Boulevard

Suite 512/513

Louisville, KY 40202

Lexington, Kentucky - February 17, 1988

M.D.

University of Louisville School of Medicine

2011-present

Ph.D., Physiology \& Biophysics

University of Louisville School of Medicine

2013-2015

M.S., Physiology \& Biophysics

University of Louisville School of Medicine 2013-2014

Fulbright Scholar, Urologic Tissue Engineering University Hospital Zürich

2010-2011

B.S., Biology - Cellular / Physiology

University of Louisville

2006-2010

Michael Tanner Memorial Award for Excellence in Graduate Student Sciences

2014 
Norton Healthcare Medical Student Research Award, $2^{\text {nd }}$ Place 2013

Magna cum Laude

2010

University Honors Scholar

2010

Certificate of Honors, Department of Biology

2010

Cardinal Award of Excellence - Scholar/Leader

2010

Honors Thesis Presentation: "Identification of Single Nucleotide Polymorphisms Associated with Metastatic Prostate Cancer" 2010

Arts \& Sciences Alumni Council Overall Achievement Award 2009

Dean's List and Dean's Scholar

2007-2010

Guaranteed Entrance to Medical School (GEMS)

2006-2010

Kentucky Governor's Scholar

2005

SCHOLARSHIPS

\& FELLOWSHIPS: Integrated Programs in the Biomedical Sciences (IPIBS) Graduate Fellowship

2013-2015

Summer Research Scholars Program (SRSP)

2012, 2013

Physician-Scientist Training Program (M.D. / Ph.D.) Scholarship 2011-present

Summer Urological Research Experience (SURE) Program at the Johns Hopkins Brady Urological Institute

2011 
J. William Fulbright Research Fellowship, Switzerland 2010-2011

Alvin J. Siteman Cancer Center Summer Opportunities Program at Washington University School of Medicine in St. Louis 2010

National Merit Finalist Scholarship

2006-2010

Grawemeyer Scholarship

2006-2010

PROFESSIONAL SOCIETIES:

International Society for Stem Cell Research 2014-2015

American Heart Association

2013-2015

American Physician Scientists Association

2013-present

Tissue Engineering \& Regenerative Medicine International Society 2013-present

Greater Louisville Medical Society

2011-present

\section{PUBLICATIONS:}

1. Kiehl N, Kinsey S, Ramakrishnan V, DaJusta DG. "Pediatric Prostatic Abscess." Urology (Gold Journal). 2012 Dec; 80(6): pp. 1364-5.

2. Boyd NL, Nunes SS, Krishnan L, Jokinen JD, Ramakrishnan VM, Bugg AR, Hoying JB. "Dissecting the role of human embryonic stem cellderived mesenchymal cells in human umbilical vein endothelial cell network stabilization in three-dimensional environments." Tissue Engineering Part A. 2013 Jan; 19(1-2): pp. 211-23.

3. Nunes SS, Maijub JG, Krishnan L, Ramakrishnan VM, Clayton LR, Williams SK, Hoying JB*, Boyd NL*. "Generation of a functional liver tissue mimic using adipose stromal vascular fraction cell-derived vasculatures". Scientific Reports. 2013 Jul; (3): pp. 1-7. 
4. Largo RA*, Ramakrishnan VM*, Marschall JS*, Ziogas A, Eberli D**, Ehrbar M**. "Long-term Biostability and Bioactivity of Engineered VEGF $_{121}$ in vitro and in vivo". Biomaterials Science. 2014 Apr; (2): pp. 581-590.

5. Ramakrishnan VM*, Yang JY*, Tien KT, McKinley TR, Bocard BR, Maijub JG, Burchell PO, Williams SK, Morris, ME, Hoying, JB, WadeMartins R, West FD, Boyd NL. "Restoration of Physiologically Responsive Low-Density Lipoprotein Receptor-Mediated Endocytosis in Genetically Deficient Induced Pluripotent Stem Cells”. Scientific Reports. 2015 Aug (in press).

6. Ramakrishnan VM, Tien KT, McKinley TR, Bocard BR, McCurry TM, Williams SK, Hoying JB, Boyd NL. "Wnt5a Modulates the Assembly of Human Stromal Vascular Fraction-Derived Microvasculatures" (submitted).

\section{BOOK CHAPTER:}

1. R.A. Largo*, V.M. Ramakrishnan*, M. Ehrbar, A. Ziogas, J. Plock, and D. Eberli. "Angiogenesis and Vascularity for Tissue Engineering Applications." Regenerative Medicine and Tissue Engineering: From Cells to Organs (Book 1). ISBN: 978-953-307-337-8. InTech Publishing Group.

\section{EDITORIAL:}

1. "Modern Technology and the Changing Medical Landscape" V.M. Ramakrishnan. Louisville Medicine (Greater Louisville Medical Society). Volume 63, Issue 5, October 2015.

\section{ABSTRACT ORAL \& POSTER PRESENTATIONS:}

1. "Effect of Carbon Nano Tubes on the Compression and Flexural Properties of Calcium Sulfate," V.M. Ramakrishnan, R. Andrews, and D.A. Puleo, Annual Meeting and Exhibition of the American and Canadian Associations for Dental Research (AADR), Orlando, FL. $(03 / 2006)$

2. "Effects of Hydrogel Nanoparticles and Protein-Adsorbed Calcium Sulfate Particles on Mechanical Properties of Calcium Sulfate," V.M. Ramakrishnan, B. Orellana, M.V. Thomas, J.Z. Hilt, and D.A. Puleo, Society for Biomaterials' 2009 Annual Meeting, San Antonio, TX. (04/2009)

3. "Fibrin TG-VEGF is a Supporter of Early Angiogenesis in Urinary Sphincter Engineering," R.A. Largo*, V.M. Ramakrishnan*, A. Ziogas, 
M. Ehrbar, J. Plock, and D. Eberli, Schweizerischen Gesellschaft für Urologie (SGU; Swiss Congress of Urology) $67^{\text {th }}$ Annual Meeting, Basel, Switzerland. (09/2011).

4. "Fibrin TG-VEGF is a Supporter of Early Angiogenesis in Urinary Sphincter Engineering," R.A. Largo*, V.M. Ramakrishnan*, A. Ziogas, M. Ehrbar, J. Plock, and D. Eberli, European Association of Urology (EAU) 2012 Annual Meeting, Paris, France. (02/2012).

5. "Rare Germline Variants Associate with Aggressive Forms of Prostate Cancer," L.A. Tessler, V.M. Ramakrishnan, B. Wu, R. Goldfeder, R.D. Mitra, and A.S. Kibel, American Urological Association (AUA) 2012 Annual Meeting, Atlanta, GA (05/2012). ORAL PRESENTATION

6. "Fibrin TG-VEGF is a Supporter of Early Angiogenesis in Urinary Tissue Engineering," R.A. Largo*, V.M. Ramakrishnan*, A. Ziogas, M. Ehrbar, J. Plock, and D. Eberli, AUA 2012 Annual Meeting, Atlanta, GA (5/2012). ORAL PRESENTATION

7. "Long-term Cell Demanded Release of TG-VEGF 121 for the Development of Mature Vascular Networks In Vivo," R.A. Largo*, J.S. Marschall*, A. Banfi, V.M. Ramakrishnan, A. Ziogas, T. Sulser, K. Lorentz, J. Hubbell, M. Ehrbar, and D. Eberli, Tissue Engineering and Regenerative Medicine World Congress (TERMIS) 2012 Annual Meeting, Vienna, Austria (09/2012). ORAL PRESENTATION

8. "Pre-establishment of a Vascular Network for Urinary Sphincter Engineering," R.A. Largo, V.M. Ramakrishnan*, J.S. Marschall*, A. Ziogas, J. Plock, M. Ehrbar, and D. Eberli, TERMIS 2012 World Congress Meeting, Vienna, Austria (09/2012).

9. "Development of an engineered implantable vascularized cell-based LDL apheresis device," V.M. Ramakrishnan, J.G. Maijub, S.K. Williams, N.L. Boyd, $1^{\text {st }}$ Annual South-Central MD-PhD Conference, University of Louisville, Louisville, KY (09/2012).

10. "Development of an engineered implantable vascularized cell-based LDL apheresis device," V.M. Ramakrishnan, J.G. Maijub, M.E. Morris, J.B. Hoying, S.K. Williams, N.L. Boyd, Research!Louisville 2012 Annual Meeting, University of Louisville, Louisville, KY (09/2012).

11. "Development of an engineered implantable vascularized cell-based LDL apheresis device," J.G. Maijub, V.M. Ramakrishnan, S.S. Nunes, J. Dale, A.R. Bugg, M.E. Morris, J.B. Hoying, S.K. Williams, N.L. Boyd, Biomedical Engineering Society (BMES) 2012 Annual Meeting, Atlanta, GA (10/2012). 
12. "Vascularizing the Engineered Tissue," N.L. Boyd, S.S. Nunes, J.G. Maijub, V.M. Ramakrishnan, J.B. Hoying, and S.K. Williams, Materials Research Society (MRS) 2013 Annual Meeting, San Francisco, CA (04/2013).

13. "Adipose Stromal Vascular Fraction-Derived Vasculature Supports Implantation of Parenchymal Hepatocytes," V.M. Ramakrishnan*, P.O. Burchell*, J.I. Beier, J.R. Dale, G.E. Arteel, J.B. Hoying, S.K. Williams, and N.L. Boyd. Research!Louisville 2013 Annual Meeting, University of Louisville, Louisville, KY (09/2013).

14. "Adipose Stromal Vascular Fraction-Derived Vasculature Supports Implantation of Parenchymal Hepatocytes," V.M. Ramakrishnan*, P.O. Burchell*, J.I. Beier, J.R. Dale, G.E. Arteel, J.B. Hoying, S.K. Williams, and N.L. Boyd. American Physician Scientists Association (APSA) 2013 Midwestern Meeting, The Ohio State University, Columbus, OH $(11 / 2013)$.

15. "Characterization of Familial Hypercholesterolemia Patient-Derived Hepatocyte-Like Cells,” K.T. Tien, V.M. Ramakrishnan, and N.L. Boyd. DuPont Manual Regional Science and Engineering Fair, University of Louisville, Louisville, KY (03/2014)

16. "Characterization of Familial Hypercholesterolemia Patient-Derived Hepatocyte-Like Cells,” K.T. Tien, V.M. Ramakrishnan, and N.L. Boyd. Kentucky Junior Academy of Science, University of Kentucky, Lexington, KY $(06 / 2014)$

17. "Episomal Plasmid Functionally Corrects Monogenic-Deficient Induced Pluripotent Stem Cells," V.M. Ramakrishnan, J.Y. Yang, P.O. Burchell, K.T. Tien, B.R. Bocard, J.G. Maijub, S.K. Williams, J.B. Hoying, F.D. West, and N.L. Boyd. Research!Louisville 2014 Annual Meeting, University of Louisville, Louisville, KY (09/2014)

18. "Generation and LDLR Functional Correction of Familial Hypercholesterolemia iPSC with Physiologically Responsive NonIntegrating BAC Vector," V.M. Ramakrishnan, J.Y. Yang, P.O. Burchell, K.T. Tien, B.R. Bocard, J.G. Maijub, S.K. Williams, R. WadeMartins, F.D. West, and N.L. Boyd. American Heart Association (AHA) Scientific Sessions 2014 Annual Meeting, Chicago, IL (11/2014); published in the supplement to Circulation, Volume 130, 2014, Abstract A15669.

19. "Characterization of the Spontaneous Capillary-Like Network Formation of Human Stromal Vascular Fraction Cells," B.R. Bocard, K.T. Tien, 
V.M. Ramakrishnan, and N.L. Boyd. Kentucky Academy of Sciences Centennial Meeting, Lexington, KY (11/2014).

20. "Characterization of Familial Hypercholesterolemia Patient-Derived Hepatocyte-Like Cells," K.T. Tien, V.M. Ramakrishnan, and N.L. Boyd. American Junior Academy of Science: AAS Conference, San José, CA $(02 / 2015)$

21. "Characterization of the Spontaneous Capillary-Like Network Formation of Human Stromal Vascular Fraction Cells," K.T. Tien, B.R. Bocard, V.M. Ramakrishnan, and N.L. Boyd. Kentucky Junior Science and Humanities Symposium, University of Louisville, Louisville, KY $(02 / 2015)$

22. "Characterization of the Spontaneous Capillary-Like Network Formation of Human Stromal Vascular Fraction Cells," K.T. Tien, B.R. Bocard, V.M. Ramakrishnan, and N.L. Boyd. DuPont Manual Regional Science and Engineering Fair, University of Louisville, Louisville, KY (03/2015)

23. "Characterization of the Spontaneous Capillary-Like Network Formation of Human Stromal Vascular Fraction Cells," K.T. Tien, B.R. Bocard, V.M. Ramakrishnan, and N.L. Boyd. Kentucky Science and Engineering Fair, Eastern Kentucky University, Richmond, KY (03/2015)

24. "Wnt5a Modulates In Vitro Spontaneous Endothelial Networking in Human Adipose-Derived Stromal Vascular Fraction Cells," V.M. Ramakrishnan, K.T. Tien, B.R. Bocard, S.K. Williams, J.B. Hoying, and N.L. Boyd. $1^{\text {st }}$ Annual UofL Vascular Research Symposium, University of Louisville, Louisville, KY (04/2015)

25. "Wnt5a Modulates Early Angiogenesis of Human Stromal Vascular Fraction Cells In Vitro," V.M. Ramakrishnan. $30^{\text {th }}$ Annual $\mathrm{MD} / \mathrm{PhD}$ National Student Conference, Keystone, CO (07/2015)

26. "Generation of an Engineered, Vascularized, iPSC-Derived Hepatic Module," V.M. Ramakrishnan, J.Y. Yang, K.T. Tien, J.G. Maijub, P.O. Burchell, B.R. Bocard, S.K. Williams, M.E. Morris, J.B. Hoying, R. Wade-Martins, F.D. West, and N.L. Boyd. TERMIS 2015 4th World Congress Meeting, Boston, MA (09/2015); published in Tissue Engineering: Part A, Volume 21, Supplement 1, 2015, p. S-242.

27. "Stimulation of Human Intra-islet Endothelial Cells Yields Peri-islet Capillaries," G. Loganathan, V.M. Ramakrishnan, N.L. Boyd, J.E. Beare, B. Tweed, L. Kleinert, M.J. Hughes, J.B. Hoying, S.K. Williams, and B.N. Appakalai. International Pancreas and Islet Transplant Association (IPITA)-International Xenotransplantation Association (IXA)- 
Cell Transplant Society (CTS) Joint Congress, Melbourne, Australia (11/2015) ORAL PRESENTATION

28. "Wnt5a Regulates Adipose Stromal Vascular Fraction Self-Assembly," V.M. Ramakrishnan, K.T. Tien, T.R. McKinley, B.R. Bocard, T.M. McCurry, S.K. Williams, J.B. Hoying, and N.L. Boyd. Research!Louisville 2015 Annual Meeting, University of Louisville, Louisville, KY (10/2015)

29. "Quantification of Wnt5a-Mediated Endothelial Network Self-Assembly," T.R. McKinley, V.M. Ramakrishnan, T.M. McCurry, S.K. Williams, J.B. Hoying, and N.L. Boyd. Research!Louisville 2015 Annual Meeting, University of Louisville, Louisville, KY (10/2015)

30. "Stimulation of Intra-islet Endothelial Cells to Form Peri-islet Capillaries (PICs) in Human Islets: Role of Adipose-Derived Stromal Vascular Fraction (SVF) and ECGS solution," G. Loganathan, V.M. Ramakrishnan, N.L. Boyd, J.E. Beare, B. Tweed, L. Kleinert, M.J. Hughes, J.B. Hoying, S.K. Williams, and B.N. Appakalai. Research!Louisville 2015 Annual Meeting, University of Louisville, Louisville, KY (10/2015)

PATENTS: "Cell Systems and Methods for Delivering Disease-Specific Therapies," N.L. Boyd, S.K. Williams, J.B. Hoying, and V.M. Ramakrishnan.

Publication \# WO2014039854 A1

Application \# PCT/US2013/058555

2012

"Device for Circumcision," V.M. Ramakrishnan, R.A. Largo, and J. Vörös.

Filing \# E.P. 11182397.7

2011

INVITED PRESENTATIONS:

1. "Development of an Engineered Implantable Autologous Hepatovascular Unit", UofL Liver Group Seminar, University of Louisville School of Medicine, (02/2015).

2. "Episomal Plasmid Functionally Corrects Monogenic-Deficient Induced Pluripotent Stem Cells," for members of the Institute for Cellular Therapeutics, University of Louisville School of Medicine, (03/2015).

3. "Career Exploration in Medicine and Research", for Georgetown College's Howard Hughes Medical Institutions GC-PALS Program Participants (10/2015). 